\title{
Mixed Ca/Sr salt forms of salicylic acid, tuning structure and aqueous solubility
}

\author{
Pamela Allan, Jean-Baptiste Arlin, Alan R. Kennedy* and Aiden Walls
}

Westchem, Department of Pure \& Applied Chemistry, University of Strathclyde, 295 Cathedral Street, Glasgow G1 1XL, Scotland

Correspondence email: a.r.kennedy@strath.ac.uk

\begin{abstract}
Ten isostructural single-crystal diffraction studies of mixed cation $\mathrm{Ca} / \mathrm{Sr}$ salt forms of the salicylate anion are presented, $\left[\mathrm{Ca}_{(1-x)} \mathrm{Sr}_{x}\left(\mathrm{C}_{7} \mathrm{H}_{5} \mathrm{O}_{3}\right)_{2}\left(\mathrm{OH}_{2}\right)_{2}\right]$, where $x=0,0.041,0.083,0.165,0.306,0.529,0.632,0.789,0.835$ and 1. The structure of an isostructural $\mathrm{Sr} / \mathrm{Ba}$ species $\left[\mathrm{Sr}_{0.729} \mathrm{Ba}_{0.271}\left(\mathrm{C}_{7} \mathrm{H}_{5} \mathrm{O}_{3}\right)_{2}\left(\mathrm{OH}_{2}\right)_{2}\right]$, is also described. The $\mathrm{Ca} / \mathrm{Sr}$ structures form a series where, with increasing $\mathrm{Sr}$ content, the unit cell expands in both the crystallographic a and c directions (by 1.80 and 3.18\% respectively), but contracts slightly in the b direction $(-0.31 \%)$. The largest percentage structural expansion lies parallel to the direction of propagation of the one-dimensional coordination polymer that is the primary structural feature. This structural expansion is thus associated with increased $\mathrm{M}-\mathrm{O}$ distances. Aqueous solubility measurements show that solubility generally increases with increasing $\mathrm{Sr}$ content-Thus tuning the composition of these mixed counterion salt forms leads to sytematic structural changes and allows solubility to be tuned to values between those for the pure $\mathrm{Ca}$ and $\mathrm{Sr}$ species.
\end{abstract}

\section{Comment}

Introduction.

The most common way to alter the material properties of acidic Active Pharmaceutical Ingredients (APIs) is to generate salt forms using s-block metal ions (Stahl, 2008). Commonly the material property of prime interest to manufacturers is aqueous solubility, as this has known links to dissolution rate and hence to bioavailability of APIs. We have previously published a study on comparative structure and solubility trends in series of alkaline earth metal salt forms of pharmaceutically relevant benzoic acid derivatives (Arlin et al., 2011). For salts of the API salicylic acid, this study found a rank order solubility of $\mathrm{Mg}>\mathrm{Ba}>\mathrm{Sr}>\mathrm{Ca}$ and showed that whilst the $\mathrm{Ca}$ and $\mathrm{Sr}$ salts were isostructural and isomorphic, the structures of the $\mathrm{Mg}$ and Ba salts were very different (Arlin et al., 2011; Debuyst et al., 1979). The structures of the Ca and the $\mathrm{Sr}$ salts are both dihydrates with 8-coordinate metal centres and one-dimensional coordination polymer structures that propagate through $\mathrm{Ca}-\mathrm{O}-\mathrm{Ca}$ bridges, where the bridging $\mathrm{O}$ atom is from the carboxylate group. In contrast, the structure of the $\mathrm{Ba}$ salt is a monohydrate two-dimensional coordination polymer and the $\mathrm{Mg}$ salt structure is a discrete tetrahydrate complex of type $\left[\mathrm{Mg}\left(\mathrm{C}_{7} \mathrm{H}_{5} \mathrm{O}_{3}\right)_{2}\left(\mathrm{OH}_{2}\right)_{4}\right]$.

For crystalline systems the isostructuality of the $\mathrm{Ca}$ and $\mathrm{Sr}$ salicylate salt forms should, according to the Hume-Rothery rules, favour the possibility of forming solid solutions with mixed $\mathrm{Ca} / \mathrm{Sr}$ sites (Mizutani, 2011; Hume-Rothery \& Powell, 1935). Structures with such mixed-metal formulations are ubiquitous in inorganic systems (e.g. minerals or alloys, Sekine et al., 2017; Wahlberg et al., 1965; Davidson et al., 2005; Johnson et al., 1970) but are much less studied in molecular or organic salt species. Whilst molecular structures with sites with mutal substitution of s-block metal ions are known both for mixed group 2 metal species (e.g. Trifa et al., 2007) and for mixed group 1 with groups 2 metal species (e.g. Kennedy et al., 1998) there are surprisingly few examples of systematic structural studies of this phenomenon. The most relevant work that we are aware of is a report on the structures of $\mathrm{Ca}$ and $\mathrm{Sr}$ formate, which also gives unit cell data for 3 intermediate mixed $\mathrm{Ca} / \mathrm{Sr}$ forms (Matsui et al., 1980).

Results. 
Eleven samples of salicylate salts were prepared using different initial aqueous $\mathrm{Ca} / \mathrm{Sr}$ ratios. Of these, ten gave singlecrystal samples suitable for accurate structural determination, see Table 1. A 11 structures had similar unit cells to the parent "pure" structures. Where both $\mathrm{Ca}$ and $\mathrm{Sr}$ were present in solution, both metal types were always found incorporated within the structure and sharing one structural site. In all structures, the metal ions occupy a site that is a special position, namely a crystallographic centre of symmetry, Fig. 1. After several trial calculations, all mixed-metal structures were refined with EADP and EXYZ constraints on the metal centres. The composition of the mixed metal site was found by refinement of site occupancy factors, constrained to total 1 . The $\mathrm{Ca} / \mathrm{Sr}$ ratios as measured by diffraction are given in Tables 1 and 13. It can be seen that whilst the single-crystal derived metal ratios do not match the ratios of metals available in the parent solutions, a broad control of crystal composition is possible i.e. the greater the proportion of aqueous $\mathrm{Sr}$ provided, the greater the incorportaion of $\mathrm{Sr}$ into the crystal. Note that all solutions with $>50 \% \mathrm{Ca}$ available gave more $\mathrm{Ca}$ incorporation into the solid than expected simply from the solution composition. The equivalent case for the lower \% $\mathrm{Ca}$ samples is much less clear cut, Table 13.

Attempts were also made to incorporate other group 2 ions into similar structures. No incorporation of Mg was observed, which is perhaps unsurprizing given the large change in ionic radii between $\mathrm{Mg}$ and $\mathrm{Ca}$ and the fundamentally different structure observed for $\mathrm{Mg}$ salicylate as opposed to the $\mathrm{Ca}$ and $\mathrm{Sr}$ structures (Hume-Rothery \& Powell, 1935; Arlin et al., 2011). On both size and structural considerations, the Hume-Rothery rules would suggest that $\mathrm{Ba}$ is a more likely candidate for inclusion. Indeed, a 50:50 Sr/Ba solution did give a mixed metal species, Fig. 2, that is isostructural with the $\mathrm{Ca}$ and $\mathrm{Sr}$ structures described herein. The $\mathrm{Sr} / \mathrm{Ba}$ occupancy refined to 72.9 (4):27.1 (4). The solid thus has much less $\mathrm{Ba}$ present than was available from solution, which perhaps indicates that the larger $\mathrm{Ba}$ ion is not such a good fit to the coordination geometry demands of this structural type.

Unit Cell Changes

As expected, the unit cell volume increases as the amount of $\mathrm{Sr}$ present in the structure increases. Examination of Fig. 3 shows an overall increase in volume of $4.72 \%$. The increase in volume is relatively linear between approximately 10 and $80 \% \mathrm{Sr}$ with small discontinuities at either end of this range. Fig. 4 shows the change in length of the individual a, b and c dimensions. With increasing $\mathrm{Sr}$ content the structure expands in both the a and c directions (by 1.80 and $3.18 \%$ respectively), however the $b$ dimension is relatively invariant and even contracts slightly $(-0.31 \%)$. The $\mathrm{Sr} / \mathrm{Ba}$ structure continues these trends with a larger unit cell volume (1529.04 (11) $\left.\AA^{3}\right)$, expansion of a and c (16.8381 (7) and 7.9433 (3) $\AA$ respectively) and a relatively large contraction of b (to 11.4349 (5) $\AA$ ). This pattern of changes to the unit-cell dimensions with changing metal content is obviously different to the unit cell changes caused by thermal expansion of $\left[\mathrm{Ca}\left(\mathrm{C}_{7} \mathrm{H}_{5} \mathrm{O}_{3}\right)_{2}\left(\mathrm{OH}_{2}\right)_{2}\right]$, Fig. 5, where all axes' dimensions increase with increasing temperature.

Bond Length Considerations.

A simple driver of unit cell expansion is that increasing the amount of the large $\mathrm{Sr}$ ion present leads directly to the observed increase in $\mathrm{M}-\mathrm{O}$ bond distances (where this observed distance is an average of the component $\mathrm{Ca}-\mathrm{O}$ and $\mathrm{Sr}-\mathrm{O}$ distances), Table 14. Of the four structurally independent $\mathrm{M}-\mathrm{O}$ distances, the bond to the neutral water ligand increases more with increasing $\mathrm{Sr}$ content than do the three bonds to formally anionic atoms of carboxylate groups (compare an overall $6.39 \%$ increase for $\mathrm{M} 1-\mathrm{O} 1 \mathrm{~W}$ compared to $4.63,5.40$ and $4.37 \%$ for bonds to $\mathrm{O} 3, \mathrm{O} 3^{\mathrm{ii}}$ and $\mathrm{O} 2{ }^{\mathrm{ii}}$ respectively, $\mathrm{ii}=$ $1-x, 1-y,-z$ ). This fits with the known greater bonding affinity of $\mathrm{Ca}$ for neutral water as compared to $\mathrm{Sr}$ (Kennedy et al., 2009). It also results in $\mathrm{Ca} 1-\mathrm{O} 1 \mathrm{~W}$ being the shortest coordination bond for the $\mathrm{Ca}$ species but $\mathrm{Sr} 1-\mathrm{O} 3$ being the shortest coordination bond for the Sr species, the apparent shortest bond lengths swapping at the 50:50 Ca:Sr point. Similarly, further lengthening of the apparent $\mathrm{M}-\mathrm{O}$ distances occurs in the $\mathrm{Sr} / \mathrm{Ba}$ structure. Again it is $\mathrm{M}-\mathrm{O} 3$ that is shortest with distances of 2.5654 (17), 2.5486 (13), 2.6647 (13) and 2.8113 (13) $\AA$ for $\mathrm{M} 1-\mathrm{O} 1 \mathrm{~W}, \mathrm{M} 1-\mathrm{O} 3, \mathrm{M} 1-\mathrm{O} 3^{\mathrm{ii}}$ and $\mathrm{M} 1-\mathrm{O} 2^{\mathrm{ii}}$ respectively. 
Increasing the average $\mathrm{M}-\mathrm{O}$ distances causes the metal-to-metal seperations along the coordination polymer to expand. As the coordination polymer propagates parallel to the $c$ axis, this explains why it is the c direction that shows the largest percentage expansion. It is harder to explain why the $\mathrm{b}$ dimension does not expand but the a dimension does. Fig. 6 illustrates the packing of the coordination polymers. The metal centres define a rhomboid (of side $10.025 \AA$ for calcium salicylate) and with internal $\mathrm{M}-\mathrm{M}-\mathrm{M}$ angles of approximately 70 and $110^{\circ}$. Those $\mathrm{M}-\mathrm{O}$ vectors that do not lie along the $\mathrm{c}$ direction are diagonaly related to both the $\mathrm{a}$ and $\mathrm{b}$ directions. The hydrogen bonding network follows the same general directions with water-phenol-water interactions linking between coordination chains in both the $\mathrm{a}$ and $\mathrm{b}$ directions, Tables 2 to 12 . The only hydrogen bond interaction that changes systematically with changing metal content is the $\mathrm{O} 1 \mathrm{~W}$ $-\mathrm{H} 2 \mathrm{~W} \cdots \mathrm{O} 2^{\mathrm{ii}}$ bond. This lengthens and becomes more linear with increasing $\mathrm{Sr}$ content. However, this interaction lies along the chain of the coordination polymer and is thus associated with the expansion of the $c$ axis and not the behaviour of a or b. As b is shorter than a, the polymer chains stack closer in the b direction, as measured by $M$ ' $\mathrm{M}$ distances, than in the a direction. A final unique feature of the invariant $\mathrm{b}$ direction is that only in this direction are there close hydrophobic contacts (shortest C“.C 3.2354 (19) $\AA$ ) between aromatic rings. These interactions are highlighted in Fig. 6. These are interdigitated interactions that lie alongside a relatively poorly packed region of the structure. It may be these interdigitated features that buffer the $b$ axis against expansion.

Aqueous Solubility.

The variation of aqueous solubility with increasing $\mathrm{Sr}$ content is illustrated in Fig. 7. Note that here the \% $\mathrm{Sr}$ is calculated by AA analysis of the bulk powders and does not rely on diffraction analysis of single crystals. It is clear that in general solubility increases with increasing $\mathrm{Sr}$ content, with a maximum increase of approximately $50 \%$. Most solubility values lie between those measured for the end points defined by pure calcium salicylate and pure strontium salicylate. Thus it is possible to tune the solubilty of the model API salicylate by using mixed cation formulations. Prior to undertaking this work, we postulated that mixed cation salts may give higher solubility values than their equivalent pure species due to the difficulty of incorporating differently sized "impurity" ions into an energetically minimized lattice. We note that the sample with $82.0 \% \mathrm{Sr}$ content does indeed give a higher solubility than that found for $100 \% \mathrm{Sr}$ but that these points have relatively high associated standard deviations and that the difference is thus not significant. There is thus no evidence for enhanced solubility.

\section{Synthesis and crystallization}

Samples were prepared by dissolving with stirring salicylic acid and the appropriate ratio of calcium carbonate and strontium carbonate in warm water. As an example the Ca:Sr 90:10 sample was prepared from $1.67 \mathrm{~g}$ (12.07 mmol) of salicylic acid, $0.55 \mathrm{~g}(5.49 \mathrm{mmol})$ of $\mathrm{CaCO}_{3}$ and $0.09 \mathrm{~g}(0.61 \mathrm{mmol})$ of $\mathrm{SrCO}_{3}$. Slow evaporation of the solvent gave suitable crystals after 5 to 7 days. These were isolated from the remaining mother liquor by filtration.

Metal ratio of bulk samples was determined by atomic absorption spectrometry as measured by a Thermo Scientific iCE 3300 Series AA spectrometer. A calibration graph was formed using standard solutions containing 1, 2, 2.5, 3, 4 and $5 \mathrm{mg}$ $\mathrm{l}^{-1}$ of Sr ions. Samples of salicylate salts were diluted so as to contain approximately $4 \mathrm{mg} \mathrm{l}^{-1}$ of Sr each.

Aqueous solubility measurements were performed by forming slurries from approximately $1 \mathrm{~g}$ of sample and $8 \mathrm{~cm}^{3}$ of deionized water. The slurries were placed in an incubator at $298 \mathrm{~K}$ and stirred for 10 days. After filtering through 0.2 micron syringe filters, the solutions were diluted to suitable concentration to match a 5 point calibration curve constructed from standard strontium salicylate solutions measured with an Agilent Technologies Cary 60 uv-vis instrument at 295 $\mathrm{cm}^{-1}$. All measurements were performed in duplicate. Solubility results are reported as $\mathrm{mol} / \mathrm{dm}^{3}$ of salicylate ion. The

solids isolated from $100 \% \mathrm{Ca}$ and $100 \% \mathrm{Sr}$ slurries were checked by powder X-ray diffraction which showed the phases isolated to be consistent with the single-crystal structures described herein (Arlin et al., 2011). 
The unit-cell parameters for the variable temperature study are all based on measurements of the same crystal using an Oxford Diffraction Gemini S diffractometer and monochromated $\lambda=1.54148 \AA$ radiation. All cells were calculated using at least 1326 reflections using the program CrysAlis PRO (Agilent, 2014).

\section{Refinement}

For all structures, $\mathrm{H}$ atoms bound to $\mathrm{C}$ atoms were placed in the expected geometric positions and treated in riding modes with $\mathrm{C}-\mathrm{H}=0.95$ and $\mathrm{U}(\mathrm{H})_{\mathrm{iso}}=1.2 \mathrm{U}(\mathrm{C})_{\mathrm{eq}} . \mathrm{H}$ atoms bound to $\mathrm{O}$ atoms were located by difference synthesis and refined isotropically. For the structures with $100 \% \mathrm{Ca}$ and $100 \% \mathrm{Sr}$ it was found neccessary to restrain $\mathrm{O}-\mathrm{H}$ distances to 0.85 (2) Å.

\section{Table 1}

Experimental details

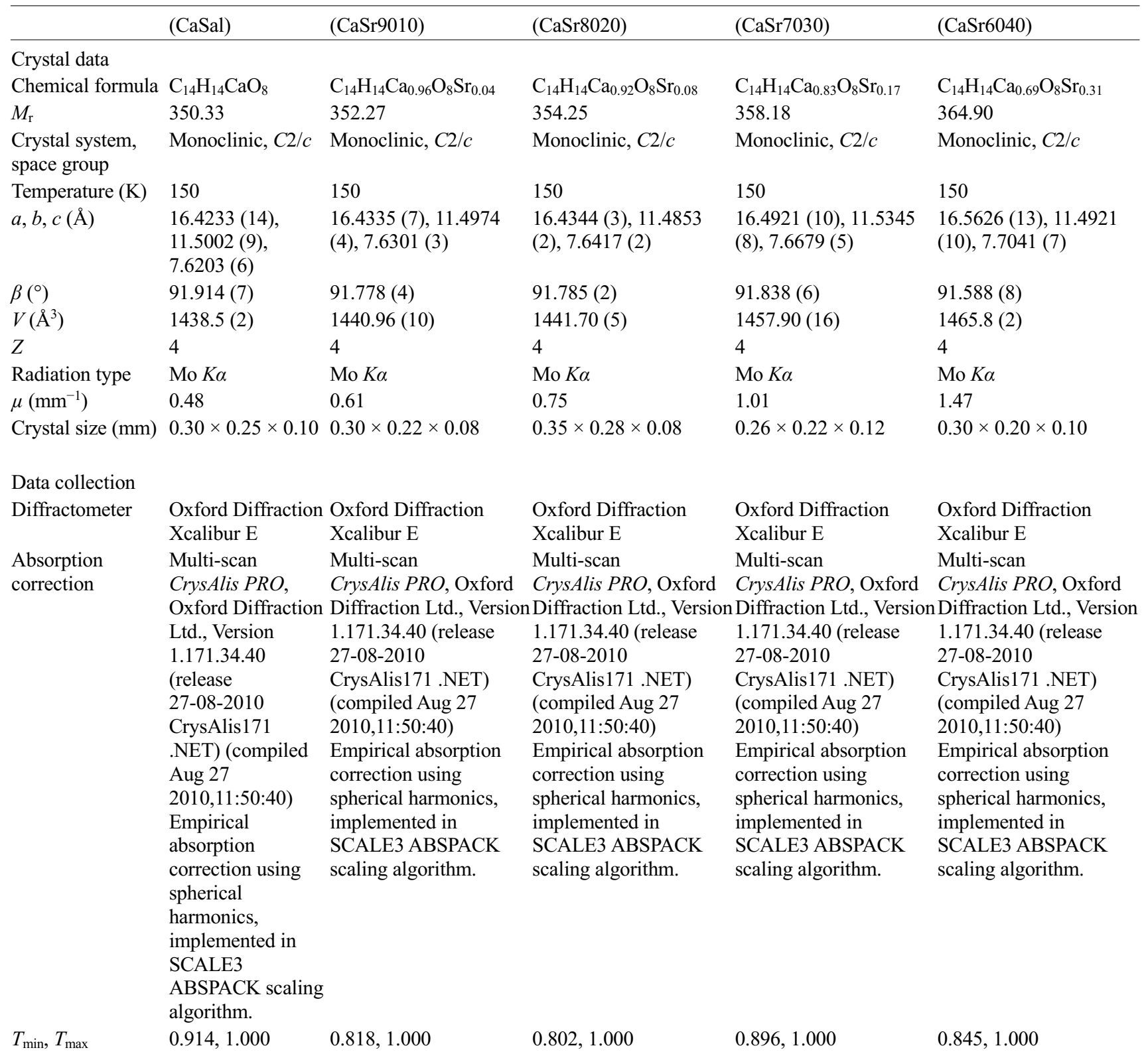




\begin{tabular}{|c|c|c|c|c|c|}
\hline $\begin{array}{l}\text { No. of measured, } \\
\text { independent and } \\
\text { observed }[I> \\
2 \sigma(I)] \text { reflections }\end{array}$ & $6630,1638,1489$ & $16338,1640,1582$ & $16552,1642,1568$ & $3241,1659,1494$ & $9041,1656,1576$ \\
\hline$R_{\text {int }}$ & 0.027 & 0.036 & 0.030 & 0.032 & 0.035 \\
\hline$(\sin \theta / \lambda)_{\max }\left(\AA^{-1}\right)$ & 0.649 & 0.649 & 0.650 & 0.650 & 0.650 \\
\hline \multicolumn{6}{|l|}{ Refinement } \\
\hline $\begin{array}{l}R\left[F^{2}>2 \sigma\left(F^{2}\right)\right], \\
w R\left(F^{2}\right), S\end{array}$ & $0.027,0.068,1.08$ & $0.021,0.055,1.07$ & $0.021,0.053,1.08$ & $0.036,0.093,1.09$ & $0.024,0.056,1.11$ \\
\hline No. of reflections & 1638 & 1640 & 1642 & 1659 & 1656 \\
\hline No. of parameters & 117 & 118 & 118 & 118 & 118 \\
\hline No. of restraints & 2 & 0 & 0 & 0 & 0 \\
\hline $\mathrm{H}$-atom treatment & $\begin{array}{l}\mathrm{H} \text { atoms treated by } \\
\text { a mixture of } \\
\text { independent and } \\
\text { constrained } \\
\text { refinement }\end{array}$ & $\begin{array}{l}\mathrm{H} \text { atoms treated by a } \\
\text { mixture of independent } \\
\text { and constrained } \\
\text { refinement }\end{array}$ & $\begin{array}{l}\mathrm{H} \text { atoms treated by a } \\
\text { mixture of independent } \\
\text { and constrained } \\
\text { refinement }\end{array}$ & $\begin{array}{l}\mathrm{H} \text { atoms treated by a } \\
\text { mixture of independent } \\
\text { and constrained } \\
\text { refinement }\end{array}$ & $\begin{array}{l}\mathrm{H} \text { atoms treated by a } \\
\text { mixture of independent } \\
\text { and constrained } \\
\text { refinement }\end{array}$ \\
\hline $\begin{array}{l}\Delta \rho_{\max }, \Delta \rho_{\min }(\mathrm{e} \\
\left.\AA^{-3}\right)\end{array}$ & $0.34,-0.20$ & $0.29,-0.21$ & $0.31,-0.23$ & $0.37,-0.38$ & $0.28,-0.20$ \\
\hline
\end{tabular}

\begin{tabular}{|c|c|c|c|c|c|}
\hline & $(\mathrm{CaSr} 5050)$ & $(\mathrm{CaSr} 3070)$ & $(\mathrm{CaSr} 2080)$ & (CaSr1090) & $(\mathrm{Sr} 100)$ \\
\hline \multicolumn{6}{|l|}{ Crystal data } \\
\hline Chemical formula & $\mathrm{C}_{14} \mathrm{H}_{14} \mathrm{Ca}_{0.47} \mathrm{O}_{8} \mathrm{Sr}_{0.53}$ & $\mathrm{C}_{14} \mathrm{H}_{14} \mathrm{Ca}_{0.37} \mathrm{O}_{8} \mathrm{Sr}_{0.63}$ & $\mathrm{C}_{14} \mathrm{H}_{14} \mathrm{Ca}_{0.21} \mathrm{O}_{8} \mathrm{Sr}_{0.79}$ & $\mathrm{C}_{14} \mathrm{H}_{14} \mathrm{Ca}_{0.17} \mathrm{O}_{8} \mathrm{Sr}_{0.83}$ & $\mathrm{C}_{14} \mathrm{H}_{14} \mathrm{O}_{8} \mathrm{Sr}$ \\
\hline$M_{\mathrm{r}}$ & 375.48 & 380.38 & 387.85 & 390.00 & 397.87 \\
\hline $\begin{array}{l}\text { Crystal system, } \\
\text { space group }\end{array}$ & Monoclinic, $C 2 / c$ & Monoclinic, $C 2 / c$ & Monoclinic, $C 2 / c$ & Monoclinic, $C 2 / c$ & Monoclinic, $C 2 / c$ \\
\hline Temperature $(\mathrm{K})$ & 150 & 150 & 150 & 150 & 150 \\
\hline$a, b, c(\AA)$ & $\begin{array}{l}16.5994(10), 11.4832 \\
(6), 7.7650(5)\end{array}$ & $\begin{array}{l}16.6319(6), 11.4995 \\
(4), 7.7729(3)\end{array}$ & $\begin{array}{l}16.6650(11), 11.4816 \\
(7), 7.8105(5)\end{array}$ & $\begin{array}{l}16.6693(9), 11.4865 \\
(7), 7.8446(4)\end{array}$ & $\begin{array}{l}16.7182(6), \\
11.4644(4), 7.8627 \\
(3)\end{array}$ \\
\hline$\beta\left(^{\circ}\right)$ & $91.555(5)$ & $91.599(4)$ & $91.576(6)$ & $91.510(5)$ & $91.660(3)$ \\
\hline$V\left(\AA^{3}\right)$ & $1479.57(15)$ & $1486.05(9)$ & $1493.90(16)$ & $1501.50(14)$ & $1506.37(9)$ \\
\hline$Z$ & 4 & 4 & 4 & 4 & 4 \\
\hline Radiation type & Mo $K \alpha$ & Mo $K \alpha$ & Mo $K \alpha$ & Mo $K \alpha$ & $\mathrm{Cu} K \alpha$ \\
\hline$\mu\left(\mathrm{mm}^{-1}\right)$ & 2.17 & 2.49 & 2.98 & 3.11 & 5.36 \\
\hline Crystal size (mm) & $0.30 \times 0.20 \times 0.10$ & $0.30 \times 0.25 \times 0.12$ & $0.26 \times 0.25 \times 0.15$ & $0.30 \times 0.15 \times 0.06$ & $0.6 \times 0.5 \times 0.2$ \\
\hline \multicolumn{6}{|l|}{ Data collection } \\
\hline Diffractometer & $\begin{array}{l}\text { Oxford Diffraction } \\
\text { Xcalibur E }\end{array}$ & $\begin{array}{l}\text { Oxford Diffraction } \\
\text { Xcalibur E }\end{array}$ & $\begin{array}{l}\text { Oxford Diffraction } \\
\text { Xcalibur E }\end{array}$ & $\begin{array}{l}\text { Oxford Diffraction } \\
\text { Xcalibur E }\end{array}$ & $\begin{array}{l}\text { Oxford Diffraction } \\
\text { Gemini S }\end{array}$ \\
\hline
\end{tabular}




\begin{tabular}{|c|c|c|c|c|c|}
\hline $\begin{array}{l}\text { Absorption } \\
\text { correction }\end{array}$ & $\begin{array}{l}\text { Multi-scan } \\
\text { CrysAlis PRO, Oxford } \\
\text { Diffraction Ltd., Version }\end{array}$ & $\begin{array}{l}\text { Multi-scan } \\
\text { CrysAlis PRO, Oxford } \\
\text { ADiffraction Ltd., Version }\end{array}$ & $\begin{array}{l}\text { Multi-scan } \\
\text { CrysAlis PRO, Oxford } \\
\text { Diffraction Ltd., Versior }\end{array}$ & $\begin{array}{l}\text { Multi-scan } \\
\text { CrysAlis PRO, Oxford } \\
\text { aDiffraction Ltd., Version }\end{array}$ & $\begin{array}{l}\text { Analytical } \\
\text { CrysAlis PRO, } \\
\text { Agilent }\end{array}$ \\
\hline & $\begin{array}{l}\text { 1.171.34.40 (release } \\
\text { 27-08-2010 } \\
\text { CrysAlis171 .NET) } \\
\text { (compiled Aug } 27 \\
\text { 2010,11:50:40) } \\
\text { Empirical absorption } \\
\text { correction using } \\
\text { spherical harmonics, } \\
\text { implemented in } \\
\text { SCALE3 ABSPACK } \\
\text { scaling algorithm. }\end{array}$ & $\begin{array}{l}\text { 1.171.34.40 (release } \\
\text { 27-08-2010 } \\
\text { CrysAlis171 .NET) } \\
\text { (compiled Aug } 27 \\
\text { 2010,11:50:40) } \\
\text { Empirical absorption } \\
\text { correction using } \\
\text { spherical harmonics, } \\
\text { implemented in } \\
\text { SCALE3 ABSPACK } \\
\text { scaling algorithm. }\end{array}$ & $\begin{array}{l}\text { 1.171.34.40 (release } \\
\text { 27-08-2010 } \\
\text { CrysAlis171 .NET) } \\
\text { (compiled Aug } 27 \\
\text { 2010,11:50:40) } \\
\text { Empirical absorption } \\
\text { correction using } \\
\text { spherical harmonics, } \\
\text { implemented in } \\
\text { SCALE3 ABSPACK } \\
\text { scaling algorithm. }\end{array}$ & $\begin{array}{l}\text { 1.171.34.40 (release } \\
\text { 27-08-2010 } \\
\text { CrysAlis171 .NET) } \\
\text { (compiled Aug } 27 \\
\text { 2010,11:50:40) } \\
\text { Empirical absorption } \\
\text { correction using } \\
\text { spherical harmonics, } \\
\text { implemented in } \\
\text { SCALE3 ABSPACK } \\
\text { scaling algorithm. }\end{array}$ & $\begin{array}{l}\text { Technologies, } \\
\text { Version } \\
\text { 1.171.37.35 } \\
\text { (release } \\
\text { 13-08-2014 } \\
\text { CrysAlis171 .NET) } \\
\text { (compiled Aug 13 } \\
\text { 2014,18:06:01) } \\
\text { Analytical numeric } \\
\text { absorption } \\
\text { correction using a } \\
\text { multifaceted } \\
\text { crystal model } \\
\text { based on } \\
\text { expressions } \\
\text { derived by R.C. } \\
\text { Clark \& J.S. Reid. } \\
\text { (Clark, R. C. \& } \\
\text { Reid, J. S. (1995). } \\
\text { Acta Cryst. A51, } \\
\text { 887-897) Empirical } \\
\text { absorption } \\
\text { correction using } \\
\text { spherical } \\
\text { harmonics, } \\
\text { implemented in } \\
\text { SCALE3 } \\
\text { ABSPACK scaling } \\
\text { algorithm. }\end{array}$ \\
\hline$T_{\min }, T_{\max }$ & $0.847,1.000$ & $0.735,1.000$ & $0.896,1.000$ & $0.462,1.000$ & $0.120,0.461$ \\
\hline $\begin{array}{l}\text { No. of measured, } \\
\text { independent and } \\
\text { observed }[I> \\
2 \sigma(I)] \text { reflections }\end{array}$ & $8207,1691,1546$ & $16351,1704,1622$ & $6802,1716,1594$ & $8538,1720,1589$ & $2454,1272,1259$ \\
\hline$R_{\text {int }}$ & 0.046 & 0.036 & 0.041 & 0.044 & 0.016 \\
\hline$(\sin \theta / \lambda)_{\max }\left(\AA^{-1}\right)$ & 0.650 & 0.650 & 0.649 & 0.650 & 0.591 \\
\hline $\begin{array}{l}\text { Refinement } \\
R\left[F^{2}>2 \sigma\left(F^{2}\right)\right], \\
w R\left(F^{2}\right), S\end{array}$ & $0.027,0.058,1.12$ & $0.021,0.052,1.06$ & $0.024,0.054,1.06$ & $0.027,0.065,1.08$ & $0.027,0.073,1.17$ \\
\hline No. of reflections & 1691 & 1704 & 1716 & 1720 & 1272 \\
\hline No. of parameters & 118 & 118 & 118 & 118 & 118 \\
\hline No. of restraints & 0 & 0 & 0 & 0 & 3 \\
\hline $\mathrm{H}$-atom treatment & $\begin{array}{l}\mathrm{H} \text { atoms treated by a } \\
\text { mixture of independent } \\
\text { and constrained } \\
\text { refinement }\end{array}$ & $\begin{array}{l}\mathrm{H} \text { atoms treated by a } \\
\text { mixture of independent } \\
\text { and constrained } \\
\text { refinement }\end{array}$ & $\begin{array}{l}\mathrm{H} \text { atoms treated by a } \\
\text { mixture of independent } \\
\text { and constrained } \\
\text { refinement }\end{array}$ & $\begin{array}{l}\mathrm{H} \text { atoms treated by a } \\
\text { mixture of independent } \\
\text { and constrained } \\
\text { refinement }\end{array}$ & $\begin{array}{l}\mathrm{H} \text { atoms treated by } \\
\text { a mixture of } \\
\text { independent and } \\
\text { constrained } \\
\text { refinement }\end{array}$ \\
\hline $\begin{array}{l}\Delta \rho_{\max }, \Delta \rho_{\min }(\mathrm{e} \\
\left.\AA^{-3}\right)\end{array}$ & $0.25,-0.28$ & $0.49,-0.25$ & $0.31,-0.32$ & $0.70,-0.48$ & $0.68,-0.49$ \\
\hline
\end{tabular}

(SrBa) 


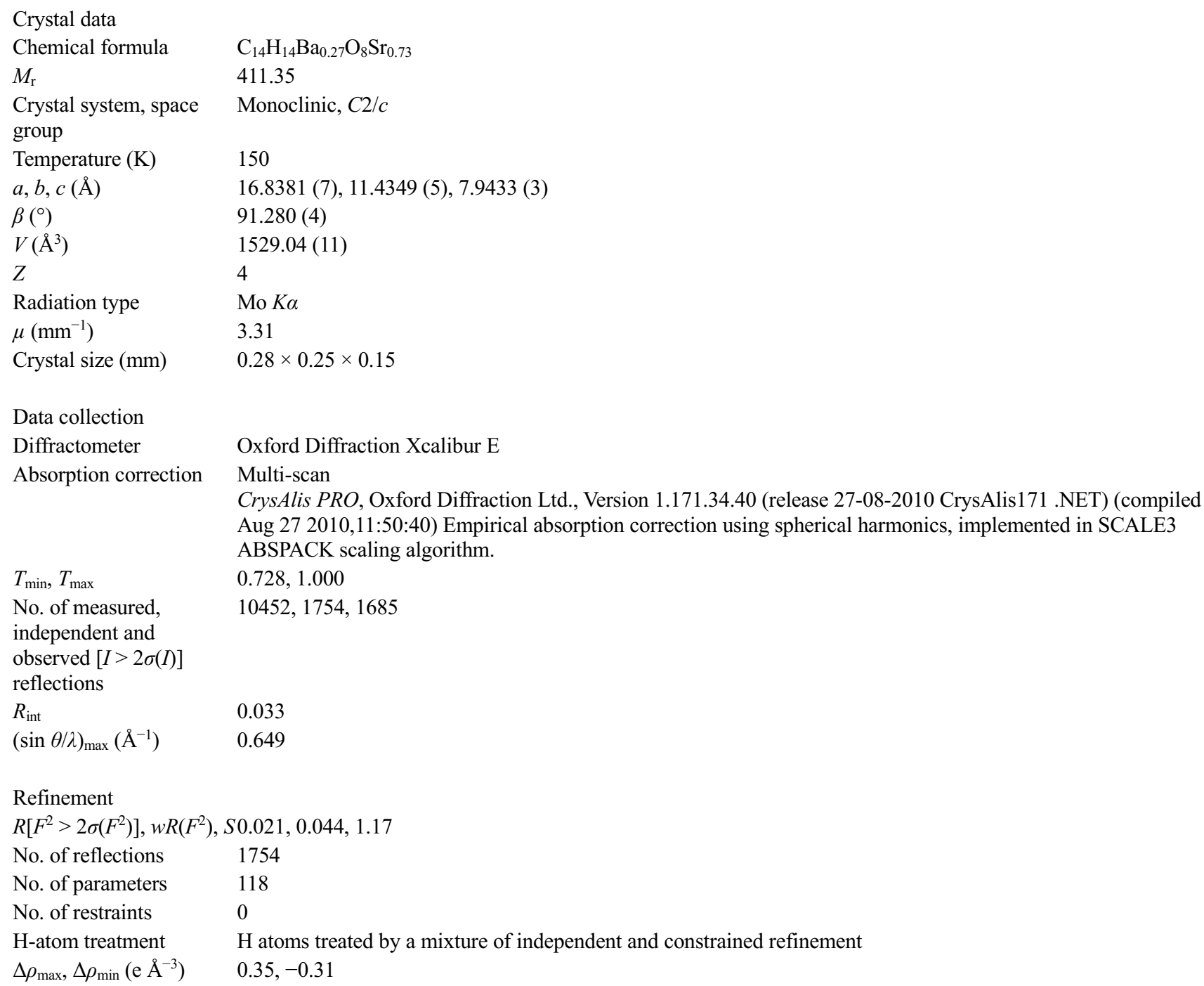

Data collection Diffractometer Absorption correction

Oxford Diffraction Xcalibur E

Multi-scan

CrysAlis PRO, Oxford Diffraction Ltd., Version 1.171.34.40 (release 27-08-2010 CrysAlis171 .NET) (compiled Aug 27 2010,11:50:40) Empirical absorption correction using spherical harmonics, implemented in SCALE3 ABSPACK scaling algorithm.

$T_{\min }, T_{\max }$ $0.728,1.000$

No. of measured, independent and $10452,1754,1685$

observed $[I>2 \sigma(I)]$

reflections

$\begin{array}{ll}R_{\text {int }} & 0.033 \\ (\sin \theta / \lambda)_{\max }\left(\AA^{-1}\right) & 0.649\end{array}$

Refinement

$R\left[F^{2}>2 \sigma\left(F^{2}\right)\right], w R\left(F^{2}\right), S 0.021,0.044,1.17$

No. of reflections 1754

No. of parameters $\quad 118$

No. of restraints $\quad 0$

$\mathrm{H}$-atom treatment $\quad \mathrm{H}$ atoms treated by a mixture of independent and constrained refinement

$\Delta \rho_{\max }, \Delta \rho_{\min }\left(\mathrm{e} \AA^{-3}\right) \quad 0.35,-0.31$

Computer programs: CrysAlis PRO (Agilent, 2014), CrysAlis PRO, SIR92 (Altomare et al., 1994), SHELXL2014 (Sheldrick, 2015), Mercury (Macrae, 2008), SHELXL2014.

\section{Table 2}

Hydrogen-bond geometry $\left(\AA,{ }^{\circ}\right)$

\begin{tabular}{lllll}
\hline$D-\mathrm{H} \cdots A$ & $D-\mathrm{H}$ & $\mathrm{H} \cdots A$ & $D \cdots A$ & $D-\mathrm{H} \cdots A$ \\
\hline $\mathrm{O} 1-\mathrm{H} 1 O \cdots \mathrm{O} 2$ & $0.81(2)$ & $1.90(2)$ & $2.6256(13)$ & $148(2)$ \\
$\mathrm{O} 1 W-\mathrm{H} 1 W \cdots \mathrm{O} 1^{\mathrm{i}}$ & $0.82(2)$ & $2.03(2)$ & $2.8391(14)$ & $169(2)$ \\
$\mathrm{O} 1 W-\mathrm{H} 2 W \cdots \mathrm{O} 2^{\mathrm{ii}}$ & $0.84(2)$ & $2.05(2)$ & $2.8795(14)$ & $169(2)$ \\
\hline
\end{tabular}

Symmetry codes: (i) $-x+1 / 2,-y+1 / 2,-z$; (ii) $x, y, z-1$. 
Table 3

Hydrogen-bond geometry $\left(\AA,{ }^{\circ}\right)$

\begin{tabular}{lllll}
\hline$D-\mathrm{H} \cdots A$ & $D-\mathrm{H}$ & $\mathrm{H} \cdots A$ & $D \cdots A$ & $D-\mathrm{H} \cdots A$ \\
\hline $\mathrm{O} 1-\mathrm{H} 1 O \cdots \mathrm{O} 2$ & $0.872(18)$ & $1.842(19)$ & $2.6204(10)$ & $147.6(16)$ \\
$\mathrm{O} 1 W-\mathrm{H} 1 W \cdots \mathrm{O}^{\mathrm{i}}$ & $0.864(19)$ & $1.981(19)$ & $2.8361(11)$ & $169.8(16)$ \\
$\mathrm{O} 1 W-\mathrm{H} 2 W \cdots \mathrm{O}^{\mathrm{ii}}$ & $0.853(18)$ & $2.043(18)$ & $2.8820(11)$ & $167.6(15)$ \\
\hline
\end{tabular}

Symmetry codes: (i) $-x+1 / 2,-y+1 / 2,-z$; (ii) $x, y, z-1$.

\section{Table 4}

Hydrogen-bond geometry $\left(\AA,{ }^{\circ}\right)$

\begin{tabular}{lllll}
\hline$D-\mathrm{H} \cdots A$ & $D-\mathrm{H}$ & $\mathrm{H} \cdots A$ & $D \cdots A$ & $D-\mathrm{H} \cdots A$ \\
\hline $\mathrm{O} 1-\mathrm{H} 1 O \cdots \mathrm{O} 2$ & $0.865(18)$ & $1.857(18)$ & $2.6165(11)$ & $145.5(16)$ \\
$\mathrm{O} 1 W-\mathrm{H} 1 W \cdots \mathrm{O} 1^{\mathrm{i}}$ & $0.85(2)$ & $2.00(2)$ & $2.8350(11)$ & $169.7(17)$ \\
$\mathrm{O} 1 W-\mathrm{H} 2 W \cdots \mathrm{O} 2^{\mathrm{ii}}$ & $0.836(19)$ & $2.06(2)$ & $2.8849(12)$ & $166.8(17)$ \\
\hline
\end{tabular}

Symmetry codes: (i) $-x+1 / 2,-y+1 / 2,-z$; (ii) $x, y, z-1$.

\section{Table 5}

Hydrogen-bond geometry $\left(\AA,{ }^{\circ}\right)$

\begin{tabular}{lllll}
\hline$D-\mathrm{H} \cdots A$ & $D-\mathrm{H}$ & $\mathrm{H} \cdots A$ & $D \cdots A$ & $D-\mathrm{H} \cdots A$ \\
\hline $\mathrm{O} 1-\mathrm{H} 1 O \cdots \mathrm{O} 2$ & $0.87(3)$ & $1.86(3)$ & $2.6216(18)$ & $145(3)$ \\
$\mathrm{O} 1 W-\mathrm{H} 1 W \cdots \mathrm{O} 1^{\mathrm{i}}$ & $0.87(3)$ & $1.99(3)$ & $2.8409(19)$ & $166(3)$ \\
$\mathrm{O} 1 W-\mathrm{H} 2 W \cdots \mathrm{O} 2^{\mathrm{ii}}$ & $0.86(3)$ & $2.06(3)$ & $2.891(2)$ & $161(3)$ \\
\hline
\end{tabular}

Symmetry codes: (i) $-x+1 / 2,-y+1 / 2,-z$; (ii) $x, y, z-1$.

\section{Table 6}

Hydrogen-bond geometry $\left(\AA,{ }^{\circ}\right)$

\begin{tabular}{lllll}
\hline$D-\mathrm{H} \cdots A$ & $D-\mathrm{H}$ & $\mathrm{H} \cdots A$ & $D \cdots A$ & $D-\mathrm{H} \cdots A$ \\
\hline $\mathrm{O} 1-\mathrm{H} 1 O \cdots \mathrm{O} 2$ & $0.87(2)$ & $1.83(2)$ & $2.6174(14)$ & $148(2)$ \\
$\mathrm{O} 1 W-\mathrm{H} 1 W \cdots \mathrm{O} 1^{\mathrm{i}}$ & $0.89(3)$ & $1.95(3)$ & $2.8372(15)$ & $170(2)$ \\
$\mathrm{O} 1 W-\mathrm{H} 2 W \cdots \mathrm{O} 2^{\mathrm{ii}}$ & $0.89(2)$ & $2.02(2)$ & $2.8984(16)$ & $168.7(19)$ \\
\hline
\end{tabular}

Symmetry codes: (i) $-x+1 / 2,-y+1 / 2,-z$; (ii) $x, y, z-1$.

\section{Table 7}

Hydrogen-bond geometry $\left(\AA,{ }^{\circ}\right)$

\begin{tabular}{lllll}
\hline$D-\mathrm{H} \cdots A$ & $D-\mathrm{H}$ & $\mathrm{H} \cdots A$ & $D \cdots A$ & $D-\mathrm{H} \cdots A$ \\
\hline $\mathrm{O} 1-\mathrm{H} 1 O \cdots \mathrm{O} 2$ & $0.90(3)$ & $1.81(3)$ & $2.6075(17)$ & $147(2)$ \\
$\mathrm{O} 1 W-\mathrm{H} 1 W \cdots \mathrm{O} 1^{\mathrm{i}}$ & $0.86(3)$ & $1.97(3)$ & $2.8276(19)$ & $171(2)$ \\
$\mathrm{O} 1 W-\mathrm{H} 2 W \cdots \mathrm{O} 2^{\mathrm{ii}}$ & $0.90(3)$ & $2.02(3)$ & $2.906(2)$ & $169(2)$ \\
\hline
\end{tabular}

Symmetry codes: (i) $-x+1 / 2,-y+1 / 2,-z$; (ii) $x, y, z-1$. 


\section{Table 8}

Hydrogen-bond geometry $\left(\AA,{ }^{\circ}\right)$

\begin{tabular}{lllll}
\hline$D-\mathrm{H} \cdots A$ & $D-\mathrm{H}$ & $\mathrm{H} \cdots A$ & $D \cdots A$ & $D-\mathrm{H} \cdots A$ \\
\hline $\mathrm{O} 1-\mathrm{H} 1 O \cdots \mathrm{O} 2$ & $0.90(3)$ & $1.81(3)$ & $2.6096(15)$ & $147(2)$ \\
$\mathrm{O} 1 W-\mathrm{H} 1 W \cdots \mathrm{O} 1^{\mathrm{i}}$ & $0.84(3)$ & $1.99(3)$ & $2.8255(16)$ & $175(3)$ \\
$\mathrm{O} 1 W-\mathrm{H} 2 W \cdots 2^{\mathrm{ii}}$ & $0.85(2)$ & $2.06(2)$ & $2.9074(17)$ & $173(2)$ \\
\hline
\end{tabular}

Symmetry codes: (i) $-x+1 / 2,-y+1 / 2,-z$; (ii) $x, y, z-1$.

\section{Table 9}

Hydrogen-bond geometry $\left(\AA,{ }^{\circ}\right)$

\begin{tabular}{lllll}
\hline$D-\mathrm{H} \cdots A$ & $D-\mathrm{H}$ & $\mathrm{H} \cdots A$ & $D \cdots A$ & $D-\mathrm{H} \cdots A$ \\
\hline $\mathrm{O} 1-\mathrm{H} 1 O \cdots \mathrm{O} 2$ & $0.89(3)$ & $1.84(3)$ & $2.6031(17)$ & $142(2)$ \\
$\mathrm{O} 1 W-\mathrm{H} 1 W \cdots \mathrm{O} 1^{\mathrm{i}}$ & $0.88(3)$ & $1.94(3)$ & $2.8244(18)$ & $173(2)$ \\
$\mathrm{O} 1 W-\mathrm{H} 2 W \cdots \mathrm{O} 2^{\mathrm{ii}}$ & $0.85(3)$ & $2.06(3)$ & $2.911(2)$ & $178(2)$ \\
\hline
\end{tabular}

Symmetry codes: (i) $-x+1 / 2,-y+1 / 2,-z$; (ii) $x, y, z-1$.

\section{Table 10}

Hydrogen-bond geometry $\left(\AA,{ }^{\circ}\right)$

\begin{tabular}{lllll}
\hline$D-\mathrm{H} \cdots A$ & $D-\mathrm{H}$ & $\mathrm{H} \cdots A$ & $D \cdots A$ & $D-\mathrm{H} \cdots A$ \\
\hline $\mathrm{O} 1-\mathrm{H} 1 O \cdots \mathrm{O} 2$ & $0.90(3)$ & $1.80(3)$ & $2.597(2)$ & $147(3)$ \\
$\mathrm{O} 1 W-\mathrm{H} 1 W \cdots \mathrm{O} 1^{\mathrm{i}}$ & $0.81(3)$ & $2.03(3)$ & $2.827(2)$ & $173(3)$ \\
$\mathrm{O} 1 W-\mathrm{H} 2 W \cdots \mathrm{O} 2^{\mathrm{ii}}$ & $0.92(3)$ & $2.01(3)$ & $2.921(2)$ & $173(3)$ \\
\hline
\end{tabular}

Symmetry codes: (i) $-x+1 / 2,-y+1 / 2,-z$; (ii) $x, y, z-1$.

\section{Table 11}

Hydrogen-bond geometry $\left(\AA,{ }^{\circ}\right)$

\begin{tabular}{lllll}
\hline$D-\mathrm{H} \cdots A$ & $D-\mathrm{H}$ & $\mathrm{H} \cdots A$ & $D \cdots A$ & $D-\mathrm{H} \cdots A$ \\
\hline $\mathrm{O} 1-\mathrm{H} 1 O \cdots \mathrm{O} 2$ & $0.83(2)$ & $1.89(3)$ & $2.604(2)$ & $145(4)$ \\
$\mathrm{O} 1 W-\mathrm{H} 1 W \cdots \mathrm{O} 1^{\mathrm{i}}$ & $0.82(2)$ & $2.01(2)$ & $2.823(3)$ & $172(4)$ \\
$\mathrm{O} 1 W-\mathrm{H} 2 W \cdots \mathrm{O} 2^{\mathrm{ii}}$ & $0.84(2)$ & $2.08(2)$ & $2.925(3)$ & $178(4)$ \\
\hline
\end{tabular}

Symmetry codes: (i) $-x+1 / 2,-y+1 / 2,-z$; (ii) $x, y, z-1$.

Table 12

Hydrogen-bond geometry $\left(\AA,{ }^{\circ}\right)$

\begin{tabular}{lllll}
\hline$D-\mathrm{H} \cdots A$ & $D-\mathrm{H}$ & $\mathrm{H} \cdots A$ & $D \cdots A$ & $D-\mathrm{H} \cdots A$ \\
\hline $\mathrm{O} 1-\mathrm{H} 7 \cdots \mathrm{O} 2$ & $0.89(3)$ & $1.80(3)$ & $2.5938(18)$ & $148(3)$ \\
$\mathrm{O} 1 W-\mathrm{H} 1 W^{\cdots} \cdots \mathrm{O} 1^{\mathrm{i}}$ & $0.82(3)$ & $2.00(3)$ & $2.820(2)$ & $172(3)$ \\
$\mathrm{O} 1 W-\mathrm{H} 2 W \cdots \mathrm{O} 2^{\mathrm{ii}}$ & $0.86(3)$ & $2.08(3)$ & $2.923(2)$ & $169(3)$ \\
\hline
\end{tabular}

Symmetry codes: (i) $-x+1 / 2,-y+1 / 2,-z$; (ii) $x, y, z-1$. 


\section{Table 13}

Solubility data and $\mathrm{Ca} / \mathrm{Sr}$ ratios. The ratios are given as, that available from parent solution, that determined from singlecrystal refinement and that determined from AA for bulk sample.

\begin{tabular}{llll}
\hline Sr \% in Solution & Sr \% single-crystal & Sr \% bulk AA & \multicolumn{1}{c}{ Solubility mol/dm ${ }^{3}$} \\
0 & 0 & 0 & $0.185(4)$ \\
9.9 & $4.1(2)$ & 7.01 & $0.195(2)$ \\
19.9 & $8.3(2)$ & 16.04 & $0.201(2)$ \\
30.2 & $16.5(3)$ & 23.67 & $0.209(2)$ \\
39.7 & $30.6(3)$ & 36.13 & $0.235(10)$ \\
49.5 & $52.9(3)$ & 51.88 & $0.231(4)$ \\
60.3 & & 56.67 & $0.242(8)$ \\
69.8 & $63.2(4)$ & 75.59 & $0.235(6)$ \\
80.7 & $78.9(4)$ & 82.01 & $0.279(20)$ \\
88.8 & $83.5(5)$ & 90.28 & $0.257(8)$ \\
100 & 100 & 100 & $0.257(10)$
\end{tabular}

\section{Table 14}

Variation in apparent $M-\mathrm{O}$ bond lengths $(\AA)$ for different $\mathrm{Ca}$ :Sr ratios. ii $=1-x, 1-y,-z$.

\begin{tabular}{lllll}
\hline Sr \% from single-crystal & M-O1W & M-O3 & M-O3 & M-O2 $^{\mathrm{ii}}$ \\
0 & $2.3761(10)$ & $2.4005(9)$ & $2.4781(9)$ & $2.6333(9)^{\prime}$ \\
$4.1(2)$ & $2.3814(8)$ & $2.4027(7)$ & $2.4910(7)$ & $2.6458(7)$ \\
$8.3(2)$ & $2.3830(9)$ & $2.4062(8)$ & $2.4968(8)$ & $2.6506(8)$ \\
$16.5(3)$ & $2.3968(15)$ & $2.4224(13)$ & $2.5166(14)$ & $2.6739(13)$ \\
$30.6(3)$ & $2.4211(11)$ & $2.4324(10)$ & $2.5336(10)$ & $2.6907(10)$ \\
$52.9(3)$ & $2.4608(15)$ & $2.4596(13)$ & $2.5621(13)$ & $2.7143(12)$ \\
$63.2(4)$ & $2.4777(12)$ & $2.4686(10)$ & $2.5749(10)$ & $2.7260(10)$ \\
$78.9(4)$ & $2.5020(14)$ & $2.4849(12)$ & $2.5902(12)$ & $2.7368(12)$ \\
$83.5(5)$ & $2.5091(17)$ & $2.4953(13)$ & $2.5990(14)$ & $2.7400(15)$ \\
100 & $2.528(2)$ & $2.5116(17)$ & $2.6120(17)$ & $2.7484(17)$
\end{tabular}

\section{Acknowledgements}

\section{Funding information}

\section{References}

Agilent (2014). CrysAlis PRO. Agilent Technologies Ltd., Yarnton, Oxfordshire, England.

Altomare, A., Cascarano, G., Giacovazzo, C., Guagliardi, A., Burla, M. C., Polidori, G. \& Camalli, M. (1994). J. Appl. Cryst. 27, 435.

Arlin, J.-B., Florence, A. J., Johnston, A., Kennedy, A. R., Miller, G. J. \& Patterson, K. (2011). Cryst. Growth Des. 11, $1318-1327$.

Davidson, C. M., Gibson, M. D., Hamilton, E., MacGillivray, B. H., Reglinski, J. \& Rezabal, E. (2005). Chemosphere 58, $793-798$. 
Debuyst, R., Dejehet, F., Dekandelaer, M.-C., Declercq, J. P. \& van Meerssche, M. (1979). Phys.-Chim. Biol. 76, $1117-$ 1124.

Farrugia, L. J. (2012). J. Appl. Cryst. 45, 849-854.

Hume-Rothery, W. \& Powell, H. M. (1935). Z. Krist. 91, 23-47.

Johnson, A. R., Armstrong, W. D. \& Singer, L. (1970). Calc. Tiss. Res. 6, 103-112.

Kennedy, A. R., Andrikopoulos, P. C., Arlin, J.-B., Armstrong, D. R., Duxbury, N., Graham, D. V. \& Kirkhouse, J. B. A. (2009). Chem. Eur. J. 15, 9494-9504.

Kennedy, A. R., Mulvey, R. E. \& Rowlings, R. B. (1998). Angew. Chem. Int. Ed. 37, 3180-3183.

Macrae, C. F., Bruno, I. J., Chisholm, J. A., Edgington, P. R., McCabe, P., Pidcock, E., Rodriguez Monge, L., Taylor, R., van de Streek, J. \& Wood, P. A. (2008). J. Appl. Cryst. 41, 466-470.

Matsui, M., Watanabe, T., Kamijo, N., Lapp, R. L. \& Jacobson, R. A. (1980). Acta Cryst. B36, 1081-1086.

Mizutani, U. (2011). Hume-Rothery Rules for Structurally Complex Alloy Phases. CRC Press-Taylor \& Francis Group: Boca Raton, FL.

Sekine, Y., Motokawa, R., Kozai, N., Ohnuki, T., Matsumura, D., Tsuji, T., Kawasaki, R. \& Akiyoshi, K. (2017). Sci. Reports 7, article number 2064.

Sheldrick, G. M. (2015). Acta Cryst. C71, 3-8.

Stahl, P. H. \& Wermuth, C. G. (2008). Eds. Handbook of Pharmaceutical Salts: Properties, Selection and Use. VHCA: Zurich.

Trifa, C., Bouhali, A., Boudaren, C., Bouacida, S. \& Bataille, T. (2007). Acta Cryst. E63, i102-i104.

Wahlberg, J. S., Baker, J. H., Vernon, R. W. \& Dewar, R. S. (1965). Geological Survey Bulletin report 1140-c.

\section{Figure 1}

Top, contents of the asymmetric unit of the 69:31 Ca:Sr species with non-H atoms shown as 50\% probability ellipsoids. Atom label M1 is the mixed metal site occupied by Ca1 and Sr1. Bottom, for the same structure, part of the onedimensional coordination polymer that extends in the crystallographic $\mathrm{c}$ direction. $\mathrm{H}$ atoms are omitted for clarity.

\section{Figure 2}

Top, contents of the asymmetric unit for the 73:27 Sr:Ba species, with non-H atoms shown as $50 \%$ probability ellipsoids. Atom label M1 is the mixed metal site occupied by $\mathrm{Sr} 1$ and Ba1. Bottom, for the same structure, part of the onedimensional coordination polymer that extends in the crystallographic $\mathrm{c}$ direction. $\mathrm{H}$ atoms are omitted for clarity.

\section{Figure 3}

Expansion of unit cell volume with increasing Sr content. For comparison, the unit cell volume of the 72.9:27.1 Sr:Ba species is $1529.04(11) \AA^{3}$.

\section{Figure 4}

Change in length of unit cell axes with increase in $\mathrm{Sr}$ content. All percentages are given as change from the $100 \% \mathrm{Ca}$ species. Black $=a$ axis, green $=b$ axis and blue $=c$ axis. 


\section{Figure 5}

Change in unit cell volume and length of unit cell axes with change in temperature for calcium salicylate. All percentages are given as change from the corresponding values at $123 \mathrm{~K}$. Black $=a$ axis, green $=b$ axis and blue $=c$ axis.

\section{Figure 6}

Packing diagram of $100 \%$ Ca structure viewed along the $c$ axis and hence down the length of the coordination polymers. Hydrogen bond contacts are drawn as dotted lines and hydrogen atoms are omitted for clarity. The purple ring highlights one area of the hydrophobic contacts discussed in the main text.

\section{Figure 7}

Aqueous solubility versus increasing Sr content, as measured by AA analysis of bulk powders. 


\section{Special details}

Geometry. All e.s.d.'s (except the e.s.d. in the dihedral angle between two 1.s. planes) are estimated using the full covariance matrix. The cell e.s.d.'s are taken into account individually in the estimation of e.s.d.'s in distances, angles and torsion angles; correlations between e.s.d.'s in cell parameters are only used when they are defined by crystal symmetry. An approximate (isotropic) treatment of cell e.s.d.'s is used for estimating e.s.d.'s involving l.s. planes.

Fractional atomic coordinates and isotropic or equivalent isotropic displacement parameters $\left(\AA^{2}\right)$

\begin{tabular}{lllll}
\hline & $x$ & $y$ & $z$ & $U_{\text {iss }} * / U_{\text {eq }}$ \\
\hline Ca1 & 0.5000 & $0.45083(3)$ & -0.2500 & $0.01114(11)$ \\
O1 & $0.25618(6)$ & $0.25378(9)$ & $0.26849(13)$ & $0.0177(2)$ \\
O2 & $0.37861(6)$ & $0.40061(8)$ & $0.26911(12)$ & $0.0153(2)$ \\
O3 & $0.45483(6)$ & $0.39842(8)$ & $0.03573(12)$ & $0.0148(2)$ \\
O1W & $0.39906(6)$ & $0.32390(9)$ & $-0.37242(13)$ & $0.0196(2)$ \\
C1 & $0.30186(8)$ & $0.18870(11)$ & $0.15844(16)$ & $0.0135(3)$ \\
C2 & $0.27321(9)$ & $0.07827(12)$ & $0.11389(17)$ & $0.0180(3)$ \\
H2 & 0.2225 & 0.0518 & 0.1550 & $0.022^{*}$ \\
C3 & $0.31936(9)$ & $0.00752(12)$ & $0.00916(18)$ & $0.0206(3)$ \\
H3 & 0.3005 & -0.0685 & -0.0191 & $0.025^{*}$ \\
C4 & $0.39285(9)$ & $0.04591(12)$ & $-0.05540(19)$ & $0.0201(3)$ \\
H4 & 0.4244 & -0.0039 & -0.1257 & $0.024^{*}$ \\
C5 & $0.41968(8)$ & $0.15710(11)$ & $-0.01649(17)$ & $0.0164(3)$ \\
H5 & 0.4692 & 0.1842 & -0.0630 & $0.020^{*}$ \\
C6 & $0.37466(8)$ & $0.23026(11)$ & $0.09084(16)$ & $0.0126(3)$ \\
C7 & $0.40414(8)$ & $0.34989(11)$ & $0.13286(16)$ & $0.0116(3)$ \\
H1O & $0.2805(13)$ & $0.3137(18)$ & $0.288(3)$ & $0.041(6)^{*}$ \\
H1W & $0.3577(10)$ & $0.2942(16)$ & $-0.336(2)$ & $0.037(5)^{*}$ \\
H2W & $0.3917(12)$ & $0.3364(16)$ & $-0.481(2)$ & $0.035(5)^{*}$ \\
& & & &
\end{tabular}

Atomic displacement parameters $\left(\AA^{2}\right)$

\begin{tabular}{lllllll}
\hline & $U^{11}$ & $U^{22}$ & $U^{33}$ & $U^{12}$ & $U^{13}$ & $U^{23}$ \\
\hline Ca1 & $0.01109(19)$ & $0.01165(18)$ & $0.01080(19)$ & 0.000 & $0.00189(13)$ & 0.000 \\
O1 & $0.0145(5)$ & $0.0186(5)$ & $0.0203(5)$ & $-0.0045(4)$ & $0.0060(4)$ & $-0.0023(4)$ \\
O2 & $0.0163(5)$ & $0.0156(5)$ & $0.0143(5)$ & $-0.0024(4)$ & $0.0034(4)$ & $-0.0028(4)$ \\
O3 & $0.0149(5)$ & $0.0144(4)$ & $0.0154(5)$ & $-0.0032(3)$ & $0.0037(4)$ & $0.0011(4)$ \\
O1W & $0.0171(6)$ & $0.0261(6)$ & $0.0156(5)$ & $-0.0084(4)$ & $0.0024(4)$ & $0.0007(4)$ \\
C1 & $0.0134(7)$ & $0.0158(6)$ & $0.0111(6)$ & $-0.0005(5)$ & $-0.0005(5)$ & $0.0016(5)$ \\
C2 & $0.0189(7)$ & $0.0187(7)$ & $0.0164(6)$ & $-0.0074(5)$ & $-0.0007(5)$ & $0.0035(5)$ \\
C3 & $0.0302(8)$ & $0.0141(6)$ & $0.0173(7)$ & $-0.0054(6)$ & $-0.0030(6)$ & $0.0004(6)$ \\
C4 & $0.0259(8)$ & $0.0161(7)$ & $0.0185(7)$ & $0.0022(5)$ & $0.0027(6)$ & $-0.0032(6)$ \\
C5 & $0.0154(7)$ & $0.0180(7)$ & $0.0158(6)$ & $0.0002(5)$ & $0.0023(5)$ & $0.0006(5)$ \\
C6 & $0.0141(6)$ & $0.0122(6)$ & $0.0116(6)$ & $-0.0014(5)$ & $-0.0012(5)$ & $0.0013(5)$ \\
C7 & $0.0098(6)$ & $0.0133(6)$ & $0.0116(6)$ & $0.0008(4)$ & $-0.0014(4)$ & $0.0015(5)$
\end{tabular}

Geometric parameters $\left(\hat{A},{ }^{\circ}\right)$

\begin{tabular}{llll}
\hline $\mathrm{Ca} 1-\mathrm{O} 1 W^{\mathrm{i}}$ & $2.3761(10)$ & $\mathrm{O} 3-\mathrm{C} 7$ & $1.2624(15)$ \\
$\mathrm{Ca} 1-\mathrm{O} 1 \mathrm{~W}$ & $2.3761(10)$ & $\mathrm{O} 3-\mathrm{Ca} 1^{\mathrm{ii}}$ & $2.4781(9)$ \\
$\mathrm{Ca} 1-\mathrm{O} 3$ & $2.4005(9)$ & $\mathrm{O} 1 \mathrm{~W}-\mathrm{H} 1 \mathrm{~W}$ & $0.819(15)$ \\
$\mathrm{Ca} 1-\mathrm{O} 3^{\mathrm{i}}$ & $2.4005(9)$ & $\mathrm{O} 1 \mathrm{~W}-\mathrm{H} 2 \mathrm{~W}$ & $0.842(15)$ \\
$\mathrm{Ca} 1-\mathrm{O} 3^{\mathrm{ii}}$ & $2.4781(9)$ & $\mathrm{C} 1-\mathrm{C} 2$ & $1.3924(18)$
\end{tabular}




\begin{tabular}{|c|c|c|c|}
\hline $\mathrm{Ca} 1-\mathrm{O} 3^{\mathrm{iii}}$ & $2.4781(9)$ & $\mathrm{C} 1-\mathrm{C} 6$ & $1.4016(18)$ \\
\hline $\mathrm{Ca} 1-\mathrm{O} 2^{\mathrm{ii}}$ & $2.6333(9)$ & $\mathrm{C} 2-\mathrm{C} 3$ & $1.383(2)$ \\
\hline $\mathrm{Ca} 1-\mathrm{O} 2^{\mathrm{iii}}$ & $2.6333(9)$ & $\mathrm{C} 2-\mathrm{H} 2$ & 0.9500 \\
\hline $\mathrm{Ca} 1-\mathrm{C}^{\mathrm{ii}}$ & $2.9041(13)$ & $\mathrm{C} 3-\mathrm{C} 4$ & $1.390(2)$ \\
\hline $\mathrm{Ca} 1-\mathrm{C} 7^{\mathrm{iii}}$ & $2.9041(13)$ & $\mathrm{C} 3-\mathrm{H} 3$ & 0.9500 \\
\hline $\mathrm{Ca} 1-\mathrm{Ca} 1^{\mathrm{ii}}$ & $3.9745(4)$ & $\mathrm{C} 4-\mathrm{C} 5$ & $1.3814(19)$ \\
\hline $\mathrm{Ca} 1-\mathrm{Ca} 1^{\mathrm{iv}}$ & $3.9745(4)$ & $\mathrm{C} 4-\mathrm{H} 4$ & 0.9500 \\
\hline $\mathrm{Ca} 1-\mathrm{H} 2 \mathrm{~W}$ & $2.789(17)$ & $\mathrm{C} 5-\mathrm{C} 6$ & $1.4013(18)$ \\
\hline $\mathrm{O} 1-\mathrm{C} 1$ & $1.3669(16)$ & $\mathrm{C} 5-\mathrm{H} 5$ & 0.9500 \\
\hline $\mathrm{O} 1-\mathrm{H} 1 \mathrm{O}$ & $0.81(2)$ & $\mathrm{C} 6-\mathrm{C} 7$ & $1.4898(17)$ \\
\hline $\mathrm{O} 2-\mathrm{C} 7$ & $1.2740(15)$ & $\mathrm{C} 7-\mathrm{Ca}^{\mathrm{ii}}$ & $2.9041(13)$ \\
\hline $\mathrm{O} 2-\mathrm{Ca}^{\mathrm{ii}}$ & $2.6333(9)$ & & \\
\hline $\mathrm{O} 1 \mathrm{~W}^{\mathrm{i}}-\mathrm{Ca} 1-\mathrm{O} 1 \mathrm{~W}$ & $104.19(5)$ & $\mathrm{O} 1 \mathrm{~W}^{\mathrm{i}}-\mathrm{Ca} 1-\mathrm{Ca} 1^{\mathrm{iv}}$ & $121.93(3)$ \\
\hline $\mathrm{O} 1 \mathrm{~W}^{\mathrm{i}}-\mathrm{Ca} 1-\mathrm{O} 3$ & $73.88(3)$ & $\mathrm{O} 1 \mathrm{~W}-\mathrm{Ca} 1-\mathrm{Ca}^{\mathrm{iv}}$ & $79.67(3)$ \\
\hline $\mathrm{O} 1 \mathrm{~W}-\mathrm{Ca} 1-\mathrm{O} 3$ & $88.24(3)$ & $\mathrm{O} 3-\mathrm{Ca} 1-\mathrm{Ca}^{\mathrm{iv}}$ & $161.97(2)$ \\
\hline $\mathrm{O} 1 \mathrm{~W}^{\mathrm{i}}-\mathrm{Ca} 1-\mathrm{O}^{\mathrm{i}}$ & $88.24(3)$ & $\mathrm{O} 3{ }^{\mathrm{i}}-\mathrm{Ca} 1-\mathrm{Ca}^{\mathrm{iv}}$ & $36.10(2)$ \\
\hline $\mathrm{O} 1 \mathrm{~W}-\mathrm{Ca} 1-\mathrm{O} 3^{\mathrm{i}}$ & $73.88(3)$ & $\mathrm{O} 33^{\mathrm{ii}}-\mathrm{Ca} 1-\mathrm{Ca}^{\mathrm{iv}}$ & $115.02(3)$ \\
\hline $\mathrm{O} 3-\mathrm{Ca} 1-\mathrm{O}^{\mathrm{i}}$ & $150.92(5)$ & $\mathrm{O} 3^{\mathrm{iii}}-\mathrm{Ca} 1-\mathrm{Ca}^{\mathrm{iv}}$ & $34.80(2)$ \\
\hline $\mathrm{O} 1 \mathrm{~W}^{\mathrm{i}}-\mathrm{Ca} 1-\mathrm{O}^{\mathrm{ii}}$ & $88.94(3)$ & $\mathrm{O} 2^{\mathrm{ii}}-\mathrm{Ca} 1-\mathrm{Ca}^{\mathrm{iv}}$ & $74.82(2)$ \\
\hline $\mathrm{O} 1 \mathrm{~W}-\mathrm{Ca} 1-\mathrm{O}^{3 \mathrm{ii}}$ & $151.41(3)$ & $\mathrm{O} 2^{\mathrm{iii}}-\mathrm{Ca} 1-\mathrm{Ca} 1^{\mathrm{iv}}$ & $83.84(2)$ \\
\hline $\mathrm{O} 3-\mathrm{Ca} 1-\mathrm{O} 3^{\mathrm{ii}}$ & $70.90(3)$ & $\mathrm{C} 7^{\mathrm{ii}}-\mathrm{Ca} 1-\mathrm{Ca}^{\mathrm{iv}}$ & $93.02(3)$ \\
\hline $\mathrm{O} 3^{\mathrm{i}}-\mathrm{Ca} 1-\mathrm{O} 3^{\mathrm{ii}}$ & $132.73(3)$ & $\mathrm{C} 7^{\mathrm{iii}}-\mathrm{Ca} 1-\mathrm{Ca}^{\mathrm{iv}}$ & $59.88(3)$ \\
\hline $\mathrm{O} 1 \mathrm{~W}^{\mathrm{i}}-\mathrm{Ca} 1-\mathrm{O} 3^{\mathrm{iii}}$ & $151.41(3)$ & $\mathrm{Ca} 1^{\mathrm{ii}}-\mathrm{Ca} 1-\mathrm{Ca} 1^{\mathrm{iv}}$ & $146.93(2)$ \\
\hline $\mathrm{O} 1 \mathrm{~W}-\mathrm{Ca} 1-\mathrm{O} 3^{\mathrm{iii}}$ & $88.94(3)$ & $\mathrm{O} 1 \mathrm{~W}^{\mathrm{i}}-\mathrm{Ca} 1-\mathrm{H} 2 \mathrm{~W}$ & $112.3(4)$ \\
\hline $\mathrm{O} 3-\mathrm{Ca} 1-\mathrm{O}^{\mathrm{iii}}$ & $132.73(3)$ & $\mathrm{O} 1 \mathrm{~W}-\mathrm{Ca} 1-\mathrm{H} 2 \mathrm{~W}$ & $16.4(3)$ \\
\hline $\mathrm{O} 3^{\mathrm{i}}-\mathrm{Ca} 1-\mathrm{O} 3^{\mathrm{iii}}$ & $70.90(3)$ & $\mathrm{O} 3-\mathrm{Ca} 1-\mathrm{H} 2 \mathrm{~W}$ & $104.1(3)$ \\
\hline $\mathrm{O} 3^{\mathrm{ii}}-\mathrm{Ca} 1-\mathrm{O}^{\mathrm{iii}}$ & $91.21(4)$ & $\mathrm{O} 33^{\mathrm{i}}-\mathrm{Ca} 1-\mathrm{H} 2 \mathrm{~W}$ & $61.3(4)$ \\
\hline $\mathrm{O} 1 \mathrm{~W}^{\mathrm{i}}-\mathrm{Ca} 1-\mathrm{O} 2^{\mathrm{ii}}$ & $84.30(3)$ & $\mathrm{O} 33^{\mathrm{ii}}-\mathrm{Ca} 1-\mathrm{H} 2 \mathrm{~W}$ & $156.4(4)$ \\
\hline $\mathrm{O} 1 \mathrm{~W}-\mathrm{Ca} 1-\mathrm{O} 2^{\mathrm{ii}}$ & $153.69(3)$ & $\mathrm{O} 33^{\mathrm{iii}}-\mathrm{Ca} 1-\mathrm{H} 2 \mathrm{~W}$ & $75.2(4)$ \\
\hline $\mathrm{O} 3-\mathrm{Ca} 1-\mathrm{O} 2^{\mathrm{ii}}$ & $118.07(3)$ & $\mathrm{O} 2^{\mathrm{ii}}-\mathrm{Ca} 1-\mathrm{H} 2 \mathrm{~W}$ & $137.7(3)$ \\
\hline $\mathrm{O} 3^{\mathrm{i}}-\mathrm{Ca} 1-\mathrm{O} 2^{\mathrm{ii}}$ & $81.68(3)$ & $\mathrm{O} 22^{\mathrm{iii}}-\mathrm{Ca} 1-\mathrm{H} 2 \mathrm{~W}$ & $82.7(4)$ \\
\hline $\mathrm{O} 3^{\mathrm{ii}}-\mathrm{Ca} 1-\mathrm{O} 2^{\mathrm{ii}}$ & $51.10(3)$ & $\mathrm{C} 7{ }^{\mathrm{ii}}-\mathrm{Ca} 1-\mathrm{H} 2 \mathrm{~W}$ & $153.4(3)$ \\
\hline $\mathrm{O} 3^{\mathrm{iii}}-\mathrm{Ca} 1-\mathrm{O} 2^{\mathrm{ii}}$ & $73.75(3)$ & $\mathrm{C} 7^{\mathrm{iii}}-\mathrm{Ca} 1-\mathrm{H} 2 \mathrm{~W}$ & $81.4(4)$ \\
\hline $\mathrm{O} 1 \mathrm{~W}^{\mathrm{i}}-\mathrm{Ca} 1-\mathrm{O} 2^{\mathrm{iii}}$ & $153.69(3)$ & $\mathrm{Ca} 1^{\mathrm{ii}}-\mathrm{Ca} 1-\mathrm{H} 2 \mathrm{~W}$ & $135.9(4)$ \\
\hline $\mathrm{O} 1 \mathrm{~W}-\mathrm{Ca} 1-\mathrm{O} 2^{\mathrm{iii}}$ & $84.30(3)$ & $\mathrm{Ca}^{\mathrm{iv}}-\mathrm{Ca} 1-\mathrm{H} 2 \mathrm{~W}$ & $63.3(3)$ \\
\hline $\mathrm{O} 3-\mathrm{Ca} 1-\mathrm{O} 2^{\mathrm{iii}}$ & $81.68(3)$ & $\mathrm{C} 1-\mathrm{O} 1-\mathrm{H} 1 \mathrm{O}$ & $107.4(15)$ \\
\hline $\mathrm{O} 3^{\mathrm{i}}-\mathrm{Ca} 1-\mathrm{O} 2^{\mathrm{iii}}$ & $118.07(3)$ & $\mathrm{C} 7-\mathrm{O} 2-\mathrm{Ca} 1^{\mathrm{ii}}$ & $88.94(7)$ \\
\hline $\mathrm{O} 3^{\mathrm{ii}}-\mathrm{Ca} 1-\mathrm{O} 2^{\mathrm{iii}}$ & $73.75(3)$ & $\mathrm{C} 7-\mathrm{O} 3-\mathrm{Ca} 1$ & $150.77(8)$ \\
\hline $\mathrm{O} 3^{\mathrm{iii}}-\mathrm{Ca} 1-\mathrm{O} 2^{\mathrm{iii}}$ & $51.10(3)$ & $\mathrm{C} 7-\mathrm{O} 3-\mathrm{Ca} 1^{\mathrm{ii}}$ & $96.42(7)$ \\
\hline $\mathrm{O} 2^{\mathrm{ii}}-\mathrm{Ca} 1-\mathrm{O} 2^{\mathrm{iii}}$ & $99.10(4)$ & $\mathrm{Ca} 1-\mathrm{O} 3-\mathrm{Ca}^{\mathrm{ii}}$ & $109.10(3)$ \\
\hline $\mathrm{O} 1 \mathrm{~W}^{\mathrm{i}}-\mathrm{Ca} 1-\mathrm{C}^{\mathrm{ii}}$ & $90.01(4)$ & $\mathrm{Ca} 1-\mathrm{O} 1 \mathrm{~W}-\mathrm{H} 1 \mathrm{~W}$ & $134.2(13)$ \\
\hline $\mathrm{O} 1 \mathrm{~W}-\mathrm{Ca} 1-\mathrm{C}^{\mathrm{ii}}$ & $165.80(4)$ & $\mathrm{Ca} 1-\mathrm{O} 1 \mathrm{~W}-\mathrm{H} 2 \mathrm{~W}$ & $110.8(13)$ \\
\hline $\mathrm{O} 3-\mathrm{Ca} 1-\mathrm{C}^{\mathrm{ii}}$ & $95.75(3)$ & $\mathrm{H} 1 \mathrm{~W}-\mathrm{O} 1 \mathrm{~W}-\mathrm{H} 2 \mathrm{~W}$ & $108.3(18)$ \\
\hline $\mathrm{O} 3^{\mathrm{i}}-\mathrm{Ca} 1-\mathrm{C} 7^{\mathrm{ii}}$ & $107.23(3)$ & $\mathrm{O} 1-\mathrm{C} 1-\mathrm{C} 2$ & $117.45(12)$ \\
\hline $\mathrm{O} 3^{\mathrm{ii}}-\mathrm{Ca} 1-\mathrm{C}^{\mathrm{ii}}$ & $25.59(3)$ & $\mathrm{O} 1-\mathrm{C} 1-\mathrm{C} 6$ & $122.09(11)$ \\
\hline $\mathrm{O} 3^{\mathrm{iii}}-\mathrm{Ca} 1-\mathrm{C}^{\mathrm{ii}}$ & $78.33(3)$ & $\mathrm{C} 2-\mathrm{C} 1-\mathrm{C} 6$ & $120.46(12)$ \\
\hline $\mathrm{O} 2^{\mathrm{ii}}-\mathrm{Ca} 1-\mathrm{C}^{\mathrm{ii}}$ & $26.01(3)$ & $\mathrm{C} 3-\mathrm{C} 2-\mathrm{C} 1$ & $119.33(13)$ \\
\hline $\mathrm{O} 2^{\mathrm{iii}}-\mathrm{Ca} 1-\mathrm{C}^{\mathrm{ii}}$ & $82.80(3)$ & $\mathrm{C} 3-\mathrm{C} 2-\mathrm{H} 2$ & 120.3 \\
\hline $\mathrm{O} 1 \mathrm{~W}^{\mathrm{i}}-\mathrm{Ca} 1-\mathrm{C}^{\mathrm{iii}}$ & $165.80(4)$ & $\mathrm{C} 1-\mathrm{C} 2-\mathrm{H} 2$ & 120.3 \\
\hline $\mathrm{O} 1 \mathrm{~W}-\mathrm{Ca} 1-\mathrm{C} 7^{\mathrm{iii}}$ & $90.01(4)$ & $\mathrm{C} 2-\mathrm{C} 3-\mathrm{C} 4$ & $121.08(13)$ \\
\hline $\mathrm{O} 3-\mathrm{Ca} 1-\mathrm{C}^{\mathrm{iii}}$ & $107.23(3)$ & $\mathrm{C} 2-\mathrm{C} 3-\mathrm{H} 3$ & 119.5 \\
\hline
\end{tabular}




\begin{tabular}{|c|c|c|c|}
\hline $\mathrm{O} 3^{\mathrm{i}}-\mathrm{Ca} 1-\mathrm{C} 7^{\mathrm{iii}}$ & $95.75(3)$ & $\mathrm{C} 4-\mathrm{C} 3-\mathrm{H} 3$ & 119.5 \\
\hline $\mathrm{O} 3^{\mathrm{ii}}-\mathrm{Ca} 1-\mathrm{C} 7^{\mathrm{iii}}$ & $78.33(3)$ & $\mathrm{C} 5-\mathrm{C} 4-\mathrm{C} 3$ & $119.50(13)$ \\
\hline $\mathrm{O}^{3 i i}-\mathrm{Ca} 1-\mathrm{C}^{\mathrm{iii}}$ & $25.59(3)$ & $\mathrm{C} 5-\mathrm{C} 4-\mathrm{H} 4$ & 120.3 \\
\hline $\mathrm{O} 2^{\mathrm{ii}}-\mathrm{Ca} 1-\mathrm{C} 7^{\mathrm{iii}}$ & $82.80(3)$ & $\mathrm{C} 3-\mathrm{C} 4-\mathrm{H} 4$ & 120.3 \\
\hline $\mathrm{O} 2^{\mathrm{iii}}-\mathrm{Ca} 1-\mathrm{C}^{\mathrm{iii}}$ & $26.01(3)$ & $\mathrm{C} 4-\mathrm{C} 5-\mathrm{C} 6$ & $120.68(13)$ \\
\hline $\mathrm{C} 7^{\mathrm{ii}}-\mathrm{Ca} 1-\mathrm{C} 7^{\mathrm{iii}}$ & $75.79(5)$ & $\mathrm{C} 4-\mathrm{C} 5-\mathrm{H} 5$ & 119.7 \\
\hline $\mathrm{O} 1 \mathrm{~W}^{\mathrm{i}}-\mathrm{Ca} 1-\mathrm{Ca}^{\mathrm{ii}}$ & $79.68(3)$ & $\mathrm{C} 6-\mathrm{C} 5-\mathrm{H} 5$ & 119.7 \\
\hline $\mathrm{O} 1 \mathrm{~W}-\mathrm{Ca} 1-\mathrm{Ca}^{\mathrm{ii}}$ & $121.93(3)$ & $\mathrm{C} 5-\mathrm{C} 6-\mathrm{C} 1$ & $118.88(12)$ \\
\hline $\mathrm{O} 3-\mathrm{Ca} 1-\mathrm{Ca}^{\mathrm{ii}}$ & $36.10(2)$ & $\mathrm{C} 5-\mathrm{C} 6-\mathrm{C} 7$ & $120.39(12)$ \\
\hline $\mathrm{O}^{3}-\mathrm{Ca} 1-\mathrm{Ca}^{\mathrm{ii}}$ & $161.97(2)$ & $\mathrm{C} 1-\mathrm{C} 6-\mathrm{C} 7$ & $120.73(11)$ \\
\hline $\mathrm{O} 3^{\mathrm{ii}}-\mathrm{Ca} 1-\mathrm{Ca} 1^{\mathrm{ii}}$ & $34.80(2)$ & $\mathrm{O} 3-\mathrm{C} 7-\mathrm{O} 2$ & $121.12(11)$ \\
\hline $\mathrm{O} 3^{\mathrm{iii}}-\mathrm{Ca} 1-\mathrm{Ca} 1^{\mathrm{ii}}$ & $115.02(3)$ & $\mathrm{O} 3-\mathrm{C} 7-\mathrm{C} 6$ & $119.88(11)$ \\
\hline $\mathrm{O} 2^{\mathrm{ii}}-\mathrm{Ca} 1-\mathrm{Ca} 1^{\mathrm{ii}}$ & $83.84(2)$ & $\mathrm{O} 2-\mathrm{C} 7-\mathrm{C} 6$ & $118.98(11)$ \\
\hline $\mathrm{O} 2^{\mathrm{iii}}-\mathrm{Ca} 1-\mathrm{Ca} 1^{\mathrm{ii}}$ & $74.82(2)$ & $\mathrm{O} 3-\mathrm{C} 7-\mathrm{Ca}^{\mathrm{ii}}$ & $57.99(6)$ \\
\hline $\mathrm{C} 7^{\mathrm{ii}}-\mathrm{Ca} 1-\mathrm{Ca}^{\mathrm{ii}}$ & $59.88(3)$ & $\mathrm{O} 2-\mathrm{C} 7-\mathrm{Ca}^{\mathrm{ii}}$ & $65.04(7)$ \\
\hline $\mathrm{C}^{\mathrm{iii}}-\mathrm{Ca} 1-\mathrm{Ca} 1^{\mathrm{ii}}$ & $93.02(3)$ & $\mathrm{C} 6-\mathrm{C} 7-\mathrm{Ca} 1^{\mathrm{ii}}$ & $164.53(9)$ \\
\hline $\mathrm{O} 1-\mathrm{C} 1-\mathrm{C} 2-\mathrm{C} 3$ & $-177.15(12)$ & $\mathrm{Ca}^{\mathrm{ii}}-\mathrm{O} 3-\mathrm{C} 7-\mathrm{O} 2$ & $16.52(13)$ \\
\hline $\mathrm{C} 6-\mathrm{C} 1-\mathrm{C} 2-\mathrm{C} 3$ & $3.2(2)$ & $\mathrm{Ca} 1-\mathrm{O} 3-\mathrm{C} 7-\mathrm{C} 6$ & $46.8(2)$ \\
\hline $\mathrm{C} 1-\mathrm{C} 2-\mathrm{C} 3-\mathrm{C} 4$ & $-1.5(2)$ & $\mathrm{Ca} 1^{\mathrm{ii}}-\mathrm{O} 3-\mathrm{C} 7-\mathrm{C} 6$ & $-162.11(10)$ \\
\hline $\mathrm{C} 2-\mathrm{C} 3-\mathrm{C} 4-\mathrm{C} 5$ & $-0.9(2)$ & $\mathrm{Ca} 1-\mathrm{O} 3-\mathrm{C} 7-\mathrm{Ca}^{\mathrm{ii}}$ & $-151.07(18)$ \\
\hline $\mathrm{C} 3-\mathrm{C} 4-\mathrm{C} 5-\mathrm{C} 6$ & $1.6(2)$ & $\mathrm{Ca} 1^{\mathrm{ii}}-\mathrm{O} 2-\mathrm{C} 7-\mathrm{O} 3$ & $-15.42(12)$ \\
\hline $\mathrm{C} 4-\mathrm{C} 5-\mathrm{C} 6-\mathrm{C} 1$ & $0.08(19)$ & $\mathrm{Ca}^{\mathrm{ii}}-\mathrm{O} 2-\mathrm{C} 7-\mathrm{C} 6$ & $163.22(10)$ \\
\hline $\mathrm{C} 4-\mathrm{C} 5-\mathrm{C} 6-\mathrm{C} 7$ & $179.75(12)$ & $\mathrm{C} 5-\mathrm{C} 6-\mathrm{C} 7-\mathrm{O} 3$ & $20.62(18)$ \\
\hline $\mathrm{O} 1-\mathrm{C} 1-\mathrm{C} 6-\mathrm{C} 5$ & $177.86(11)$ & $\mathrm{C} 1-\mathrm{C} 6-\mathrm{C} 7-\mathrm{O} 3$ & $-159.71(12)$ \\
\hline $\mathrm{C} 2-\mathrm{C} 1-\mathrm{C} 6-\mathrm{C} 5$ & $-2.53(19)$ & $\mathrm{C} 5-\mathrm{C} 6-\mathrm{C} 7-\mathrm{O} 2$ & $-158.04(12)$ \\
\hline $\mathrm{O} 1-\mathrm{C} 1-\mathrm{C} 6-\mathrm{C} 7$ & $-1.81(19)$ & $\mathrm{C} 1-\mathrm{C} 6-\mathrm{C} 7-\mathrm{O} 2$ & $21.63(18)$ \\
\hline $\mathrm{C} 2-\mathrm{C} 1-\mathrm{C} 6-\mathrm{C} 7$ & $177.80(12)$ & $\mathrm{C} 5-\mathrm{C} 6-\mathrm{C} 7-\mathrm{Ca}^{\mathrm{ii}}$ & $-56.9(4)$ \\
\hline $\mathrm{Ca} 1-\mathrm{O} 3-\mathrm{C} 7-\mathrm{O} 2$ & $-134.55(14)$ & $\mathrm{C} 1-\mathrm{C} 6-\mathrm{C} 7-\mathrm{Ca}^{\mathrm{ii}}$ & $122.8(3)$ \\
\hline
\end{tabular}

Symmetry codes: (i) $-x+1, y,-z-1 / 2$; (ii) $-x+1,-y+1,-z$; (iii) $x,-y+1, z-1 / 2$; (iv) $-x+1,-y+1,-z-1$.

Hydrogen-bond geometry $\left(\AA,{ }^{\circ}\right)$

\begin{tabular}{lllll}
\hline$D-\mathrm{H} \cdots A$ & $D-\mathrm{H}$ & $\mathrm{H} \cdots A$ & $D \cdots A$ & $D-\mathrm{H} \cdots A$ \\
\hline $\mathrm{O} 1-\mathrm{H} 1 O \cdots \mathrm{O} 2$ & $0.81(2)$ & $1.90(2)$ & $2.6256(13)$ & $148(2)$ \\
$\mathrm{O} 1 W-\mathrm{H} 1 W \cdots \mathrm{O} 1^{\mathrm{v}}$ & $0.82(2)$ & $2.03(2)$ & $2.8391(14)$ & $169(2)$ \\
$\mathrm{O} 1 W-\mathrm{H} 2 W \cdots \mathrm{O} 2^{\mathrm{vi}}$ & $0.84(2)$ & $2.05(2)$ & $2.8795(14)$ & $169(2)$ \\
\hline
\end{tabular}

Symmetry codes: (v) $-x+1 / 2,-y+1 / 2,-z$; (vi) $x, y, z-1$.

\section{(CaSr9010)}

\section{Crystal data}

$\mathrm{C}_{14} \mathrm{H}_{14} \mathrm{Ca}_{0.96} \mathrm{O}_{8} \mathrm{Sr}_{0.04}$

$M_{r}=352.27$

Monoclinic, $C 2 / c$

$a=16.4335$ (7) $\AA$

$b=11.4974$ (4) $\AA$

$c=7.6301(3) \AA$

$\beta=91.778(4)^{\circ}$

$V=1440.96(10) \AA^{3}$

$Z=4$
$F(000)=731$

$D_{\mathrm{x}}=1.624 \mathrm{Mg} \mathrm{m}^{-3}$

Mo $K \alpha$ radiation, $\lambda=0.71073 \AA$

Cell parameters from 11617 reflections

$\theta=3.4-29.5^{\circ}$

$\mu=0.61 \mathrm{~mm}^{-1}$

$T=150 \mathrm{~K}$

Prism, colourless

$0.30 \times 0.22 \times 0.08 \mathrm{~mm}$ 


\section{Data collection}

Oxford Diffraction Xcalibur E diffractometer

Radiation source: sealed tube $\omega$ scans

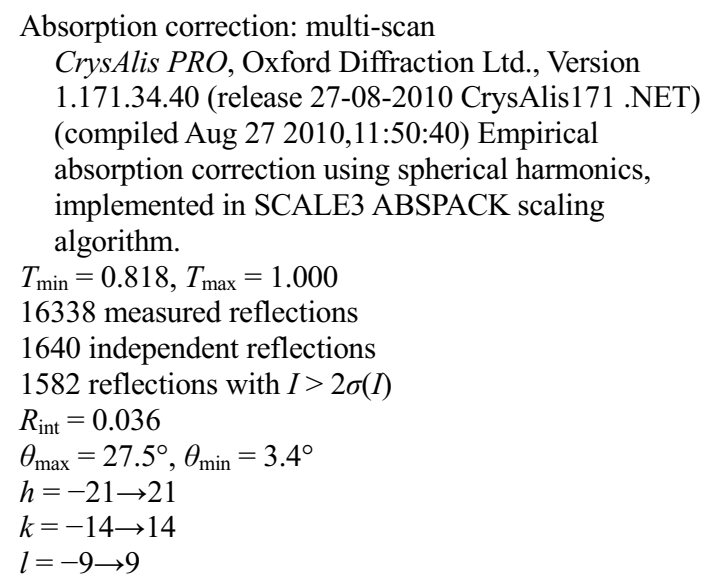

\section{Refinement}

Refinement on $F^{2}$

Least-squares matrix: full

$R\left[F^{2}>2 \sigma\left(F^{2}\right)\right]=0.021$

$w R\left(F^{2}\right)=0.055$

$S=1.07$

1640 reflections

118 parameters

0 restraints
Hydrogen site location: mixed

$\mathrm{H}$ atoms treated by a mixture of independent and constrained refinement

$w=1 /\left[\sigma^{2}\left(F_{\mathrm{o}}^{2}\right)+(0.0272 P)^{2}+0.9927 P\right]$

where $P=\left(F_{\mathrm{o}}{ }^{2}+2 F_{\mathrm{c}}{ }^{2}\right) / 3$

$(\Delta / \sigma)_{\max }<0.001$

$\Delta \rho_{\max }=0.29 \mathrm{e} \AA^{-3}$

$\Delta \rho_{\min }=-0.21$ e $\AA^{-3}$

\section{Special details}

Geometry. All e.s.d.'s (except the e.s.d. in the dihedral angle between two 1.s. planes) are estimated using the full covariance matrix. The cell e.s.d.'s are taken into account individually in the estimation of e.s.d.'s in distances, angles and torsion angles; correlations between e.s.d.'s in cell parameters are only used when they are defined by crystal symmetry. An approximate (isotropic) treatment of cell e.s.d.'s is used for estimating e.s.d.'s involving l.s. planes.

Fractional atomic coordinates and isotropic or equivalent isotropic displacement parameters $\left(\AA^{2}\right)$

\begin{tabular}{llllll}
\hline & $x$ & $y$ & $z$ & $U_{\text {iso }} * U_{\text {eq }}$ & Occ. $(<1)$ \\
\hline Sr1 & 0.5000 & $0.44977(2)$ & -0.2500 & $0.01035(10)$ & $0.041(2)$ \\
Ca1 & 0.5000 & $0.44977(2)$ & -0.2500 & $0.01035(10)$ & $0.959(2)$ \\
O1 & $0.25621(5)$ & $0.25393(7)$ & $0.26840(10)$ & $0.01890(18)$ & $0.01685(18)$ \\
O2 & $0.37837(4)$ & $0.40042(6)$ & $0.26872(9)$ & $0.01647(17)$ & $0.02102(19)$ \\
O3 & $0.45479(4)$ & $0.39796(6)$ & $0.03611(10)$ & $0.0145(2)$ & $0.0187(2)$ \\
O1W & $0.39881(5)$ & $0.32319(7)$ & $-0.37328(11)$ & $0.022^{*}$ & $0.0216(2)$ \\
C1 & $0.30173(6)$ & $0.18873(9)$ & $0.15848(13)$ & $0.026^{*}$ & $0.0215(2)$ \\
C2 & $0.27303(7)$ & $0.07831(9)$ & $0.11382(14)$ & $0.026^{*}$ \\
H2 & 0.2222 & 0.0520 & 0.1548 & $0.0176(2)$ & \\
C3 & $0.31912(7)$ & $0.00729(9)$ & $0.00925(15)$ & $0.021^{*}$ \\
H3 & 0.3002 & -0.0687 & -0.0189 & $0.0136(2)$ \\
C4 & $0.39272(7)$ & $0.04548(9)$ & $-0.05531(15)$ & $0.0129(2)$ \\
H4 & 0.4243 & -0.0044 & -0.1255 & $0.042(5)^{*}$ \\
C5 & $0.41952(7)$ & $0.15686(9)$ & $-0.01624(14)$ & $0.044(5)^{*}$ \\
H5 & 0.4690 & 0.1840 & -0.0627 &
\end{tabular}


Atomic displacement parameters $\left(\AA^{2}\right)$

\begin{tabular}{lllllll}
\hline & $U^{11}$ & $U^{22}$ & $U^{33}$ & $U^{12}$ & $U^{13}$ & $U^{23}$ \\
\hline Sr1 & $0.01016(15)$ & $0.01078(14)$ & $0.01022(15)$ & 0.000 & $0.00204(9)$ & 0.000 \\
Ca1 & $0.01016(15)$ & $0.01078(14)$ & $0.01022(15)$ & 0.000 & $0.00204(9)$ & 0.000 \\
O1 & $0.0163(4)$ & $0.0196(4)$ & $0.0212(4)$ & $-0.0039(3)$ & $0.0068(3)$ & $-0.0021(3)$ \\
O2 & $0.0179(4)$ & $0.0163(4)$ & $0.0165(4)$ & $-0.0021(3)$ & $0.0038(3)$ & $-0.0025(3)$ \\
O3 & $0.0163(4)$ & $0.0154(4)$ & $0.0180(4)$ & $-0.0034(3)$ & $0.0046(3)$ & $0.0007(3)$ \\
O1W & $0.0187(4)$ & $0.0272(4)$ & $0.0173(4)$ & $-0.0072(3)$ & $0.0031(3)$ & $0.0015(3)$ \\
C1 & $0.0151(5)$ & $0.0161(5)$ & $0.0124(4)$ & $-0.0009(4)$ & $-0.0004(4)$ & $0.0020(4)$ \\
C2 & $0.0205(5)$ & $0.0183(5)$ & $0.0172(5)$ & $-0.0071(4)$ & $-0.0006(4)$ & $0.0027(4)$ \\
C3 & $0.0317(6)$ & $0.0147(5)$ & $0.0183(5)$ & $-0.0062(4)$ & $-0.0022(4)$ & $-0.0002(4)$ \\
C4 & $0.0278(6)$ & $0.0169(5)$ & $0.0201(5)$ & $0.0023(4)$ & $0.0025(4)$ & $-0.0036(4)$ \\
C5 & $0.0179(5)$ & $0.0180(5)$ & $0.0171(5)$ & $-0.0002(4)$ & $0.0024(4)$ & $0.0001(4)$ \\
C6 & $0.0144(5)$ & $0.0131(4)$ & $0.0131(4)$ & $-0.0013(4)$ & $-0.0002(4)$ & $0.0009(4)$ \\
C7 & $0.0110(4)$ & $0.0134(4)$ & $0.0142(4)$ & $0.0004(3)$ & $-0.0004(3)$ & $0.0012(3)$ \\
\end{tabular}

Geometric parameters $\left(\AA,{ }^{\circ}\right)$

\begin{tabular}{|c|c|c|c|}
\hline $\mathrm{Sr} 1-\mathrm{O}_{1} \mathrm{~W}^{\mathrm{i}}$ & $2.3814(8)$ & $\mathrm{O} 3-\mathrm{C} 7$ & $1.2620(12)$ \\
\hline $\mathrm{Sr} 1-\mathrm{O} 1 \mathrm{~W}$ & $2.3814(8)$ & $\mathrm{O} 3-\mathrm{Ca}^{\mathrm{ii}}$ & $2.4910(7)$ \\
\hline $\mathrm{Sr} 1-\mathrm{O}^{\mathrm{i}}$ & $2.4027(7)$ & $\mathrm{O} 3-\mathrm{Sr} 1^{\mathrm{ii}}$ & $2.4910(7)$ \\
\hline $\mathrm{Sr} 1-\mathrm{O} 3$ & $2.4027(7)$ & $\mathrm{O} 1 \mathrm{~W}-\mathrm{H} 1 \mathrm{~W}$ & $0.864(19)$ \\
\hline $\mathrm{Sr} 1-\mathrm{O} 3^{\mathrm{ii}}$ & $2.4910(7)$ & $\mathrm{O} 1 \mathrm{~W}-\mathrm{H} 2 \mathrm{~W}$ & $0.853(18)$ \\
\hline $\mathrm{Sr} 1-\mathrm{O} 3^{\mathrm{iii}}$ & $2.4910(7)$ & $\mathrm{C} 1-\mathrm{C} 2$ & $1.3929(14)$ \\
\hline $\mathrm{Sr} 1-\mathrm{O} 2^{\mathrm{ii}}$ & $2.6458(7)$ & $\mathrm{C} 1-\mathrm{C} 6$ & $1.4002(14)$ \\
\hline $\mathrm{Sr} 1-\mathrm{O} 2^{\mathrm{iii}}$ & $2.6458(7)$ & $\mathrm{C} 2-\mathrm{C} 3$ & $1.3838(16)$ \\
\hline $\mathrm{Sr} 1-\mathrm{C} 7^{\mathrm{ii}}$ & $2.9198(10)$ & $\mathrm{C} 2-\mathrm{H} 2$ & 0.9500 \\
\hline $\mathrm{Sr} 1-\mathrm{C}^{\mathrm{iii}}$ & $2.9198(10)$ & $\mathrm{C} 3-\mathrm{C} 4$ & $1.3911(17)$ \\
\hline $\mathrm{Sr} 1-\mathrm{Sr} 1^{\mathrm{ii}}$ & $3.9861(2)$ & $\mathrm{C} 3-\mathrm{H} 3$ & 0.9500 \\
\hline $\mathrm{Sr} 1-\mathrm{Ca} 1^{\mathrm{ii}}$ & $3.9861(2)$ & $\mathrm{C} 4-\mathrm{C} 5$ & $1.3836(15)$ \\
\hline $\mathrm{Sr} 1-\mathrm{H} 2 \mathrm{~W}$ & $2.807(17)$ & $\mathrm{C} 4-\mathrm{H} 4$ & 0.9500 \\
\hline $\mathrm{O} 1-\mathrm{C} 1$ & $1.3652(12)$ & $\mathrm{C} 5-\mathrm{C} 6$ & $1.4007(14)$ \\
\hline $\mathrm{O} 1-\mathrm{H} 1 \mathrm{O}$ & $0.872(18)$ & $\mathrm{C} 5-\mathrm{H} 5$ & 0.9500 \\
\hline $\mathrm{O} 2-\mathrm{C} 7$ & $1.2724(12)$ & $\mathrm{C} 6-\mathrm{C} 7$ & $1.4878(13)$ \\
\hline $\mathrm{O} 2-\mathrm{Ca} 1^{\mathrm{ii}}$ & $2.6458(7)$ & $\mathrm{C} 7-\mathrm{Ca} 1^{\mathrm{ii}}$ & $2.9197(10)$ \\
\hline $\mathrm{O} 2-\mathrm{Sr}^{\mathrm{ii}}$ & $2.6458(7)$ & $\mathrm{C} 7-\mathrm{Sr} 1^{\mathrm{ii}}$ & $2.9197(10)$ \\
\hline $\mathrm{O} 1 \mathrm{~W}^{\mathrm{i}}-\mathrm{Sr} 1-\mathrm{O} 1 \mathrm{~W}$ & $104.66(4)$ & $\mathrm{O} 3^{\mathrm{iii}}-\mathrm{Sr} 1-\mathrm{Ca}^{\mathrm{ii}}$ & $114.49(2)$ \\
\hline $\mathrm{O} 1 \mathrm{~W}^{\mathrm{i}}-\mathrm{Sr} 1-\mathrm{O}^{\mathrm{i}}$ & $88.60(3)$ & $\mathrm{O} 2^{\mathrm{ii}}-\mathrm{Sr} 1-\mathrm{Ca} 1^{\mathrm{ii}}$ & $83.423(17)$ \\
\hline $\mathrm{O} 1 \mathrm{~W}-\mathrm{Sr} 1-\mathrm{O}^{3}{ }^{\mathrm{i}}$ & $73.82(3)$ & $\mathrm{O} 2^{\mathrm{iii}}-\mathrm{Sr} 1-\mathrm{Ca} 1^{\mathrm{ii}}$ & $74.766(17)$ \\
\hline $\mathrm{O} 1 \mathrm{~W}^{\mathrm{i}}-\mathrm{Sr} 1-\mathrm{O} 3$ & $73.82(3)$ & $\mathrm{C} 7^{\mathrm{ii}}-\mathrm{Sr} 1-\mathrm{Ca} 1^{\mathrm{ii}}$ & $59.63(2)$ \\
\hline $\mathrm{O} 1 \mathrm{~W}-\mathrm{Sr} 1-\mathrm{O} 3$ & $88.60(3)$ & $\mathrm{C} 7^{\mathrm{iii}}-\mathrm{Sr} 1-\mathrm{Ca} 1^{\mathrm{ii}}$ & $92.72(2)$ \\
\hline $\mathrm{O} 3{ }^{\mathrm{i}}-\mathrm{Sr} 1-\mathrm{O} 3$ & $151.29(4)$ & $\mathrm{Sr} 1^{\mathrm{ii}}-\mathrm{Sr} 1-\mathrm{Ca} 1^{\mathrm{ii}}$ & $0.000(7)$ \\
\hline $\mathrm{O} 1 \mathrm{~W}^{\mathrm{i}}-\mathrm{Sr} 1-\mathrm{O}^{\mathrm{ii}}$ & $88.87(3)$ & $\mathrm{O} 1 \mathrm{~W}^{\mathrm{i}}-\mathrm{Sr} 1-\mathrm{H} 2 \mathrm{~W}$ & $112.7(3)$ \\
\hline $\mathrm{O} 1 \mathrm{~W}-\mathrm{Sr} 1-\mathrm{O} 3^{\mathrm{ii}}$ & $151.49(3)$ & $\mathrm{O} 1 \mathrm{~W}-\mathrm{Sr} 1-\mathrm{H} 2 \mathrm{~W}$ & $16.4(4)$ \\
\hline $\mathrm{O} 3^{\mathrm{i}}-\mathrm{Sr} 1-\mathrm{O}^{\mathrm{ii}}$ & $132.47(3)$ & $\mathrm{O} 3^{\mathrm{i}}-\mathrm{Sr} 1-\mathrm{H} 2 \mathrm{~W}$ & $61.1(4)$ \\
\hline $\mathrm{O} 3-\mathrm{Sr} 1-\mathrm{O}^{\mathrm{ii}}$ & $70.93(3)$ & $\mathrm{O} 3-\mathrm{Sr} 1-\mathrm{H} 2 \mathrm{~W}$ & $104.5(4)$ \\
\hline $\mathrm{O} 1 \mathrm{~W}^{\mathrm{i}}-\mathrm{Sr} 1-\mathrm{O}^{\mathrm{iii}}$ & $151.49(3)$ & $\mathrm{O} 33^{\mathrm{ii}}-\mathrm{Sr} 1-\mathrm{H} 2 \mathrm{~W}$ & $156.3(3)$ \\
\hline $\mathrm{O} 1 \mathrm{~W}-\mathrm{Sr} 1-\mathrm{O} 3^{\mathrm{iii}}$ & $88.87(3)$ & $\mathrm{O} 33^{\mathrm{iii}}-\mathrm{Sr} 1-\mathrm{H} 2 \mathrm{~W}$ & $75.2(3)$ \\
\hline
\end{tabular}




\begin{tabular}{|c|c|c|c|}
\hline $\mathrm{O} 3^{\mathrm{i}}-\mathrm{Sr} 1-\mathrm{O} 3^{\mathrm{iii}}$ & $70.93(3)$ & $\mathrm{O} 2^{\mathrm{ii}}-\mathrm{Sr} 1-\mathrm{H} 2 \mathrm{~W}$ & $137.5(4)$ \\
\hline $\mathrm{O} 3-\mathrm{Sr} 1-\mathrm{O} 3^{\mathrm{iii}}$ & $132.47(3)$ & $\mathrm{O} 22^{\mathrm{iii}}-\mathrm{Sr} 1-\mathrm{H} 2 \mathrm{~W}$ & $82.7(3)$ \\
\hline $\mathrm{O} 3^{\mathrm{ii}}-\mathrm{Sr} 1-\mathrm{O} 3^{\mathrm{iii}}$ & $90.69(4)$ & $\mathrm{C}^{\mathrm{ii}}-\mathrm{Sr} 1-\mathrm{H} 2 \mathrm{~W}$ & $153.0(4)$ \\
\hline $\mathrm{O} 1 \mathrm{~W}^{\mathrm{i}}-\mathrm{Sr} 1-\mathrm{O} 2^{\mathrm{ii}}$ & $84.25(3)$ & $\mathrm{C}^{\mathrm{iii}}-\mathrm{Sr} 1-\mathrm{H} 2 \mathrm{~W}$ & $81.4(3)$ \\
\hline $\mathrm{O} 1 \mathrm{~W}-\mathrm{Sr} 1-\mathrm{O} 2^{\mathrm{ii}}$ & $153.63(3)$ & $\mathrm{Sr} 1{ }^{\mathrm{ii}}-\mathrm{Sr} 1-\mathrm{H} 2 \mathrm{~W}$ & $136.3(4)$ \\
\hline $\mathrm{O} 3^{\mathrm{i}}-\mathrm{Sr} 1-\mathrm{O} 2^{\mathrm{ii}}$ & $81.77(2)$ & $\mathrm{Ca} 1^{\mathrm{ii}}-\mathrm{Sr} 1-\mathrm{H} 2 \mathrm{~W}$ & $136.3(4)$ \\
\hline $\mathrm{O} 3-\mathrm{Sr} 1-\mathrm{O} 2^{\mathrm{ii}}$ & $117.77(2)$ & $\mathrm{C} 1-\mathrm{O} 1-\mathrm{H} 1 \mathrm{O}$ & $106.9(12)$ \\
\hline $\mathrm{O} 3^{\mathrm{ii}}-\mathrm{Sr} 1-\mathrm{O} 2^{\mathrm{ii}}$ & $50.76(2)$ & $\mathrm{C} 7-\mathrm{O} 2-\mathrm{Ca} 1^{\mathrm{ii}}$ & $89.20(6)$ \\
\hline 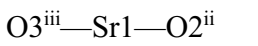 & $73.59(2)$ & $\mathrm{C} 7-\mathrm{O} 2-\mathrm{Sr}^{\mathrm{ii}}$ & $89.20(6)$ \\
\hline $\mathrm{O} 1 \mathrm{~W}^{\mathrm{i}}-\mathrm{Sr} 1-\mathrm{O} 2^{\mathrm{iii}}$ & $153.63(3)$ & $\mathrm{Ca} 1^{\mathrm{ii}}-\mathrm{O} 2-\mathrm{Sr} 1^{\mathrm{ii}}$ & 0.0 \\
\hline $\mathrm{O} 1 \mathrm{~W}-\mathrm{Sr} 1-\mathrm{O} 2^{\mathrm{iii}}$ & $84.25(3)$ & $\mathrm{C} 7-\mathrm{O} 3-\mathrm{Sr} 1$ & $150.54(7)$ \\
\hline $\mathrm{O} 3^{\mathrm{i}}-\mathrm{Sr} 1-\mathrm{O} 2^{\mathrm{iii}}$ & $117.76(2)$ & $\mathrm{C} 7-\mathrm{O} 3-\mathrm{Ca} 1^{\mathrm{ii}}$ & $96.64(6)$ \\
\hline $\mathrm{O} 3-\mathrm{Sr} 1-\mathrm{O} 2^{\mathrm{iii}}$ & $81.77(2)$ & $\mathrm{Sr} 1-\mathrm{O} 3-\mathrm{Ca}^{\mathrm{ii}}$ & 109.1 \\
\hline $\mathrm{O} 3^{\mathrm{ii}}-\mathrm{Sr} 1-\mathrm{O} 2^{\mathrm{iii}}$ & $73.59(2)$ & $\mathrm{C} 7-\mathrm{O} 3-\mathrm{Sr} 1^{\mathrm{ii}}$ & $96.64(6)$ \\
\hline 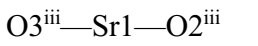 & $50.76(2)$ & $\mathrm{Sr} 1-\mathrm{O} 3-\mathrm{Sr}^{\mathrm{ii}}$ & $109.07(3)$ \\
\hline $\mathrm{O} 2^{\mathrm{ii}}-\mathrm{Sr} 1-\mathrm{O} 2^{\mathrm{iii}}$ & $98.76(3)$ & $\mathrm{Ca} 1^{\mathrm{ii}}-\mathrm{O} 3-\mathrm{Sr} 1^{\mathrm{ii}}$ & 0.0 \\
\hline $\mathrm{O} 1 \mathrm{~W}^{\mathrm{i}}-\mathrm{Sr} 1-\mathrm{C}^{\mathrm{ii}}$ & $89.90(3)$ & $\mathrm{Sr} 1-\mathrm{O} 1 \mathrm{~W}-\mathrm{H} 1 \mathrm{~W}$ & $133.8(11)$ \\
\hline $\mathrm{O} 1 \mathrm{~W}-\mathrm{Sr} 1-\mathrm{C} 7^{\mathrm{ii}}$ & $165.45(3)$ & $\mathrm{Sr} 1-\mathrm{O} 1 \mathrm{~W}-\mathrm{H} 2 \mathrm{~W}$ & $111.4(11)$ \\
\hline $\mathrm{O} 3^{\mathrm{i}}-\mathrm{Sr} 1-\mathrm{C}^{\mathrm{ii}}$ & $107.12(3)$ & $\mathrm{H} 1 \mathrm{~W}-\mathrm{O} 1 \mathrm{~W}-\mathrm{H} 2 \mathrm{~W}$ & $107.9(15)$ \\
\hline $\mathrm{O} 3-\mathrm{Sr} 1-\mathrm{C}^{\mathrm{ii}}$ & $95.60(3)$ & $\mathrm{O} 1-\mathrm{C} 1-\mathrm{C} 2$ & $117.57(9)$ \\
\hline $\mathrm{O} 3^{\mathrm{ii}}-\mathrm{Sr} 1-\mathrm{C}^{\mathrm{ii}}$ & $25.43(3)$ & $\mathrm{O} 1-\mathrm{C} 1-\mathrm{C} 6$ & $122.02(9)$ \\
\hline $\mathrm{O} 3^{\mathrm{iii}}-\mathrm{Sr} 1-\mathrm{C}^{\mathrm{ii}}$ & $78.00(3)$ & $\mathrm{C} 2-\mathrm{C} 1-\mathrm{C} 6$ & $120.40(10)$ \\
\hline $\mathrm{O} 2^{\mathrm{ii}}-\mathrm{Sr} 1-\mathrm{C}^{\mathrm{ii}}$ & $25.83(2)$ & $\mathrm{C} 3-\mathrm{C} 2-\mathrm{C} 1$ & $119.44(10)$ \\
\hline $\mathrm{O} 2^{\mathrm{iii}}-\mathrm{Sr} 1-\mathrm{C}^{\mathrm{ii}}$ & $82.58(3)$ & $\mathrm{C} 3-\mathrm{C} 2-\mathrm{H} 2$ & 120.3 \\
\hline $\mathrm{O} 1 \mathrm{~W}^{\mathrm{i}}-\mathrm{Sr} 1-\mathrm{C} 7^{\mathrm{iii}}$ & $165.45(3)$ & $\mathrm{C} 1-\mathrm{C} 2-\mathrm{H} 2$ & 120.3 \\
\hline $\mathrm{O} 1 \mathrm{~W}-\mathrm{Sr} 1-\mathrm{C}^{\mathrm{iii}}$ & $89.90(3)$ & $\mathrm{C} 2-\mathrm{C} 3-\mathrm{C} 4$ & $121.03(10)$ \\
\hline $\mathrm{O} 3^{\mathrm{i}}-\mathrm{Sr} 1-\mathrm{C} 7^{\mathrm{iii}}$ & $95.60(3)$ & $\mathrm{C} 2-\mathrm{C} 3-\mathrm{H} 3$ & 119.5 \\
\hline $\mathrm{O} 3-\mathrm{Sr} 1-\mathrm{C} 7^{\mathrm{iii}}$ & $107.12(3)$ & $\mathrm{C} 4-\mathrm{C} 3-\mathrm{H} 3$ & 119.5 \\
\hline $\mathrm{O} 3^{\mathrm{ii}}-\mathrm{Sr} 1-\mathrm{C} 7^{\mathrm{iii}}$ & $78.00(3)$ & $\mathrm{C} 5-\mathrm{C} 4-\mathrm{C} 3$ & $119.35(10)$ \\
\hline $\mathrm{O} 3^{\mathrm{iii}}-\mathrm{Sr} 1-\mathrm{C}^{\mathrm{iii}}$ & $25.43(3)$ & $\mathrm{C} 5-\mathrm{C} 4-\mathrm{H} 4$ & 120.3 \\
\hline $\mathrm{O} 2^{\mathrm{ii}}-\mathrm{Sr} 1-\mathrm{C}^{\mathrm{iii}}$ & $82.58(3)$ & $\mathrm{C} 3-\mathrm{C} 4-\mathrm{H} 4$ & 120.3 \\
\hline 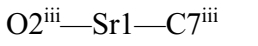 & $25.83(2)$ & $\mathrm{C} 4-\mathrm{C} 5-\mathrm{C} 6$ & $120.78(10)$ \\
\hline $\mathrm{C} 7^{\mathrm{ii}}-\mathrm{Sr} 1-\mathrm{C} 7^{\mathrm{iii}}$ & $75.55(4)$ & $\mathrm{C} 4-\mathrm{C} 5-\mathrm{H} 5$ & 119.6 \\
\hline $\mathrm{O} 1 \mathrm{~W}^{\mathrm{i}}-\mathrm{Sr} 1-\mathrm{Sr}^{\mathrm{ii}}$ & $79.62(2)$ & $\mathrm{C} 6-\mathrm{C} 5-\mathrm{H} 5$ & 119.6 \\
\hline $\mathrm{O} 1 \mathrm{~W}-\mathrm{Sr} 1-\mathrm{Sr} 1^{\mathrm{ii}}$ & $122.30(2)$ & $\mathrm{C} 1-\mathrm{C} 6-\mathrm{C} 5$ & $118.91(9)$ \\
\hline $\mathrm{O} 3^{\mathrm{i}}-\mathrm{Sr} 1-\mathrm{Sr} 1^{\mathrm{ii}}$ & $161.918(18)$ & $\mathrm{C} 1-\mathrm{C} 6-\mathrm{C} 7$ & $120.66(9)$ \\
\hline $\mathrm{O} 3-\mathrm{Sr} 1-\mathrm{Sr} 1^{\mathrm{ii}}$ & $36.203(17)$ & $\mathrm{C} 5-\mathrm{C} 6-\mathrm{C} 7$ & $120.43(9)$ \\
\hline $\mathrm{O}^{\mathrm{ii}}-\mathrm{Sr} 1-\mathrm{Sr} 1^{\mathrm{ii}}$ & $34.729(16)$ & $\mathrm{O} 3-\mathrm{C} 7-\mathrm{O} 2$ & $121.02(9)$ \\
\hline $\mathrm{O}^{\mathrm{iii}}-\mathrm{Sr} 1-\mathrm{Sr} 1^{\mathrm{ii}}$ & $114.49(2)$ & $\mathrm{O} 3-\mathrm{C} 7-\mathrm{C} 6$ & $119.87(9)$ \\
\hline $\mathrm{O} 2^{\mathrm{ii}}-\mathrm{Sr} 1-\mathrm{Sr} 1^{\mathrm{ii}}$ & $83.423(17)$ & $\mathrm{O} 2-\mathrm{C} 7-\mathrm{C} 6$ & $119.10(9)$ \\
\hline $\mathrm{O} 2^{\mathrm{iii}}-\mathrm{Sr} 1-\mathrm{Sr}^{\mathrm{ii}}$ & $74.766(17)$ & $\mathrm{O} 3-\mathrm{C} 7-\mathrm{Ca} 1^{\mathrm{ii}}$ & $57.93(5)$ \\
\hline $\mathrm{C} 7^{\mathrm{ii}}-\mathrm{Sr} 1-\mathrm{Sr} 1^{\mathrm{ii}}$ & $59.63(2)$ & $\mathrm{O} 2-\mathrm{C} 7-\mathrm{Ca} 1^{\mathrm{ii}}$ & $64.97(5)$ \\
\hline $\mathrm{C} 7^{\mathrm{iii}}-\mathrm{Sr} 1-\mathrm{Sr} 1^{\mathrm{ii}}$ & $92.72(2)$ & $\mathrm{C} 6-\mathrm{C} 7-\mathrm{Ca} 1^{\mathrm{ii}}$ & $164.70(7)$ \\
\hline $\mathrm{O} 1 \mathrm{~W}^{\mathrm{i}}-\mathrm{Sr} 1-\mathrm{Ca} 1^{\mathrm{ii}}$ & $79.62(2)$ & $\mathrm{O} 3-\mathrm{C} 7-\mathrm{Sr} 1^{\mathrm{ii}}$ & $57.93(5)$ \\
\hline $\mathrm{O} 1 \mathrm{~W}-\mathrm{Sr} 1-\mathrm{Ca} 1^{\mathrm{ii}}$ & $122.30(2)$ & $\mathrm{O} 2-\mathrm{C} 7-\mathrm{Sr} 1^{\mathrm{ii}}$ & $64.97(5)$ \\
\hline $\mathrm{O} 3{ }^{\mathrm{i}}-\mathrm{Sr} 1-\mathrm{Ca}^{\mathrm{ii}}$ & $161.918(18)$ & $\mathrm{C} 6-\mathrm{C} 7-\mathrm{Sr} 1^{\mathrm{ii}}$ & $164.70(7)$ \\
\hline $\mathrm{O} 3-\mathrm{Sr} 1-\mathrm{Ca} 1^{\mathrm{ii}}$ & $36.203(17)$ & $\mathrm{Ca} 1^{\mathrm{ii}}-\mathrm{C} 7-\mathrm{Sr} 1^{\mathrm{ii}}$ & 0.0 \\
\hline $\mathrm{O} 3^{\mathrm{ii}}-\mathrm{Sr} 1-\mathrm{Ca}^{\mathrm{ii}}$ & $34.729(16)$ & & \\
\hline $\mathrm{O} 1-\mathrm{C} 1-\mathrm{C} 2-\mathrm{C} 3$ & $-177.05(9)$ & $\mathrm{Sr}^{\mathrm{ii}}-\mathrm{O} 3-\mathrm{C} 7-\mathrm{Ca}^{\mathrm{ii}}$ & 0.0 \\
\hline $\mathrm{C} 6-\mathrm{C} 1-\mathrm{C} 2-\mathrm{C} 3$ & $3.26(15)$ & $\mathrm{Sr} 1-\mathrm{O} 3-\mathrm{C} 7-\mathrm{Sr}^{\mathrm{ii}}$ & $-151.02(14)$ \\
\hline $\mathrm{C} 1-\mathrm{C} 2-\mathrm{C} 3-\mathrm{C} 4$ & $-1.53(16)$ & $\mathrm{Ca} 1^{\mathrm{ii}}-\mathrm{O} 3-\mathrm{C} 7-\mathrm{Sr}^{\mathrm{ii}}$ & 0.0 \\
\hline
\end{tabular}




\begin{tabular}{|c|c|}
\hline $\mathrm{C} 2-\mathrm{C} 3-\mathrm{C} 4-\mathrm{C} 5$ & $-0.95(17)$ \\
\hline $\mathrm{C} 3-\mathrm{C} 4-\mathrm{C} 5-\mathrm{C} 6$ & $1.72(17)$ \\
\hline $\mathrm{O} 1-\mathrm{C} 1-\mathrm{C} 6-\mathrm{C} 5$ & $177.84(9)$ \\
\hline $\mathrm{C} 2-\mathrm{C} 1-\mathrm{C} 6-\mathrm{C} 5$ & $-2.49(15)$ \\
\hline $\mathrm{O} 1-\mathrm{C} 1-\mathrm{C} 6-\mathrm{C} 7$ & $-1.91(14)$ \\
\hline $\mathrm{C} 2-\mathrm{C} 1-\mathrm{C} 6-\mathrm{C} 7$ & $177.76(9)$ \\
\hline $\mathrm{C} 4-\mathrm{C} 5-\mathrm{C} 6-\mathrm{C} 1$ & $-0.02(15)$ \\
\hline $\mathrm{C} 4-\mathrm{C} 5-\mathrm{C} 6-\mathrm{C} 7$ & $179.73(10)$ \\
\hline $\mathrm{Sr} 1-\mathrm{O} 3-\mathrm{C} 7-\mathrm{O} 2$ & $-134.61(11)$ \\
\hline $\mathrm{Ca}^{\mathrm{ii}}-\mathrm{O} 3-\mathrm{C} 7-\mathrm{O} 2$ & $16.41(10)$ \\
\hline $\mathrm{Sr} 1^{\mathrm{ii}}-\mathrm{O} 3-\mathrm{C} 7-\mathrm{O} 2$ & $16.41(10)$ \\
\hline $\mathrm{Sr} 1-\mathrm{O} 3-\mathrm{C} 7-\mathrm{C} 6$ & $46.66(18)$ \\
\hline $\mathrm{Ca} 1^{\mathrm{ii}}-\mathrm{O} 3-\mathrm{C} 7-\mathrm{C} 6$ & $-162.32(7)$ \\
\hline $\mathrm{Sr} 1^{\mathrm{ii}}-\mathrm{O} 3-\mathrm{C} 7-\mathrm{C} 6$ & $-162.32(7)$ \\
\hline $\mathrm{Sr} 1-\mathrm{O} 3-\mathrm{C} 7-\mathrm{Ca}{ }^{\mathrm{ii}}$ & $-151.02(14)$ \\
\hline
\end{tabular}

\begin{tabular}{|c|c|}
\hline $\mathrm{Ca} 1{ }^{\mathrm{ii}}-\mathrm{O} 2-\mathrm{C} 7-\mathrm{O} 3$ & $-15.32(9)$ \\
\hline $\mathrm{Sr} 1{ }^{\mathrm{ii}}-\mathrm{O} 2-\mathrm{C} 7-\mathrm{O} 3$ & $-15.32(9)$ \\
\hline $\mathrm{Ca} 1^{\mathrm{ii}}-\mathrm{O} 2-\mathrm{C} 7-\mathrm{C} 6$ & $163.42(8)$ \\
\hline $\mathrm{Sr} 1^{\mathrm{ii}}-\mathrm{O} 2-\mathrm{C} 7-\mathrm{C} 6$ & $163.42(8)$ \\
\hline $\mathrm{Sr}^{\mathrm{ii}}-\mathrm{O} 2-\mathrm{C} 7-\mathrm{Ca}^{\mathrm{ii}}$ & 0.0 \\
\hline $\mathrm{Ca} 1^{\mathrm{ii}}-\mathrm{O} 2-\mathrm{C} 7-\mathrm{Sr}^{\mathrm{ii}}$ & 0.0 \\
\hline $\mathrm{C} 1-\mathrm{C} 6-\mathrm{C} 7-\mathrm{O} 3$ & $-159.70(9)$ \\
\hline $\mathrm{C} 5-\mathrm{C} 6-\mathrm{C} 7-\mathrm{O} 3$ & $20.55(14)$ \\
\hline $\mathrm{C} 1-\mathrm{C} 6-\mathrm{C} 7-\mathrm{O} 2$ & $21.55(14)$ \\
\hline $\mathrm{C} 5-\mathrm{C} 6-\mathrm{C} 7-\mathrm{O} 2$ & $-158.20(10)$ \\
\hline $\mathrm{C} 1-\mathrm{C} 6-\mathrm{C} 7-\mathrm{Ca}^{\mathrm{ii}}$ & $123.1(2)$ \\
\hline $\mathrm{C} 5-\mathrm{C} 6-\mathrm{C} 7-\mathrm{Ca} 1^{\mathrm{ii}}$ & $-56.7(3)$ \\
\hline $\mathrm{C} 1-\mathrm{C} 6-\mathrm{C} 7-\mathrm{Sr}^{\mathrm{ii}}$ & $123.1(2)$ \\
\hline $\mathrm{C} 5-\mathrm{C} 6-\mathrm{C} 7-\mathrm{Sr} 1^{\mathrm{ii}}$ & $-56.7(3)$ \\
\hline
\end{tabular}

Symmetry codes: (i) $-x+1, y,-z-1 / 2$; (ii) $-x+1,-y+1,-z$; (iii) $x,-y+1, z-1 / 2$.

Hydrogen-bond geometry $\left(\AA,{ }^{\circ}\right)$

\begin{tabular}{lllll}
\hline$D-\mathrm{H} \cdots A$ & $D-\mathrm{H}$ & $\mathrm{H} \cdots A$ & $D \cdots A$ & $D-\mathrm{H} \cdots A$ \\
\hline $\mathrm{O} 1-\mathrm{H} 1 O \cdots \mathrm{O} 2$ & $0.872(18)$ & $1.842(19)$ & $2.6204(10)$ & $147.6(16)$ \\
$\mathrm{O} 1 W-\mathrm{H} 1 W \cdots \mathrm{O} 1^{\mathrm{iv}}$ & $0.864(19)$ & $1.981(19)$ & $2.8361(11)$ & $169.8(16)$ \\
$\mathrm{O} 1 W-\mathrm{H} 2 W \cdots \mathrm{O} 2^{\mathrm{v}}$ & $0.853(18)$ & $2.043(18)$ & $2.8820(11)$ & $167.6(15)$
\end{tabular}

Symmetry codes: (iv) $-x+1 / 2,-y+1 / 2,-z$; (v) $x, y, z-1$.

\section{(CaSr8020)}

\section{Crystal data}

$\mathrm{C}_{14} \mathrm{H}_{14} \mathrm{Ca}_{0.92} \mathrm{O}_{8} \mathrm{Sr}_{0.08}$

$M_{r}=354.25$

Monoclinic, $C 2 / c$

$a=16.4344$ (3) $\AA$

$b=11.4853$ (2) $\AA$

$c=7.6417$ (2) $\AA$

$\beta=91.785(2)^{\circ}$

$V=1441.70(5) \AA^{3}$

$Z=4$

\section{Data collection}

Oxford Diffraction Xcalibur E diffractometer

Radiation source: sealed tube $\omega$ scans
$F(000)=734$

$D_{\mathrm{x}}=1.632 \mathrm{Mg} \mathrm{m}^{-3}$

Mo $K \alpha$ radiation, $\lambda=0.71073 \AA$

Cell parameters from 11379 reflections

$\theta=3.4-30.4^{\circ}$

$\mu=0.75 \mathrm{~mm}^{-1}$

$T=150 \mathrm{~K}$

Prism, colourless

$0.35 \times 0.28 \times 0.08 \mathrm{~mm}$

\footnotetext{
Absorption correction: multi-scan

CrysAlis PRO, Oxford Diffraction Ltd., Version

1.171.34.40 (release 27-08-2010 CrysAlis171 .NET)

(compiled Aug 27 2010,11:50:40) Empirical

absorption correction using spherical harmonics, implemented in SCALE3 ABSPACK scaling algorithm.

$T_{\min }=0.802, T_{\max }=1.000$

16552 measured reflections

1642 independent reflections

1568 reflections with $I>2 \sigma(I)$

$R_{\text {int }}=0.030$

$\theta_{\max }=27.5^{\circ}, \theta_{\min }=3.4^{\circ}$

$h=-21 \rightarrow 21$

$k=-14 \rightarrow 14$

$l=-9 \rightarrow 9$
} 


\section{Refinement}

Refinement on $F^{2}$

Least-squares matrix: full

$R\left[F^{2}>2 \sigma\left(F^{2}\right)\right]=0.021$

$w R\left(F^{2}\right)=0.053$

$S=1.08$

1642 reflections

118 parameters

0 restraints
Hydrogen site location: mixed

$\mathrm{H}$ atoms treated by a mixture of independent and constrained refinement

$w=1 /\left[\sigma^{2}\left(F_{\mathrm{o}}^{2}\right)+(0.0238 P)^{2}+1.0809 P\right]$

where $P=\left(F_{\mathrm{o}}{ }^{2}+2 F_{\mathrm{c}}{ }^{2}\right) / 3$

$(\Delta / \sigma)_{\max }<0.001$

$\Delta \rho_{\max }=0.31 \mathrm{e} \AA^{-3}$

$\Delta \rho_{\min }=-0.23$ e $\AA^{-3}$

\section{Special details}

Geometry. All e.s.d.'s (except the e.s.d. in the dihedral angle between two 1.s. planes) are estimated using the full covariance matrix. The cell e.s.d.'s are taken into account individually in the estimation of e.s.d.'s in distances, angles and torsion angles; correlations between e.s.d.'s in cell parameters are only used when they are defined by crystal symmetry. An approximate (isotropic) treatment of cell e.s.d.'s is used for estimating e.s.d.'s involving l.s. planes.

Fractional atomic coordinates and isotropic or equivalent isotropic displacement parameters $\left(\hat{A}^{2}\right)$

\begin{tabular}{llllll}
\hline & $x$ & $y$ & $z$ & $U_{\text {iso }} * U_{\text {eq }}$ & Occ. $(<1)$ \\
\hline Sr1 & 0.5000 & $0.44923(2)$ & -0.2500 & $0.01030(10)$ & $0.083(2)$ \\
Ca1 & 0.5000 & $0.44923(2)$ & -0.2500 & $0.01030(10)$ & $0.917(2)$ \\
O1 & $0.25630(5)$ & $0.25391(7)$ & $0.26823(11)$ & $0.01854(19)$ & $0.01658(18)$ \\
O2 & $0.37832(5)$ & $0.40026(7)$ & $0.26855(10)$ & $0.01642(18)$ & $0.0213(2)$ \\
O3 & $0.45479(5)$ & $0.39771(7)$ & $0.03630(10)$ & $0.0141(2)$ & $0.0183(2)$ \\
O1W & $0.39865(5)$ & $0.32276(8)$ & $-0.37365(12)$ & $0.022^{*}$ \\
C1 & $0.30175(6)$ & $0.18861(9)$ & $0.15851(13)$ & $0.0214(3)$ & $0.026^{*}$ \\
C2 & $0.27301(7)$ & $0.07823(10)$ & $0.11382(14)$ & $0.0215(3)$ \\
H2 & 0.2222 & 0.0519 & 0.1549 & $0.026^{*}$ \\
C3 & $0.31889(8)$ & $0.00733(10)$ & $0.00931(15)$ & $0.0171(2)$ \\
H3 & 0.2998 & -0.0687 & -0.0191 & $0.021^{*}$ \\
C4 & $0.39251(8)$ & $0.04538(10)$ & $-0.05506(16)$ & $0.0133(2)$ \\
H4 & 0.4240 & -0.0047 & -0.1251 & $0.0129(2)$ \\
C5 & $0.41944(7)$ & $0.15671(10)$ & $-0.01621(14)$ & $0.039(5)^{*}$ \\
H5 & 0.4689 & 0.1839 & -0.0627 & $0.043(5)^{*}$ \\
C6 & $0.37448(6)$ & $0.22991(9)$ & $0.09118(13)$ & $0.043(5)^{*}$ \\
C7 & $0.40395(6)$ & $0.34932(9)$ & $0.13318(13)$ & \\
H1O & $0.2813(11)$ & $0.3191(16)$ & $0.288(2)$ & $-0.339(2)$ & \\
H1W & $0.3551(12)$ & $0.2915(16)$ & $-0.481(3)$ & \\
H2W & $0.3914(11)$ & $0.3337(15)$ & & & \\
& & & & &
\end{tabular}

Atomic displacement parameters $\left(A^{2}\right)$

\begin{tabular}{lllllll}
\hline & $U^{11}$ & $U^{22}$ & $U^{33}$ & $U^{12}$ & $U^{13}$ & $U^{23}$ \\
\hline Sr1 & $0.00968(14)$ & $0.01105(15)$ & $0.01028(15)$ & 0.000 & $0.00222(9)$ & 0.000 \\
Ca1 & $0.00968(14)$ & $0.01105(15)$ & $0.01028(15)$ & 0.000 & $0.00222(9)$ & 0.000 \\
O1 & $0.0159(4)$ & $0.0194(4)$ & $0.0207(4)$ & $-0.0038(3)$ & $0.0065(3)$ & $-0.0023(3)$ \\
O2 & $0.0174(4)$ & $0.0164(4)$ & $0.0161(4)$ & $-0.0018(3)$ & $0.0038(3)$ & $-0.0029(3)$ \\
O3 & $0.0156(4)$ & $0.0154(4)$ & $0.0186(4)$ & $-0.0035(3)$ & $0.0049(3)$ & $0.0004(3)$ \\
O1W & $0.0182(4)$ & $0.0283(5)$ & $0.0176(4)$ & $-0.0069(3)$ & $0.0036(3)$ & $0.0018(3)$ \\
C1 & $0.0147(5)$ & $0.0159(5)$ & $0.0117(5)$ & $-0.0006(4)$ & $-0.0001(4)$ & $0.0020(4)$ \\
C2 & $0.0196(5)$ & $0.0187(5)$ & $0.0165(5)$ & $-0.0071(4)$ & $-0.0007(4)$ & $0.0029(4)$ \\
C3 & $0.0309(6)$ & $0.0146(5)$ & $0.0186(6)$ & $-0.0063(5)$ & $-0.0024(5)$ & $-0.0004(4)$ \\
C4 & $0.0280(6)$ & $0.0173(5)$ & $0.0193(6)$ & $0.0020(5)$ & $0.0025(5)$ & $-0.0036(4)$
\end{tabular}




$\begin{array}{lllllll}\text { C5 } & 0.0176(5) & 0.0173(5) & 0.0165(5) & -0.0002(4) & 0.0030(4) & 0.0000(4) \\ \text { C6 } & 0.0136(5) & 0.0130(5) & 0.0131(5) & -0.0010(4) & -0.0004(4) & 0.0010(4) \\ \text { C7 } & 0.0104(4) & 0.0142(5) & 0.0140(5) & 0.0006(4) & -0.0008(4) & 0.0013(4)\end{array}$

Geometric parameters $\left(A,{ }^{\circ}\right)$

\begin{tabular}{|c|c|c|c|}
\hline $\mathrm{Sr} 1-\mathrm{O} 1 \mathrm{~W}^{\mathrm{i}}$ & $2.3830(9)$ & $\mathrm{O} 3-\mathrm{C} 7$ & $1.2624(13)$ \\
\hline $\mathrm{Sr} 1-\mathrm{O} 1 \mathrm{~W}$ & $2.3830(9)$ & $\mathrm{O} 3-\mathrm{Ca}^{\mathrm{ii}}$ & $2.4968(8)$ \\
\hline $\mathrm{Sr} 1-\mathrm{O} 3$ & $2.4062(8)$ & $\mathrm{O} 3-\mathrm{Sr}^{\mathrm{ii}}$ & $2.4968(8)$ \\
\hline $\mathrm{Sr} 1-\mathrm{O}^{\mathrm{i}}$ & $2.4062(8)$ & $\mathrm{O} 1 \mathrm{~W}-\mathrm{H} 1 \mathrm{~W}$ & $0.85(2)$ \\
\hline $\mathrm{Sr} 1-\mathrm{O} 3^{\mathrm{ii}}$ & $2.4968(8)$ & $\mathrm{O} 1 \mathrm{~W}-\mathrm{H} 2 \mathrm{~W}$ & $0.836(19)$ \\
\hline $\mathrm{Sr} 1-\mathrm{O} 3^{\mathrm{iii}}$ & $2.4968(8)$ & $\mathrm{C} 1-\mathrm{C} 2$ & $1.3917(15)$ \\
\hline $\mathrm{Sr} 1-\mathrm{O} 2^{\mathrm{ii}}$ & $2.6506(8)$ & $\mathrm{C} 1-\mathrm{C} 6$ & $1.3989(15)$ \\
\hline $\mathrm{Sr} 1-\mathrm{O} 2^{\mathrm{iii}}$ & $2.6506(8)$ & $\mathrm{C} 2-\mathrm{C} 3$ & $1.3809(17)$ \\
\hline $\mathrm{Sr} 1-\mathrm{C}^{\mathrm{ii}}$ & $2.9247(11)$ & $\mathrm{C} 2-\mathrm{H} 2$ & 0.9500 \\
\hline $\mathrm{Sr} 1-\mathrm{C}^{\mathrm{iii}}$ & $2.9247(11)$ & $\mathrm{C} 3-\mathrm{C} 4$ & $1.3907(17)$ \\
\hline $\mathrm{Sr} 1-\mathrm{Sr} 1^{\mathrm{ii}}$ & $3.9949(2)$ & $\mathrm{C} 3-\mathrm{H} 3$ & 0.9500 \\
\hline $\mathrm{Sr} 1-\mathrm{Ca}^{\mathrm{ii}}$ & $3.9949(2)$ & $\mathrm{C} 4-\mathrm{C} 5$ & $1.3824(16)$ \\
\hline $\mathrm{Sr} 1-\mathrm{H} 2 \mathrm{~W}$ & $2.805(18)$ & $\mathrm{C} 4-\mathrm{H} 4$ & 0.9500 \\
\hline $\mathrm{O} 1-\mathrm{C} 1$ & $1.3647(13)$ & $\mathrm{C} 5-\mathrm{C} 6$ & $1.4015(15)$ \\
\hline $\mathrm{O} 1-\mathrm{H} 1 \mathrm{O}$ & $0.865(18)$ & $\mathrm{C} 5-\mathrm{H} 5$ & 0.9500 \\
\hline $\mathrm{O} 2-\mathrm{C} 7$ & $1.2716(13)$ & $\mathrm{C} 6-\mathrm{C} 7$ & $1.4862(14)$ \\
\hline $\mathrm{O} 2-\mathrm{Ca}^{\mathrm{ii}}$ & $2.6505(8)$ & $\mathrm{C} 7-\mathrm{Ca}^{\mathrm{ii}}$ & $2.9247(11)$ \\
\hline $\mathrm{O} 2-\mathrm{Sr}^{\mathrm{ii}}$ & $2.6505(8)$ & $\mathrm{C} 7-\mathrm{Sr}^{\mathrm{ii}}$ & $2.9247(11)$ \\
\hline $\mathrm{O} 1 \mathrm{~W}^{\mathrm{i}}-\mathrm{Sr} 1-\mathrm{O} 1 \mathrm{~W}$ & $104.89(5)$ & $\mathrm{O}^{\mathrm{iii}}-\mathrm{Sr} 1-\mathrm{Ca}^{\mathrm{ii}}$ & $114.28(2)$ \\
\hline $\mathrm{O} 1 \mathrm{~W}^{\mathrm{i}}-\mathrm{Sr} 1-\mathrm{O} 3$ & $73.82(3)$ & $\mathrm{O} 2^{\mathrm{ii}}-\mathrm{Sr} 1-\mathrm{Ca} 1^{\mathrm{ii}}$ & $83.294(17)$ \\
\hline $\mathrm{O} 1 \mathrm{~W}-\mathrm{Sr} 1-\mathrm{O} 3$ & $88.79(3)$ & $\mathrm{O} 2^{\mathrm{iii}}-\mathrm{Sr} 1-\mathrm{Ca}^{\mathrm{ii}}$ & $74.689(17)$ \\
\hline $\mathrm{O} 1 \mathrm{~W}^{\mathrm{i}}-\mathrm{Sr} 1-\mathrm{O}^{\mathrm{i}}$ & $88.79(3)$ & $\mathrm{C} 7^{\mathrm{ii}}-\mathrm{Sr} 1-\mathrm{Ca} 1^{\mathrm{ii}}$ & $59.55(2)$ \\
\hline $\mathrm{O} 1 \mathrm{~W}-\mathrm{Sr} 1-\mathrm{O}^{\mathrm{i}}$ & $73.82(3)$ & $\mathrm{C} 7^{\mathrm{iii}}-\mathrm{Sr} 1-\mathrm{Ca} 1^{\mathrm{ii}}$ & $92.57(2)$ \\
\hline $\mathrm{O} 3-\mathrm{Sr} 1-\mathrm{O} 3^{\mathrm{i}}$ & $151.53(4)$ & $\mathrm{Sr} 1^{\mathrm{ii}}-\mathrm{Sr} 1-\mathrm{Ca} 1^{\mathrm{ii}}$ & $0.000(7)$ \\
\hline $\mathrm{O} 1 \mathrm{~W}^{\mathrm{i}}-\mathrm{Sr} 1-\mathrm{O} 3^{\mathrm{ii}}$ & $88.84(3)$ & $\mathrm{O} 1 \mathrm{~W}^{\mathrm{i}}-\mathrm{Sr} 1-\mathrm{H} 2 \mathrm{~W}$ & $112.6(4)$ \\
\hline $\mathrm{O} 1 \mathrm{~W}-\mathrm{Sr} 1-\mathrm{O} 3^{\mathrm{ii}}$ & $151.47(3)$ & $\mathrm{O} 1 \mathrm{~W}-\mathrm{Sr} 1-\mathrm{H} 2 \mathrm{~W}$ & $16.0(4)$ \\
\hline $\mathrm{O} 3-\mathrm{Sr} 1-\mathrm{O}^{\mathrm{ii}}$ & $70.88(3)$ & $\mathrm{O} 3-\mathrm{Sr} 1-\mathrm{H} 2 \mathrm{~W}$ & $104.4(4)$ \\
\hline $\mathrm{O} 3^{\mathrm{i}}-\mathrm{Sr} 1-\mathrm{O} 3^{\mathrm{ii}}$ & $132.36(3)$ & $\mathrm{O} 3^{\mathrm{i}}-\mathrm{Sr} 1-\mathrm{H} 2 \mathrm{~W}$ & $61.3(4)$ \\
\hline $\mathrm{O} 1 \mathrm{~W}^{\mathrm{i}}-\mathrm{Sr} 1-\mathrm{O} 3^{\mathrm{iii}}$ & $151.47(3)$ & $\mathrm{O} 3^{\mathrm{ii}}-\mathrm{Sr} 1-\mathrm{H} 2 \mathrm{~W}$ & $156.3(4)$ \\
\hline $\mathrm{O} 1 \mathrm{~W}-\mathrm{Sr} 1-\mathrm{O} 3^{\mathrm{iii}}$ & $88.84(3)$ & $\mathrm{O}^{\mathrm{iii}}-\mathrm{Sr} 1-\mathrm{H} 2 \mathrm{~W}$ & $75.6(4)$ \\
\hline $\mathrm{O} 3-\mathrm{Sr} 1-\mathrm{O} 3^{\mathrm{iii}}$ & $132.36(3)$ & $\mathrm{O} 2{ }^{\mathrm{ii}}-\mathrm{Sr} 1-\mathrm{H} 2 \mathrm{~W}$ & $137.8(4)$ \\
\hline $\mathrm{O} 3^{\mathrm{i}}-\mathrm{Sr} 1-\mathrm{O} 3^{\mathrm{iii}}$ & $70.88(3)$ & $\mathrm{O} 2^{\mathrm{iii}}-\mathrm{Sr} 1-\mathrm{H} 2 \mathrm{~W}$ & $82.9(4)$ \\
\hline $\mathrm{O} 3^{\mathrm{ii}}-\mathrm{Sr} 1-\mathrm{O} 3^{\mathrm{iii}}$ & $90.48(4)$ & $\mathrm{C} 7{ }^{\mathrm{ii}}-\mathrm{Sr} 1-\mathrm{H} 2 \mathrm{~W}$ & $153.3(4)$ \\
\hline $\mathrm{O} 1 \mathrm{~W}^{\mathrm{i}}-\mathrm{Sr} 1-\mathrm{O} 2^{\mathrm{ii}}$ & $84.24(3)$ & $\mathrm{C}^{\mathrm{iii}}-\mathrm{Sr} 1-\mathrm{H} 2 \mathrm{~W}$ & $81.7(4)$ \\
\hline $\mathrm{O} 1 \mathrm{~W}-\mathrm{Sr} 1-\mathrm{O} 2^{\mathrm{ii}}$ & $153.57(3)$ & $\mathrm{Sr} 1^{\mathrm{ii}}-\mathrm{Sr} 1-\mathrm{H} 2 \mathrm{~W}$ & $136.2(4)$ \\
\hline $\mathrm{O} 3-\mathrm{Sr} 1-\mathrm{O} 2^{\mathrm{ii}}$ & $117.64(2)$ & $\mathrm{Ca} 1^{\mathrm{ii}}-\mathrm{Sr} 1-\mathrm{H} 2 \mathrm{~W}$ & $136.2(4)$ \\
\hline $\mathrm{O} 3^{\mathrm{i}}-\mathrm{Sr} 1-\mathrm{O} 2^{\mathrm{ii}}$ & $81.77(2)$ & $\mathrm{C} 1-\mathrm{O} 1-\mathrm{H} 1 \mathrm{O}$ & $108.5(12)$ \\
\hline $\mathrm{O} 3^{\mathrm{ii}}-\mathrm{Sr} 1-\mathrm{O} 2^{\mathrm{ii}}$ & $50.66(2)$ & $\mathrm{C} 7-\mathrm{O} 2-\mathrm{Ca} 1^{\mathrm{ii}}$ & $89.25(6)$ \\
\hline $\mathrm{O} 3^{\mathrm{iii}}-\mathrm{Sr} 1-\mathrm{O} 2^{\mathrm{ii}}$ & $73.47(3)$ & $\mathrm{C} 7-\mathrm{O} 2-\mathrm{Sr}^{\mathrm{ii}}$ & $89.25(6)$ \\
\hline $\mathrm{O} 1 \mathrm{~W}^{\mathrm{i}}-\mathrm{Sr} 1-\mathrm{O} 2^{\mathrm{iii}}$ & $153.57(3)$ & $\mathrm{Ca} 1^{\mathrm{ii}}-\mathrm{O} 2-\mathrm{Sr} 1^{\mathrm{ii}}$ & 0.0 \\
\hline $\mathrm{O} 1 \mathrm{~W}-\mathrm{Sr} 1-\mathrm{O} 2^{\mathrm{iii}}$ & $84.24(3)$ & $\mathrm{C} 7-\mathrm{O} 3-\mathrm{Sr} 1$ & $150.49(7)$ \\
\hline $\mathrm{O} 3-\mathrm{Sr} 1-\mathrm{O} 2^{\mathrm{iii}}$ & $81.77(2)$ & $\mathrm{C} 7-\mathrm{O} 3-\mathrm{Ca} 1^{\mathrm{ii}}$ & $96.62(6)$ \\
\hline $\mathrm{O} 3^{\mathrm{i}}-\mathrm{Sr} 1-\mathrm{O} 2^{\mathrm{iii}}$ & $117.64(2)$ & $\mathrm{Sr} 1-\mathrm{O} 3-\mathrm{Ca} 1^{\mathrm{ii}}$ & 109.1 \\
\hline $\mathrm{O} 3^{\mathrm{ii}}-\mathrm{Sr} 1-\mathrm{O} 2^{\mathrm{iii}}$ & $73.47(3)$ & $\mathrm{C} 7-\mathrm{O} 3-\mathrm{Sr} 1^{\mathrm{ii}}$ & $96.62(6)$ \\
\hline $\mathrm{O} 3^{\mathrm{iii}}-\mathrm{Sr} 1-\mathrm{O} 2^{\mathrm{iii}}$ & $50.66(2)$ & $\mathrm{Sr} 1-\mathrm{O} 3-\mathrm{Sr} 1^{\mathrm{ii}}$ & $109.12(3)$ \\
\hline
\end{tabular}




\begin{tabular}{|c|c|c|c|}
\hline $\mathrm{O} 2^{\mathrm{ii}}-\mathrm{Sr} 1-\mathrm{O} 2^{\mathrm{iii}}$ & $98.58(3)$ & $\mathrm{Ca} 1^{\mathrm{ii}}-\mathrm{O} 3-\mathrm{Sr} 1^{\mathrm{ii}}$ & 0.0 \\
\hline $\mathrm{O} 1 \mathrm{~W}^{\mathrm{i}}-\mathrm{Sr} 1-\mathrm{C}^{\mathrm{ii}}$ & $89.84(3)$ & $\mathrm{Sr} 1-\mathrm{O} 1 \mathrm{~W}-\mathrm{H} 1 \mathrm{~W}$ & $136.1(12)$ \\
\hline $\mathrm{O} 1 \mathrm{~W}-\mathrm{Sr} 1-\mathrm{C}^{\mathrm{ii}}$ & $165.27(3)$ & $\mathrm{Sr} 1-\mathrm{O} 1 \mathrm{~W}-\mathrm{H} 2 \mathrm{~W}$ & $112.0(12)$ \\
\hline $\mathrm{O} 3-\mathrm{Sr} 1-\mathrm{C}^{\mathrm{ii}}$ & $95.51(3)$ & $\mathrm{H} 1 \mathrm{~W}-\mathrm{O} 1 \mathrm{~W}-\mathrm{H} 2 \mathrm{~W}$ & $105.8(16)$ \\
\hline $\mathrm{O} 3^{\mathrm{i}}-\mathrm{Sr} 1-\mathrm{C}^{\mathrm{ii}}$ & $107.04(3)$ & $\mathrm{O} 1-\mathrm{C} 1-\mathrm{C} 2$ & $117.60(10)$ \\
\hline $\mathrm{O} 3^{\mathrm{ii}}-\mathrm{Sr} 1-\mathrm{C} 7^{\mathrm{ii}}$ & $25.39(3)$ & $\mathrm{O} 1-\mathrm{C} 1-\mathrm{C} 6$ & $122.00(9)$ \\
\hline 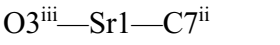 & $77.84(3)$ & $\mathrm{C} 2-\mathrm{C} 1-\mathrm{C} 6$ & $120.40(10)$ \\
\hline $\mathrm{O} 2^{\mathrm{ii}}-\mathrm{Sr} 1-\mathrm{C}^{\mathrm{ii}}$ & $25.77(3)$ & $\mathrm{C} 3-\mathrm{C} 2-\mathrm{C} 1$ & $119.46(10)$ \\
\hline $\mathrm{O} 2^{\mathrm{iii}}-\mathrm{Sr} 1-\mathrm{C} 7^{\mathrm{ii}}$ & $82.45(3)$ & $\mathrm{C} 3-\mathrm{C} 2-\mathrm{H} 2$ & 120.3 \\
\hline $\mathrm{O} 1 \mathrm{~W}^{\mathrm{i}}-\mathrm{Sr} 1-\mathrm{C} 7^{\mathrm{iii}}$ & $165.27(3)$ & $\mathrm{C} 1-\mathrm{C} 2-\mathrm{H} 2$ & 120.3 \\
\hline $\mathrm{O} 1 \mathrm{~W}-\mathrm{Sr} 1-\mathrm{C}^{\mathrm{iii}}$ & $89.84(3)$ & $\mathrm{C} 2-\mathrm{C} 3-\mathrm{C} 4$ & $121.07(10)$ \\
\hline $\mathrm{O} 3-\mathrm{Sr} 1-\mathrm{C} 7^{\mathrm{iii}}$ & $107.04(3)$ & $\mathrm{C} 2-\mathrm{C} 3-\mathrm{H} 3$ & 119.5 \\
\hline $\mathrm{O}^{\mathrm{i}}-\mathrm{Sr} 1-\mathrm{C} 7^{\mathrm{iii}}$ & $95.51(3)$ & $\mathrm{C} 4-\mathrm{C} 3-\mathrm{H} 3$ & 119.5 \\
\hline $\mathrm{O}^{\mathrm{ii}}-\mathrm{Sr} 1-\mathrm{C}^{\mathrm{iii}}$ & $77.84(3)$ & $\mathrm{C} 5-\mathrm{C} 4-\mathrm{C} 3$ & $119.40(11)$ \\
\hline $\mathrm{O} 3^{\mathrm{iii}}-\mathrm{Sr} 1-\mathrm{C} 7^{\mathrm{iii}}$ & $25.39(3)$ & $\mathrm{C} 5-\mathrm{C} 4-\mathrm{H} 4$ & 120.3 \\
\hline $\mathrm{O} 2^{\mathrm{ii}}-\mathrm{Sr} 1-\mathrm{C} 7^{\mathrm{iii}}$ & $82.45(3)$ & $\mathrm{C} 3-\mathrm{C} 4-\mathrm{H} 4$ & 120.3 \\
\hline $\mathrm{O} 2^{\mathrm{iii}}-\mathrm{Sr} 1-\mathrm{C}^{\mathrm{iii}}$ & $25.77(3)$ & $\mathrm{C} 4-\mathrm{C} 5-\mathrm{C} 6$ & $120.63(10)$ \\
\hline $\mathrm{C} 7^{\mathrm{ii}}-\mathrm{Sr} 1-\mathrm{C} 7^{\mathrm{iii}}$ & $75.42(4)$ & $\mathrm{C} 4-\mathrm{C} 5-\mathrm{H} 5$ & 119.7 \\
\hline $\mathrm{O} 1 \mathrm{~W}^{\mathrm{i}}-\mathrm{Sr} 1-\mathrm{Sr}^{\mathrm{ii}}$ & $79.60(2)$ & $\mathrm{C} 6-\mathrm{C} 5-\mathrm{H} 5$ & 119.7 \\
\hline $\mathrm{O} 1 \mathrm{~W}-\mathrm{Sr} 1-\mathrm{Sr} 1^{\mathrm{ii}}$ & $122.44(2)$ & $\mathrm{C} 1-\mathrm{C} 6-\mathrm{C} 5$ & $118.95(10)$ \\
\hline $\mathrm{O} 3-\mathrm{Sr} 1-\mathrm{Sr} 1^{\mathrm{ii}}$ & $36.194(18)$ & $\mathrm{C} 1-\mathrm{C} 6-\mathrm{C} 7$ & $120.66(9)$ \\
\hline $\mathrm{O}^{\mathrm{i}}-\mathrm{Sr} 1-\mathrm{Sr} 1^{\mathrm{ii}}$ & $161.916(19)$ & $\mathrm{C} 5-\mathrm{C} 6-\mathrm{C} 7$ & $120.39(9)$ \\
\hline $\mathrm{O} 3^{\mathrm{ii}}-\mathrm{Sr} 1-\mathrm{Sr}^{\mathrm{ii}}$ & $34.688(17)$ & $\mathrm{O} 3-\mathrm{C} 7-\mathrm{O} 2$ & $121.11(10)$ \\
\hline $\mathrm{O} 3^{\mathrm{iii}}-\mathrm{Sr} 1-\mathrm{Sr}^{\mathrm{ii}}$ & $114.28(2)$ & $\mathrm{O} 3-\mathrm{C} 7-\mathrm{C} 6$ & $119.79(9)$ \\
\hline $\mathrm{O} 2^{\mathrm{ii}}-\mathrm{Sr} 1-\mathrm{Sr} 1^{\mathrm{ii}}$ & $83.294(17)$ & $\mathrm{O} 2-\mathrm{C} 7-\mathrm{C} 6$ & $119.09(9)$ \\
\hline $\mathrm{O} 2^{\mathrm{iii}}-\mathrm{Sr} 1-\mathrm{Sr} 1^{\mathrm{ii}}$ & $74.689(17)$ & $\mathrm{O} 3-\mathrm{C} 7-\mathrm{Ca}^{\mathrm{ii}}$ & $57.99(5)$ \\
\hline $\mathrm{C}^{\mathrm{ii}}-\mathrm{Sr} 1-\mathrm{Sr} 1^{\mathrm{ii}}$ & $59.55(2)$ & $\mathrm{O} 2-\mathrm{C} 7-\mathrm{Ca}^{\mathrm{ii}}$ & $64.98(5)$ \\
\hline $\mathrm{C}^{\mathrm{iii}}-\mathrm{Sr} 1-\mathrm{Sr}^{\mathrm{ii}}$ & $92.57(2)$ & $\mathrm{C} 6-\mathrm{C} 7-\mathrm{Ca} 1^{\mathrm{ii}}$ & $164.81(7)$ \\
\hline $\mathrm{O} 1 \mathrm{~W}^{\mathrm{i}}-\mathrm{Sr} 1-\mathrm{Ca}^{\mathrm{ii}}$ & $79.60(2)$ & $\mathrm{O} 3-\mathrm{C} 7-\mathrm{Sr} 1^{\mathrm{ii}}$ & $57.99(5)$ \\
\hline $\mathrm{O} 1 \mathrm{~W}-\mathrm{Sr} 1-\mathrm{Ca} 1^{\mathrm{ii}}$ & $122.44(2)$ & $\mathrm{O} 2-\mathrm{C} 7-\mathrm{Sr}^{\mathrm{ii}}$ & $64.98(5)$ \\
\hline $\mathrm{O} 3-\mathrm{Sr} 1-\mathrm{Ca} 1^{\mathrm{ii}}$ & $36.194(18)$ & $\mathrm{C} 6-\mathrm{C} 7-\mathrm{Sr}^{\mathrm{ii}}$ & $164.81(7)$ \\
\hline $\mathrm{O} 3^{\mathrm{i}}-\mathrm{Sr} 1-\mathrm{Ca} 1^{\mathrm{ii}}$ & $161.916(19)$ & 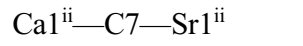 & 0.0 \\
\hline $\mathrm{O} 3^{\mathrm{ii}}-\mathrm{Sr} 1-\mathrm{Ca}^{\mathrm{ii}}$ & $34.688(17)$ & & \\
\hline $\mathrm{O} 1-\mathrm{C} 1-\mathrm{C} 2-\mathrm{C} 3$ & $-177.19(10)$ & $\mathrm{Sr}^{\mathrm{ii}}-\mathrm{O} 3-\mathrm{C} 7-\mathrm{Ca}^{\mathrm{ii}}$ & 0.0 \\
\hline $\mathrm{C} 6-\mathrm{C} 1-\mathrm{C} 2-\mathrm{C} 3$ & $3.20(16)$ & $\mathrm{Sr} 1-\mathrm{O} 3-\mathrm{C} 7-\mathrm{Sr} 1^{\mathrm{ii}}$ & $-150.97(15)$ \\
\hline $\mathrm{C} 1-\mathrm{C} 2-\mathrm{C} 3-\mathrm{C} 4$ & $-1.38(17)$ & $\mathrm{Ca} 1^{\mathrm{ii}}-\mathrm{O} 3-\mathrm{C} 7-\mathrm{Sr}^{1 \mathrm{ii}}$ & 0.0 \\
\hline $\mathrm{C} 2-\mathrm{C} 3-\mathrm{C} 4-\mathrm{C} 5$ & $-1.13(18)$ & $\mathrm{Ca} 1^{\mathrm{ii}}-\mathrm{O} 2-\mathrm{C} 7-\mathrm{O} 3$ & $-15.25(10)$ \\
\hline $\mathrm{C} 3-\mathrm{C} 4-\mathrm{C} 5-\mathrm{C} 6$ & $1.85(17)$ & $\mathrm{Sr} 1^{\mathrm{ii}}-\mathrm{O} 2-\mathrm{C} 7-\mathrm{O} 3$ & $-15.25(10)$ \\
\hline $\mathrm{O} 1-\mathrm{C} 1-\mathrm{C} 6-\mathrm{C} 5$ & $177.92(10)$ & $\mathrm{Ca} 1 \stackrel{\mathrm{ii}}{-\mathrm{O} 2}-\mathrm{C} 7-\mathrm{C} 6$ & $163.56(8)$ \\
\hline $\mathrm{C} 2-\mathrm{C} 1-\mathrm{C} 6-\mathrm{C} 5$ & $-2.49(15)$ & $\mathrm{Sr} 1^{\mathrm{ii}}-\mathrm{O} 2-\mathrm{C} 7-\mathrm{C} 6$ & $163.56(8)$ \\
\hline $\mathrm{O} 1-\mathrm{C} 1-\mathrm{C} 6-\mathrm{C} 7$ & $-1.84(15)$ & $\mathrm{Sr} 1^{\mathrm{ii}}-\mathrm{O} 2-\mathrm{C} 7-\mathrm{Ca} 1^{\mathrm{ii}}$ & 0.0 \\
\hline $\mathrm{C} 2-\mathrm{C} 1-\mathrm{C} 6-\mathrm{C} 7$ & $177.75(9)$ & $\mathrm{Ca} 1^{\mathrm{ii}}-\mathrm{O} 2-\mathrm{C} 7-\mathrm{Sr}^{\mathrm{ii}}$ & 0.0 \\
\hline $\mathrm{C} 4-\mathrm{C} 5-\mathrm{C} 6-\mathrm{C} 1$ & $-0.05(16)$ & $\mathrm{C} 1-\mathrm{C} 6-\mathrm{C} 7-\mathrm{O} 3$ & $-159.75(10)$ \\
\hline $\mathrm{C} 4-\mathrm{C} 5-\mathrm{C} 6-\mathrm{C} 7$ & $179.71(10)$ & $\mathrm{C} 5-\mathrm{C} 6-\mathrm{C} 7-\mathrm{O} 3$ & $20.50(15)$ \\
\hline $\mathrm{Sr} 1-\mathrm{O} 3-\mathrm{C} 7-\mathrm{O} 2$ & $-134.65(12)$ & $\mathrm{C} 1-\mathrm{C} 6-\mathrm{C} 7-\mathrm{O} 2$ & $21.43(15)$ \\
\hline $\mathrm{Ca} 1^{\mathrm{ii}}-\mathrm{O} 3-\mathrm{C} 7-\mathrm{O} 2$ & $16.32(11)$ & $\mathrm{C} 5-\mathrm{C} 6-\mathrm{C} 7-\mathrm{O} 2$ & $-158.32(10)$ \\
\hline $\mathrm{Sr}^{\mathrm{ii}}-\mathrm{O} 3-\mathrm{C} 7-\mathrm{O} 2$ & $16.32(11)$ & $\mathrm{C} 1-\mathrm{C} 6-\mathrm{C} 7-\mathrm{Ca}^{\mathrm{ii}}$ & $123.1(3)$ \\
\hline $\mathrm{Sr} 1-\mathrm{O} 3-\mathrm{C} 7-\mathrm{C} 6$ & $46.55(19)$ & $\mathrm{C} 5-\mathrm{C} 6-\mathrm{C} 7-\mathrm{Ca}^{\mathrm{ii}}$ & $-56.6(3)$ \\
\hline 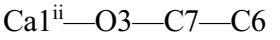 & $-162.47(8)$ & $\mathrm{C} 1-\mathrm{C} 6-\mathrm{C} 7-\mathrm{Sr}^{\mathrm{ii}}$ & $123.1(3)$ \\
\hline
\end{tabular}




\begin{tabular}{|c|c|c|c|}
\hline $\mathrm{Sr} 1 \mathrm{ii}-\mathrm{O} 3-\mathrm{C} 7-\mathrm{C} 6$ & $-162.47(8)$ & $\mathrm{C} 5-\mathrm{C} 6-\mathrm{C} 7-\mathrm{Sr} 1^{\mathrm{ii}}$ & $-56.6(3)$ \\
\hline $\mathrm{Sr} 1-\mathrm{O} 3-\mathrm{C} 7-\mathrm{Ca}^{\mathrm{ii}}$ & $-150.97(15)$ & & \\
\hline
\end{tabular}

Symmetry codes: (i) $-x+1, y,-z-1 / 2$; (ii) $-x+1,-y+1,-z$; (iii) $x,-y+1, z-1 / 2$.

Hydrogen-bond geometry $\left(A,{ }^{\circ}\right)$

\begin{tabular}{lllll}
\hline$D-\mathrm{H} \cdots A$ & $D-\mathrm{H}$ & $\mathrm{H} \cdots A$ & $D \cdots A$ & $D-\mathrm{H} \cdots A$ \\
\hline $\mathrm{O} 1-\mathrm{H} 1 O \cdots \mathrm{O} 2$ & $0.865(18)$ & $1.857(18)$ & $2.6165(11)$ & $145.5(16)$ \\
$\mathrm{O} 1 W-\mathrm{H} 1 W \cdots \mathrm{O} 1^{\text {iv }}$ & $0.85(2)$ & $2.00(2)$ & $2.8350(11)$ & $169.7(17)$ \\
$\mathrm{O} 1 W-\mathrm{H} 2 W \cdots \mathrm{O} 2^{\mathrm{v}}$ & $0.836(19)$ & $2.06(2)$ & $2.8849(12)$ & $166.8(17)$ \\
\hline
\end{tabular}

Symmetry codes: (iv) $-x+1 / 2,-y+1 / 2,-z ;$ (v) $x, y, z-1$.

\section{(CaSr7030)}

\section{Crystal data}

$\mathrm{C}_{14} \mathrm{H}_{14} \mathrm{Ca}_{0.83} \mathrm{O}_{8} \mathrm{Sr}_{0.17}$

$M_{r}=358.18$

Monoclinic, $C 2 / c$

$a=16.4921(10) \AA$

$b=11.5345(8) \AA$

$c=7.6679(5) \AA$

$\beta=91.838(6)^{\circ}$

$V=1457.90(16) \AA^{3}$

$Z=4$

\section{Data collection}

Oxford Diffraction Xcalibur E diffractometer

Radiation source: sealed tube $\omega$ scans
$F(000)=740$

$D_{\mathrm{x}}=1.632 \mathrm{Mg} \mathrm{m}^{-3}$

Mo $K \alpha$ radiation, $\lambda=0.71073 \AA$

Cell parameters from 2211 reflections

$\theta=3.4-30.1^{\circ}$

$\mu=1.01 \mathrm{~mm}^{-1}$

$T=150 \mathrm{~K}$

Cut prism, colourless

$0.26 \times 0.22 \times 0.12 \mathrm{~mm}$

\section{Refinement}

Refinement on $F^{2}$

Least-squares matrix: full

$R\left[F^{2}>2 \sigma\left(F^{2}\right)\right]=0.036$

$w R\left(F^{2}\right)=0.093$

$S=1.09$

1659 reflections

118 parameters

0 restraints
Absorption correction: multi-scan

CrysAlis PRO, Oxford Diffraction Ltd., Version

1.171.34.40 (release 27-08-2010 CrysAlis171 .NET)

(compiled Aug 27 2010,11:50:40) Empirical

absorption correction using spherical harmonics, implemented in SCALE3 ABSPACK scaling algorithm.

$T_{\min }=0.896, T_{\max }=1.000$

3241 measured reflections

1659 independent reflections

1494 reflections with $I>2 \sigma(I)$

$R_{\text {int }}=0.032$

$\theta_{\max }=27.5^{\circ}, \theta_{\min }=3.4^{\circ}$

$h=-21 \rightarrow 18$

$k=-12 \rightarrow 14$

$l=-9 \rightarrow 9$

Hydrogen site location: mixed

$\mathrm{H}$ atoms treated by a mixture of independent and constrained refinement

$w=1 /\left[\sigma^{2}\left(F_{\mathrm{o}}^{2}\right)+(0.0457 P)^{2}\right]$

where $P=\left(F_{\mathrm{o}}^{2}+2 F_{\mathrm{c}}^{2}\right) / 3$

$(\Delta / \sigma)_{\max }<0.001$

$\Delta \rho_{\max }=0.37 \mathrm{e} \AA^{-3}$

$\Delta \rho_{\min }=-0.38$ e $\AA^{-3}$ 


\section{Special details}

Geometry. All e.s.d.'s (except the e.s.d. in the dihedral angle between two 1.s. planes) are estimated using the full covariance matrix. The cell e.s.d.'s are taken into account individually in the estimation of e.s.d.'s in distances, angles and torsion angles; correlations between e.s.d.'s in cell parameters are only used when they are defined by crystal symmetry. An approximate (isotropic) treatment of cell e.s.d.'s is used for estimating e.s.d.'s involving l.s. planes.

Fractional atomic coordinates and isotropic or equivalent isotropic displacement parameters $\left(\AA^{2}\right)$

\begin{tabular}{llllll}
\hline & $x$ & $y$ & $z$ & $U_{\text {iso }} / U_{\text {eq }}$ & Occ. $(<1)$ \\
\hline Sr1 & 0.5000 & $0.44817(4)$ & -0.2500 & $0.01289(17)$ & $0.165(3)$ \\
Ca1 & 0.5000 & $0.44817(4)$ & -0.2500 & $0.01289(17)$ & $0.835(3)$ \\
O1 & $0.25637(7)$ & $0.25373(13)$ & $0.26791(18)$ & $0.0219(3)$ & $0.0196(3)$ \\
O2 & $0.37813(7)$ & $0.39984(12)$ & $0.26805(17)$ & $0.0205(3)$ & $0.0264(4)$ \\
O3 & $0.45477(7)$ & $0.39682(12)$ & $0.03736(17)$ & $0.0176(4)$ & $0.0217(4)$ \\
O1W & $0.39825(8)$ & $0.32205(14)$ & $-0.3747(2)$ & $0.026^{*}$ \\
C1 & $0.30155(10)$ & $0.18853(17)$ & $0.1580(2)$ & $0.0238(5)$ & $0.029^{*}$ \\
C2 & $0.27293(11)$ & $0.07830(19)$ & $0.1139(2)$ & $0.0248(5)$ & $0.030^{*}$ \\
H2 & 0.2225 & 0.0520 & 0.1553 & $0.0203(4)$ \\
C3 & $0.31847(12)$ & $0.00760(18)$ & $0.0095(3)$ & $0.024^{*}$ \\
H3 & 0.2993 & -0.0680 & -0.0189 & $0.0162(4)$ \\
C4 & $0.39199(13)$ & $0.04538(18)$ & $-0.0547(3)$ & $0.0158(4)$ \\
H4 & 0.4233 & -0.0044 & -0.1247 & $0.056(9)^{*}$ \\
C5 & $0.41889(11)$ & $0.15622(17)$ & $-0.0154(2)$ & $0.046(8)^{*}$ \\
H5 & 0.4683 & 0.1830 & -0.0615 & $0.046(8)^{*}$ \\
C6 & $0.37431(10)$ & $0.22961(17)$ & $0.0915(2)$ & \\
C7 & $0.40362(10)$ & $0.34874(16)$ & $0.1336(2)$ & \\
H1O & $0.2801(18)$ & $0.321(3)$ & $0.280(4)$ & $-0.331(3)$ & \\
H1W & $0.3554(16)$ & $0.290(2)$ & $-0.486(4)$ & & \\
H2W & $0.3898(16)$ & $0.329(2)$ & & & \\
& & & & &
\end{tabular}

Atomic displacement parameters $\left(\AA^{2}\right)$

\begin{tabular}{lllllll}
\hline & $U^{11}$ & $U^{22}$ & $U^{33}$ & $U^{12}$ & $U^{13}$ & $U^{23}$ \\
\hline Sr1 & $0.0097(2)$ & $0.0165(3)$ & $0.0126(2)$ & 0.000 & $0.00114(15)$ & 0.000 \\
Ca1 & $0.0097(2)$ & $0.0165(3)$ & $0.0126(2)$ & 0.000 & $0.00114(15)$ & 0.000 \\
O1 & $0.0170(6)$ & $0.0246(8)$ & $0.0244(7)$ & $-0.0032(6)$ & $0.0064(5)$ & $-0.0028(6)$ \\
O2 & $0.0178(6)$ & $0.0214(8)$ & $0.0196(7)$ & $-0.0031(6)$ & $0.0032(5)$ & $-0.0026(6)$ \\
O3 & $0.0155(6)$ & $0.0224(8)$ & $0.0238(7)$ & $-0.0032(5)$ & $0.0042(5)$ & $0.0006(6)$ \\
O1W & $0.0192(7)$ & $0.0375(9)$ & $0.0226(8)$ & $-0.0068(7)$ & $0.0032(6)$ & $0.0037(7)$ \\
C1 & $0.0160(8)$ & $0.0213(10)$ & $0.0153(8)$ & $0.0006(8)$ & $-0.0014(7)$ & $0.0019(8)$ \\
C2 & $0.0202(9)$ & $0.0258(10)$ & $0.0190(9)$ & $-0.0072(8)$ & $-0.0010(7)$ & $0.0032(9)$ \\
C3 & $0.0322(10)$ & $0.0177(10)$ & $0.0211(10)$ & $-0.0061(9)$ & $-0.0057(8)$ & $0.0012(9)$ \\
C4 & $0.0309(11)$ & $0.0229(11)$ & $0.0207(10)$ & $0.0027(8)$ & $0.0020(9)$ & $-0.0028(8)$ \\
C5 & $0.0189(8)$ & $0.0224(10)$ & $0.0197(9)$ & $-0.0002(8)$ & $0.0015(7)$ & $0.0004(8)$ \\
C6 & $0.0147(8)$ & $0.0185(9)$ & $0.0154(8)$ & $-0.0011(7)$ & $-0.0015(7)$ & $0.0006(8)$ \\
C7 & $0.0097(7)$ & $0.0191(9)$ & $0.0182(9)$ & $0.0020(7)$ & $-0.0029(7)$ & $0.0006(8)$ \\
\end{tabular}

Geometric parameters $\left(A,{ }^{\circ}\right)$

\begin{tabular}{lllr}
\hline $\mathrm{S} 1-\mathrm{O} 1 \mathrm{~W}$ & $2.3968(15)$ & $\mathrm{O} 3-\mathrm{C} 7$ & $1.266(2)$ \\
$\mathrm{Sr} 1-\mathrm{O} 1 W^{\mathrm{i}}$ & $2.3968(15)$ & $\mathrm{O} 3-\mathrm{Ca} 1^{\mathrm{ii}}$ & $2.5166(14)$ \\
$\mathrm{Sr} 1-\mathrm{O} 3$ & $2.4224(13)$ & $\mathrm{O} 3-\mathrm{Sr} 1^{\mathrm{ii}}$ & $2.5166(14)$
\end{tabular}




\begin{tabular}{|c|c|c|c|}
\hline $\mathrm{Sr} 1-\mathrm{O} 3^{\mathrm{i}}$ & $2.4224(13)$ & $\mathrm{O} 1 \mathrm{~W}-\mathrm{H} 1 \mathrm{~W}$ & $0.87(3)$ \\
\hline $\mathrm{Sr} 1-\mathrm{O} 3^{\mathrm{ii}}$ & $2.5166(14)$ & $\mathrm{O} 1 \mathrm{~W}-\mathrm{H} 2 \mathrm{~W}$ & $0.86(3)$ \\
\hline $\mathrm{Sr} 1-\mathrm{O} 3^{\mathrm{iii}}$ & $2.5166(14)$ & $\mathrm{C} 1-\mathrm{C} 2$ & $1.394(3)$ \\
\hline $\mathrm{Sr} 1-\mathrm{O} 2^{\mathrm{ii}}$ & $2.6739(13)$ & $\mathrm{C} 1-\mathrm{C} 6$ & $1.401(2)$ \\
\hline $\mathrm{Sr} 1-\mathrm{O} 2^{\mathrm{iii}}$ & $2.6739(13)$ & $\mathrm{C} 2-\mathrm{C} 3$ & $1.381(3)$ \\
\hline $\mathrm{Sr} 1-\mathrm{C} 7^{\mathrm{ii}}$ & $2.9529(19)$ & $\mathrm{C} 2-\mathrm{H} 2$ & 0.9500 \\
\hline $\mathrm{Sr} 1-\mathrm{C}^{\mathrm{iii}}$ & $2.9529(19)$ & $\mathrm{C} 3-\mathrm{C} 4$ & $1.393(3)$ \\
\hline $\mathrm{Sr} 1-\mathrm{Sr} 1^{\mathrm{ii}}$ & $4.0160(4)$ & $\mathrm{C} 3-\mathrm{H} 3$ & 0.9500 \\
\hline $\mathrm{Sr} 1-\mathrm{Ca} 1^{\mathrm{ii}}$ & $4.0160(4)$ & $\mathrm{C} 4-\mathrm{C} 5$ & $1.383(3)$ \\
\hline $\mathrm{Sr} 1-\mathrm{H} 2 \mathrm{~W}$ & $2.87(3)$ & $\mathrm{C} 4-\mathrm{H} 4$ & 0.9500 \\
\hline $\mathrm{O} 1-\mathrm{C} 1$ & $1.368(2)$ & $\mathrm{C} 5-\mathrm{C} 6$ & $1.403(3)$ \\
\hline $\mathrm{O} 1-\mathrm{H} 1 \mathrm{O}$ & $0.87(3)$ & $\mathrm{C} 5-\mathrm{H} 5$ & 0.9500 \\
\hline $\mathrm{O} 2-\mathrm{C} 7$ & $1.271(2)$ & $\mathrm{C} 6-\mathrm{C} 7$ & $1.489(3)$ \\
\hline $\mathrm{O} 2-\mathrm{Ca}^{\mathrm{ii}}$ & $2.6740(13)$ & $\mathrm{C} 7-\mathrm{Ca} 1^{\mathrm{ii}}$ & $2.9529(19)$ \\
\hline $\mathrm{O} 2-\mathrm{Sr}^{1 \mathrm{ii}}$ & $2.6740(13)$ & $\mathrm{C} 7-\mathrm{Sr} 1^{\mathrm{ii}}$ & $2.9529(19)$ \\
\hline $\mathrm{O} 1 \mathrm{~W}-\mathrm{Sr} 1-\mathrm{O}^{\mathrm{i}}$ & $105.26(8)$ & $\mathrm{O} 3^{\mathrm{iii}}-\mathrm{Sr} 1-\mathrm{Ca} 1^{\mathrm{ii}}$ & $113.45(4)$ \\
\hline $\mathrm{O} 1 \mathrm{~W}-\mathrm{Sr} 1-\mathrm{O} 3$ & $89.00(5)$ & $\mathrm{O} 2^{\mathrm{ii}}-\mathrm{Sr} 1-\mathrm{Ca} 1^{\mathrm{ii}}$ & $82.95(3)$ \\
\hline $\mathrm{O} 1 \mathrm{~W}^{\mathrm{i}}-\mathrm{Sr} 1-\mathrm{O} 3$ & $73.78(5)$ & $\mathrm{O} 2^{\mathrm{iii}}-\mathrm{Sr} 1-\mathrm{Ca} 1^{\mathrm{ii}}$ & $74.48(3)$ \\
\hline $\mathrm{O} 1 \mathrm{~W}-\mathrm{Sr} 1-\mathrm{O}^{\mathrm{i}}$ & $73.78(5)$ & $\mathrm{C} 7^{\mathrm{ii}}-\mathrm{Sr} 1-\mathrm{Ca} 1^{\mathrm{ii}}$ & $59.45(4)$ \\
\hline $\mathrm{O} 1 \mathrm{~W}^{\mathrm{i}}-\mathrm{Sr} 1-\mathrm{O}^{\mathrm{i}}$ & $89.00(5)$ & $\mathrm{C}^{\mathrm{iii}}-\mathrm{Sr} 1-\mathrm{Ca} 1^{\mathrm{ii}}$ & $92.06(4)$ \\
\hline $\mathrm{O} 3-\mathrm{Sr} 1-\mathrm{O}^{\mathrm{i}}$ & $151.69(7)$ & $\mathrm{Sr} 1^{\mathrm{ii}}-\mathrm{Sr} 1-\mathrm{Ca} 1^{\mathrm{ii}}$ & 0.0 \\
\hline $\mathrm{O} 1 \mathrm{~W}-\mathrm{Sr} 1-\mathrm{O} 3^{\mathrm{ii}}$ & $151.52(4)$ & $\mathrm{O} 1 \mathrm{~W}-\mathrm{Sr} 1-\mathrm{H} 2 \mathrm{~W}$ & $15.7(6)$ \\
\hline $\mathrm{O} 1 \mathrm{~W}^{\mathrm{i}}-\mathrm{Sr} 1-\mathrm{O}^{\mathrm{ii}}$ & $89.05(5)$ & $\mathrm{O} 1 \mathrm{~W}^{\mathrm{i}}-\mathrm{Sr} 1-\mathrm{H} 2 \mathrm{~W}$ & $112.4(6)$ \\
\hline $\mathrm{O} 3-\mathrm{Sr} 1-\mathrm{O}^{\mathrm{ii}}$ & $71.21(5)$ & $\mathrm{O} 3-\mathrm{Sr} 1-\mathrm{H} 2 \mathrm{~W}$ & $104.4(6)$ \\
\hline $\mathrm{O} 3^{\mathrm{i}}-\mathrm{Sr} 1-\mathrm{O} 3^{\mathrm{ii}}$ & $132.03(5)$ & $\mathrm{O} 3{ }^{\mathrm{i}}-\mathrm{Sr} 1-\mathrm{H} 2 \mathrm{~W}$ & $61.2(5)$ \\
\hline $\mathrm{O} 1 \mathrm{~W}-\mathrm{Sr} 1-\mathrm{O} 3^{\mathrm{iii}}$ & $89.05(5)$ & $\mathrm{O} 3{ }^{\mathrm{ii}}-\mathrm{Sr} 1-\mathrm{H} 2 \mathrm{~W}$ & $156.4(6)$ \\
\hline $\mathrm{O} 1 \mathrm{~W}^{\mathrm{i}}-\mathrm{Sr} 1-\mathrm{O} 3^{\mathrm{iii}}$ & $151.52(5)$ & $\mathrm{O} 33^{\mathrm{iii}}-\mathrm{Sr} 1-\mathrm{H} 2 \mathrm{~W}$ & $76.4(6)$ \\
\hline $\mathrm{O} 3-\mathrm{Sr} 1-\mathrm{O} 3^{\mathrm{iii}}$ & $132.03(5)$ & $\mathrm{O} 2 \mathrm{ii}-\mathrm{Sr} 1-\mathrm{H} 2 \mathrm{~W}$ & $138.0(6)$ \\
\hline $\mathrm{O} 3^{\mathrm{i}}-\mathrm{Sr} 1-\mathrm{O} 3^{\mathrm{iii}}$ & $71.21(5)$ & $\mathrm{O} 22^{\mathrm{iii}}-\mathrm{Sr} 1-\mathrm{H} 2 \mathrm{~W}$ & $83.4(6)$ \\
\hline $\mathrm{O} 3^{\mathrm{ii}}-\mathrm{Sr} 1-\mathrm{O} 3^{\mathrm{iii}}$ & $89.46(7)$ & $\mathrm{C}^{\mathrm{ii}}-\mathrm{Sr} 1-\mathrm{H} 2 \mathrm{~W}$ & $153.4(6)$ \\
\hline $\mathrm{O} 1 \mathrm{~W}-\mathrm{Sr} 1-\mathrm{O} 2^{\mathrm{ii}}$ & $153.52(5)$ & $\mathrm{C} 7{ }^{\mathrm{iii}}-\mathrm{Sr} 1-\mathrm{H} 2 \mathrm{~W}$ & $82.3(6)$ \\
\hline $\mathrm{O} 1 \mathrm{~W}^{\mathrm{i}}-\mathrm{Sr} 1-\mathrm{O} 2^{\mathrm{ii}}$ & $84.31(5)$ & $\mathrm{Sr} 1{ }^{\mathrm{ii}}-\mathrm{Sr} 1-\mathrm{H} 2 \mathrm{~W}$ & $136.4(5)$ \\
\hline $\mathrm{O} 3-\mathrm{Sr} 1-\mathrm{O} 2^{\mathrm{ii}}$ & $117.47(4)$ & $\mathrm{Ca} 1^{\mathrm{ii}}-\mathrm{Sr} 1-\mathrm{H} 2 \mathrm{~W}$ & $136.4(5)$ \\
\hline $\mathrm{O} 3^{\mathrm{i}}-\mathrm{Sr} 1-\mathrm{O} 2^{\mathrm{ii}}$ & $81.91(4)$ & $\mathrm{C} 1-\mathrm{O} 1-\mathrm{H} 1 \mathrm{O}$ & $107.5(19)$ \\
\hline $\mathrm{O} 3^{\mathrm{ii}}-\mathrm{Sr} 1-\mathrm{O} 2^{\mathrm{ii}}$ & $50.21(4)$ & $\mathrm{C} 7-\mathrm{O} 2-\mathrm{Ca} 1^{\mathrm{ii}}$ & $89.62(10)$ \\
\hline $\mathrm{O} 3^{\mathrm{iii}}-\mathrm{Sr} 1-\mathrm{O} 2^{\mathrm{ii}}$ & $73.04(4)$ & $\mathrm{C} 7-\mathrm{O} 2-\mathrm{Sr} 1^{\mathrm{ii}}$ & $89.62(10)$ \\
\hline $\mathrm{O} 1 \mathrm{~W}-\mathrm{Sr} 1-\mathrm{O} 2^{\mathrm{iii}}$ & $84.31(5)$ & $\mathrm{Ca} 1^{\mathrm{ii}}-\mathrm{O} 2-\mathrm{Sr} 1^{\mathrm{ii}}$ & 0.0 \\
\hline $\mathrm{O} 1 \mathrm{~W}^{\mathrm{i}}-\mathrm{Sr} 1-\mathrm{O} 2^{\mathrm{iii}}$ & $153.52(5)$ & $\mathrm{C} 7-\mathrm{O} 3-\mathrm{Sr} 1$ & $150.16(12)$ \\
\hline $\mathrm{O} 3-\mathrm{Sr} 1-\mathrm{O} 2^{\mathrm{iii}}$ & $81.91(4)$ & $\mathrm{C} 7-\mathrm{O} 3-\mathrm{Ca} 1^{\mathrm{ii}}$ & $97.05(11)$ \\
\hline $\mathrm{O} 3^{\mathrm{i}}-\mathrm{Sr} 1-\mathrm{O} 2^{\mathrm{iii}}$ & $117.47(4)$ & $\mathrm{Sr} 1-\mathrm{O} 3-\mathrm{Ca}^{\mathrm{ii}}$ & 108.8 \\
\hline $\mathrm{O} 3^{\mathrm{ii}}-\mathrm{Sr} 1-\mathrm{O} 2^{\mathrm{iii}}$ & $73.04(4)$ & $\mathrm{C} 7-\mathrm{O} 3-\mathrm{Sr} 1^{\mathrm{ii}}$ & $97.05(11)$ \\
\hline $\mathrm{O} 3^{\mathrm{iii}}-\mathrm{Sr} 1-\mathrm{O} 2^{\mathrm{iii}}$ & $50.21(4)$ & $\mathrm{Sr} 1-\mathrm{O} 3-\mathrm{Sr}^{\mathrm{ii}}$ & $108.79(5)$ \\
\hline $\mathrm{O} 2^{\mathrm{ii}}-\mathrm{Sr} 1-\mathrm{O} 2^{\mathrm{iii}}$ & $98.07(6)$ & $\mathrm{Ca} 1^{\mathrm{ii}}-\mathrm{O} 3-\mathrm{Sr} 1^{\mathrm{ii}}$ & 0.0 \\
\hline $\mathrm{O} 1 \mathrm{~W}-\mathrm{Sr} 1-\mathrm{C}^{\mathrm{ii}}$ & $164.87(5)$ & $\mathrm{Sr} 1-\mathrm{O} 1 \mathrm{~W}-\mathrm{H} 1 \mathrm{~W}$ & $132.2(17)$ \\
\hline $\mathrm{O} 1 \mathrm{~W}^{\mathrm{i}}-\mathrm{Sr} 1-\mathrm{C}^{\mathrm{ii}}$ & $89.87(5)$ & $\mathrm{Sr} 1-\mathrm{O} 1 \mathrm{~W}-\mathrm{H} 2 \mathrm{~W}$ & $115.4(18)$ \\
\hline $\mathrm{O} 3-\mathrm{Sr} 1-\mathrm{C}^{\mathrm{ii}}$ & $95.59(5)$ & $\mathrm{H} 1 \mathrm{~W}-\mathrm{O} 1 \mathrm{~W}-\mathrm{H} 2 \mathrm{~W}$ & $108(2)$ \\
\hline $\mathrm{O} 3^{\mathrm{i}}-\mathrm{Sr} 1-\mathrm{C} 7^{\mathrm{ii}}$ & $106.89(5)$ & $\mathrm{O} 1-\mathrm{C} 1-\mathrm{C} 2$ & $117.64(16)$ \\
\hline $\mathrm{O} 3^{\mathrm{ii}}-\mathrm{Sr} 1-\mathrm{C}^{\mathrm{ii}}$ & $25.19(4)$ & $\mathrm{O} 1-\mathrm{C} 1-\mathrm{C} 6$ & $121.87(17)$ \\
\hline $\mathrm{O}^{\mathrm{iii}}-\mathrm{Sr} 1-\mathrm{C}^{\mathrm{ii}}$ & $77.16(5)$ & $\mathrm{C} 2-\mathrm{C} 1-\mathrm{C} 6$ & $120.48(18)$ \\
\hline $\mathrm{O} 2^{\mathrm{ii}}-\mathrm{Sr} 1-\mathrm{C} 7^{\mathrm{ii}}$ & $25.49(4)$ & $\mathrm{C} 3-\mathrm{C} 2-\mathrm{C} 1$ & $119.53(17)$ \\
\hline $\mathrm{O} 2^{\mathrm{iii}}-\mathrm{Sr} 1-\mathrm{C} 7^{\mathrm{ii}}$ & $82.09(5)$ & $\mathrm{C} 3-\mathrm{C} 2-\mathrm{H} 2$ & 120.2 \\
\hline
\end{tabular}




\begin{tabular}{|c|c|c|c|}
\hline $\mathrm{O} 1 \mathrm{~W}-\mathrm{Sr} 1-\mathrm{C} 7^{\mathrm{iii}}$ & $89.87(5)$ & $\mathrm{C} 1-\mathrm{C} 2-\mathrm{H} 2$ & 120.2 \\
\hline $\mathrm{O} 1 \mathrm{~W}^{\mathrm{i}}-\mathrm{Sr} 1-\mathrm{C} 7^{\mathrm{iii}}$ & $164.87(5)$ & $\mathrm{C} 2-\mathrm{C} 3-\mathrm{C} 4$ & $121.04(19)$ \\
\hline $\mathrm{O} 3-\mathrm{Sr} 1-\mathrm{C} 7^{\mathrm{iii}}$ & $106.89(5)$ & $\mathrm{C} 2-\mathrm{C} 3-\mathrm{H} 3$ & 119.5 \\
\hline $\mathrm{O} 3^{\mathrm{i}}-\mathrm{Sr} 1-\mathrm{C} 7^{\mathrm{iii}}$ & $95.60(5)$ & $\mathrm{C} 4-\mathrm{C} 3-\mathrm{H} 3$ & 119.5 \\
\hline $\mathrm{O} 3^{\mathrm{ii}}-\mathrm{Sr} 1-\mathrm{C} 7^{\mathrm{iii}}$ & $77.16(5)$ & $\mathrm{C} 5-\mathrm{C} 4-\mathrm{C} 3$ & $119.26(19)$ \\
\hline $\mathrm{O}{ }^{\mathrm{iii}}-\mathrm{Sr} 1-\mathrm{C}^{\mathrm{iii}}$ & $25.19(4)$ & $\mathrm{C} 5-\mathrm{C} 4-\mathrm{H} 4$ & 120.4 \\
\hline $\mathrm{O} 2^{\mathrm{ii}}-\mathrm{Sr} 1-\mathrm{C} 7^{\mathrm{iii}}$ & $82.09(5)$ & $\mathrm{C} 3-\mathrm{C} 4-\mathrm{H} 4$ & 120.4 \\
\hline $\mathrm{O} 2^{\mathrm{iii}}-\mathrm{Sr} 1-\mathrm{C} 7^{\mathrm{iii}}$ & $25.49(4)$ & $\mathrm{C} 4-\mathrm{C} 5-\mathrm{C} 6$ & $120.96(17)$ \\
\hline $\mathrm{C}^{\mathrm{ii}}-\mathrm{Sr} 1-\mathrm{C} 7^{\mathrm{iii}}$ & $75.01(7)$ & $\mathrm{C} 4-\mathrm{C} 5-\mathrm{H} 5$ & 119.5 \\
\hline $\mathrm{O} 1 \mathrm{~W}-\mathrm{Sr} 1-\mathrm{Sr} 1^{\mathrm{ii}}$ & $122.70(4)$ & $\mathrm{C} 6-\mathrm{C} 5-\mathrm{H} 5$ & 119.5 \\
\hline $\mathrm{O} 1 \mathrm{~W}^{\mathrm{i}}-\mathrm{Sr} 1-\mathrm{Sr} 1^{\mathrm{ii}}$ & $79.70(4)$ & $\mathrm{C} 1-\mathrm{C} 6-\mathrm{C} 5$ & $118.66(17)$ \\
\hline $\mathrm{O} 3-\mathrm{Sr} 1-\mathrm{Sr} 1^{\mathrm{ii}}$ & $36.39(3)$ & $\mathrm{C} 1-\mathrm{C} 6-\mathrm{C} 7$ & $120.61(16)$ \\
\hline $\mathrm{O}^{\mathrm{i}}-\mathrm{Sr} 1-\mathrm{Sr} 1^{\mathrm{ii}}$ & $161.92(3)$ & $\mathrm{C} 5-\mathrm{C} 6-\mathrm{C} 7$ & $120.73(16)$ \\
\hline $\mathrm{O} 3^{\mathrm{ii}}-\mathrm{Sr} 1-\mathrm{Sr} 1^{\mathrm{ii}}$ & $34.82(3)$ & $\mathrm{O} 3-\mathrm{C} 7-\mathrm{O} 2$ & $120.89(17)$ \\
\hline $\mathrm{O} 3^{\mathrm{iii}}-\mathrm{Sr} 1-\mathrm{Sr}^{\mathrm{ii}}$ & $113.45(4)$ & $\mathrm{O} 3-\mathrm{C} 7-\mathrm{C} 6$ & $119.73(16)$ \\
\hline $\mathrm{O} 2^{\mathrm{ii}}-\mathrm{Sr} 1-\mathrm{Sr} 1^{\mathrm{ii}}$ & $82.95(3)$ & $\mathrm{O} 2-\mathrm{C} 7-\mathrm{C} 6$ & $119.36(15)$ \\
\hline $\mathrm{O} 2^{\mathrm{iii}}-\mathrm{Sr} 1-\mathrm{Sr} 1^{\mathrm{ii}}$ & $74.48(3)$ & $\mathrm{O} 3-\mathrm{C} 7-\mathrm{Ca}^{\mathrm{ii}}$ & $57.76(9)$ \\
\hline $\mathrm{C}^{\mathrm{ii}}-\mathrm{Sr} 1-\mathrm{Sr}^{\mathrm{ii}}$ & $59.45(4)$ & $\mathrm{O} 2-\mathrm{C} 7-\mathrm{Ca} 1^{\mathrm{ii}}$ & $64.89(9)$ \\
\hline $\mathrm{C} 7^{\mathrm{iii}}-\mathrm{Sr} 1-\mathrm{Sr} 1^{\mathrm{ii}}$ & $92.06(4)$ & $\mathrm{C} 6-\mathrm{C} 7-\mathrm{Ca} 1^{\mathrm{ii}}$ & $164.95(11)$ \\
\hline $\mathrm{O} 1 \mathrm{~W}-\mathrm{Sr} 1-\mathrm{Ca} 1^{\mathrm{ii}}$ & $122.70(4)$ & $\mathrm{O} 3-\mathrm{C} 7-\mathrm{Sr}^{\mathrm{ii}}$ & $57.76(9)$ \\
\hline $\mathrm{O} 1 \mathrm{~W}^{\mathrm{i}}-\mathrm{Sr} 1-\mathrm{Ca} 1^{\mathrm{ii}}$ & $79.70(4)$ & $\mathrm{O} 2-\mathrm{C} 7-\mathrm{Sr}^{\mathrm{ii}}$ & $64.89(9)$ \\
\hline $\mathrm{O} 3-\mathrm{Sr} 1-\mathrm{Ca} 1^{\mathrm{ii}}$ & $36.39(3)$ & $\mathrm{C} 6-\mathrm{C} 7-\mathrm{Sr}^{\mathrm{ii}}$ & $164.95(11)$ \\
\hline $\mathrm{O} 3^{\mathrm{i}}-\mathrm{Sr} 1-\mathrm{Ca} 1^{\mathrm{ii}}$ & $161.92(3)$ & $\mathrm{Ca} 1^{\mathrm{ii}}-\mathrm{C} 7-\mathrm{Sr}^{\mathrm{ii}}$ & 0.0 \\
\hline $\mathrm{O} 3^{\mathrm{ii}}-\mathrm{Sr} 1-\mathrm{Ca} 1^{\mathrm{ii}}$ & $34.82(3)$ & & \\
\hline $\mathrm{O} 1-\mathrm{C} 1-\mathrm{C} 2-\mathrm{C} 3$ & $-177.15(17)$ & $\mathrm{Sr}^{\mathrm{ii}}-\mathrm{O} 3-\mathrm{C} 7-\mathrm{Ca} 1^{\mathrm{ii}}$ & 0.0 \\
\hline $\mathrm{C} 6-\mathrm{C} 1-\mathrm{C} 2-\mathrm{C} 3$ & $2.6(3)$ & $\mathrm{Sr} 1-\mathrm{O} 3-\mathrm{C} 7-\mathrm{Sr} 1^{\mathrm{ii}}$ & $-150.2(3)$ \\
\hline $\mathrm{C} 1-\mathrm{C} 2-\mathrm{C} 3-\mathrm{C} 4$ & $-1.0(3)$ & $\mathrm{Ca} 1^{\mathrm{ii}}-\mathrm{O} 3-\mathrm{C} 7-\mathrm{Sr} 1^{\mathrm{ii}}$ & 0.0 \\
\hline $\mathrm{C} 2-\mathrm{C} 3-\mathrm{C} 4-\mathrm{C} 5$ & $-1.1(3)$ & $\mathrm{Ca} 1^{\mathrm{ii}}-\mathrm{O} 2-\mathrm{C} 7-\mathrm{O} 3$ & $-14.77(16)$ \\
\hline $\mathrm{C} 3-\mathrm{C} 4-\mathrm{C} 5-\mathrm{C} 6$ & $1.7(3)$ & 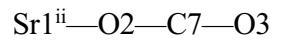 & $-14.77(16)$ \\
\hline $\mathrm{O} 1-\mathrm{C} 1-\mathrm{C} 6-\mathrm{C} 5$ & $177.71(16)$ & $\mathrm{Ca} 1{ }^{\mathrm{ii}}-\mathrm{O} 2-\mathrm{C} 7-\mathrm{C} 6$ & $163.75(14)$ \\
\hline $\mathrm{C} 2-\mathrm{C} 1-\mathrm{C} 6-\mathrm{C} 5$ & $-2.0(3)$ & 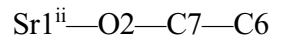 & $163.75(14)$ \\
\hline $\mathrm{O} 1-\mathrm{C} 1-\mathrm{C} 6-\mathrm{C} 7$ & $-2.3(3)$ & $\mathrm{Sr} 1^{\mathrm{ii}}-\mathrm{O} 2-\mathrm{C} 7-\mathrm{Ca} 1^{\mathrm{ii}}$ & 0.0 \\
\hline $\mathrm{C} 2-\mathrm{C} 1-\mathrm{C} 6-\mathrm{C} 7$ & $177.94(16)$ & $\mathrm{Ca} 1^{\mathrm{ii}}-\mathrm{O} 2-\mathrm{C} 7-\mathrm{Sr} 1^{\mathrm{ii}}$ & 0.0 \\
\hline $\mathrm{C} 4-\mathrm{C} 5-\mathrm{C} 6-\mathrm{C} 1$ & $-0.1(3)$ & $\mathrm{C} 1-\mathrm{C} 6-\mathrm{C} 7-\mathrm{O} 3$ & $-159.89(17)$ \\
\hline $\mathrm{C} 4-\mathrm{C} 5-\mathrm{C} 6-\mathrm{C} 7$ & $179.91(17)$ & $\mathrm{C} 5-\mathrm{C} 6-\mathrm{C} 7-\mathrm{O} 3$ & $20.1(3)$ \\
\hline $\mathrm{Sr} 1-\mathrm{O} 3-\mathrm{C} 7-\mathrm{O} 2$ & $-134.4(2)$ & $\mathrm{C} 1-\mathrm{C} 6-\mathrm{C} 7-\mathrm{O} 2$ & $21.6(3)$ \\
\hline 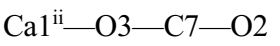 & $15.83(17)$ & $\mathrm{C} 5-\mathrm{C} 6-\mathrm{C} 7-\mathrm{O} 2$ & $-158.43(17)$ \\
\hline $\mathrm{Sr}^{\mathrm{ii}}-\mathrm{O} 3-\mathrm{C} 7-\mathrm{O} 2$ & $15.83(17)$ & $\mathrm{C} 1-\mathrm{C} 6-\mathrm{C} 7-\mathrm{Ca}^{\mathrm{ii}}$ & $124.1(4)$ \\
\hline $\mathrm{Sr} 1-\mathrm{O} 3-\mathrm{C} 7-\mathrm{C} 6$ & $47.1(3)$ & $\mathrm{C} 5-\mathrm{C} 6-\mathrm{C} 7-\mathrm{Ca}^{\mathrm{ii}}$ & $-55.9(5)$ \\
\hline 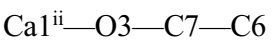 & $-162.68(13)$ & $\mathrm{C} 1-\mathrm{C} 6-\mathrm{C} 7-\mathrm{Sr}^{\mathrm{ii}}$ & $124.1(4)$ \\
\hline $\mathrm{Sr}^{\mathrm{ii}}-\mathrm{O} 3-\mathrm{C} 7-\mathrm{C} 6$ & $-162.68(13)$ & $\mathrm{C} 5-\mathrm{C} 6-\mathrm{C} 7-\mathrm{Sr}^{\mathrm{ii}}$ & $-55.9(5)$ \\
\hline $\mathrm{Sr} 1-\mathrm{O} 3-\mathrm{C} 7-\mathrm{Ca}^{\mathrm{ii}}$ & $-150.2(3)$ & & \\
\hline
\end{tabular}

Symmetry codes: (i) $-x+1, y,-z-1 / 2$; (ii) $-x+1,-y+1,-z$; (iii) $x,-y+1, z-1 / 2$.

Hydrogen-bond geometry $\left(A,{ }^{\circ}\right)$

\begin{tabular}{lllll}
\hline$D-\mathrm{H} \cdots A$ & $D-\mathrm{H}$ & $\mathrm{H} \cdots A$ & $D \cdots A$ & $D-\mathrm{H} \cdots A$ \\
\hline $\mathrm{O} 1-\mathrm{H} 1 O \cdots \mathrm{O} 2$ & $0.87(3)$ & $1.86(3)$ & $2.6216(18)$ & $145(3)$
\end{tabular}




\begin{tabular}{lllll}
$\mathrm{O} 1 W-\mathrm{H} 1 W \cdots \mathrm{O} 1^{\mathrm{iv}}$ & $0.87(3)$ & $1.99(3)$ & $2.8409(19)$ & $166(3)$ \\
$\mathrm{O} 1 W-\mathrm{H} 2 W \cdots \mathrm{O} 2^{\mathrm{v}}$ & $0.86(3)$ & $2.06(3)$ & $2.891(2)$ & $161(3)$ \\
\hline
\end{tabular}

Symmetry codes: (iv) $-x+1 / 2,-y+1 / 2,-z$; (v) $x, y, z-1$.

\section{(CaSr6040)}

\section{Crystal data}

$$
\begin{aligned}
& \mathrm{C}_{14} \mathrm{H}_{14} \mathrm{Ca}_{0.69} \mathrm{O}_{8} \mathrm{Sr}_{0.31} \\
& M_{r}=364.90 \\
& \text { Monoclinic, } C 2 / c \\
& a=16.5626(13) \AA \\
& b=11.4921(10) \AA \\
& c=7.7041(7) \AA \\
& \beta=91.588(8)^{\circ} \\
& V=1465.8(2) \AA^{3} \\
& Z=4
\end{aligned}
$$

\section{Data collection}

Oxford Diffraction Xcalibur E diffractometer

Radiation source: sealed tube $\omega$ scans
$F(000)=750$

$D_{\mathrm{x}}=1.653 \mathrm{Mg} \mathrm{m}^{-3}$

Mo $K \alpha$ radiation, $\lambda=0.71073 \AA$

Cell parameters from 5800 reflections

$\theta=3.4-28.9^{\circ}$

$\mu=1.47 \mathrm{~mm}^{-1}$

$T=150 \mathrm{~K}$

Cut prism, colourless

$0.30 \times 0.20 \times 0.10 \mathrm{~mm}$

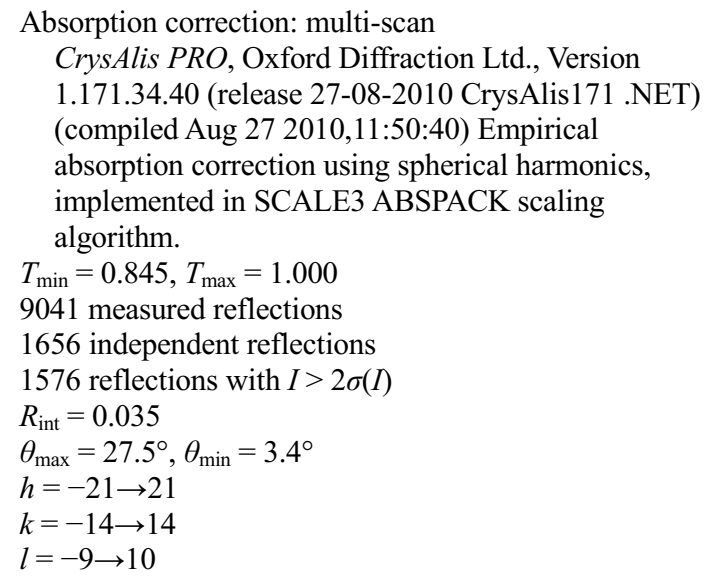

\section{Refinement}

Refinement on $F^{2}$

Least-squares matrix: full

$R\left[F^{2}>2 \sigma\left(F^{2}\right)\right]=0.024$

$w R\left(F^{2}\right)=0.056$

$S=1.11$

1656 reflections

118 parameters

0 restraints
Hydrogen site location: mixed

$\mathrm{H}$ atoms treated by a mixture of independent and constrained refinement

$w=1 /\left[\sigma^{2}\left(F_{\mathrm{o}}^{2}\right)+(0.0227 P)^{2}+0.9652 P\right]$

where $P=\left(F_{\mathrm{o}}{ }^{2}+2 F_{\mathrm{c}}{ }^{2}\right) / 3$

$(\Delta / \sigma)_{\max }=0.001$

$\Delta \rho_{\max }=0.28 \mathrm{e} \AA^{-3}$

$\Delta \rho_{\min }=-0.20$ e $\AA^{-3}$

\section{Special details}

Geometry. All e.s.d.'s (except the e.s.d. in the dihedral angle between two 1.s. planes) are estimated using the full covariance matrix. The cell e.s.d.'s are taken into account individually in the estimation of e.s.d.'s in distances, angles and torsion angles; correlations between e.s.d.'s in cell parameters are only used when they are defined by crystal symmetry. An approximate (isotropic) treatment of cell e.s.d.'s is used for estimating e.s.d.'s involving l.s. planes.

Fractional atomic coordinates and isotropic or equivalent isotropic displacement parameters $\left(\hat{A}^{2}\right)$

\begin{tabular}{llllll}
\hline & $x$ & $y$ & $z$ & $U_{\text {iso }} * / U_{\text {eq }}$ & Occ. $(<1)$ \\
\hline Sr1 & 0.5000 & $0.44698(2)$ & -0.2500 & $0.01216(10)$ & $0.306(3)$ \\
Ca1 & 0.5000 & $0.44698(2)$ & -0.2500 & $0.01216(10)$ & $0.694(3)$ \\
O1 & $0.25637(6)$ & $0.25381(9)$ & $0.26752(13)$ & $0.0210(2)$ &
\end{tabular}




$\begin{array}{lllll}\text { O2 } & 0.37777(6) & 0.39961(8) & 0.26740(13) & 0.0196(2) \\ \text { O3 } & 0.45477(6) & 0.39600(8) & 0.03791(13) & 0.0201(2) \\ \text { O1W } & 0.39752(7) & 0.32033(10) & -0.37681(16) & 0.0260(3) \\ \text { C1 } & 0.30144(8) & 0.18819(12) & 0.15846(17) & 0.0160(3) \\ \text { C2 } & 0.27255(9) & 0.07810(12) & 0.11375(19) & 0.0201(3) \\ \text { H2 } & 0.2220 & 0.0521 & 0.1544 & 0.024^{*} \\ \text { C3 } & 0.31787(10) & 0.00708(13) & 0.00995(19) & 0.0238(3) \\ \text { H3 } & 0.2988 & -0.0687 & -0.0182 & 0.029^{*} \\ \text { C4 } & 0.39121(9) & 0.04474(13) & -0.0543(2) & 0.0237(3) \\ \text { H4 } & 0.4222 & -0.0052 & -0.1246 & 0.028^{*} \\ \text { C5 } & 0.41838(9) & 0.15549(12) & -0.01466(19) & 0.0196(3) \\ \text { H5 } & 0.4678 & 0.1821 & -0.0603 & 0.023^{*} \\ \text { C6 } & 0.37401(8) & 0.22901(12) & 0.09187(17) & 0.0152(3) \\ \text { C7 } & 0.40357(8) & 0.34819(11) & 0.13391(18) & 0.0152(3) \\ \text { H1O } & 0.2827(13) & 0.3191(19) & 0.284(3) & 0.049(6)^{*} \\ \text { H1W } & 0.3521(15) & 0.290(2) & -0.337(3) & 0.062(7)^{*} \\ \text { H2W } & 0.3920(12) & 0.3345(18) & -0.490(3) & 0.046(6)^{*}\end{array}$

Atomic displacement parameters $\left(\AA^{2}\right)$

\begin{tabular}{lllllll}
\hline & $U^{11}$ & $U^{22}$ & $U^{33}$ & $U^{12}$ & $U^{13}$ & $U^{23}$ \\
\hline Sr1 & $0.01084(15)$ & $0.01233(15)$ & $0.01342(16)$ & 0.000 & $0.00272(10)$ & 0.000 \\
Ca1 & $0.01084(15)$ & $0.01233(15)$ & $0.01342(16)$ & 0.000 & $0.00272(10)$ & 0.000 \\
O1 & $0.0183(5)$ & $0.0206(5)$ & $0.0245(6)$ & $-0.0042(4)$ & $0.0069(4)$ & $-0.0028(4)$ \\
O2 & $0.0191(5)$ & $0.0178(5)$ & $0.0222(5)$ & $-0.0023(4)$ & $0.0040(4)$ & $-0.0033(4)$ \\
O3 & $0.0178(5)$ & $0.0169(5)$ & $0.0260(6)$ & $-0.0037(4)$ & $0.0066(4)$ & $0.0000(4)$ \\
O1W & $0.0217(6)$ & $0.0334(6)$ & $0.0232(6)$ & $-0.0055(5)$ & $0.0052(5)$ & $0.0041(5)$ \\
C1 & $0.0163(6)$ & $0.0175(7)$ & $0.0140(7)$ & $-0.0003(5)$ & $0.0002(5)$ & $0.0024(5)$ \\
C2 & $0.0219(7)$ & $0.0199(7)$ & $0.0185(7)$ & $-0.0068(6)$ & $-0.0002(6)$ & $0.0033(6)$ \\
C3 & $0.0355(8)$ & $0.0159(7)$ & $0.0199(8)$ & $-0.0068(6)$ & $-0.0022(6)$ & $-0.0001(6)$ \\
C4 & $0.0301(8)$ & $0.0194(7)$ & $0.0217(8)$ & $0.0018(6)$ & $0.0032(6)$ & $-0.0031(6)$ \\
C5 & $0.0188(7)$ & $0.0196(7)$ & $0.0204(7)$ & $0.0002(5)$ & $0.0028(6)$ & $0.0003(6)$ \\
C6 & $0.0150(6)$ & $0.0149(6)$ & $0.0157(7)$ & $-0.0012(5)$ & $-0.0001(5)$ & $0.0013(5)$ \\
C7 & $0.0114(6)$ & $0.0151(6)$ & $0.0192(7)$ & $0.0004(5)$ & $-0.0006(5)$ & $0.0014(5)$ \\
\end{tabular}

Geometric parameters $\left(\AA,{ }^{o}\right)$

\begin{tabular}{llll}
\hline $\mathrm{Sr} 1-\mathrm{O} 1 W^{\mathrm{i}}$ & $2.4211(11)$ & $\mathrm{O} 3-\mathrm{C} 7$ & $1.2660(16)$ \\
$\mathrm{Sr} 1-\mathrm{O} 1 \mathrm{~W}$ & $2.4211(11)$ & $\mathrm{O} 3-\mathrm{Ca} 1^{\mathrm{ii}}$ & $2.5336(10)$ \\
$\mathrm{Sr} 1-\mathrm{O} 3$ & $2.4324(10)$ & $\mathrm{O} 3-\mathrm{Sr} 1^{\mathrm{ii}}$ & $2.5336(10)$ \\
$\mathrm{Sr} 1-\mathrm{O} 3^{\mathrm{i}}$ & $2.4324(10)$ & $\mathrm{O} 1 \mathrm{~W}-\mathrm{H} 1 \mathrm{~W}$ & $0.89(3)$ \\
$\mathrm{Sr} 1-\mathrm{O} 3^{\text {ii }}$ & $2.5336(10)$ & $\mathrm{O} 1 \mathrm{~W}-\mathrm{H} 2 \mathrm{~W}$ & $0.89(2)$ \\
$\mathrm{Sr} 1-\mathrm{O} 3^{\mathrm{iii}}$ & $2.5336(10)$ & $\mathrm{C} 1-\mathrm{C} 2$ & $1.3925(19)$ \\
$\mathrm{Sr} 1-\mathrm{O} 2^{\mathrm{ii}}$ & $2.6907(10)$ & $\mathrm{C} 1-\mathrm{C} 6$ & $1.4008(19)$ \\
$\mathrm{Sr} 1-\mathrm{O} 2^{\mathrm{iii}}$ & $2.6907(10)$ & $\mathrm{C} 2-\mathrm{C} 3$ & $0.9500(2)$ \\
$\mathrm{Sr} 1-\mathrm{C} 7^{\mathrm{ii}}$ & $2.9686(13)$ & $\mathrm{C} 2-\mathrm{H} 2$ & $1.393(2)$ \\
$\mathrm{Sr} 1-\mathrm{C} 7^{\mathrm{iii}}$ & $2.9686(13)$ & $\mathrm{C} 3-\mathrm{C} 4$ & 0.9500 \\
$\mathrm{Sr} 1-\mathrm{Sr} 1^{\mathrm{ii}}$ & $4.0402(4)$ & $\mathrm{C} 3-\mathrm{H} 3$ & $1.381(2)$ \\
$\mathrm{Sr} 1-\mathrm{Ca} 1^{\mathrm{ii}}$ & $4.0402(4)$ & $\mathrm{C} 4-\mathrm{C} 5$ & 0.9500 \\
$\mathrm{Sr} 1-\mathrm{H} 2 \mathrm{~W}$ & $2.85(2)$ & $\mathrm{C} 4-\mathrm{H} 4$ & $1.4002(19)$ \\
$\mathrm{O} 1-\mathrm{C} 1$ & $1.3662(17)$ & $\mathrm{C} 5-\mathrm{C} 6$ & 0.9500 \\
$\mathrm{O} 1-\mathrm{H} 1 \mathrm{C}$ & $0.87(2)$ & $\mathrm{C} 5-\mathrm{H} 5$ &
\end{tabular}




\begin{tabular}{|c|c|}
\hline $\mathrm{O} 2-\mathrm{C} 7$ & $1.2703(17)$ \\
\hline $\mathrm{O} 2-\mathrm{Ca} 1^{\mathrm{ii}}$ & $2.6906(10)$ \\
\hline $\mathrm{O} 2-\mathrm{Sr} 1^{\mathrm{ii}}$ & $2.6906(10)$ \\
\hline 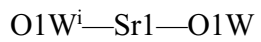 & $106.09(6)$ \\
\hline $\mathrm{O} 1 \mathrm{~W}^{\mathrm{i}}-\mathrm{Sr} 1-\mathrm{O} 3$ & $73.59(4)$ \\
\hline $\mathrm{O} 1 \mathrm{~W}-\mathrm{Sr} 1-\mathrm{O} 3$ & $89.60(4)$ \\
\hline $\mathrm{O} 1 \mathrm{~W}^{\mathrm{i}}-\mathrm{Sr} 1-\mathrm{O}^{\mathrm{i}}$ & $89.60(4)$ \\
\hline $\mathrm{O} 1 \mathrm{~W}-\mathrm{Sr} 1-\mathrm{O}^{\mathrm{i}}$ & $73.59(4)$ \\
\hline $\mathrm{O} 3-\mathrm{Sr} 1-\mathrm{O}^{\mathrm{i}}$ & $152.13(5)$ \\
\hline $\mathrm{O} 1 \mathrm{~W}^{\mathrm{i}}-\mathrm{Sr} 1-\mathrm{O}^{\mathrm{ii}}$ & $88.65(4)$ \\
\hline $\mathrm{O} 1 \mathrm{~W}-\mathrm{Sr} 1-\mathrm{O} 3^{\mathrm{ii}}$ & $151.62(4)$ \\
\hline $\mathrm{O} 3-\mathrm{Sr} 1-\mathrm{O}{ }^{\mathrm{ii}}$ & $71.12(4)$ \\
\hline $\mathrm{O} 3^{\mathrm{i}}-\mathrm{Sr} 1-\mathrm{O} 3^{\mathrm{ii}}$ & $131.81(3)$ \\
\hline 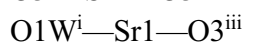 & $151.62(4)$ \\
\hline $\mathrm{O} 1 \mathrm{~W}-\mathrm{Sr} 1-\mathrm{O}^{\mathrm{iii}}$ & $88.65(4)$ \\
\hline $\mathrm{O} 3-\mathrm{Sr} 1-\mathrm{O} 3^{\mathrm{iii}}$ & $131.81(3)$ \\
\hline $\mathrm{O} 3^{\mathrm{i}}-\mathrm{Sr} 1-\mathrm{O} 3^{\mathrm{iii}}$ & $71.12(4)$ \\
\hline 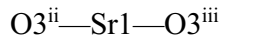 & $89.17(5)$ \\
\hline $\mathrm{O} 1 \mathrm{~W}^{\mathrm{i}}-\mathrm{Sr} 1-\mathrm{O} 2^{\mathrm{ii}}$ & $83.91(3)$ \\
\hline $\mathrm{O} 1 \mathrm{~W}-\mathrm{Sr} 1-\mathrm{O} 2^{\mathrm{ii}}$ & $153.36(4)$ \\
\hline $\mathrm{O} 3-\mathrm{Sr} 1-\mathrm{O} 2^{\mathrm{ii}}$ & $117.03(3)$ \\
\hline $\mathrm{O} 3^{\mathrm{i}}-\mathrm{Sr} 1-\mathrm{O} 2^{\mathrm{ii}}$ & $82.02(3)$ \\
\hline $\mathrm{O} 3^{\mathrm{ii}}-\mathrm{Sr} 1-\mathrm{O} 2^{\mathrm{ii}}$ & $49.91(3)$ \\
\hline $\mathrm{O} 3^{\mathrm{iii}-}-\mathrm{Sr} 1-\mathrm{O} 2^{\mathrm{ii}}$ & $73.18(3)$ \\
\hline $\mathrm{O} 1 \mathrm{~W}^{\mathrm{i}}-\mathrm{Sr} 1-\mathrm{O} 2^{\mathrm{iii}}$ & $153.36(4)$ \\
\hline $\mathrm{O} 1 \mathrm{~W}-\mathrm{Sr} 1-\mathrm{O} 2^{\mathrm{iii}}$ & $83.92(3)$ \\
\hline $\mathrm{O} 3-\mathrm{Sr} 1-\mathrm{O} 2^{\mathrm{iii}}$ & $82.02(3)$ \\
\hline $\mathrm{O} 3^{\mathrm{i}-\mathrm{Sr}} 1-\mathrm{O} 2^{\mathrm{iii}}$ & $117.03(3)$ \\
\hline $\mathrm{O} 3^{\mathrm{ii}}-\mathrm{Sr} 1-\mathrm{O} 2^{\mathrm{iii}}$ & $73.18(3)$ \\
\hline $\mathrm{O} 3^{\mathrm{iii}}-\mathrm{Sr} 1-\mathrm{O} 2^{\mathrm{iii}}$ & $49.91(3)$ \\
\hline $\mathrm{O} 2^{\mathrm{ii}}-\mathrm{Sr} 1-\mathrm{O} 2^{\mathrm{iii}}$ & $98.12(4)$ \\
\hline $\mathrm{O} 1 \mathrm{~W}^{\mathrm{i}}-\mathrm{Sr} 1-\mathrm{C} 7^{\mathrm{ii}}$ & $89.42(4)$ \\
\hline $\mathrm{O} 1 \mathrm{~W}-\mathrm{Sr} 1-\mathrm{C} 7^{\mathrm{ii}}$ & $164.48(4)$ \\
\hline $\mathrm{O} 3-\mathrm{Sr} 1-\mathrm{C} 7^{\mathrm{ii}}$ & $95.33(3)$ \\
\hline $\mathrm{O} 3^{\mathrm{i}}-\mathrm{Sr} 1-\mathrm{C} 7^{\mathrm{ii}}$ & $106.80(4)$ \\
\hline $\mathrm{O}^{3 i}-\mathrm{Sr} 1-\mathrm{C}^{\mathrm{ii}}$ & $25.04(3)$ \\
\hline $\mathrm{O} 3^{\mathrm{iii}}-\mathrm{Sr} 1-\mathrm{C} 7^{\mathrm{ii}^{\mathrm{i}}}$ & $77.09(4)$ \\
\hline $\mathrm{O} 2^{\mathrm{ii}}-\mathrm{Sr} 1-\mathrm{C} 7^{\mathrm{ii}}$ & $25.34(3)$ \\
\hline $\mathrm{O} 2^{\mathrm{iii}}-\mathrm{Sr} 1-\mathrm{C}^{\mathrm{ii}}$ & $82.22(3)$ \\
\hline $\mathrm{O} 1 \mathrm{~W}^{\mathrm{i}}-\mathrm{Sr} 1-\mathrm{C} 7^{\mathrm{iii}}$ & $164.48(4)$ \\
\hline $\mathrm{O} 1 \mathrm{~W}-\mathrm{Sr} 1-\mathrm{C} 7^{\mathrm{iii}}$ & $89.42(4)$ \\
\hline $\mathrm{O} 3-\mathrm{Sr} 1-\mathrm{C} 7^{\mathrm{iii}}$ & $106.80(4)$ \\
\hline $\mathrm{O} 3^{\mathrm{i}}-\mathrm{Sr} 1-\mathrm{C} 7^{\mathrm{iii}}$ & $95.33(4)$ \\
\hline $\mathrm{O}^{\mathrm{ii}}-\mathrm{Sr} 1-\mathrm{C} 7^{\mathrm{iii}}$ & 77.09 (4) \\
\hline $\mathrm{O} 3^{\mathrm{iii}}-\mathrm{Sr} 1-C 7^{\mathrm{iii}}$ & $25.04(3)$ \\
\hline $\mathrm{O} 2^{\mathrm{ii}}-\mathrm{Sr} 1-\mathrm{C}^{\mathrm{iii}}$ & $82.22(3)$ \\
\hline $\mathrm{O} 2^{\mathrm{iii}}-\mathrm{Sr} 1-\mathrm{C} 7^{\mathrm{iii}}$ & $25.34(3)$ \\
\hline $\mathrm{C} 7^{\mathrm{ii}}-\mathrm{Sr} 1-\mathrm{C} 7^{\mathrm{iii}}$ & $75.08(5)$ \\
\hline $\mathrm{O} 1 \mathrm{~W}^{\mathrm{i}}-\mathrm{Sr} 1-\mathrm{Sr} 1^{\mathrm{ii}}$ & $79.35(3)$ \\
\hline $\mathrm{O} 1 \mathrm{~W}-\mathrm{Sr} 1-\mathrm{Sr} 1^{\mathrm{ii}}$ & $123.20(3)$ \\
\hline $\mathrm{O} 3-\mathrm{Sr} 1-\mathrm{Sr} 1^{\mathrm{ii}}$ & $36.40(2)$ \\
\hline
\end{tabular}

$\begin{array}{ll}\mathrm{C} 6-\mathrm{C} 7 & 1.4872(18) \\ \mathrm{C} 7-\mathrm{Ca} 1^{\mathrm{ii}} & 2.9686(13) \\ \mathrm{C} 7-\mathrm{Sr} 1^{1 \mathrm{ii}} & 2.9686(13)\end{array}$

$\mathrm{O}^{\mathrm{iii}}-\mathrm{Sr} 1-\mathrm{Ca}^{\mathrm{ii}} \quad 113.09(3)$

$\mathrm{O} 2^{\mathrm{ii}}-\mathrm{Sr} 1-\mathrm{Ca} 1^{\mathrm{ii}} \quad 82.52(2)$

$\mathrm{O} 2^{\mathrm{iii}}-\mathrm{Sr} 1-\mathrm{Ca}^{\mathrm{ii}} \quad 74.63$ (2)

$\mathrm{C} 7{ }^{\mathrm{ii}}-\mathrm{Sr} 1-\mathrm{Ca} 1^{\mathrm{ii}} \quad 59.19(3)$

$\mathrm{C} 7 \mathrm{7ii}^{\mathrm{ii}}-\mathrm{Sr} 1-\mathrm{Ca} 1^{\mathrm{ii}} \quad 91.94(3)$

$\mathrm{Sr}{ }^{1 i}-\mathrm{Sr} 1-\mathrm{Ca}^{\mathrm{ii}} \quad 0.0$

$\mathrm{O} 1 \mathrm{~W}^{\mathrm{i}-\mathrm{Sr} 1-\mathrm{H} 2 \mathrm{~W}} \quad 114.2$ (4)

$\mathrm{O} 1 \mathrm{~W}-\mathrm{Sr} 1-\mathrm{H} 2 \mathrm{~W}$

$\mathrm{O} 3-\mathrm{Sr} 1-\mathrm{H} 2 \mathrm{~W} \quad 106.2(5)$

$\mathrm{O} 3{ }^{\mathrm{i}}-\mathrm{Sr} 1-\mathrm{H} 2 \mathrm{~W}$

$\mathrm{O} 3{ }^{\mathrm{ii}}-\mathrm{Sr} 1-\mathrm{H} 2 \mathrm{~W}$

$\mathrm{O}^{\mathrm{iii}}-\mathrm{Sr} 1-\mathrm{H} 2 \mathrm{~W}$

$\mathrm{O} 2{ }^{\mathrm{ii}}-\mathrm{Sr} 1-\mathrm{H} 2 \mathrm{~W}$

$\mathrm{O}^{\mathrm{iii}}-\mathrm{Sr} 1-\mathrm{H} 2 \mathrm{~W} \quad 82.5(4)$

$\mathrm{C} 77^{\mathrm{ii}}-\mathrm{Sr} 1-\mathrm{H} 2 \mathrm{~W}$

C7 7 iii-Sr1-H2W $\quad 80.9$ (4)

$\mathrm{Sr} 1{ }^{\mathrm{ii}}-\mathrm{Sr} 1-\mathrm{H} 2 \mathrm{~W} \quad 137.7$ (4)

$\mathrm{Ca} 1{ }^{\mathrm{ii}}-\mathrm{Sr} 1-\mathrm{H} 2 \mathrm{~W} \quad 137.7$ (4)

$\mathrm{Cl}-\mathrm{O} 1-\mathrm{H} 1 \mathrm{O} \quad 106.5(14)$

$\mathrm{C} 7-\mathrm{O} 2-\mathrm{Ca} 1^{\mathrm{ii}}$

$\mathrm{C} 7-\mathrm{O} 2-\mathrm{Sr} 1^{\text {ii }}$

$\mathrm{Ca} 1^{\mathrm{ii}}-\mathrm{O} 2-\mathrm{Sr}^{\mathrm{ii}}$

$\mathrm{C} 7-\mathrm{O} 3-\mathrm{Sr} 1$

C7-O3-Ca $1^{\text {ii }}$

$\mathrm{Sr} 1-\mathrm{O} 3-\mathrm{Ca}{ }^{\mathrm{ii}}$

$\mathrm{C} 7-\mathrm{O} 3-\mathrm{Sr}^{\mathrm{ii}}$

$\mathrm{Sr} 1-\mathrm{O} 3-\mathrm{Sr}^{\mathrm{ii}}$

$\mathrm{Ca} 1^{\mathrm{ii}}-\mathrm{O} 3-\mathrm{Sr}^{\mathrm{ii}}$

$\mathrm{Sr} 1-\mathrm{O} 1 \mathrm{~W}-\mathrm{H} 1 \mathrm{~W}$

$\mathrm{Sr} 1-\mathrm{O} 1 \mathrm{~W}-\mathrm{H} 2 \mathrm{~W}$

$\mathrm{H} 1 \mathrm{~W}-\mathrm{O} 1 \mathrm{~W}-\mathrm{H} 2 \mathrm{~W}$

$\mathrm{O} 1-\mathrm{C} 1-\mathrm{C} 2$

$\mathrm{O} 1-\mathrm{C} 1-\mathrm{C} 6$

$\mathrm{C} 2-\mathrm{C} 1-\mathrm{C} 6$

$\mathrm{C} 3-\mathrm{C} 2-\mathrm{C} 1$

$\mathrm{C} 3-\mathrm{C} 2-\mathrm{H} 2$

$\mathrm{C} 1-\mathrm{C} 2-\mathrm{H} 2$

$\mathrm{C} 2-\mathrm{C} 3-\mathrm{C} 4$

$\mathrm{C} 2-\mathrm{C} 3-\mathrm{H} 3$

$\mathrm{C} 4-\mathrm{C} 3-\mathrm{H} 3$

$\mathrm{C} 5-\mathrm{C} 4-\mathrm{C} 3$

$\mathrm{C} 5-\mathrm{C} 4-\mathrm{H} 4$

$\mathrm{C} 3-\mathrm{C} 4-\mathrm{H} 4$

$\mathrm{C} 4-\mathrm{C} 5-\mathrm{C} 6$

$\mathrm{C} 4-\mathrm{C} 5-\mathrm{H} 5$

$\mathrm{C} 6-\mathrm{C} 5-\mathrm{H} 5$

$\mathrm{C} 5-\mathrm{C} 6-\mathrm{C} 1$

$\mathrm{C} 5-\mathrm{C} 6-\mathrm{C} 7$
$89.66(8)$

$89.66(8)$

0.0

149.93 (9)

97.08 (8)

108.9

$97.08(8)$

108.88 (4)

0.0

$133.3(15)$

109.7 (13)

110.1 (19)

$117.63(12)$

121.97 (12)

120.40 (13)

$119.48(13)$

120.3

120.3

121.07 (14)

119.5

119.5

119.32 (14)

120.3

120.3

120.82 (13)

119.6

119.6

$118.83(13)$

$120.56(12)$ 


\begin{tabular}{|c|c|c|c|}
\hline $\mathrm{O}^{\mathrm{i}}-\mathrm{Sr} 1-\mathrm{Sr} 1^{\mathrm{ii}}$ & $161.85(2)$ & $\mathrm{C} 1-\mathrm{C} 6-\mathrm{C} 7$ & $120.62(12)$ \\
\hline $\mathrm{O} 3^{\mathrm{ii}}-\mathrm{Sr} 1-\mathrm{Sr}^{\mathrm{ii}}$ & $34.73(2)$ & $\mathrm{O} 3-\mathrm{C} 7-\mathrm{O} 2$ & $121.12(12)$ \\
\hline $\mathrm{O} 3{ }^{\mathrm{iii}}-\mathrm{Sr} 1-\mathrm{Sr}^{\mathrm{ii}}$ & $113.09(3)$ & $\mathrm{O} 3-\mathrm{C} 7-\mathrm{C} 6$ & $119.62(12)$ \\
\hline $\mathrm{O} 2^{\mathrm{ii}}-\mathrm{Sr} 1-\mathrm{Sr} 1^{\mathrm{ii}}$ & $82.52(2)$ & $\mathrm{O} 2-\mathrm{C} 7-\mathrm{C} 6$ & $119.25(12)$ \\
\hline $\mathrm{O} 2^{\mathrm{iii}}-\mathrm{Sr} 1-\mathrm{Sr} 1^{\mathrm{ii}}$ & $74.63(2)$ & $\mathrm{O} 3-\mathrm{C} 7-\mathrm{Ca}^{\mathrm{ii}}$ & $57.88(7)$ \\
\hline $\mathrm{C}^{\mathrm{ii}}-\mathrm{Sr} 1-\mathrm{Sr} 1^{\mathrm{ii}}$ & $59.19(3)$ & $\mathrm{O} 2-\mathrm{C} 7-\mathrm{Ca} 1^{\mathrm{ii}}$ & $65.01(7)$ \\
\hline $\mathrm{C} 7^{\mathrm{iii}}-\mathrm{Sr} 1-\mathrm{Sr} 1^{\mathrm{ii}}$ & $91.94(3)$ & $\mathrm{C} 6-\mathrm{C} 7-\mathrm{Ca} 1^{\mathrm{ii}}$ & $165.24(9)$ \\
\hline $\mathrm{O} 1 \mathrm{~W}^{\mathrm{i}}-\mathrm{Sr} 1-\mathrm{Ca}^{\mathrm{ii}}$ & $79.35(3)$ & $\mathrm{O} 3-\mathrm{C} 7-\mathrm{Sr}^{\mathrm{ii}}$ & $57.88(7)$ \\
\hline $\mathrm{O} 1 \mathrm{~W}-\mathrm{Sr} 1-\mathrm{Ca} 1^{\mathrm{ii}}$ & $123.20(3)$ & $\mathrm{O} 2-\mathrm{C} 7-\mathrm{Sr}^{\mathrm{ii}}$ & $65.01(7)$ \\
\hline $\mathrm{O} 3-\mathrm{Sr} 1-\mathrm{Ca} 1^{\mathrm{ii}}$ & $36.40(2)$ & $\mathrm{C} 6-\mathrm{C} 7-\mathrm{Sr}^{\mathrm{ii}}$ & $165.24(9)$ \\
\hline $\mathrm{O} 3^{\mathrm{i}}-\mathrm{Sr} 1-\mathrm{Ca} 1^{\mathrm{ii}}$ & $161.85(2)$ & $\mathrm{Ca} 1^{\mathrm{ii}}-\mathrm{C} 7-\mathrm{Sr}^{\mathrm{ii}}$ & 0.0 \\
\hline $\mathrm{O} 3^{\mathrm{ii}}-\mathrm{Sr} 1-\mathrm{Ca} 1^{\mathrm{ii}}$ & $34.73(2)$ & & \\
\hline $\mathrm{O} 1-\mathrm{C} 1-\mathrm{C} 2-\mathrm{C} 3$ & $-177.25(13)$ & $\mathrm{Sr}^{\mathrm{ii}}-\mathrm{O} 3-\mathrm{C} 7-\mathrm{Ca} 1^{\mathrm{ii}}$ & 0.0 \\
\hline $\mathrm{C} 6-\mathrm{C} 1-\mathrm{C} 2-\mathrm{C} 3$ & $3.1(2)$ & $\mathrm{Sr} 1-\mathrm{O} 3-\mathrm{C} 7-\mathrm{Sr} 1^{\mathrm{ii}}$ & $-149.92(19)$ \\
\hline $\mathrm{C} 1-\mathrm{C} 2-\mathrm{C} 3-\mathrm{C} 4$ & $-1.5(2)$ & $\mathrm{Ca}^{\mathrm{ii}}-\mathrm{O} 3-\mathrm{C} 7-\mathrm{Sr}^{\mathrm{ii}}$ & 0.0 \\
\hline $\mathrm{C} 2-\mathrm{C} 3-\mathrm{C} 4-\mathrm{C} 5$ & $-0.7(2)$ & $\mathrm{Ca} 1^{\mathrm{ii}}-\mathrm{O} 2-\mathrm{C} 7-\mathrm{O} 3$ & $-14.85(13)$ \\
\hline $\mathrm{C} 3-\mathrm{C} 4-\mathrm{C} 5-\mathrm{C} 6$ & $1.4(2)$ & $\mathrm{Sr} 1^{\mathrm{ii}}-\mathrm{O} 2-\mathrm{C} 7-\mathrm{O} 3$ & $-14.85(13)$ \\
\hline $\mathrm{C} 4-\mathrm{C} 5-\mathrm{C} 6-\mathrm{C} 1$ & $0.2(2)$ & $\mathrm{Ca} 1^{\mathrm{ii}}-\mathrm{O} 2-\mathrm{C} 7-\mathrm{C} 6$ & $164.10(10)$ \\
\hline $\mathrm{C} 4-\mathrm{C} 5-\mathrm{C} 6-\mathrm{C} 7$ & $-179.95(13)$ & $\mathrm{Sr} 1^{\mathrm{ii}}-\mathrm{O} 2-\mathrm{C} 7-\mathrm{C} 6$ & $164.10(10)$ \\
\hline $\mathrm{O} 1-\mathrm{C} 1-\mathrm{C} 6-\mathrm{C} 5$ & $177.92(12)$ & $\mathrm{Sr} 1^{\mathrm{ii}}-\mathrm{O} 2-\mathrm{C} 7-\mathrm{Ca} 1^{\mathrm{ii}}$ & 0.0 \\
\hline $\mathrm{C} 2-\mathrm{C} 1-\mathrm{C} 6-\mathrm{C} 5$ & $-2.5(2)$ & $\mathrm{Ca} 1^{\mathrm{ii}}-\mathrm{O} 2-\mathrm{C} 7-\mathrm{Sr}^{\mathrm{ii}}$ & 0.0 \\
\hline $\mathrm{O} 1-\mathrm{C} 1-\mathrm{C} 6-\mathrm{C} 7$ & $-1.9(2)$ & $\mathrm{C} 5-\mathrm{C} 6-\mathrm{C} 7-\mathrm{O} 3$ & $20.16(19)$ \\
\hline $\mathrm{C} 2-\mathrm{C} 1-\mathrm{C} 6-\mathrm{C} 7$ & $177.69(12)$ & $\mathrm{C} 1-\mathrm{C} 6-\mathrm{C} 7-\mathrm{O} 3$ & $-160.01(13)$ \\
\hline $\mathrm{Sr} 1-\mathrm{O} 3-\mathrm{C} 7-\mathrm{O} 2$ & $-134.00(15)$ & $\mathrm{C} 5-\mathrm{C} 6-\mathrm{C} 7-\mathrm{O} 2$ & $-158.80(13)$ \\
\hline $\mathrm{Ca} 1^{\mathrm{ii}}-\mathrm{O} 3-\mathrm{C} 7-\mathrm{O} 2$ & $15.92(13)$ & $\mathrm{C} 1-\mathrm{C} 6-\mathrm{C} 7-\mathrm{O} 2$ & $21.03(19)$ \\
\hline $\mathrm{Sr}^{\mathrm{ii}}-\mathrm{O} 3-\mathrm{C} 7-\mathrm{O} 2$ & $15.92(13)$ & $\mathrm{C} 5-\mathrm{C} 6-\mathrm{C} 7-\mathrm{Ca}^{\mathrm{ii}}$ & $-55.8(4)$ \\
\hline $\mathrm{Sr} 1-\mathrm{O} 3-\mathrm{C} 7-\mathrm{C} 6$ & $47.1(2)$ & $\mathrm{C} 1-\mathrm{C} 6-\mathrm{C} 7-\mathrm{Ca}^{\mathrm{ii}}$ & $124.0(3)$ \\
\hline $\mathrm{Ca}{ }^{\mathrm{ii}}-\mathrm{O} 3-\mathrm{C} 7-\mathrm{C} 6$ & $-163.03(10)$ & $\mathrm{C} 5-\mathrm{C} 6-\mathrm{C} 7-\mathrm{Sr}^{\mathrm{ii}}$ & $-55.8(4)$ \\
\hline $\mathrm{Sr}^{\mathrm{ii}}-\mathrm{O} 3-\mathrm{C} 7-\mathrm{C} 6$ & $-163.03(10)$ & $\mathrm{C} 1-\mathrm{C} 6-\mathrm{C} 7-\mathrm{Sr} 1^{\mathrm{ii}}$ & $124.0(3)$ \\
\hline $\mathrm{Sr} 1-\mathrm{O} 3-\mathrm{C} 7-\mathrm{Ca}^{\mathrm{ii}}$ & $-149.92(19)$ & & \\
\hline
\end{tabular}

Symmetry codes: (i) $-x+1, y,-z-1 / 2$; (ii) $-x+1,-y+1,-z$; (iii) $x,-y+1, z-1 / 2$.

Hydrogen-bond geometry $\left(\AA,{ }^{\circ}\right)$

\begin{tabular}{lllll}
\hline$D-\mathrm{H} \cdots A$ & $D-\mathrm{H}$ & $\mathrm{H} \cdots A$ & $D \cdots A$ & $D-\mathrm{H} \cdots A$ \\
\hline $\mathrm{O} 1-\mathrm{H} 1 O \cdots \mathrm{O} 2$ & $0.87(2)$ & $1.83(2)$ & $2.6174(14)$ & $148(2)$ \\
$\mathrm{O} 1 W-\mathrm{H} 1 W \cdots{ }^{\text {iv }}$ & $0.89(3)$ & $1.95(3)$ & $2.8372(15)$ & $170(2)$ \\
$\mathrm{O} 1 W-\mathrm{H} 2 W \cdots 2^{\mathrm{v}}$ & $0.89(2)$ & $2.02(2)$ & $2.8984(16)$ & $168.7(19)$ \\
\hline
\end{tabular}

Symmetry codes: (iv) $-x+1 / 2,-y+1 / 2,-z$; (v) $x, y, z-1$.

\section{(CaSr5050)}

\section{Crystal data}

$\mathrm{C}_{14} \mathrm{H}_{14} \mathrm{Ca}_{0.47} \mathrm{O}_{8} \mathrm{Sr}_{0.53}$ $M_{r}=375.48$

Monoclinic, $C 2 / c$

$a=16.5994(10) \AA$

$b=11.4832(6) \AA$

$c=7.7650(5) \AA$

$\beta=91.555(5)^{\circ}$
$V=1479.57(15) \AA^{3}$

$Z=4$

$F(000)=766$

$D_{\mathrm{x}}=1.686 \mathrm{Mg} \mathrm{m}^{-3}$

Mo $K \alpha$ radiation, $\lambda=0.71073 \AA$

Cell parameters from 4653 reflections

$\theta=3.4-30.1^{\circ}$ 
$\mu=2.17 \mathrm{~mm}^{-1}$

$T=150 \mathrm{~K}$

\section{Data collection}

Oxford Diffraction Xcalibur E diffractometer

Radiation source: sealed tube $\omega$ scans
Prism, colourless

$0.30 \times 0.20 \times 0.10 \mathrm{~mm}$

\section{Refinement}

Refinement on $F^{2}$

Least-squares matrix: full

$R\left[F^{2}>2 \sigma\left(F^{2}\right)\right]=0.027$

$w R\left(F^{2}\right)=0.058$

$S=1.12$

1691 reflections

118 parameters

0 restraints

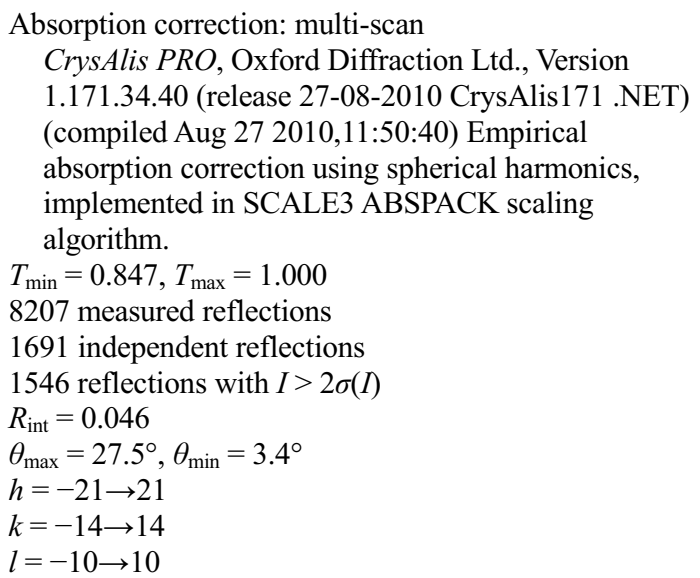

Hydrogen site location: mixed

$\mathrm{H}$ atoms treated by a mixture of independent and constrained refinement

$w=1 /\left[\sigma^{2}\left(F_{\mathrm{o}}^{2}\right)+(0.0194 P)^{2}+0.3827 P\right]$

where $P=\left(F_{\mathrm{o}}^{2}+2 F_{\mathrm{c}}^{2}\right) / 3$

$(\Delta / \sigma)_{\max }<0.001$

$\Delta \rho_{\max }=0.25$ e $\AA^{-3}$

$\Delta \rho_{\min }=-0.28 \mathrm{e} \AA^{-3}$

Special details

Geometry. All e.s.d.'s (except the e.s.d. in the dihedral angle between two 1.s. planes) are estimated using the full covariance matrix. The cell e.s.d.'s are taken into account individually in the estimation of e.s.d.'s in distances, angles and torsion angles; correlations between e.s.d.'s in cell parameters are only used when they are defined by crystal symmetry. An approximate (isotropic) treatment of cell e.s.d.'s is used for estimating e.s.d.'s involving l.s. planes.

Fractional atomic coordinates and isotropic or equivalent isotropic displacement parameters $\left(\AA^{2}\right)$

\begin{tabular}{llllll}
\hline & $x$ & $y$ & $z$ & $U_{\text {iso }} * / U_{\text {eq }}$ & Occ. $(<1)$ \\
\hline Sr1 & 0.5000 & $0.44561(2)$ & -0.2500 & $0.01158(11)$ & $0.529(3)$ \\
Ca1 & 0.5000 & $0.44561(2)$ & -0.2500 & $0.01158(11)$ & $0.471(3)$ \\
O1 & $0.25645(8)$ & $0.25386(12)$ & $0.26716(17)$ & $0.0212(3)$ & $0.0198(3)$ \\
O2 & $0.37738(7)$ & $0.39876(10)$ & $0.26651(17)$ & $0.0204(3)$ & \\
O3 & $0.45461(7)$ & $0.39421(10)$ & $0.03917(17)$ & $0.0277(4)$ & $0.0165(4)$ \\
O1W & $0.39635(9)$ & $0.31743(13)$ & $-0.3803(2)$ & $0.0209(4)$ & $0.025 *$ \\
C1 & $0.30099(11)$ & $0.18734(15)$ & $0.1584(2)$ & $0.0242(5)$ & $0.029 *$ \\
C2 & $0.27182(12)$ & $0.07802(15)$ & $0.1141(2)$ & $0.0240(5)$ \\
H2 & 0.2214 & 0.0522 & 0.1550 & $0.029 *$ \\
C3 & $0.31666(13)$ & $0.00696(16)$ & $0.0102(3)$ & $0.0200(4)$ \\
H3 & 0.2972 & -0.0686 & -0.0185 & $0.024 *$ \\
C4 & $0.38967(12)$ & $0.04380(16)$ & $-0.0531(3)$ & $0.0153(4)$ \\
H4 & 0.4204 & -0.0065 & -0.1229 & \\
C5 & $0.41717(11)$ & $0.15415(15)$ & $-0.0138(2)$ &
\end{tabular}




$\begin{array}{lllll}\text { C7 } & 0.40331(10) & 0.34674(15) & 0.1335(2) & 0.0153(4) \\ \text { H1O } & 0.2830(15) & 0.322(2) & 0.281(3) & 0.053(8)^{*} \\ \text { H1W } & 0.3526(17) & 0.290(2) & -0.338(3) & 0.061(9)^{*} \\ \text { H2W } & 0.3873(16) & 0.334(2) & -0.492(4) & 0.060(9)^{*}\end{array}$

Atomic displacement parameters $\left(\AA^{2}\right)$

\begin{tabular}{lllllll}
\hline & $U^{11}$ & $U^{22}$ & $U^{33}$ & $U^{12}$ & $U^{13}$ & $U^{23}$ \\
\hline Sr1 & $0.01033(17)$ & $0.01192(16)$ & $0.01263(18)$ & 0.000 & $0.00294(11)$ & 0.000 \\
Ca1 & $0.01033(17)$ & $0.01192(16)$ & $0.01263(18)$ & 0.000 & $0.00294(11)$ & 0.000 \\
O1 & $0.0186(7)$ & $0.0224(7)$ & $0.0230(8)$ & $-0.0039(6)$ & $0.0078(6)$ & $-0.0032(6)$ \\
O2 & $0.0193(7)$ & $0.0182(6)$ & $0.0220(8)$ & $-0.0027(5)$ & $0.0045(6)$ & $-0.0042(5)$ \\
O3 & $0.0173(7)$ & $0.0180(6)$ & $0.0264(8)$ & $-0.0043(5)$ & $0.0072(6)$ & $-0.0002(6)$ \\
O1W & $0.0216(8)$ & $0.0379(9)$ & $0.0239(9)$ & $-0.0071(7)$ & $0.0059(7)$ & $0.0032(7)$ \\
C1 & $0.0174(9)$ & $0.0181(9)$ & $0.0140(10)$ & $-0.0001(7)$ & $0.0005(7)$ & $0.0020(7)$ \\
C2 & $0.0225(10)$ & $0.0212(9)$ & $0.0190(11)$ & $-0.0082(8)$ & $-0.0002(8)$ & $0.0036(8)$ \\
C3 & $0.0354(12)$ & $0.0161(9)$ & $0.0209(12)$ & $-0.0072(9)$ & $-0.0020(9)$ & $-0.0003(8)$ \\
C4 & $0.0302(11)$ & $0.0191(9)$ & $0.0228(11)$ & $0.0019(8)$ & $0.0023(9)$ & $-0.0032(8)$ \\
C5 & $0.0187(10)$ & $0.0205(9)$ & $0.0210(11)$ & $-0.0002(8)$ & $0.0033(8)$ & $-0.0001(8)$ \\
C6 & $0.0143(9)$ & $0.0160(9)$ & $0.0155(10)$ & $-0.0001(7)$ & $0.0004(7)$ & $0.0020(7)$ \\
C7 & $0.0108(9)$ & $0.0175(9)$ & $0.0176(10)$ & $0.0015(7)$ & $-0.0005(7)$ & $0.0018(7)$ \\
\end{tabular}

Geometric parameters $\left(\AA,{ }^{\circ}\right)$

\begin{tabular}{|c|c|c|c|}
\hline $\mathrm{Sr} 1-\mathrm{O} 3^{\mathrm{i}}$ & $2.4596(13)$ & $\mathrm{O} 3-\mathrm{C} 7$ & $1.262(2)$ \\
\hline $\mathrm{Sr} 1-\mathrm{O} 3$ & $2.4597(13)$ & $\mathrm{O} 3-\mathrm{Ca} 1^{\mathrm{ii}}$ & $2.5621(13)$ \\
\hline $\mathrm{Sr} 1-\mathrm{O} 1 \mathrm{~W}^{\mathrm{i}}$ & $2.4608(15)$ & $\mathrm{O} 3-\mathrm{Sr}^{\mathrm{ii}}$ & $2.5621(13)$ \\
\hline $\mathrm{Sr} 1-\mathrm{O} 1 \mathrm{~W}$ & $2.4608(15)$ & $\mathrm{O} 1 \mathrm{~W}-\mathrm{H} 1 \mathrm{~W}$ & $0.86(3)$ \\
\hline $\mathrm{Sr} 1-\mathrm{O} 3^{\mathrm{ii}}$ & $2.5621(13)$ & $\mathrm{O} 1 \mathrm{~W}-\mathrm{H} 2 \mathrm{~W}$ & $0.90(3)$ \\
\hline $\mathrm{Sr} 1-\mathrm{O} 3^{\mathrm{iii}}$ & $2.5621(13)$ & $\mathrm{C} 1-\mathrm{C} 2$ & $1.385(2)$ \\
\hline $\mathrm{Sr} 1-\mathrm{O} 2^{\mathrm{ii}}$ & $2.7143(12)$ & $\mathrm{C} 1-\mathrm{C} 6$ & $1.398(2)$ \\
\hline $\mathrm{Sr} 1-\mathrm{O} 2^{\mathrm{iii}}$ & $2.7143(12)$ & $\mathrm{C} 2-\mathrm{C} 3$ & $1.380(3)$ \\
\hline $\mathrm{Sr} 1-\mathrm{C} 7^{\mathrm{ii}}$ & $3.0001(18)$ & $\mathrm{C} 2-\mathrm{H} 2$ & 0.9500 \\
\hline $\mathrm{Sr} 1-\mathrm{C}^{\mathrm{iii}}$ & $3.0001(18)$ & $\mathrm{C} 3-\mathrm{C} 4$ & $1.386(3)$ \\
\hline $\mathrm{Sr} 1-\mathrm{Sr} 1^{\mathrm{ii}}$ & $4.0785(3)$ & $\mathrm{C} 3-\mathrm{H} 3$ & 0.9500 \\
\hline $\mathrm{Sr} 1-\mathrm{Ca}^{\mathrm{ii}}$ & $4.0785(3)$ & $\mathrm{C} 4-\mathrm{C} 5$ & $1.378(2)$ \\
\hline $\mathrm{Sr} 1-\mathrm{H} 2 \mathrm{~W}$ & $2.92(3)$ & $\mathrm{C} 4-\mathrm{H} 4$ & 0.9500 \\
\hline $\mathrm{O} 1-\mathrm{C} 1$ & $1.370(2)$ & $\mathrm{C} 5-\mathrm{C} 6$ & $1.401(2)$ \\
\hline $\mathrm{O} 1-\mathrm{H} 1 \mathrm{O}$ & $0.90(3)$ & $\mathrm{C} 5-\mathrm{H} 5$ & 0.9500 \\
\hline $\mathrm{O} 2-\mathrm{C} 7$ & $1.278(2)$ & $\mathrm{C} 6-\mathrm{C} 7$ & $1.483(2)$ \\
\hline $\mathrm{O} 2-\mathrm{Ca}^{\mathrm{ii}}$ & $2.7143(12)$ & $\mathrm{C} 7-\mathrm{Ca}^{\mathrm{ii}}$ & $3.0001(18)$ \\
\hline $\mathrm{O} 2-\mathrm{Sr} 1^{\mathrm{ii}}$ & $2.7143(12)$ & $\mathrm{C} 7-\mathrm{Sr} 1^{\mathrm{ii}}$ & $3.0001(18)$ \\
\hline $\mathrm{O} 3{ }^{\mathrm{i}}-\mathrm{Sr} 1-\mathrm{O} 3$ & $152.23(6)$ & $\mathrm{O} 33^{\mathrm{iii}}-\mathrm{Sr} 1-\mathrm{Ca}^{\mathrm{ii}}$ & $112.38(3)$ \\
\hline $\mathrm{O} 3^{\mathrm{i}}-\mathrm{Sr} 1-\mathrm{O} 1 \mathrm{~W}^{\mathrm{i}}$ & $90.21(5)$ & $\mathrm{O} 2^{\mathrm{ii}}-\mathrm{Sr} 1-\mathrm{Ca} 1^{\mathrm{ii}}$ & $82.11(3)$ \\
\hline $\mathrm{O} 3-\mathrm{Sr} 1-\mathrm{O} 1 \mathrm{~W}^{\mathrm{i}}$ & $73.10(5)$ & $\mathrm{O} 2^{\mathrm{iii}}-\mathrm{Sr} 1-\mathrm{Ca} 1^{\mathrm{ii}}$ & $74.57(3)$ \\
\hline $\mathrm{O} 3^{\mathrm{i}}-\mathrm{Sr} 1-\mathrm{O} 1 \mathrm{~W}$ & $73.10(5)$ & $\mathrm{C} 7^{\mathrm{ii}}-\mathrm{Sr} 1-\mathrm{Ca} 1^{\mathrm{ii}}$ & $58.90(4)$ \\
\hline $\mathrm{O} 3-\mathrm{Sr} 1-\mathrm{O} 1 \mathrm{~W}$ & $90.21(5)$ & $\mathrm{C} 7^{\mathrm{iii}}-\mathrm{Sr} 1-\mathrm{Ca} 1^{\mathrm{ii}}$ & $91.70(4)$ \\
\hline $\mathrm{O} 1 \mathrm{~W}^{\mathrm{i}}-\mathrm{Sr} 1-\mathrm{O} 1 \mathrm{~W}$ & $106.53(7)$ & $\mathrm{Sr} 1^{\mathrm{ii}}-\mathrm{Sr} 1-\mathrm{Ca} 1^{\mathrm{ii}}$ & $0.000(8)$ \\
\hline $\mathrm{O}^{\mathrm{i}}-\mathrm{Sr} 1-\mathrm{O}^{\mathrm{ii}}$ & $131.57(4)$ & $\mathrm{O} 3^{\mathrm{i}}-\mathrm{Sr} 1-\mathrm{H} 2 \mathrm{~W}$ & $60.8(5)$ \\
\hline $\mathrm{O} 3-\mathrm{Sr} 1-\mathrm{O}^{\mathrm{ii}}$ & $71.40(4)$ & $\mathrm{O} 3-\mathrm{Sr} 1-\mathrm{H} 2 \mathrm{~W}$ & $106.1(5)$ \\
\hline $\mathrm{O} 1 \mathrm{~W}^{\mathrm{i}}-\mathrm{Sr} 1-\mathrm{O} 3^{\mathrm{ii}}$ & $88.66(5)$ & $\mathrm{O} 1 \mathrm{~W}^{\mathrm{i}}-\mathrm{Sr} 1-\mathrm{H} 2 \mathrm{~W}$ & $115.5(5)$ \\
\hline
\end{tabular}




\begin{tabular}{|c|c|}
\hline $\mathrm{O} 1 \mathrm{~W}-\mathrm{Sr} 1-\mathrm{O} 3^{\mathrm{ii}}$ & $151.92(5)$ \\
\hline $\mathrm{O} 3^{\mathrm{i}}-\mathrm{Sr} 1-\mathrm{O} 3^{\mathrm{iii}}$ & $71.40(4)$ \\
\hline $\mathrm{O} 3-\mathrm{Sr} 1-\mathrm{O} 3^{\mathrm{iii}}$ & $131.57(4)$ \\
\hline $\mathrm{O} 1 \mathrm{~W}^{\mathrm{i}}-\mathrm{Sr} 1-\mathrm{O} 3^{\mathrm{iii}}$ & $151.92(5)$ \\
\hline $\mathrm{O} 1 \mathrm{~W}-\mathrm{Sr} 1-\mathrm{O} 3^{\mathrm{iii}}$ & $88.66(5)$ \\
\hline $\mathrm{O} 3^{\mathrm{ii}}-\mathrm{Sr} 1-\mathrm{O} 3^{\mathrm{iii}}$ & $88.23(6)$ \\
\hline $\mathrm{O} 3^{\mathrm{i}}-\mathrm{Sr} 1-\mathrm{O} 2^{\mathrm{ii}}$ & $82.31(4)$ \\
\hline $\mathrm{O} 3-\mathrm{Sr} 1-\mathrm{O} 2^{\mathrm{ii}}$ & $116.73(4)$ \\
\hline $\mathrm{O} 1 \mathrm{~W}^{\mathrm{i}}-\mathrm{Sr} 1-\mathrm{O} 2^{\mathrm{ii}}$ & $84.05(4)$ \\
\hline $\mathrm{O} 1 \mathrm{~W}-\mathrm{Sr} 1-\mathrm{O} 2^{\mathrm{ii}}$ & $153.03(5)$ \\
\hline $\mathrm{O} 3^{\mathrm{ii}}-\mathrm{Sr} 1-\mathrm{O} 2^{\mathrm{ii}}$ & $49.43(4)$ \\
\hline $\mathrm{O} 3^{\mathrm{iii}}-\mathrm{Sr} 1-\mathrm{O} 2^{\mathrm{ii}}$ & $72.84(4)$ \\
\hline $\mathrm{O} 3^{\mathrm{i}}-\mathrm{Sr} 1-\mathrm{O} 2^{\mathrm{iii}}$ & $116.73(4)$ \\
\hline $\mathrm{O} 3-\mathrm{Sr} 1-\mathrm{O} 2^{\mathrm{iii}}$ & $82.31(4)$ \\
\hline $\mathrm{O} 1 \mathrm{~W}^{\mathrm{i}}-\mathrm{Sr} 1-\mathrm{O} 2^{\mathrm{iii}}$ & $153.03(5)$ \\
\hline $\mathrm{O} 1 \mathrm{~W}-\mathrm{Sr} 1-\mathrm{O} 2^{\mathrm{iii}}$ & $84.05(4)$ \\
\hline $\mathrm{O} 3^{\mathrm{ii}}-\mathrm{Sr} 1-\mathrm{O} 2^{\mathrm{iii}}$ & $72.84(4)$ \\
\hline $\mathrm{O} 3^{\mathrm{iii}}-\mathrm{Sr} 1-\mathrm{O} 2^{\mathrm{iii}}$ & $49.43(4)$ \\
\hline $\mathrm{O} 2^{\mathrm{ii}}-\mathrm{Sr} 1-\mathrm{O} 2^{\mathrm{iii}}$ & $97.64(5)$ \\
\hline $\mathrm{O} 3^{\mathrm{i}}-\mathrm{Sr} 1-\mathrm{C}^{\mathrm{ii}}$ & $106.94(4)$ \\
\hline $\mathrm{O} 3-\mathrm{Sr} 1-\mathrm{C}^{\mathrm{ii}}$ & $95.17(5)$ \\
\hline $\mathrm{O} 1 \mathrm{~W}^{\mathrm{i}}-\mathrm{Sr} 1-\mathrm{C}^{\mathrm{ii}}$ & $89.38(5)$ \\
\hline $\mathrm{O} 1 \mathrm{~W}-\mathrm{Sr} 1-\mathrm{C}^{\mathrm{ii}}$ & $164.08(5)$ \\
\hline $\mathrm{O} 3^{\mathrm{ii}}-\mathrm{Sr} 1-\mathrm{C} 7^{\mathrm{ii}}$ & $24.65(4)$ \\
\hline $\mathrm{O} 3^{\mathrm{iii}}-\mathrm{Sr} 1-\mathrm{C}^{\mathrm{ii}}$ & $76.56(4)$ \\
\hline 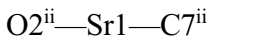 & $25.21(4)$ \\
\hline $\mathrm{O} 2^{\mathrm{iii}}-\mathrm{Sr} 1-\mathrm{C}^{\mathrm{ii}}$ & $81.84(4)$ \\
\hline $\mathrm{O} 3^{\mathrm{i}}-\mathrm{Sr} 1-\mathrm{C} 7^{\mathrm{iii}}$ & $95.17(5)$ \\
\hline $\mathrm{O} 3-\mathrm{Sr} 1-\mathrm{C} 7^{\mathrm{iii}}$ & $106.94(4)$ \\
\hline $\mathrm{O} 1 \mathrm{~W}^{\mathrm{i}}-\mathrm{Sr} 1-\mathrm{C}^{\mathrm{iii}}$ & $164.08(5)$ \\
\hline $\mathrm{O} 1 \mathrm{~W}-\mathrm{Sr} 1-\mathrm{C}^{\mathrm{iii}}$ & $89.38(5)$ \\
\hline $\mathrm{O} 3^{\mathrm{ii}}-\mathrm{Sr} 1-\mathrm{C} 7^{\mathrm{iii}}$ & $76.56(4)$ \\
\hline $\mathrm{O} 3^{\mathrm{iii}}-\mathrm{Sr} 1-\mathrm{C}^{\mathrm{iii}}$ & $24.65(4)$ \\
\hline $\mathrm{O} 2^{\mathrm{ii}}-\mathrm{Sr} 1-\mathrm{C}^{\mathrm{iii}}$ & $81.84(4)$ \\
\hline 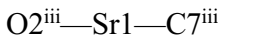 & $25.21(4)$ \\
\hline $\mathrm{C}^{\mathrm{ii}}-\mathrm{Sr} 1-\mathrm{C}^{\mathrm{iii}}$ & $74.73(7)$ \\
\hline $\mathrm{O} 3^{\mathrm{i}}-\mathrm{Sr} 1-\mathrm{Sr} 1^{\mathrm{ii}}$ & $161.89(3)$ \\
\hline $\mathrm{O} 3-\mathrm{Sr} 1-\mathrm{Sr} 1^{\mathrm{ii}}$ & $36.54(3)$ \\
\hline $\mathrm{O} 1 \mathrm{~W}^{\mathrm{i}}-\mathrm{Sr} 1-\mathrm{Sr}^{\mathrm{ii}}$ & $79.04(4)$ \\
\hline $\mathrm{O} 1 \mathrm{~W}-\mathrm{Sr} 1-\mathrm{Sr} 1^{\mathrm{ii}}$ & $123.81(4)$ \\
\hline $\mathrm{O} 3^{\mathrm{ii}}-\mathrm{Sr} 1-\mathrm{Sr} 1^{\mathrm{ii}}$ & $34.86(3)$ \\
\hline $\mathrm{O} 3^{\mathrm{iii}}-\mathrm{Sr} 1-\mathrm{Sr} 1^{\mathrm{ii}}$ & $112.38(3)$ \\
\hline $\mathrm{O} 2^{\mathrm{ii}}-\mathrm{Sr} 1-\mathrm{Sr} 1^{\mathrm{ii}}$ & $82.11(3)$ \\
\hline $\mathrm{O} 2^{\mathrm{iii}}-\mathrm{Sr} 1-\mathrm{Sr} 1^{\mathrm{ii}}$ & $74.57(3)$ \\
\hline $\mathrm{C} 7^{\mathrm{ii}}-\mathrm{Sr} 1-\mathrm{Sr} 1^{\mathrm{ii}}$ & $58.90(4)$ \\
\hline $\mathrm{C} 7^{\mathrm{iii}}-\mathrm{Sr} 1-\mathrm{Sr} 1^{\mathrm{ii}}$ & $91.70(4)$ \\
\hline $\mathrm{O} 3^{\mathrm{i}}-\mathrm{Sr} 1-\mathrm{Ca} 1^{\mathrm{ii}}$ & $161.89(3)$ \\
\hline $\mathrm{O} 3-\mathrm{Sr} 1-\mathrm{Ca} 1^{\mathrm{ii}}$ & $36.54(3)$ \\
\hline $\mathrm{O} 1 \mathrm{~W}^{\mathrm{i}}-\mathrm{Sr} 1-\mathrm{Ca} 1^{\mathrm{ii}}$ & $79.04(4)$ \\
\hline $\mathrm{O} 1 \mathrm{~W}-\mathrm{Sr} 1-\mathrm{Ca} 1^{\mathrm{ii}}$ & $123.81(4)$ \\
\hline $\mathrm{O} 3^{\mathrm{ii}}-\mathrm{Sr} 1-\mathrm{Ca} 1^{\mathrm{ii}}$ & $34.86(3)$ \\
\hline
\end{tabular}

\begin{tabular}{|c|c|}
\hline $\mathrm{O} 1 \mathrm{~W}-\mathrm{Sr} 1-\mathrm{H} 2 \mathrm{~W}$ & $16.7(5)$ \\
\hline $\mathrm{O} 33^{\mathrm{ii}}-\mathrm{Sr} 1-\mathrm{H} 2 \mathrm{~W}$ & $154.3(5)$ \\
\hline $\mathrm{O} 3^{\mathrm{iii}}-\mathrm{Sr} 1-\mathrm{H} 2 \mathrm{~W}$ & $74.4(5)$ \\
\hline $\mathrm{O} 2^{\mathrm{ii}}-\mathrm{Sr} 1-\mathrm{H} 2 \mathrm{~W}$ & $136.7(5)$ \\
\hline $\mathrm{O} 2{ }^{\mathrm{iii}}-\mathrm{Sr} 1-\mathrm{H} 2 \mathrm{~W}$ & $81.5(5)$ \\
\hline $\mathrm{C}^{\mathrm{ii}}-\mathrm{Sr} 1-\mathrm{H} 2 \mathrm{~W}$ & $150.8(5)$ \\
\hline $\mathrm{C} 7^{\mathrm{iii}}-\mathrm{Sr} 1-\mathrm{H} 2 \mathrm{~W}$ & $80.0(5)$ \\
\hline $\mathrm{Sr} 1 \stackrel{\mathrm{ii}}{-} \mathrm{Sr} 1-\mathrm{H} 2 \mathrm{~W}$ & $137.1(5)$ \\
\hline $\mathrm{Ca} 1^{\mathrm{ii}}-\mathrm{Sr} 1-\mathrm{H} 2 \mathrm{~W}$ & $137.1(5)$ \\
\hline $\mathrm{C} 1-\mathrm{O} 1-\mathrm{H} 1 \mathrm{O}$ & $106.5(15)$ \\
\hline $\mathrm{C} 7-\mathrm{O} 2-\mathrm{Ca} 1^{\mathrm{ii}}$ & $90.00(10)$ \\
\hline $\mathrm{C} 7-\mathrm{O} 2-\mathrm{Sr} 1^{\mathrm{ii}}$ & $90.00(10)$ \\
\hline $\mathrm{Ca} 1^{\mathrm{ii}}-\mathrm{O} 2-\mathrm{Sr}^{\mathrm{ii}}$ & 0.0 \\
\hline $\mathrm{C} 7-\mathrm{O} 3-\mathrm{Sr} 1$ & $149.53(12)$ \\
\hline $\mathrm{C} 7-\mathrm{O} 3-\mathrm{Ca} 1^{\mathrm{ii}}$ & $97.49(11)$ \\
\hline $\mathrm{Sr} 1-\mathrm{O} 3-\mathrm{Ca} 1^{\mathrm{ii}}$ & 108.6 \\
\hline $\mathrm{C} 7-\mathrm{O} 3-\mathrm{Sr} 1^{\mathrm{ii}}$ & $97.49(11)$ \\
\hline $\mathrm{Sr} 1-\mathrm{O} 3-\mathrm{Sr} 1^{\mathrm{ii}}$ & $108.60(4)$ \\
\hline $\mathrm{Ca} 1^{\mathrm{ii}}-\mathrm{O} 3-\mathrm{Sr} 1^{\mathrm{ii}}$ & 0.0 \\
\hline $\mathrm{Sr} 1-\mathrm{O} 1 \mathrm{~W}-\mathrm{H} 1 \mathrm{~W}$ & $130.5(18)$ \\
\hline $\mathrm{Sr} 1-\mathrm{O} 1 \mathrm{~W}-\mathrm{H} 2 \mathrm{~W}$ & $111.7(17)$ \\
\hline $\mathrm{H} 1 \mathrm{~W}-\mathrm{O} 1 \mathrm{~W}-\mathrm{H} 2 \mathrm{~W}$ & $109(2)$ \\
\hline $\mathrm{O} 1-\mathrm{C} 1-\mathrm{C} 2$ & $117.85(16)$ \\
\hline $\mathrm{O} 1-\mathrm{C} 1-\mathrm{C} 6$ & $121.47(16)$ \\
\hline $\mathrm{C} 2-\mathrm{C} 1-\mathrm{C} 6$ & $120.68(17)$ \\
\hline $\mathrm{C} 3-\mathrm{C} 2-\mathrm{C} 1$ & $119.37(18)$ \\
\hline $\mathrm{C} 3-\mathrm{C} 2-\mathrm{H} 2$ & 120.3 \\
\hline $\mathrm{C} 1-\mathrm{C} 2-\mathrm{H} 2$ & 120.3 \\
\hline $\mathrm{C} 2-\mathrm{C} 3-\mathrm{C} 4$ & $121.17(18)$ \\
\hline $\mathrm{C} 2-\mathrm{C} 3-\mathrm{H} 3$ & 119.4 \\
\hline $\mathrm{C} 4-\mathrm{C} 3-\mathrm{H} 3$ & 119.4 \\
\hline $\mathrm{C} 5-\mathrm{C} 4-\mathrm{C} 3$ & $119.30(18)$ \\
\hline $\mathrm{C} 5-\mathrm{C} 4-\mathrm{H} 4$ & 120.4 \\
\hline $\mathrm{C} 3-\mathrm{C} 4-\mathrm{H} 4$ & 120.4 \\
\hline $\mathrm{C} 4-\mathrm{C} 5-\mathrm{C} 6$ & $120.88(17)$ \\
\hline $\mathrm{C} 4-\mathrm{C} 5-\mathrm{H} 5$ & 119.6 \\
\hline $\mathrm{C} 6-\mathrm{C} 5-\mathrm{H} 5$ & 119.6 \\
\hline $\mathrm{C} 1-\mathrm{C} 6-\mathrm{C} 5$ & $118.54(16)$ \\
\hline $\mathrm{C} 1-\mathrm{C} 6-\mathrm{C} 7$ & $121.03(16)$ \\
\hline $\mathrm{C} 5-\mathrm{C} 6-\mathrm{C} 7$ & $120.43(16)$ \\
\hline $\mathrm{O} 3-\mathrm{C} 7-\mathrm{O} 2$ & $120.98(16)$ \\
\hline $\mathrm{O} 3-\mathrm{C} 7-\mathrm{C} 6$ & $120.06(16)$ \\
\hline $\mathrm{O} 2-\mathrm{C} 7-\mathrm{C} 6$ & $118.95(16)$ \\
\hline $\mathrm{O} 3-\mathrm{C} 7-\mathrm{Ca} 1^{\mathrm{ii}}$ & $57.86(9)$ \\
\hline $\mathrm{O} 2-\mathrm{C} 7-\mathrm{Ca} 1^{\mathrm{ii}}$ & $64.79(9)$ \\
\hline $\mathrm{C} 6-\mathrm{C} 7-\mathrm{Ca} 1^{\mathrm{ii}}$ & $165.59(12)$ \\
\hline $\mathrm{O} 3-\mathrm{C} 7-\mathrm{Sr} 1^{\mathrm{ii}}$ & $57.86(9)$ \\
\hline $\mathrm{O} 2-\mathrm{C} 7-\mathrm{Sr}^{\mathrm{ii}}$ & $64.79(9)$ \\
\hline $\mathrm{C} 6-\mathrm{C} 7-\mathrm{Sr} 1^{\mathrm{ii}}$ & $165.59(12)$ \\
\hline $\mathrm{Ca} 1^{\mathrm{ii}}-\mathrm{C} 7-\mathrm{Sr} 1^{\mathrm{ii}}$ & 0.0 \\
\hline
\end{tabular}




\begin{tabular}{|c|c|c|c|}
\hline $\mathrm{O} 1-\mathrm{C} 1-\mathrm{C} 2-\mathrm{C} 3$ & $-177.53(17)$ & $\mathrm{Sr} 1^{\mathrm{ii}}-\mathrm{O} 3-\mathrm{C} 7-\mathrm{Ca}{ }^{\mathrm{ii}}$ & 0.0 \\
\hline $\mathrm{C} 6-\mathrm{C} 1-\mathrm{C} 2-\mathrm{C} 3$ & $2.7(3)$ & $\mathrm{Sr} 1-\mathrm{O} 3-\mathrm{C} 7-\mathrm{Sr} 1^{\mathrm{ii}}$ & $-149.1(2)$ \\
\hline $\mathrm{C} 1-\mathrm{C} 2-\mathrm{C} 3-\mathrm{C} 4$ & $-1.1(3)$ & $\mathrm{Ca} 1^{\mathrm{ii}}-\mathrm{O} 3-\mathrm{C} 7-\mathrm{Sr}^{1 i}$ & 0.0 \\
\hline $\mathrm{C} 2-\mathrm{C} 3-\mathrm{C} 4-\mathrm{C} 5$ & $-1.1(3)$ & $\mathrm{Ca} 1^{\mathrm{ii}}-\mathrm{O} 2-\mathrm{C} 7-\mathrm{O} 3$ & $-14.40(17)$ \\
\hline $\mathrm{C} 3-\mathrm{C} 4-\mathrm{C} 5-\mathrm{C} 6$ & $1.8(3)$ & $\mathrm{Sr} 1^{\mathrm{ii}}-\mathrm{O} 2-\mathrm{C} 7-\mathrm{O} 3$ & $-14.40(17)$ \\
\hline $\mathrm{O} 1-\mathrm{C} 1-\mathrm{C} 6-\mathrm{C} 5$ & $178.20(16)$ & $\mathrm{Ca} 1^{\mathrm{ii}}-\mathrm{O} 2-\mathrm{C} 7-\mathrm{C} 6$ & $164.36(14)$ \\
\hline $\mathrm{C} 2-\mathrm{C} 1-\mathrm{C} 6-\mathrm{C} 5$ & $-2.1(3)$ & $\mathrm{Sr} 1^{\mathrm{ii}}-\mathrm{O} 2-\mathrm{C} 7-\mathrm{C} 6$ & $164.36(14)$ \\
\hline $\mathrm{O} 1-\mathrm{C} 1-\mathrm{C} 6-\mathrm{C} 7$ & $-2.4(3)$ & $\mathrm{Sr} 1^{\mathrm{ii}}-\mathrm{O} 2-\mathrm{C} 7-\mathrm{Ca} 1^{\mathrm{ii}}$ & 0.0 \\
\hline $\mathrm{C} 2-\mathrm{C} 1-\mathrm{C} 6-\mathrm{C} 7$ & $177.32(17)$ & $\mathrm{Ca}{ }^{\mathrm{ii}}-\mathrm{O} 2-\mathrm{C} 7-\mathrm{Sr}{ }^{\mathrm{ii}}$ & 0.0 \\
\hline $\mathrm{C} 4-\mathrm{C} 5-\mathrm{C} 6-\mathrm{C} 1$ & $-0.2(3)$ & $\mathrm{C} 1-\mathrm{C} 6-\mathrm{C} 7-\mathrm{O} 3$ & $-160.07(17)$ \\
\hline $\mathrm{C} 4-\mathrm{C} 5-\mathrm{C} 6-\mathrm{C} 7$ & $-179.59(18)$ & $\mathrm{C} 5-\mathrm{C} 6-\mathrm{C} 7-\mathrm{O} 3$ & $19.3(3)$ \\
\hline $\mathrm{Sr} 1-\mathrm{O} 3-\mathrm{C} 7-\mathrm{O} 2$ & $-133.67(19)$ & $\mathrm{C} 1-\mathrm{C} 6-\mathrm{C} 7-\mathrm{O} 2$ & $21.2(3)$ \\
\hline $\mathrm{Ca} 1^{\mathrm{ii}}-\mathrm{O} 3-\mathrm{C} 7-\mathrm{O} 2$ & $15.42(18)$ & $\mathrm{C} 5-\mathrm{C} 6-\mathrm{C} 7-\mathrm{O} 2$ & $-159.47(17)$ \\
\hline $\mathrm{Sr}^{\mathrm{ii}}-\mathrm{O} 3-\mathrm{C} 7-\mathrm{O} 2$ & $15.42(18)$ & $\mathrm{C} 1-\mathrm{C} 6-\mathrm{C} 7-\mathrm{Ca}^{\mathrm{ii}}$ & $122.5(5)$ \\
\hline $\mathrm{Sr} 1-\mathrm{O} 3-\mathrm{C} 7-\mathrm{C} 6$ & $47.6(3)$ & $\mathrm{C} 5-\mathrm{C} 6-\mathrm{C} 7-\mathrm{Ca}^{\mathrm{ii}}$ & $-58.1(6)$ \\
\hline $\mathrm{Ca}{ }^{\mathrm{ii}}-\mathrm{O} 3-\mathrm{C} 7-\mathrm{C} 6$ & $-163.33(14)$ & $\mathrm{C} 1-\mathrm{C} 6-\mathrm{C} 7-\mathrm{Sr}^{\mathrm{ii}}$ & $122.5(5)$ \\
\hline $\mathrm{Sr}^{\mathrm{ii}}-\mathrm{O} 3-\mathrm{C} 7-\mathrm{C} 6$ & $-163.33(14)$ & $\mathrm{C} 5-\mathrm{C} 6-\mathrm{C} 7-\mathrm{Sr} 1^{\mathrm{ii}}$ & $-58.1(6)$ \\
\hline $\mathrm{Sr} 1-\mathrm{O} 3-\mathrm{C} 7-\mathrm{Ca}^{\mathrm{ii}}$ & $-149.1(2)$ & & \\
\hline
\end{tabular}

Symmetry codes: (i) $-x+1, y,-z-1 / 2$; (ii) $-x+1,-y+1,-z$; (iii) $x,-y+1, z-1 / 2$.

Hydrogen-bond geometry $\left(\AA,{ }^{\circ}\right)$

\begin{tabular}{lllll}
\hline$D-\mathrm{H} \cdots A$ & $D-\mathrm{H}$ & $\mathrm{H} \cdots A$ & $D \cdots A$ & $D-\mathrm{H} \cdots A$ \\
\hline $\mathrm{O} 1-\mathrm{H} 1 O \cdots \mathrm{O} 2$ & $0.90(3)$ & $1.81(3)$ & $2.6075(17)$ & $147(2)$ \\
$\mathrm{O} 1 W-\mathrm{H} 1 W \cdots{ }^{\mathrm{iv}}$ & $0.86(3)$ & $1.97(3)$ & $2.8276(19)$ & $171(2)$ \\
$\mathrm{O} 1 W-\mathrm{H} 2 W \cdots \mathrm{O} 2^{v}$ & $0.90(3)$ & $2.02(3)$ & $2.906(2)$ & $169(2)$
\end{tabular}

Symmetry codes: (iv) $-x+1 / 2,-y+1 / 2,-z$; (v) $x, y, z-1$.

\section{(CaSr3070)}

\section{Crystal data}

$$
\begin{aligned}
& \mathrm{C}_{14} \mathrm{H}_{14} \mathrm{Ca}_{0.37} \mathrm{O}_{8} \mathrm{Sr}_{0.63} \\
& M_{r}=380.38 \\
& \text { Monoclinic, } C 2 / c \\
& a=16.6319(6) \AA \\
& b=11.4995(4) \AA \\
& c=7.7729(3) \AA \\
& \beta=91.599(4)^{\circ} \\
& V=1486.05(9) \AA^{3} \\
& Z=4
\end{aligned}
$$

\section{Data collection}

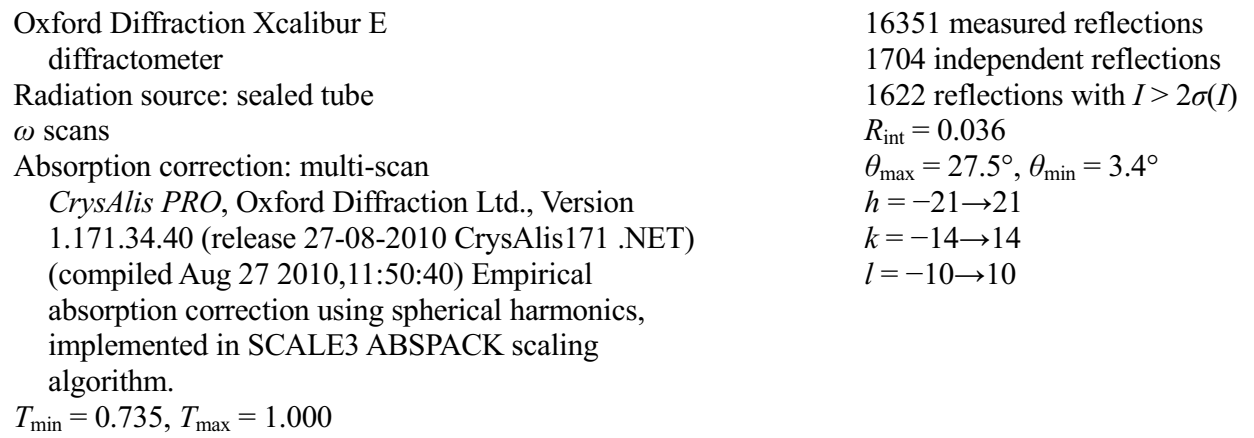

$$
\begin{aligned}
& 16351 \text { measured reflection } \\
& 1704 \text { independent reflect } \\
& 1622 \text { reflections with } I> \\
& R_{\text {int }}=0.036 \\
& \theta_{\max }=27.5^{\circ}, \theta_{\min }=3.4^{\circ} \\
& h=-21 \rightarrow 21 \\
& k=-14 \rightarrow 14 \\
& l=-10 \rightarrow 10
\end{aligned}
$$

16351 measured reflection independent reflections

$F(000)=774$

$D_{\mathrm{x}}=1.700 \mathrm{Mg} \mathrm{m}^{-3}$

Mo $K \alpha$ radiation, $\lambda=0.71073 \AA$

Cell parameters from 10979 reflections

$\theta=3.4-30.4^{\circ}$

$\mu=2.49 \mathrm{~mm}^{-1}$

$T=150 \mathrm{~K}$

Prism, colourless

$0.30 \times 0.25 \times 0.12 \mathrm{~mm}$ 


\section{Refinement}

Refinement on $F^{2}$

Least-squares matrix: full

$R\left[F^{2}>2 \sigma\left(F^{2}\right)\right]=0.021$

$w R\left(F^{2}\right)=0.052$

$S=1.06$

1704 reflections

118 parameters

0 restraints
Hydrogen site location: mixed

$\mathrm{H}$ atoms treated by a mixture of independent and constrained refinement

$w=1 /\left[\sigma^{2}\left(F_{\mathrm{o}}^{2}\right)+(0.0278 P)^{2}+1.2252 P\right]$

where $P=\left(F_{\mathrm{o}}{ }^{2}+2 F_{\mathrm{c}}{ }^{2}\right) / 3$

$(\Delta / \sigma)_{\max }=0.001$

$\Delta \rho_{\max }=0.49 \mathrm{e} \AA^{-3}$

$\Delta \rho_{\min }=-0.25$ e $\AA^{-3}$

\section{Special details}

Geometry. All e.s.d.'s (except the e.s.d. in the dihedral angle between two 1.s. planes) are estimated using the full covariance matrix. The cell e.s.d.'s are taken into account individually in the estimation of e.s.d.'s in distances, angles and torsion angles; correlations between e.s.d.'s in cell parameters are only used when they are defined by crystal symmetry. An approximate (isotropic) treatment of cell e.s.d.'s is used for estimating e.s.d.'s involving l.s. planes.

Fractional atomic coordinates and isotropic or equivalent isotropic displacement parameters $\left(\AA^{2}\right)$

\begin{tabular}{llllll}
\hline & $x$ & $y$ & $z$ & $U_{\text {iso }} * U_{\mathrm{eq}}$ & Occ. $(<1)$ \\
\hline Sr1 & 0.5000 & $0.44518(2)$ & -0.2500 & $0.01297(8)$ & $0.632(4)$ \\
Ca1 & 0.5000 & $0.44518(2)$ & -0.2500 & $0.01297(8)$ & $0.368(4)$ \\
O1 & $0.25649(6)$ & $0.25358(10)$ & $0.26696(14)$ & $0.0226(3)$ & $0.0206(2)$ \\
O2 & $0.37727(6)$ & $0.39842(9)$ & $0.26602(13)$ & $0.0215(3)$ & $0.0283(3)$ \\
O3 & $0.45448(6)$ & $0.39323(9)$ & $0.03965(14)$ & $0.0173(3)$ & $0.0220(3)$ \\
O1W & $0.39587(7)$ & $0.31632(11)$ & $-0.38135(16)$ & $0.026^{*}$ \\
C1 & $0.30065(8)$ & $0.18750(12)$ & $0.15863(18)$ & $0.0255(3)$ & $0.031^{*}$ \\
C2 & $0.27135(9)$ & $0.07781(13)$ & $0.11396(19)$ & $0.0263(4)$ \\
H2 & 0.2210 & 0.0523 & 0.1546 & $0.032^{*}$ \\
C3 & $0.31588(10)$ & $0.00648(14)$ & $0.0105(2)$ & $0.0217(3)$ \\
H3 & 0.2963 & -0.0689 & -0.0180 & $0.026^{*}$ \\
C4 & $0.38909(11)$ & $0.04319(14)$ & $-0.0530(2)$ & $0.0169(3)$ \\
H4 & 0.4197 & -0.0070 & -0.1229 & $0.0167(3)$ \\
C5 & $0.41673(9)$ & $0.15382(13)$ & $-0.01308(19)$ & $0.054(7)^{*}$ \\
H5 & 0.4660 & 0.1800 & -0.0584 & $0.064(8)^{*}$ \\
C6 & $0.37330(8)$ & $0.22746(12)$ & $0.09286(18)$ & $0.043(6)^{*}$ \\
C7 & $0.40328(8)$ & $0.34645(12)$ & $0.13462(18)$ & \\
H1O & $0.2833(15)$ & $0.321(2)$ & $0.284(3)$ & $-0.344(3)$ & \\
H1W & $0.3520(17)$ & $0.292(2)$ & $-0.487(3)$ & & \\
H2W & $0.3891(13)$ & $0.3347(19)$ & & & \\
& & & & &
\end{tabular}

Atomic displacement parameters $\left(\AA^{2}\right)$

\begin{tabular}{lllllll}
\hline & $U^{11}$ & $U^{22}$ & $U^{33}$ & $U^{12}$ & $U^{13}$ & $U^{23}$ \\
\hline Sr1 & $0.01299(12)$ & $0.01185(12)$ & $0.01419(12)$ & 0.000 & $0.00261(7)$ & 0.000 \\
Ca1 & $0.01299(12)$ & $0.01185(12)$ & $0.01419(12)$ & 0.000 & $0.00261(7)$ & 0.000 \\
O1 & $0.0202(5)$ & $0.0221(6)$ & $0.0258(6)$ & $-0.0037(4)$ & $0.0074(4)$ & $-0.0023(4)$ \\
O2 & $0.0208(5)$ & $0.0177(5)$ & $0.0234(5)$ & $-0.0025(4)$ & $0.0042(4)$ & $-0.0034(4)$ \\
O3 & $0.0209(5)$ & $0.0186(5)$ & $0.0254(6)$ & $-0.0041(4)$ & $0.0069(4)$ & $-0.0001(4)$ \\
O1W & $0.0243(6)$ & $0.0364(7)$ & $0.0245(6)$ & $-0.0067(5)$ & $0.0053(5)$ & $0.0037(5)$ \\
C1 & $0.0188(7)$ & $0.0170(7)$ & $0.0162(6)$ & $-0.0010(5)$ & $-0.0003(5)$ & $0.0024(5)$ \\
C2 & $0.0238(7)$ & $0.0211(7)$ & $0.0210(7)$ & $-0.0078(6)$ & $-0.0001(6)$ & $0.0034(6)$ \\
C3 & $0.0373(9)$ & $0.0163(7)$ & $0.0226(8)$ & $-0.0064(6)$ & $-0.0023(6)$ & $0.0001(6)$ \\
C4 & $0.0339(9)$ & $0.0203(8)$ & $0.0248(8)$ & $0.0015(6)$ & $0.0039(6)$ & $-0.0039(6)$
\end{tabular}


supporting information

\begin{tabular}{lllllll} 
C5 & $0.0220(7)$ & $0.0200(7)$ & $0.0231(7)$ & $-0.0001(6)$ & $0.0037(6)$ & $0.0003(6)$ \\
C6 & $0.0173(6)$ & $0.0152(7)$ & $0.0182(7)$ & $-0.0013(5)$ & $0.0000(5)$ & $0.0014(5)$ \\
C7 & $0.0137(6)$ & $0.0170(7)$ & $0.0192(7)$ & $0.0006(5)$ & $0.0003(5)$ & $0.0013(5)$ \\
\hline
\end{tabular}

Geometric parameters $\left(\AA,{ }^{\circ}\right)$

\begin{tabular}{|c|c|c|c|}
\hline $\mathrm{Sr} 1-\mathrm{O}^{\mathrm{i}}$ & $2.4686(10)$ & $\mathrm{O} 3-\mathrm{C} 7$ & $1.2627(17)$ \\
\hline $\mathrm{Sr} 1-\mathrm{O} 3$ & $2.4686(10)$ & $\mathrm{O} 3-\mathrm{Ca}^{\mathrm{ii}}$ & $2.5749(10)$ \\
\hline $\mathrm{Sr} 1-\mathrm{O} 1 \mathrm{~W}^{\mathrm{i}}$ & $2.4777(12)$ & $\mathrm{O} 3-\mathrm{Sr} 1^{\mathrm{ii}}$ & $2.5749(10)$ \\
\hline $\mathrm{Sr} 1-\mathrm{O} 1 \mathrm{~W}$ & $2.4778(12)$ & $\mathrm{O} 1 \mathrm{~W}-\mathrm{H} 1 \mathrm{~W}$ & $0.84(3)$ \\
\hline $\mathrm{Sr} 1-\mathrm{O} 3^{\mathrm{ii}}$ & $2.5749(10)$ & $\mathrm{O} 1 \mathrm{~W}-\mathrm{H} 2 \mathrm{~W}$ & $0.85(2)$ \\
\hline $\mathrm{Sr} 1-\mathrm{O} 3^{\mathrm{iii}}$ & $2.5749(10)$ & $\mathrm{C} 1-\mathrm{C} 2$ & $1.393(2)$ \\
\hline $\mathrm{Sr} 1-\mathrm{O} 2^{\mathrm{ii}}$ & $2.7260(10)$ & $\mathrm{C} 1-\mathrm{C} 6$ & $1.403(2)$ \\
\hline $\mathrm{Sr} 1-\mathrm{O} 2^{\mathrm{iii}}$ & $2.7260(10)$ & $\mathrm{C} 2-\mathrm{C} 3$ & $1.379(2)$ \\
\hline $\mathrm{Sr} 1-\mathrm{C} 7^{\mathrm{ii}}$ & $3.0088(14)$ & $\mathrm{C} 2-\mathrm{H} 2$ & 0.9500 \\
\hline $\mathrm{Sr} 1-\mathrm{C}^{\mathrm{iii}}$ & $3.0088(14)$ & $\mathrm{C} 3-\mathrm{C} 4$ & $1.392(2)$ \\
\hline $\mathrm{Sr} 1-\mathrm{Sr} 1^{\mathrm{ii}}$ & $4.0858(2)$ & $\mathrm{C} 3-\mathrm{H} 3$ & 0.9500 \\
\hline $\mathrm{Sr} 1-\mathrm{Ca}^{\mathrm{ii}}$ & $4.0858(2)$ & $\mathrm{C} 4-\mathrm{C} 5$ & $1.385(2)$ \\
\hline $\mathrm{Sr} 1-\mathrm{H} 2 \mathrm{~W}$ & $2.87(2)$ & $\mathrm{C} 4-\mathrm{H} 4$ & 0.9500 \\
\hline $\mathrm{O} 1-\mathrm{C} 1$ & $1.3639(18)$ & $\mathrm{C} 5-\mathrm{C} 6$ & $1.397(2)$ \\
\hline $\mathrm{O} 1-\mathrm{H} 1 \mathrm{O}$ & $0.90(3)$ & $\mathrm{C} 5-\mathrm{H} 5$ & 0.9500 \\
\hline $\mathrm{O} 2-\mathrm{C} 7$ & $1.2697(18)$ & $\mathrm{C} 6-\mathrm{C} 7$ & $1.4890(19)$ \\
\hline $\mathrm{O} 2-\mathrm{Ca} 1^{\mathrm{ii}}$ & $2.7260(10)$ & $\mathrm{C} 7-\mathrm{Ca} 1^{\mathrm{ii}}$ & $3.0088(14)$ \\
\hline $\mathrm{O} 2-\mathrm{Sr} 1^{\mathrm{ii}}$ & $2.7260(10)$ & $\mathrm{C} 7-\mathrm{Sr} 1^{\mathrm{ii}}$ & $3.0088(14)$ \\
\hline $\mathrm{O} 3{ }^{\mathrm{i}}-\mathrm{Sr} 1-\mathrm{O} 3$ & $151.99(5)$ & $\mathrm{O} 33^{\mathrm{iii}}-\mathrm{Sr} 1-\mathrm{Ca}{ }^{\mathrm{ii}}$ & $111.93(2)$ \\
\hline $\mathrm{O} 3^{\mathrm{i}}-\mathrm{Sr} 1-\mathrm{O} 1 \mathrm{~W}^{\mathrm{i}}$ & $90.14(4)$ & $\mathrm{O} 2^{\mathrm{ii}}-\mathrm{Sr} 1-\mathrm{Ca} 1^{\mathrm{ii}}$ & $81.94(2)$ \\
\hline $\mathrm{O} 3-\mathrm{Sr} 1-\mathrm{O} 1 \mathrm{~W}^{\mathrm{i}}$ & $73.03(4)$ & $\mathrm{O} 2^{\mathrm{iii}}-\mathrm{Sr} 1-\mathrm{Ca}{ }^{\mathrm{ii}}$ & $74.52(2)$ \\
\hline $\mathrm{O} 3{ }^{\mathrm{i}}-\mathrm{Sr} 1-\mathrm{O} 1 \mathrm{~W}$ & $73.03(4)$ & $\mathrm{C}^{\mathrm{ii}}-\mathrm{Sr} 1-\mathrm{Ca}^{\mathrm{ii}}$ & $59.00(3)$ \\
\hline $\mathrm{O} 3-\mathrm{Sr} 1-\mathrm{O} 1 \mathrm{~W}$ & $90.14(4)$ & $\mathrm{C}^{\mathrm{iii}}-\mathrm{Sr} 1-\mathrm{Ca} 1^{\mathrm{ii}}$ & $91.35(3)$ \\
\hline $\mathrm{O} 1 \mathrm{~W}^{\mathrm{i}}-\mathrm{Sr} 1-\mathrm{O} 1 \mathrm{~W}$ & $106.53(6)$ & $\mathrm{Sr} 1^{\mathrm{ii}}-\mathrm{Sr} 1-\mathrm{Ca} 1^{\mathrm{ii}}$ & 0.0 \\
\hline $\mathrm{O} 3^{\mathrm{i}}-\mathrm{Sr} 1-\mathrm{O}^{\mathrm{ii}}$ & $131.42(4)$ & $\mathrm{O} 3{ }^{\mathrm{i}}-\mathrm{Sr} 1-\mathrm{H} 2 \mathrm{~W}$ & $61.0(5)$ \\
\hline $\mathrm{O} 3-\mathrm{Sr} 1-\mathrm{O}^{\mathrm{ii}}$ & $71.81(4)$ & $\mathrm{O} 3-\mathrm{Sr} 1-\mathrm{H} 2 \mathrm{~W}$ & $105.7(5)$ \\
\hline $\mathrm{O} 1 \mathrm{~W}^{\mathrm{i}}-\mathrm{Sr} 1-\mathrm{O} 3^{\mathrm{ii}}$ & $88.87(4)$ & $\mathrm{O} 1 \mathrm{~W}^{\mathrm{i}}-\mathrm{Sr} 1-\mathrm{H} 2 \mathrm{~W}$ & $115.4(5)$ \\
\hline $\mathrm{O} 1 \mathrm{~W}-\mathrm{Sr} 1-\mathrm{O} 3^{\mathrm{ii}}$ & $152.01(4)$ & $\mathrm{O} 1 \mathrm{~W}-\mathrm{Sr} 1-\mathrm{H} 2 \mathrm{~W}$ & $16.4(5)$ \\
\hline $\mathrm{O} 3^{\mathrm{i}}-\mathrm{Sr} 1-\mathrm{O} 3^{\mathrm{iii}}$ & $71.81(4)$ & $\mathrm{O} 3^{\mathrm{ii}}-\mathrm{Sr} 1-\mathrm{H} 2 \mathrm{~W}$ & $154.2(4)$ \\
\hline $\mathrm{O} 3-\mathrm{Sr} 1-\mathrm{O} 3^{\mathrm{iii}}$ & $131.42(4)$ & $\mathrm{O}^{3}{ }^{\mathrm{iii}}-\mathrm{Sr} 1-\mathrm{H} 2 \mathrm{~W}$ & $74.9(5)$ \\
\hline $\mathrm{O} 1 \mathrm{~W}^{\mathrm{i}}-\mathrm{Sr} 1-\mathrm{O} 3^{\mathrm{iii}}$ & $152.01(4)$ & $\mathrm{O} 2^{\mathrm{ii}}-\mathrm{Sr} 1-\mathrm{H} 2 \mathrm{~W}$ & $137.1(5)$ \\
\hline $\mathrm{O} 1 \mathrm{~W}-\mathrm{Sr} 1-\mathrm{O} 3^{\mathrm{iii}}$ & $88.87(4)$ & $\mathrm{O} 2^{\mathrm{iii}-\mathrm{Sr} 1-\mathrm{H} 2 \mathrm{~W}}$ & $81.5(4)$ \\
\hline $\mathrm{O} 3^{\mathrm{ii}}-\mathrm{Sr} 1-\mathrm{O} 3^{\mathrm{iii}}$ & $87.62(5)$ & $\mathrm{C}^{\mathrm{ii}}-\mathrm{Sr} 1-\mathrm{H} 2 \mathrm{~W}$ & $150.9(5)$ \\
\hline $\mathrm{O} 3^{\mathrm{i}}-\mathrm{Sr} 1-\mathrm{O} 2^{\mathrm{ii}}$ & $82.47(3)$ & $\mathrm{C}^{\mathrm{iii}}-\mathrm{Sr} 1-\mathrm{H} 2 \mathrm{~W}$ & $80.3(5)$ \\
\hline $\mathrm{O} 3-\mathrm{Sr} 1-\mathrm{O} 2^{\mathrm{ii}}$ & $116.77(3)$ & $\mathrm{Sr} 1{ }^{\mathrm{ii}}-\mathrm{Sr} 1-\mathrm{H} 2 \mathrm{~W}$ & $137.0(5)$ \\
\hline $\mathrm{O} 1 \mathrm{~W}^{\mathrm{i}}-\mathrm{Sr} 1-\mathrm{O} 2^{\mathrm{ii}}$ & $84.13(4)$ & $\mathrm{Ca} 1^{\mathrm{ii}}-\mathrm{Sr} 1-\mathrm{H} 2 \mathrm{~W}$ & $137.0(5)$ \\
\hline $\mathrm{O} 1 \mathrm{~W}-\mathrm{Sr} 1-\mathrm{O} 2^{\mathrm{ii}}$ & $153.06(4)$ & $\mathrm{C} 1-\mathrm{O} 1-\mathrm{H} 1 \mathrm{O}$ & $107.2(15)$ \\
\hline $\mathrm{O} 3^{\mathrm{ii}}-\mathrm{Sr} 1-\mathrm{O}^{2 \mathrm{ii}}$ & $49.13(3)$ & $\mathrm{C} 7-\mathrm{O} 2-\mathrm{Ca} 1^{\mathrm{ii}}$ & $90.08(8)$ \\
\hline $\mathrm{O} 3^{\mathrm{iii}}-\mathrm{Sr} 1-\mathrm{O} 2^{\mathrm{ii}}$ & $72.67(3)$ & $\mathrm{C} 7-\mathrm{O} 2-\mathrm{Sr}^{\mathrm{ii}}$ & $90.08(8)$ \\
\hline $\mathrm{O} 3^{\mathrm{i}}-\mathrm{Sr} 1-\mathrm{O} 2^{\mathrm{iii}}$ & $116.77(3)$ & $\mathrm{Ca} 1^{\mathrm{ii}}-\mathrm{O} 2-\mathrm{Sr} 1^{\mathrm{ii}}$ & 0.0 \\
\hline $\mathrm{O} 3-\mathrm{Sr} 1-\mathrm{O} 2^{\mathrm{iii}}$ & $82.47(3)$ & $\mathrm{C} 7-\mathrm{O} 3-\mathrm{Sr} 1$ & $149.91(9)$ \\
\hline $\mathrm{O} 1 \mathrm{~W}^{\mathrm{i}}-\mathrm{Sr} 1-\mathrm{O} 2^{\mathrm{iii}}$ & $153.06(4)$ & $\mathrm{C} 7-\mathrm{O} 3-\mathrm{Ca} 1^{\mathrm{ii}}$ & $97.32(8)$ \\
\hline $\mathrm{O} 1 \mathrm{~W}-\mathrm{Sr} 1-\mathrm{O} 2^{\mathrm{iii}}$ & $84.13(4)$ & $\mathrm{Sr} 1-\mathrm{O} 3-\mathrm{Ca} 1^{\mathrm{ii}}$ & 108.2 \\
\hline $\mathrm{O} 3^{\mathrm{ii}}-\mathrm{Sr} 1-\mathrm{O} 2^{\mathrm{iii}}$ & $72.67(3)$ & $\mathrm{C} 7-\mathrm{O} 3-\mathrm{Sr}^{\mathrm{ii}}$ & $97.32(8)$ \\
\hline $\mathrm{O} 3^{\mathrm{iii}}-\mathrm{Sr} 1-\mathrm{O} 2^{\mathrm{iii}}$ & $49.13(3)$ & $\mathrm{Sr} 1-\mathrm{O} 3-\mathrm{Sr} 1^{\mathrm{ii}}$ & $108.19(4)$ \\
\hline
\end{tabular}




\begin{tabular}{|c|c|c|c|}
\hline $\mathrm{O} 2^{\mathrm{ii}}-\mathrm{Sr} 1-\mathrm{O} 2^{\mathrm{iii}}$ & $97.44(4)$ & $\mathrm{Ca} 1^{\mathrm{ii}}-\mathrm{O} 3-\mathrm{Sr}^{\mathrm{ii}}$ & 0.0 \\
\hline $\mathrm{O} 3^{\mathrm{i}}-\mathrm{Sr} 1-\mathrm{C}^{\mathrm{ii}}$ & $106.83(4)$ & $\mathrm{Sr} 1-\mathrm{O} 1 \mathrm{~W}-\mathrm{H} 1 \mathrm{~W}$ & $131.2(18)$ \\
\hline $\mathrm{O} 3-\mathrm{Sr} 1-\mathrm{C}^{\mathrm{ii}}$ & $95.50(4)$ & $\mathrm{Sr} 1-\mathrm{O} 1 \mathrm{~W}-\mathrm{H} 2 \mathrm{~W}$ & $108.7(15)$ \\
\hline $\mathrm{O} 1 \mathrm{~W}^{\mathrm{i}}-\mathrm{Sr} 1-\mathrm{C}^{\mathrm{ii}}$ & $89.53(4)$ & $\mathrm{H} 1 \mathrm{~W}-\mathrm{O} 1 \mathrm{~W}-\mathrm{H} 2 \mathrm{~W}$ & $109(2)$ \\
\hline $\mathrm{O} 1 \mathrm{~W}-\mathrm{Sr} 1-\mathrm{C} 7^{\mathrm{ii}}$ & $163.92(4)$ & $\mathrm{O} 1-\mathrm{C} 1-\mathrm{C} 2$ & $117.89(13)$ \\
\hline $\mathrm{O} 3^{\mathrm{ii}}-\mathrm{Sr} 1-\mathrm{C}^{\mathrm{ii}}$ & $24.60(3)$ & $\mathrm{O} 1-\mathrm{C} 1-\mathrm{C} 6$ & $121.78(13)$ \\
\hline 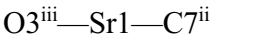 & $76.10(4)$ & $\mathrm{C} 2-\mathrm{C} 1-\mathrm{C} 6$ & $120.32(14)$ \\
\hline $\mathrm{O} 2^{\mathrm{ii}}-\mathrm{Sr} 1-\mathrm{C}^{\mathrm{ii}}$ & $24.96(3)$ & $\mathrm{C} 3-\mathrm{C} 2-\mathrm{C} 1$ & $119.63(14)$ \\
\hline 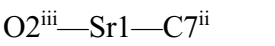 & $81.71(3)$ & $\mathrm{C} 3-\mathrm{C} 2-\mathrm{H} 2$ & 120.2 \\
\hline $\mathrm{O}^{\mathrm{i}}-\mathrm{Sr} 1-\mathrm{C} 7^{\mathrm{iii}}$ & $95.50(4)$ & $\mathrm{C} 1-\mathrm{C} 2-\mathrm{H} 2$ & 120.2 \\
\hline $\mathrm{O} 3-\mathrm{Sr} 1-\mathrm{C} 7^{\mathrm{iii}}$ & $106.83(4)$ & $\mathrm{C} 2-\mathrm{C} 3-\mathrm{C} 4$ & $121.05(14)$ \\
\hline $\mathrm{O} 1 \mathrm{~W}^{\mathrm{i}}-\mathrm{Sr} 1-\mathrm{C} 7^{\mathrm{iii}}$ & $163.92(4)$ & $\mathrm{C} 2-\mathrm{C} 3-\mathrm{H} 3$ & 119.5 \\
\hline $\mathrm{O} 1 \mathrm{~W}-\mathrm{Sr} 1-\mathrm{C}^{\mathrm{iii}}$ & $89.53(4)$ & $\mathrm{C} 4-\mathrm{C} 3-\mathrm{H} 3$ & 119.5 \\
\hline $\mathrm{O} 3^{\mathrm{ii}}-\mathrm{Sr} 1-\mathrm{C} 7^{\mathrm{iii}}$ & $76.10(4)$ & $\mathrm{C} 5-\mathrm{C} 4-\mathrm{C} 3$ & $119.16(15)$ \\
\hline $\mathrm{O} 3^{\mathrm{iii}}-\mathrm{Sr} 1-\mathrm{C} 7^{\mathrm{iii}}$ & $24.60(3)$ & $\mathrm{C} 5-\mathrm{C} 4-\mathrm{H} 4$ & 120.4 \\
\hline $\mathrm{O} 2^{\mathrm{ii}}-\mathrm{Sr} 1-\mathrm{C} 7^{\mathrm{iii}}$ & $81.71(3)$ & $\mathrm{C} 3-\mathrm{C} 4-\mathrm{H} 4$ & 120.4 \\
\hline $\mathrm{O} 2^{\mathrm{iii}}-\mathrm{Sr} 1-\mathrm{C}^{\mathrm{iii}}$ & $24.96(3)$ & $\mathrm{C} 4-\mathrm{C} 5-\mathrm{C} 6$ & $121.02(14)$ \\
\hline $\mathrm{C} 7^{\mathrm{ii}}-\mathrm{Sr} 1-\mathrm{C} 7^{\mathrm{iii}}$ & $74.43(5)$ & $\mathrm{C} 4-\mathrm{C} 5-\mathrm{H} 5$ & 119.5 \\
\hline $\mathrm{O} 3^{\mathrm{i}}-\mathrm{Sr} 1-\mathrm{Sr} 1^{\mathrm{ii}}$ & $161.86(2)$ & $\mathrm{C} 6-\mathrm{C} 5-\mathrm{H} 5$ & 119.5 \\
\hline $\mathrm{O} 3-\mathrm{Sr} 1-\mathrm{Sr} 1^{\mathrm{ii}}$ & $36.78(2)$ & $\mathrm{C} 5-\mathrm{C} 6-\mathrm{C} 1$ & $118.77(13)$ \\
\hline $\mathrm{O} 1 \mathrm{~W}^{\mathrm{i}}-\mathrm{Sr} 1-\mathrm{Sr} 1^{\mathrm{ii}}$ & $79.12(3)$ & $\mathrm{C} 5-\mathrm{C} 6-\mathrm{C} 7$ & $120.68(13)$ \\
\hline $\mathrm{O} 1 \mathrm{~W}-\mathrm{Sr} 1-\mathrm{Sr} 1^{\mathrm{ii}}$ & $123.91(3)$ & $\mathrm{C} 1-\mathrm{C} 6-\mathrm{C} 7$ & $120.55(13)$ \\
\hline $\mathrm{O} 3^{\mathrm{ii}}-\mathrm{Sr} 1-\mathrm{Sr}^{\mathrm{ii}}$ & $35.03(2)$ & $\mathrm{O} 3-\mathrm{C} 7-\mathrm{O} 2$ & $121.35(13)$ \\
\hline $\mathrm{O} 3^{\mathrm{iii}}-\mathrm{Sr} 1-\mathrm{Sr}^{\mathrm{ii}}$ & $111.93(2)$ & $\mathrm{O} 3-\mathrm{C} 7-\mathrm{C} 6$ & $119.42(13)$ \\
\hline $\mathrm{O} 2^{\mathrm{ii}}-\mathrm{Sr} 1-\mathrm{Sr} 1^{\mathrm{ii}}$ & $81.94(2)$ & $\mathrm{O} 2-\mathrm{C} 7-\mathrm{C} 6$ & $119.23(12)$ \\
\hline $\mathrm{O} 2^{\mathrm{iii}}-\mathrm{Sr} 1-\mathrm{Sr} 1^{\mathrm{ii}}$ & $74.52(2)$ & $\mathrm{O} 3-\mathrm{C} 7-\mathrm{Ca}^{\mathrm{ii}}$ & $58.08(7)$ \\
\hline $\mathrm{C}^{\mathrm{ii}}-\mathrm{Sr} 1-\mathrm{Sr} 1^{\mathrm{ii}}$ & $59.00(3)$ & $\mathrm{O} 2-\mathrm{C} 7-\mathrm{Ca}^{\mathrm{ii}}$ & $64.96(7)$ \\
\hline $\mathrm{C} 7^{\mathrm{iii}}-\mathrm{Sr} 1-\mathrm{Sr} 1^{\mathrm{ii}}$ & $91.35(3)$ & $\mathrm{C} 6-\mathrm{C} 7-\mathrm{Ca} 1^{\mathrm{ii}}$ & $165.88(9)$ \\
\hline $\mathrm{O} 3^{\mathrm{i}}-\mathrm{Sr} 1-\mathrm{Ca} 1^{\mathrm{ii}}$ & $161.86(2)$ & $\mathrm{O} 3-\mathrm{C} 7-\mathrm{Sr} 1^{\mathrm{ii}}$ & $58.08(7)$ \\
\hline $\mathrm{O} 3-\mathrm{Sr} 1-\mathrm{Ca} 1^{\mathrm{ii}}$ & $36.78(2)$ & $\mathrm{O} 2-\mathrm{C} 7-\mathrm{Sr}^{\mathrm{ii}}$ & $64.96(7)$ \\
\hline $\mathrm{O} 1 \mathrm{~W}^{\mathrm{i}}-\mathrm{Sr} 1-\mathrm{Ca}^{\mathrm{ii}}$ & $79.12(3)$ & $\mathrm{C} 6-\mathrm{C} 7-\mathrm{Sr} 1^{\mathrm{ii}}$ & $165.88(9)$ \\
\hline $\mathrm{O} 1 \mathrm{~W}-\mathrm{Sr} 1-\mathrm{Ca} 1^{\mathrm{ii}}$ & $123.91(3)$ & $\mathrm{Ca} 1^{\mathrm{ii}}-\mathrm{C} 7-\mathrm{Sr} 1^{\mathrm{ii}}$ & 0.0 \\
\hline $\mathrm{O} 3^{\mathrm{ii}}-\mathrm{Sr} 1-\mathrm{Ca}^{\mathrm{ii}}$ & $35.03(2)$ & & \\
\hline $\mathrm{O} 1-\mathrm{C} 1-\mathrm{C} 2-\mathrm{C} 3$ & $-177.33(13)$ & $\mathrm{Sr}^{\mathrm{ii}}-\mathrm{O} 3-\mathrm{C} 7-\mathrm{Ca}^{\mathrm{ii}}$ & 0.0 \\
\hline $\mathrm{C} 6-\mathrm{C} 1-\mathrm{C} 2-\mathrm{C} 3$ & $2.8(2)$ & $\mathrm{Sr} 1-\mathrm{O} 3-\mathrm{C} 7-\mathrm{Sr} 1^{\mathrm{ii}}$ & $-148.2(2)$ \\
\hline $\mathrm{C} 1-\mathrm{C} 2-\mathrm{C} 3-\mathrm{C} 4$ & $-1.3(2)$ & $\mathrm{Ca} 1^{\mathrm{ii}}-\mathrm{O} 3-\mathrm{C} 7-\mathrm{Sr} 1^{\mathrm{ii}}$ & 0.0 \\
\hline $\mathrm{C} 2-\mathrm{C} 3-\mathrm{C} 4-\mathrm{C} 5$ & $-0.9(2)$ & $\mathrm{Ca} 1^{\mathrm{ii}}-\mathrm{O} 2-\mathrm{C} 7-\mathrm{O} 3$ & $-14.58(13)$ \\
\hline $\mathrm{C} 3-\mathrm{C} 4-\mathrm{C} 5-\mathrm{C} 6$ & $1.6(2)$ & $\mathrm{Sr} 1^{\mathrm{ii}}-\mathrm{O} 2-\mathrm{C} 7-\mathrm{O} 3$ & $-14.58(13)$ \\
\hline $\mathrm{C} 4-\mathrm{C} 5-\mathrm{C} 6-\mathrm{C} 1$ & $-0.1(2)$ & $\mathrm{Ca} 1{ }^{\mathrm{ii}}-\mathrm{O} 2-\mathrm{C} 7-\mathrm{C} 6$ & $164.83(11)$ \\
\hline $\mathrm{C} 4-\mathrm{C} 5-\mathrm{C} 6-\mathrm{C} 7$ & $-179.76(14)$ & $\mathrm{Sr} 1^{\mathrm{ii}}-\mathrm{O} 2-\mathrm{C} 7-\mathrm{C} 6$ & $164.83(11)$ \\
\hline $\mathrm{O} 1-\mathrm{C} 1-\mathrm{C} 6-\mathrm{C} 5$ & $177.99(13)$ & $\mathrm{Sr} 1^{\mathrm{ii}}-\mathrm{O} 2-\mathrm{C} 7-\mathrm{Ca} 1^{\mathrm{ii}}$ & 0.0 \\
\hline $\mathrm{C} 2-\mathrm{C} 1-\mathrm{C} 6-\mathrm{C} 5$ & $-2.1(2)$ & $\mathrm{Ca} 1^{\mathrm{ii}}-\mathrm{O} 2-\mathrm{C} 7-\mathrm{Sr} 1^{\mathrm{ii}}$ & 0.0 \\
\hline $\mathrm{O} 1-\mathrm{C} 1-\mathrm{C} 6-\mathrm{C} 7$ & $-2.3(2)$ & $\mathrm{C} 5-\mathrm{C} 6-\mathrm{C} 7-\mathrm{O} 3$ & $19.7(2)$ \\
\hline $\mathrm{C} 2-\mathrm{C} 1-\mathrm{C} 6-\mathrm{C} 7$ & $177.57(13)$ & $\mathrm{C} 1-\mathrm{C} 6-\mathrm{C} 7-\mathrm{O} 3$ & $-160.00(13)$ \\
\hline $\mathrm{Sr} 1-\mathrm{O} 3-\mathrm{C} 7-\mathrm{O} 2$ & $-132.57(16)$ & $\mathrm{C} 5-\mathrm{C} 6-\mathrm{C} 7-\mathrm{O} 2$ & $-159.73(14)$ \\
\hline $\mathrm{Ca}{ }^{\mathrm{ii}}-\mathrm{O} 3-\mathrm{C} 7-\mathrm{O} 2$ & $15.58(14)$ & $\mathrm{C} 1-\mathrm{C} 6-\mathrm{C} 7-\mathrm{O} 2$ & $20.6(2)$ \\
\hline $\mathrm{Sr}^{\mathrm{ii}}-\mathrm{O} 3-\mathrm{C} 7-\mathrm{O} 2$ & $15.58(14)$ & $\mathrm{C} 5-\mathrm{C} 6-\mathrm{C} 7-\mathrm{Ca}^{\mathrm{ii}}$ & $-56.1(5)$ \\
\hline $\mathrm{Sr} 1-\mathrm{O} 3-\mathrm{C} 7-\mathrm{C} 6$ & $48.0(2)$ & $\mathrm{C} 1-\mathrm{C} 6-\mathrm{C} 7-\mathrm{Ca}^{\mathrm{ii}}$ & $124.2(4)$ \\
\hline $\mathrm{Ca}{ }^{\mathrm{ii}}-\mathrm{O} 3-\mathrm{C} 7-\mathrm{C} 6$ & $-163.82(10)$ & $\mathrm{C} 5-\mathrm{C} 6-\mathrm{C} 7-\mathrm{Sr}^{\mathrm{ii}}$ & $-56.1(5)$ \\
\hline
\end{tabular}


$\mathrm{Sr} 1$ ii $-\mathrm{O} 3-\mathrm{C} 7-\mathrm{C} 6$

$\mathrm{Sr} 1-\mathrm{O} 3-\mathrm{C} 7-\mathrm{Ca}^{\mathrm{ii}}$
$-163.82(10)$

$-148.2(2)$

$124.2(4)$

Symmetry codes: (i) $-x+1, y,-z-1 / 2$; (ii) $-x+1,-y+1,-z$; (iii) $x,-y+1, z-1 / 2$.

Hydrogen-bond geometry $\left(A,{ }^{\circ}\right)$

\begin{tabular}{lllll}
\hline$D-\mathrm{H} \cdots A$ & $D-\mathrm{H}$ & $\mathrm{H} \cdots A$ & $D \cdots A$ & $D-\mathrm{H} \cdots A$ \\
\hline $\mathrm{O} 1-\mathrm{H} 1 O \cdots \mathrm{O} 2$ & $0.90(3)$ & $1.81(3)$ & $2.6096(15)$ & $147(2)$ \\
$\mathrm{O} 1 W-\mathrm{H} 1 W \cdots \mathrm{O} 1^{\mathrm{iv}}$ & $0.84(3)$ & $1.99(3)$ & $2.8255(16)$ & $175(3)$ \\
$\mathrm{O} 1 W-\mathrm{H} 2 W \cdots \mathrm{O} 2^{\mathrm{v}}$ & $0.85(2)$ & $2.06(2)$ & $2.9074(17)$ & $173(2)$ \\
\hline
\end{tabular}

Symmetry codes: (iv) $-x+1 / 2,-y+1 / 2,-z$; (v) $x, y, z-1$.

\section{(CaSr2080)}

\section{Crystal data}

$\mathrm{C}_{14} \mathrm{H}_{14} \mathrm{Ca}_{0.21} \mathrm{O}_{8} \mathrm{Sr}_{0.79}$

$M_{r}=387.85$

Monoclinic, $C 2 / c$

$a=16.6650(11) \AA$

$b=11.4816(7) \AA$

$c=7.8105(5) \AA$

$\beta=91.576(6)^{\circ}$

$V=1493.90(16) \AA^{3}$

$Z=4$

\section{Data collection}

Oxford Diffraction Xcalibur E diffractometer

Radiation source: sealed tube $\omega$ scans
$F(000)=785$

$D_{\mathrm{x}}=1.724 \mathrm{Mg} \mathrm{m}^{-3}$

Mo $K \alpha$ radiation, $\lambda=0.71073 \AA$

Cell parameters from 4057 reflections

$\theta=3.4-30.1^{\circ}$

$\mu=2.98 \mathrm{~mm}^{-1}$

$T=150 \mathrm{~K}$

Cut prism, colourless

$0.26 \times 0.25 \times 0.15 \mathrm{~mm}$

\section{Refinement}

Refinement on $F^{2}$

Least-squares matrix: full

$R\left[F^{2}>2 \sigma\left(F^{2}\right)\right]=0.024$

$w R\left(F^{2}\right)=0.054$

$S=1.06$

1716 reflections

118 parameters

0 restraints
Absorption correction: multi-scan

CrysAlis PRO, Oxford Diffraction Ltd., Version

1.171.34.40 (release 27-08-2010 CrysAlis171 .NET)

(compiled Aug 27 2010,11:50:40) Empirical

absorption correction using spherical harmonics, implemented in SCALE3 ABSPACK scaling algorithm.

$T_{\min }=0.896, T_{\max }=1.000$

6802 measured reflections

1716 independent reflections

1594 reflections with $I>2 \sigma(I)$

$R_{\text {int }}=0.041$

$\theta_{\max }=27.5^{\circ}, \theta_{\min }=3.4^{\circ}$

$h=-21 \rightarrow 21$

$k=-14 \rightarrow 14$

$l=-10 \rightarrow 10$

Hydrogen site location: mixed

$\mathrm{H}$ atoms treated by a mixture of independent and constrained refinement

$w=1 /\left[\sigma^{2}\left(F_{\mathrm{o}}^{2}\right)+(0.0229 P)^{2}\right]$

where $P=\left(F_{\mathrm{o}}^{2}+2 F_{\mathrm{c}}^{2}\right) / 3$

$(\Delta / \sigma)_{\max }<0.001$

$\Delta \rho_{\max }=0.31 \mathrm{e} \AA^{-3}$

$\Delta \rho_{\min }=-0.32$ e $\AA^{-3}$ 


\section{Special details}

Geometry. All e.s.d.'s (except the e.s.d. in the dihedral angle between two 1.s. planes) are estimated using the full covariance matrix. The cell e.s.d.'s are taken into account individually in the estimation of e.s.d.'s in distances, angles and torsion angles; correlations between e.s.d.'s in cell parameters are only used when they are defined by crystal symmetry. An approximate (isotropic) treatment of cell e.s.d.'s is used for estimating e.s.d.'s involving l.s. planes.

Fractional atomic coordinates and isotropic or equivalent isotropic displacement parameters $\left(\AA^{2}\right)$

\begin{tabular}{llllll}
\hline & $x$ & $y$ & $z$ & $U_{\text {iso }} * U_{\mathrm{eq}}$ & Occ. $(<1)$ \\
\hline Sr1 & 0.5000 & $0.44477(2)$ & -0.2500 & $0.01095(9)$ & $0.789(4)$ \\
Ca1 & 0.5000 & $0.44477(2)$ & -0.2500 & $0.01095(9)$ & $0.211(4)$ \\
O1 & $0.25656(7)$ & $0.25362(12)$ & $0.26623(17)$ & $0.0208(3)$ & $0.0186(3)$ \\
O2 & $0.37707(7)$ & $0.39785(11)$ & $0.26542(16)$ & $0.0187(3)$ & $0.0262(4)$ \\
O3 & $0.45435(7)$ & $0.39201(11)$ & $0.04011(16)$ & $0.0160(4)$ \\
O1W & $0.39514(8)$ & $0.31499(13)$ & $-0.38344(19)$ & $0.0199(4)$ \\
C1 & $0.30041(10)$ & $0.18727(15)$ & $0.1585(2)$ & $0.024^{*}$ & $0.0239(4)$ \\
C2 & $0.27070(11)$ & $0.07764(15)$ & $0.1144(2)$ & $0.029 *$ \\
H2 & 0.2205 & 0.0522 & 0.1554 & $0.0248(5)$ \\
C3 & $0.31486(12)$ & $0.00625(16)$ & $0.0105(2)$ & $0.030^{*}$ \\
H3 & 0.2949 & -0.0689 & -0.0187 & $0.0201(4)$ \\
C4 & $0.38783(12)$ & $0.04244(16)$ & $-0.0520(3)$ & $0.024 *$ \\
H4 & 0.4183 & -0.0081 & -0.1213 & $0.0155(4)$ \\
C5 & $0.41577(11)$ & $0.15290(15)$ & $-0.0124(2)$ & $0.0146(4)$ \\
H5 & 0.4650 & 0.1788 & -0.0578 & $0.064(9)^{*}$ \\
C6 & $0.37279(10)$ & $0.22681(15)$ & $0.0933(2)$ & $0.060(8)^{*}$ \\
C7 & $0.40303(10)$ & $0.34547(15)$ & $0.1344(2)$ & $0.047(8)^{*}$ \\
H1O & $0.2797(15)$ & $0.323(2)$ & $0.280(4)$ & \\
H1W & $0.3496(16)$ & $0.288(2)$ & $-0.344(4)$ & $-0.487(3)$ & \\
H2W & $0.3899(14)$ & $0.338(2)$ & & &
\end{tabular}

Atomic displacement parameters $\left(\AA^{2}\right)$

\begin{tabular}{lllllll}
\hline & $U^{11}$ & $U^{22}$ & $U^{33}$ & $U^{12}$ & $U^{13}$ & $U^{23}$ \\
\hline Sr1 & $0.01062(14)$ & $0.01113(14)$ & $0.01121(13)$ & 0.000 & $0.00220(9)$ & 0.000 \\
Ca1 & $0.01062(14)$ & $0.01113(14)$ & $0.01121(13)$ & 0.000 & $0.00220(9)$ & 0.000 \\
O1 & $0.0178(7)$ & $0.0216(7)$ & $0.0234(7)$ & $-0.0037(5)$ & $0.0074(5)$ & $-0.0023(6)$ \\
O2 & $0.0183(7)$ & $0.0181(7)$ & $0.0197(7)$ & $-0.0025(5)$ & $0.0047(5)$ & $-0.0036(5)$ \\
O3 & $0.0169(6)$ & $0.0196(7)$ & $0.0198(7)$ & $-0.0055(5)$ & $0.0052(5)$ & $0.0007(5)$ \\
O1W & $0.0222(8)$ & $0.0356(9)$ & $0.0210(8)$ & $-0.0090(6)$ & $0.0045(6)$ & $0.0022(6)$ \\
C1 & $0.0177(9)$ & $0.0176(9)$ & $0.0126(9)$ & $0.0008(7)$ & $-0.0001(7)$ & $0.0026(7)$ \\
C2 & $0.0211(10)$ & $0.0204(10)$ & $0.0181(9)$ & $-0.0069(7)$ & $-0.0016(7)$ & $0.0025(7)$ \\
C3 & $0.0366(11)$ & $0.0154(9)$ & $0.0194(10)$ & $-0.0079(8)$ & $-0.0029(8)$ & $0.0001(8)$ \\
C4 & $0.0328(12)$ & $0.0194(10)$ & $0.0223(10)$ & $0.0027(8)$ & $0.0031(8)$ & $-0.0039(8)$ \\
C5 & $0.0197(10)$ & $0.0211(10)$ & $0.0194(10)$ & $0.0006(7)$ & $0.0026(7)$ & $-0.0001(8)$ \\
C6 & $0.0165(9)$ & $0.0159(9)$ & $0.0142(9)$ & $-0.0018(7)$ & $-0.0010(7)$ & $0.0001(7)$ \\
C7 & $0.0112(8)$ & $0.0170(9)$ & $0.0153(9)$ & $0.0008(7)$ & $-0.0012(6)$ & $0.0020(7)$ \\
\end{tabular}

Geometric parameters $\left(A,{ }^{\circ}\right)$

\begin{tabular}{lllr}
\hline $\mathrm{Sr} 1-\mathrm{O} 3^{\mathrm{i}}$ & $2.4849(12)$ & $\mathrm{O} 3-\mathrm{C} 7$ & $1.2623(19)$ \\
$\mathrm{Sr} 1-\mathrm{O} 3$ & $2.4849(12)$ & $\mathrm{O} 3-\mathrm{Ca} 1^{\mathrm{ii}}$ & $2.5903(12)$ \\
$\mathrm{Sr} 1-\mathrm{O} 1 W^{\mathrm{i}}$ & $2.5020(14)$ & $\mathrm{O} 3-\mathrm{Sr} 1^{\mathrm{ii}}$ & $2.5903(12)$
\end{tabular}




\begin{tabular}{|c|c|c|c|}
\hline $\mathrm{Sr} 1-\mathrm{O} 1 \mathrm{~W}$ & $2.5020(14)$ & $\mathrm{O} 1 \mathrm{~W}-\mathrm{H} 1 \mathrm{~W}$ & $0.88(3)$ \\
\hline $\mathrm{Sr} 1-\mathrm{O} 3^{\mathrm{ii}}$ & $2.5902(12)$ & $\mathrm{O} 1 \mathrm{~W}-\mathrm{H} 2 \mathrm{~W}$ & $0.85(3)$ \\
\hline $\mathrm{Sr} 1-\mathrm{O} 3^{\mathrm{iii}}$ & $2.5902(12)$ & $\mathrm{C} 1-\mathrm{C} 2$ & $1.393(2)$ \\
\hline $\mathrm{Sr} 1-\mathrm{O} 2^{\mathrm{ii}}$ & $2.7368(12)$ & $\mathrm{C} 1-\mathrm{C} 6$ & $1.398(2)$ \\
\hline $\mathrm{Sr} 1-\mathrm{O} 2^{\mathrm{iii}}$ & $2.7368(12)$ & $\mathrm{C} 2-\mathrm{C} 3$ & $1.380(3)$ \\
\hline $\mathrm{Sr} 1-\mathrm{C} 7^{\mathrm{ii}}$ & 3.0245 (17) & $\mathrm{C} 2-\mathrm{H} 2$ & 0.9500 \\
\hline $\mathrm{Sr} 1-\mathrm{C}^{\mathrm{iii}}$ & $3.0245(17)$ & $\mathrm{C} 3-\mathrm{C} 4$ & $1.387(3)$ \\
\hline $\mathrm{Sr} 1-\mathrm{Sr} 1^{\mathrm{ii}}$ & $4.1061(3)$ & $\mathrm{C} 3-\mathrm{H} 3$ & 0.9500 \\
\hline $\mathrm{Sr} 1-\mathrm{Ca} 1^{\mathrm{ii}}$ & $4.1061(3)$ & $\mathrm{C} 4-\mathrm{C} 5$ & $1.383(2)$ \\
\hline $\mathrm{Sr} 1-\mathrm{H} 2 \mathrm{~W}$ & $2.85(3)$ & $\mathrm{C} 4-\mathrm{H} 4$ & 0.9500 \\
\hline $\mathrm{O} 1-\mathrm{C} 1$ & $1.362(2)$ & $\mathrm{C} 5-\mathrm{C} 6$ & $1.395(2)$ \\
\hline $\mathrm{O} 1-\mathrm{H} 1 \mathrm{O}$ & $0.89(3)$ & $\mathrm{C} 5-\mathrm{H} 5$ & 0.9500 \\
\hline $\mathrm{O} 2-\mathrm{C} 7$ & $1.273(2)$ & $\mathrm{C} 6-\mathrm{C} 7$ & $1.485(2)$ \\
\hline $\mathrm{O} 2-\mathrm{Ca} 1^{\mathrm{ii}}$ & $2.7368(12)$ & $\mathrm{C} 7-\mathrm{Ca}^{\mathrm{ii}}$ & $3.0245(17)$ \\
\hline $\mathrm{O} 2-\mathrm{Sr} 1^{\mathrm{ii}}$ & $2.7368(12)$ & $\mathrm{C} 7-\mathrm{Sr}^{\mathrm{ii}}$ & $3.0245(17)$ \\
\hline $\mathrm{O} 3{ }^{\mathrm{i}}-\mathrm{Sr} 1-\mathrm{O} 3$ & $151.79(6)$ & $\mathrm{O} 3^{\mathrm{iii}}-\mathrm{Sr} 1-\mathrm{Ca} 1^{\mathrm{ii}}$ & $111.76(3)$ \\
\hline $\mathrm{O} 3^{\mathrm{i}}-\mathrm{Sr} 1-\mathrm{O} 1 \mathrm{~W}^{\mathrm{i}}$ & $90.39(4)$ & $\mathrm{O} 2^{\mathrm{ii}}-\mathrm{Sr} 1-\mathrm{Ca} 1^{\mathrm{ii}}$ & $81.81(3)$ \\
\hline $\mathrm{O} 3-\mathrm{Sr} 1-\mathrm{O} 1 \mathrm{~W}^{\mathrm{i}}$ & $72.71(4)$ & $\mathrm{O} 2^{\mathrm{iii}}-\mathrm{Sr} 1-\mathrm{Ca} 1^{\mathrm{ii}}$ & $74.61(3)$ \\
\hline $\mathrm{O}^{\mathrm{i}}-\mathrm{Sr} 1-\mathrm{O} 1 \mathrm{~W}$ & $72.71(4)$ & $\mathrm{C} 7^{\mathrm{ii}}-\mathrm{Sr} 1-\mathrm{Ca} 1^{\mathrm{ii}}$ & $58.93(3)$ \\
\hline $\mathrm{O} 3-\mathrm{Sr} 1-\mathrm{O} 1 \mathrm{~W}$ & $90.39(4)$ & $\mathrm{C} 7^{\mathrm{iii}}-\mathrm{Sr} 1-\mathrm{Ca} 1^{\mathrm{ii}}$ & $91.38(3)$ \\
\hline $\mathrm{O}_{1} \mathrm{~W}^{\mathrm{i}}-\mathrm{Sr} 1-\mathrm{O} 1 \mathrm{~W}$ & $106.90(7)$ & $\mathrm{Sr} 1^{\mathrm{ii}}-\mathrm{Sr} 1-\mathrm{Ca} 1^{\mathrm{ii}}$ & 0.0 \\
\hline $\mathrm{O} 3^{\mathrm{i}}-\mathrm{Sr} 1-\mathrm{O} 3^{\mathrm{ii}}$ & $131.42(4)$ & $\mathrm{O} 3^{\mathrm{i}}-\mathrm{Sr} 1-\mathrm{H} 2 \mathrm{~W}$ & $60.7(5)$ \\
\hline $\mathrm{O} 3-\mathrm{Sr} 1-\mathrm{O}^{\mathrm{ii}}$ & $72.01(4)$ & $\mathrm{O} 3-\mathrm{Sr} 1-\mathrm{H} 2 \mathrm{~W}$ & $106.2(5)$ \\
\hline $\mathrm{O} 1 \mathrm{~W}^{\mathrm{i}}-\mathrm{Sr} 1-\mathrm{O} 3^{\mathrm{ii}}$ & $88.72(5)$ & $\mathrm{O} 1 \mathrm{~W}^{\mathrm{i}}-\mathrm{Sr} 1-\mathrm{H} 2 \mathrm{~W}$ & $116.3(5)$ \\
\hline $\mathrm{O} 1 \mathrm{~W}-\mathrm{Sr} 1-\mathrm{O} 3^{\mathrm{ii}}$ & $152.14(4)$ & $\mathrm{O} 1 \mathrm{~W}-\mathrm{Sr} 1-\mathrm{H} 2 \mathrm{~W}$ & $16.7(5)$ \\
\hline $\mathrm{O} 3^{\mathrm{i}}-\mathrm{Sr} 1-\mathrm{O} 3^{\mathrm{iii}}$ & $72.01(4)$ & $\mathrm{O} 3{ }^{\mathrm{ii}}-\mathrm{Sr} 1-\mathrm{H} 2 \mathrm{~W}$ & $153.7(5)$ \\
\hline $\mathrm{O} 3-\mathrm{Sr} 1-\mathrm{O} 3^{\mathrm{iii}}$ & $131.42(4)$ & $\mathrm{O} 33^{\mathrm{iii}}-\mathrm{Sr} 1-\mathrm{H} 2 \mathrm{~W}$ & $74.3(5)$ \\
\hline $\mathrm{O} 1 \mathrm{~W}^{\mathrm{i}}-\mathrm{Sr} 1-\mathrm{O}^{\mathrm{iii}}$ & $152.14(4)$ & $\mathrm{O} 2 \mathrm{ii}-\mathrm{Sr} 1-\mathrm{H} 2 \mathrm{~W}$ & $136.6(5)$ \\
\hline $\mathrm{O} 1 \mathrm{~W}-\mathrm{Sr} 1-\mathrm{O} 3^{\mathrm{iii}}$ & $88.72(5)$ & $\mathrm{O} 2{ }^{\mathrm{iii}}-\mathrm{Sr} 1-\mathrm{H} 2 \mathrm{~W}$ & $81.1(5)$ \\
\hline $\mathrm{O} 3^{\mathrm{ii}}-\mathrm{Sr} 1-\mathrm{O} 3^{\mathrm{iii}}$ & $87.32(6)$ & $\mathrm{C}^{\mathrm{ii}}-\mathrm{Sr} 1-\mathrm{H} 2 \mathrm{~W}$ & $150.2(5)$ \\
\hline $\mathrm{O} 3^{\mathrm{i}}-\mathrm{Sr} 1-\mathrm{O} 2^{\mathrm{ii}}$ & $82.68(4)$ & $\mathrm{C} 7{ }^{\mathrm{iii}}-\mathrm{Sr} 1-\mathrm{H} 2 \mathrm{~W}$ & $79.7(5)$ \\
\hline $\mathrm{O} 3-\mathrm{Sr} 1-\mathrm{O} 2^{\mathrm{ii}}$ & $116.70(4)$ & $\mathrm{Sr} 1{ }^{\mathrm{ii}}-\mathrm{Sr} 1-\mathrm{H} 2 \mathrm{~W}$ & $137.2(5)$ \\
\hline $\mathrm{O} 1 \mathrm{~W}^{\mathrm{i}}-\mathrm{Sr} 1-\mathrm{O} 2^{\mathrm{ii}}$ & $84.06(4)$ & $\mathrm{Ca} 1^{\mathrm{ii}}-\mathrm{Sr} 1-\mathrm{H} 2 \mathrm{~W}$ & $137.2(5)$ \\
\hline $\mathrm{O} 1 \mathrm{~W}-\mathrm{Sr} 1-\mathrm{O} 2^{\mathrm{ii}}$ & $152.87(4)$ & $\mathrm{C} 1-\mathrm{O} 1-\mathrm{H} 1 \mathrm{O}$ & $109.9(16)$ \\
\hline $\mathrm{O} 3^{\mathrm{ii}}-\mathrm{Sr} 1-\mathrm{O} 2^{\mathrm{ii}}$ & $48.93(3)$ & $\mathrm{C} 7-\mathrm{O} 2-\mathrm{Ca} 1^{\mathrm{ii}}$ & $90.30(9)$ \\
\hline $\mathrm{O} 3^{\mathrm{iii}}-\mathrm{Sr} 1-\mathrm{O} 2^{\mathrm{ii}}$ & $72.64(4)$ & $\mathrm{C} 7-\mathrm{O} 2-\mathrm{Sr} 1^{\mathrm{ii}}$ & $90.30(9)$ \\
\hline $\mathrm{O} 3^{\mathrm{i}}-\mathrm{Sr} 1-\mathrm{O} 2^{\mathrm{iii}}$ & $116.70(4)$ & $\mathrm{Ca} 1^{\mathrm{ii}}-\mathrm{O} 2-\mathrm{Sr} 1^{\mathrm{ii}}$ & 0.0 \\
\hline $\mathrm{O} 3-\mathrm{Sr} 1-\mathrm{O} 2^{\mathrm{iii}}$ & $82.68(4)$ & $\mathrm{C} 7-\mathrm{O} 3-\mathrm{Sr} 1$ & $149.79(11)$ \\
\hline $\mathrm{O} 1 \mathrm{~W}^{\mathrm{i}}-\mathrm{Sr} 1-\mathrm{O} 2^{\mathrm{iii}}$ & $152.87(4)$ & $\mathrm{C} 7-\mathrm{O} 3-\mathrm{Ca} 1^{\mathrm{ii}}$ & $97.42(10)$ \\
\hline $\mathrm{O} 1 \mathrm{~W}-\mathrm{Sr} 1-\mathrm{O} 2^{\mathrm{iii}}$ & $84.06(4)$ & $\mathrm{Sr} 1-\mathrm{O} 3-\mathrm{Ca}^{\mathrm{ii}}$ & 108.0 \\
\hline $\mathrm{O} 3^{\mathrm{ii}}-\mathrm{Sr} 1-\mathrm{O} 2^{\mathrm{iii}}$ & $72.64(4)$ & $\mathrm{C} 7-\mathrm{O} 3-\mathrm{Sr} 1^{\mathrm{ii}}$ & $97.42(10)$ \\
\hline $\mathrm{O} 3^{\mathrm{iii}}-\mathrm{Sr} 1-\mathrm{O} 2^{\mathrm{iii}}$ & $48.93(3)$ & $\mathrm{Sr} 1-\mathrm{O} 3-\mathrm{Sr} 1^{\mathrm{ii}}$ & $107.99(4)$ \\
\hline $\mathrm{O} 2^{\mathrm{ii}}-\mathrm{Sr} 1-\mathrm{O} 2^{\mathrm{iii}}$ & $97.36(5)$ & $\mathrm{Ca} 1^{\mathrm{ii}}-\mathrm{O} 3-\mathrm{Sr} 1^{\mathrm{ii}}$ & 0.0 \\
\hline $\mathrm{O} 3^{\mathrm{i}}-\mathrm{Sr} 1-\mathrm{C}^{\mathrm{ii}}$ & $106.98(4)$ & $\mathrm{Sr} 1-\mathrm{O} 1 \mathrm{~W}-\mathrm{H} 1 \mathrm{~W}$ & $131.3(17)$ \\
\hline $\mathrm{O} 3-\mathrm{Sr} 1-\mathrm{C}^{\mathrm{ii}}$ & $95.52(4)$ & $\mathrm{Sr} 1-\mathrm{O} 1 \mathrm{~W}-\mathrm{H} 2 \mathrm{~W}$ & $105.4(17)$ \\
\hline $\mathrm{O} 1 \mathrm{~W}^{\mathrm{i}}-\mathrm{Sr} 1-\mathrm{C}^{\mathrm{ii}}$ & $89.34(5)$ & $\mathrm{H} 1 \mathrm{~W}-\mathrm{O} 1 \mathrm{~W}-\mathrm{H} 2 \mathrm{~W}$ & $112(2)$ \\
\hline $\mathrm{O} 1 \mathrm{~W}-\mathrm{Sr} 1-\mathrm{C} 7^{\mathrm{ii}}$ & $163.73(5)$ & $\mathrm{O} 1-\mathrm{C} 1-\mathrm{C} 2$ & $117.73(15)$ \\
\hline $\mathrm{O} 3^{\mathrm{ii}}-\mathrm{Sr} 1-\mathrm{C}^{\mathrm{ii}}$ & $24.45(4)$ & $\mathrm{O} 1-\mathrm{C} 1-\mathrm{C} 6$ & $121.76(15)$ \\
\hline $\mathrm{O} 3^{\mathrm{iii}}-\mathrm{Sr} 1-\mathrm{C}^{\mathrm{ii}}$ & $76.00(4)$ & $\mathrm{C} 2-\mathrm{C} 1-\mathrm{C} 6$ & $120.51(16)$ \\
\hline $\mathrm{O} 2^{\mathrm{ii}}-\mathrm{Sr} 1-\mathrm{C} 7^{\mathrm{ii}}$ & $24.90(4)$ & $\mathrm{C} 3-\mathrm{C} 2-\mathrm{C} 1$ & $119.39(17)$ \\
\hline $\mathrm{O} 2^{\mathrm{iii}}-\mathrm{Sr} 1-\mathrm{C}^{\mathrm{ii}}$ & $81.69(4)$ & $\mathrm{C} 3-\mathrm{C} 2-\mathrm{H} 2$ & 120.3 \\
\hline
\end{tabular}




\begin{tabular}{|c|c|c|c|}
\hline $\mathrm{O} 3^{\mathrm{i}}-\mathrm{Sr} 1-\mathrm{C} 7^{\mathrm{iii}}$ & $95.52(4)$ & $\mathrm{C} 1-\mathrm{C} 2-\mathrm{H} 2$ & 120.3 \\
\hline $\mathrm{O} 3-\mathrm{Sr} 1-\mathrm{C} 7^{\mathrm{iii}}$ & $106.98(4)$ & $\mathrm{C} 2-\mathrm{C} 3-\mathrm{C} 4$ & $121.07(17)$ \\
\hline $\mathrm{O} 1 \mathrm{~W}^{\mathrm{i}}-\mathrm{Sr} 1-\mathrm{C} 7^{\mathrm{iii}}$ & $163.73(5)$ & $\mathrm{C} 2-\mathrm{C} 3-\mathrm{H} 3$ & 119.5 \\
\hline $\mathrm{O} 1 \mathrm{~W}-\mathrm{Sr} 1-\mathrm{C}^{\mathrm{iii}}$ & $89.34(5)$ & $\mathrm{C} 4-\mathrm{C} 3-\mathrm{H} 3$ & 119.5 \\
\hline $\mathrm{O} 3^{\mathrm{ii}}-\mathrm{Sr} 1-\mathrm{C} 7^{\mathrm{iii}}$ & $76.00(4)$ & $\mathrm{C} 5-\mathrm{C} 4-\mathrm{C} 3$ & $119.28(18)$ \\
\hline $\mathrm{O} 3^{\mathrm{iii}}-\mathrm{Sr} 1-\mathrm{C} 7^{\mathrm{iii}}$ & $24.45(4)$ & $\mathrm{C} 5-\mathrm{C} 4-\mathrm{H} 4$ & 120.4 \\
\hline $\mathrm{O} 2^{\mathrm{ii}}-\mathrm{Sr} 1-\mathrm{C}^{\mathrm{iii}}$ & $81.69(4)$ & $\mathrm{C} 3-\mathrm{C} 4-\mathrm{H} 4$ & 120.4 \\
\hline $\mathrm{O} 2^{\mathrm{iii}}-\mathrm{Sr} 1-\mathrm{C} 7^{\mathrm{iii}}$ & $24.90(4)$ & $\mathrm{C} 4-\mathrm{C} 5-\mathrm{C} 6$ & $120.99(16)$ \\
\hline $\mathrm{C}^{\mathrm{ii}}-\mathrm{Sr} 1-\mathrm{C}^{\mathrm{iii}}$ & $74.44(6)$ & $\mathrm{C} 4-\mathrm{C} 5-\mathrm{H} 5$ & 119.5 \\
\hline $\mathrm{O} 3^{\mathrm{i}}-\mathrm{Sr} 1-\mathrm{Sr}^{\mathrm{ii}}$ & $161.91(3)$ & $\mathrm{C} 6-\mathrm{C} 5-\mathrm{H} 5$ & 119.5 \\
\hline $\mathrm{O} 3-\mathrm{Sr} 1-\mathrm{Sr} 1^{\mathrm{ii}}$ & $36.87(3)$ & $\mathrm{C} 5-\mathrm{C} 6-\mathrm{C} 1$ & $118.70(16)$ \\
\hline $\mathrm{O} 1 \mathrm{~W}^{\mathrm{i}}-\mathrm{Sr} 1-\mathrm{Sr} 1^{\mathrm{ii}}$ & $78.82(4)$ & $\mathrm{C} 5-\mathrm{C} 6-\mathrm{C} 7$ & $120.60(15)$ \\
\hline $\mathrm{O} 1 \mathrm{~W}-\mathrm{Sr} 1-\mathrm{Sr} 1^{\mathrm{ii}}$ & $124.19(3)$ & $\mathrm{C} 1-\mathrm{C} 6-\mathrm{C} 7$ & $120.69(15)$ \\
\hline $\mathrm{O}^{\mathrm{ii}}-\mathrm{Sr} 1-\mathrm{Sr} 1^{\mathrm{ii}}$ & $35.14(3)$ & $\mathrm{O} 3-\mathrm{C} 7-\mathrm{O} 2$ & $121.30(15)$ \\
\hline $\mathrm{O} 3^{\mathrm{iii}}-\mathrm{Sr} 1-\mathrm{Sr} 1^{\mathrm{ii}}$ & $111.76(3)$ & $\mathrm{O} 3-\mathrm{C} 7-\mathrm{C} 6$ & $119.60(15)$ \\
\hline $\mathrm{O} 2^{\mathrm{ii}}-\mathrm{Sr} 1-\mathrm{Sr} 1^{\mathrm{ii}}$ & $81.81(3)$ & $\mathrm{O} 2-\mathrm{C} 7-\mathrm{C} 6$ & $119.10(15)$ \\
\hline $\mathrm{O} 2^{\mathrm{iii}}-\mathrm{Sr} 1-\mathrm{Sr} 1^{\mathrm{ii}}$ & $74.61(3)$ & $\mathrm{O} 3-\mathrm{C} 7-\mathrm{Ca}^{\mathrm{ii}}$ & $58.13(9)$ \\
\hline $\mathrm{C}^{\mathrm{ii}}-\mathrm{Sr} 1-\mathrm{Sr} 1^{\mathrm{ii}}$ & $58.93(3)$ & $\mathrm{O} 2-\mathrm{C} 7-\mathrm{Ca} 1^{\mathrm{ii}}$ & $64.81(9)$ \\
\hline $\mathrm{C}^{\mathrm{iii}}-\mathrm{Sr} 1-\mathrm{Sr} 1^{\mathrm{ii}}$ & $91.38(3)$ & $\mathrm{C} 6-\mathrm{C} 7-\mathrm{Ca} 1^{\mathrm{ii}}$ & $166.10(12)$ \\
\hline $\mathrm{O} 3^{\mathrm{i}}-\mathrm{Sr} 1-\mathrm{Ca}^{\mathrm{ii}}$ & $161.91(3)$ & $\mathrm{O} 3-\mathrm{C} 7-\mathrm{Sr} 1^{\mathrm{ii}}$ & $58.13(9)$ \\
\hline $\mathrm{O} 3-\mathrm{Sr} 1-\mathrm{Ca} 1^{\mathrm{ii}}$ & $36.87(3)$ & $\mathrm{O} 2-\mathrm{C} 7-\mathrm{Sr}^{\mathrm{ii}}$ & $64.81(9)$ \\
\hline $\mathrm{O} 1 \mathrm{~W}^{\mathrm{i}}-\mathrm{Sr} 1-\mathrm{Ca}^{\mathrm{ii}}$ & $78.82(4)$ & $\mathrm{C} 6-\mathrm{C} 7-\mathrm{Sr}^{\mathrm{ii}}$ & $166.10(12)$ \\
\hline $\mathrm{O} 1 \mathrm{~W}-\mathrm{Sr} 1-\mathrm{Ca} 1^{\mathrm{ii}}$ & $124.19(3)$ & $\mathrm{Ca} 1^{\mathrm{ii}}-\mathrm{C} 7-\mathrm{Sr} 1^{\mathrm{ii}}$ & 0.0 \\
\hline $\mathrm{O} 3^{\mathrm{ii}}-\mathrm{Sr} 1-\mathrm{Ca}^{\mathrm{ii}}$ & $35.14(3)$ & & \\
\hline $\mathrm{O} 1-\mathrm{C} 1-\mathrm{C} 2-\mathrm{C} 3$ & $-177.70(17)$ & $\mathrm{Sr}^{\mathrm{ii}}-\mathrm{O} 3-\mathrm{C} 7-\mathrm{Ca} 1^{\mathrm{ii}}$ & 0.0 \\
\hline $\mathrm{C} 6-\mathrm{C} 1-\mathrm{C} 2-\mathrm{C} 3$ & $2.4(3)$ & $\mathrm{Sr} 1-\mathrm{O} 3-\mathrm{C} 7-\mathrm{Sr} 1^{\mathrm{ii}}$ & $-147.4(2)$ \\
\hline $\mathrm{C} 1-\mathrm{C} 2-\mathrm{C} 3-\mathrm{C} 4$ & $-0.7(3)$ & $\mathrm{Ca} 1^{\mathrm{ii}}-\mathrm{O} 3-\mathrm{C} 7-\mathrm{Sr} 1^{\mathrm{ii}}$ & 0.0 \\
\hline $\mathrm{C} 2-\mathrm{C} 3-\mathrm{C} 4-\mathrm{C} 5$ & $-1.4(3)$ & $\mathrm{Ca} 1^{\mathrm{ii}}-\mathrm{O} 2-\mathrm{C} 7-\mathrm{O} 3$ & $-14.34(17)$ \\
\hline $\mathrm{C} 3-\mathrm{C} 4-\mathrm{C} 5-\mathrm{C} 6$ & $1.9(3)$ & $\mathrm{Sr} 1^{\mathrm{ii}}-\mathrm{O} 2-\mathrm{C} 7-\mathrm{O} 3$ & $-14.34(17)$ \\
\hline $\mathrm{C} 4-\mathrm{C} 5-\mathrm{C} 6-\mathrm{C} 1$ & $-0.2(3)$ & $\mathrm{Ca} 1^{\mathrm{ii}}-\mathrm{O} 2-\mathrm{C} 7-\mathrm{C} 6$ & $164.99(14)$ \\
\hline $\mathrm{C} 4-\mathrm{C} 5-\mathrm{C} 6-\mathrm{C} 7$ & $-179.67(17)$ & $\mathrm{Sr}{ }^{\mathrm{ii}}-\mathrm{O} 2-\mathrm{C} 7-\mathrm{C} 6$ & $164.99(14)$ \\
\hline $\mathrm{O} 1-\mathrm{C} 1-\mathrm{C} 6-\mathrm{C} 5$ & $178.14(16)$ & $\mathrm{Sr} 1^{\mathrm{ii}}-\mathrm{O} 2-\mathrm{C} 7-\mathrm{Ca} 1^{\mathrm{ii}}$ & 0.0 \\
\hline $\mathrm{C} 2-\mathrm{C} 1-\mathrm{C} 6-\mathrm{C} 5$ & $-2.0(3)$ & $\mathrm{Ca} 1^{\mathrm{ii}}-\mathrm{O} 2-\mathrm{C} 7-\mathrm{Sr}^{\mathrm{ii}}$ & 0.0 \\
\hline $\mathrm{O} 1-\mathrm{C} 1-\mathrm{C} 6-\mathrm{C} 7$ & $-2.4(3)$ & $\mathrm{C} 5-\mathrm{C} 6-\mathrm{C} 7-\mathrm{O} 3$ & $19.4(3)$ \\
\hline $\mathrm{C} 2-\mathrm{C} 1-\mathrm{C} 6-\mathrm{C} 7$ & $177.50(16)$ & $\mathrm{C} 1-\mathrm{C} 6-\mathrm{C} 7-\mathrm{O} 3$ & $-160.11(16)$ \\
\hline $\mathrm{Sr} 1-\mathrm{O} 3-\mathrm{C} 7-\mathrm{O} 2$ & $-132.13(19)$ & $\mathrm{C} 5-\mathrm{C} 6-\mathrm{C} 7-\mathrm{O} 2$ & $-159.97(17)$ \\
\hline $\mathrm{Ca} 1^{\mathrm{ii}}-\mathrm{O} 3-\mathrm{C} 7-\mathrm{O} 2$ & $15.30(18)$ & $\mathrm{C} 1-\mathrm{C} 6-\mathrm{C} 7-\mathrm{O} 2$ & $20.6(3)$ \\
\hline $\mathrm{Sr}^{\mathrm{ii}}-\mathrm{O} 3-\mathrm{C} 7-\mathrm{O} 2$ & $15.30(18)$ & $\mathrm{C} 5-\mathrm{C} 6-\mathrm{C} 7-\mathrm{Ca}^{\mathrm{ii}}$ & $-57.2(6)$ \\
\hline $\mathrm{Sr} 1-\mathrm{O} 3-\mathrm{C} 7-\mathrm{C} 6$ & $48.5(3)$ & $\mathrm{C} 1-\mathrm{C} 6-\mathrm{C} 7-\mathrm{Ca}^{\mathrm{ii}}$ & $123.3(4)$ \\
\hline $\mathrm{Ca} 1^{\mathrm{ii}}-\mathrm{O} 3-\mathrm{C} 7-\mathrm{C} 6$ & $-164.02(13)$ & $\mathrm{C} 5-\mathrm{C} 6-\mathrm{C} 7-\mathrm{Sr}^{\mathrm{ii}}$ & $-57.2(6)$ \\
\hline $\mathrm{Sr}^{\mathrm{ii}}-\mathrm{O} 3-\mathrm{C} 7-\mathrm{C} 6$ & $-164.02(13)$ & $\mathrm{C} 1-\mathrm{C} 6-\mathrm{C} 7-\mathrm{Sr}^{\mathrm{ii}}$ & $123.3(4)$ \\
\hline $\mathrm{Sr} 1-\mathrm{O} 3-\mathrm{C} 7-\mathrm{Ca}{ }^{\mathrm{ii}}$ & $-147.4(2)$ & & \\
\hline
\end{tabular}

Symmetry codes: (i) $-x+1, y,-z-1 / 2$; (ii) $-x+1,-y+1,-z$; (iii) $x,-y+1, z-1 / 2$.

Hydrogen-bond geometry $\left(A,{ }^{\circ}\right)$

\begin{tabular}{lllll}
\hline$D-\mathrm{H} \cdots A$ & $D-\mathrm{H}$ & $\mathrm{H} \cdots A$ & $D \cdots A$ & $D-\mathrm{H} \cdots A$ \\
\hline $\mathrm{O} 1-\mathrm{H} 1 O \cdots \mathrm{O} 2$ & $0.89(3)$ & $1.84(3)$ & $2.6031(17)$ & $142(2)$
\end{tabular}




\begin{tabular}{lllll}
$\mathrm{O} 1 W-\mathrm{H} 1 W \cdots \mathrm{O} 1^{\mathrm{iv}}$ & $0.88(3)$ & $1.94(3)$ & $2.8244(18)$ & $173(2)$ \\
$\mathrm{O} 1 W-\mathrm{H} 2 W \cdots \mathrm{O} 2^{\mathrm{v}}$ & $0.85(3)$ & $2.06(3)$ & $2.911(2)$ & $178(2)$ \\
\hline
\end{tabular}

Symmetry codes: (iv) $-x+1 / 2,-y+1 / 2,-z$; (v) $x, y, z-1$.

\section{(CaSr1090)}

\section{Crystal data}

$$
\begin{aligned}
& \mathrm{C}_{14} \mathrm{H}_{14} \mathrm{Ca}_{0.17} \mathrm{O}_{8} \mathrm{Sr}_{0.83} \\
& M_{r}=390.00 \\
& \text { Monoclinic, } C 2 / c \\
& a=16.6693(9) \AA \\
& b=11.4865(7) \AA \\
& c=7.8446(4) \AA \\
& \beta=91.510(5)^{\circ} \\
& V=1501.50(14) \AA^{3} \\
& Z=4
\end{aligned}
$$

\section{Data collection}

Oxford Diffraction Xcalibur E diffractometer

Radiation source: sealed tube $\omega$ scans

\author{
$F(000)=788$ \\ $D_{\mathrm{x}}=1.725 \mathrm{Mg} \mathrm{m}^{-3}$ \\ Mo $K \alpha$ radiation, $\lambda=0.71073 \AA$ \\ Cell parameters from 5187 reflections \\ $\theta=3.3-29.8^{\circ}$ \\ $\mu=3.11 \mathrm{~mm}^{-1}$ \\ $T=150 \mathrm{~K}$ \\ Prism, colourless \\ $0.30 \times 0.15 \times 0.06 \mathrm{~mm}$
}

\section{Refinement}

Refinement on $F^{2}$

Least-squares matrix: full

$R\left[F^{2}>2 \sigma\left(F^{2}\right)\right]=0.027$

$w R\left(F^{2}\right)=0.065$

$S=1.08$

1720 reflections

118 parameters

0 restraints

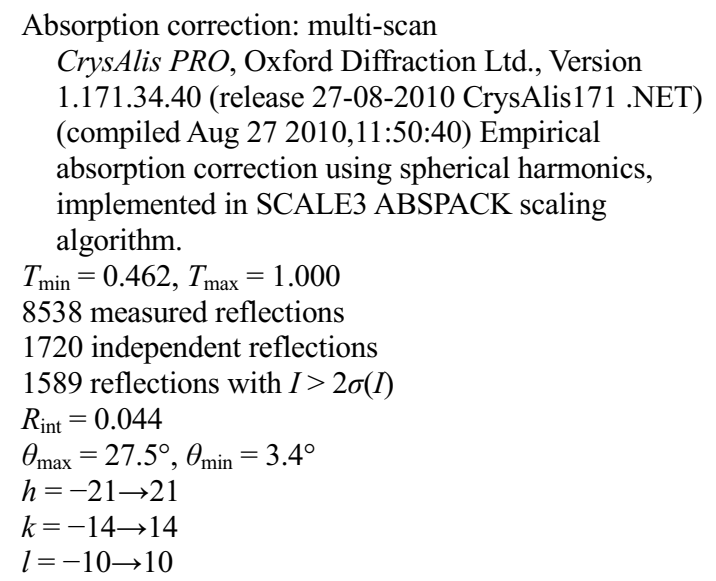

Hydrogen site location: mixed

$\mathrm{H}$ atoms treated by a mixture of independent and constrained refinement

$w=1 /\left[\sigma^{2}\left(F_{\mathrm{o}}^{2}\right)+(0.0342 P)^{2}\right]$

where $P=\left(F_{\mathrm{o}}^{2}+2 F_{\mathrm{c}}{ }^{2}\right) / 3$

$(\Delta / \sigma)_{\max }<0.001$

$\Delta \rho_{\max }=0.70$ e $\AA^{-3}$

$\Delta \rho_{\min }=-0.48$ e $\AA^{-3}$

\section{Special details}

Geometry. All e.s.d.'s (except the e.s.d. in the dihedral angle between two 1.s. planes) are estimated using the full covariance matrix. The cell e.s.d.'s are taken into account individually in the estimation of e.s.d.'s in distances, angles and torsion angles; correlations between e.s.d.'s in cell parameters are only used when they are defined by crystal symmetry. An approximate (isotropic) treatment of cell e.s.d.'s is used for estimating e.s.d.'s involving l.s. planes.

Fractional atomic coordinates and isotropic or equivalent isotropic displacement parameters $\left(\AA^{2}\right)$

\begin{tabular}{llllll}
\hline & $x$ & $y$ & $z$ & $U_{\text {iso }} * / U_{\text {eq }}$ & Occ. $(<1)$ \\
\hline Sr1 & 0.5000 & $0.44477(2)$ & -0.2500 & $0.01276(11)$ & $0.835(5)$ \\
Ca1 & 0.5000 & $0.44477(2)$ & -0.2500 & $0.01276(11)$ & $0.165(5)$ \\
O1 & $0.25676(9)$ & $0.25384(14)$ & $0.26678(18)$ & $0.0233(4)$ &
\end{tabular}




$\begin{array}{lllll}\text { O2 } & 0.37708(9) & 0.39750(13) & 0.26522(17) & 0.0209(4) \\ \text { O3 } & 0.45424(9) & 0.39167(13) & 0.04016(17) & 0.0210(4) \\ \text { O1W } & 0.39510(10) & 0.31466(16) & -0.3837(2) & 0.0290(4) \\ \text { C1 } & 0.30050(12) & 0.18684(18) & 0.1590(2) & 0.0181(4) \\ \text { C2 } & 0.27068(14) & 0.07795(19) & 0.1142(3) & 0.0234(5) \\ \text { H2 } & 0.2203 & 0.0529 & 0.1544 & 0.028^{*} \\ \text { C3 } & 0.31465(15) & 0.0064(2) & 0.0112(3) & 0.0267(5) \\ \text { H3 } & 0.2947 & -0.0688 & -0.0172 & 0.032^{*} \\ \text { C4 } & 0.38764(15) & 0.04259(19) & -0.0520(3) & 0.0279(5) \\ \text { H4 } & 0.4178 & -0.0079 & -0.1217 & 0.033^{*} \\ \text { C5 } & 0.41582(13) & 0.15248(19) & -0.0125(3) & 0.0229(5) \\ \text { H5 } & 0.4651 & 0.1783 & -0.0577 & 0.027^{*} \\ \text { C6 } & 0.37269(12) & 0.22650(18) & 0.0935(2) & 0.0170(4) \\ \text { C7 } & 0.40300(12) & 0.34517(17) & 0.1347(2) & 0.0164(4) \\ \text { H1O } & 0.2833(18) & 0.321(3) & 0.279(4) & 0.050(9)^{*} \\ \text { H1W } & 0.353(2) & 0.289(3) & -0.350(4) & 0.053(10)^{*} \\ \text { H2W } & 0.3914(19) & 0.335(2) & -0.497(4) & 0.054(9)^{*}\end{array}$

Atomic displacement parameters $\left(\AA^{2}\right)$

\begin{tabular}{lllllll}
\hline & $U^{11}$ & $U^{22}$ & $U^{33}$ & $U^{12}$ & $U^{13}$ & $U^{23}$ \\
\hline Sr1 & $0.01047(16)$ & $0.01452(17)$ & $0.01349(14)$ & 0.000 & $0.00403(10)$ & 0.000 \\
Ca1 & $0.01047(16)$ & $0.01452(17)$ & $0.01349(14)$ & 0.000 & $0.00403(10)$ & 0.000 \\
O1 & $0.0175(8)$ & $0.0256(9)$ & $0.0273(8)$ & $-0.0046(7)$ & $0.0095(6)$ & $-0.0026(6)$ \\
O2 & $0.0187(8)$ & $0.0225(8)$ & $0.0219(7)$ & $-0.0029(6)$ & $0.0066(6)$ & $-0.0042(6)$ \\
O3 & $0.0181(8)$ & $0.0221(8)$ & $0.0231(7)$ & $-0.0045(6)$ & $0.0076(6)$ & $0.0001(6)$ \\
O1W & $0.0221(9)$ & $0.0409(11)$ & $0.0243(8)$ & $-0.0111(8)$ & $0.0060(7)$ & $0.0012(7)$ \\
C1 & $0.0156(10)$ & $0.0221(11)$ & $0.0168(9)$ & $-0.0007(8)$ & $0.0019(8)$ & $0.0018(8)$ \\
C2 & $0.0218(11)$ & $0.0256(12)$ & $0.0227(10)$ & $-0.0082(9)$ & $-0.0003(9)$ & $0.0034(8)$ \\
C3 & $0.0368(14)$ & $0.0190(12)$ & $0.0244(11)$ & $-0.0084(10)$ & $-0.0004(10)$ & $-0.0012(9)$ \\
C4 & $0.0338(14)$ & $0.0223(13)$ & $0.0278(11)$ & $0.0014(10)$ & $0.0045(10)$ & $-0.0061(9)$ \\
C5 & $0.0202(11)$ & $0.0243(12)$ & $0.0244(10)$ & $-0.0004(9)$ & $0.0061(9)$ & $-0.0002(8)$ \\
C6 & $0.0159(10)$ & $0.0183(11)$ & $0.0169(9)$ & $-0.0014(8)$ & $0.0008(8)$ & $0.0024(8)$ \\
C7 & $0.0116(9)$ & $0.0194(11)$ & $0.0183(9)$ & $0.0006(8)$ & $0.0010(8)$ & $0.0029(7)$
\end{tabular}

Geometric parameters $\left(\AA,{ }^{o}\right)$

\begin{tabular}{llll}
\hline $\mathrm{Sr} 1-\mathrm{O}^{\mathrm{i}}$ & $2.4953(13)$ & $\mathrm{O} 3-\mathrm{C} 7$ & $1.264(2)$ \\
$\mathrm{Sr} 1-\mathrm{O} 3$ & $2.4953(13)$ & $\mathrm{O} 3-\mathrm{Ca} 1^{\mathrm{ii}}$ & $2.5990(14)$ \\
$\mathrm{Sr} 1-\mathrm{O} 1 W^{\mathrm{i}}$ & $2.5091(17)$ & $\mathrm{O} 3-\mathrm{Sr} 1^{\mathrm{ii}}$ & $2.5990(14)$ \\
$\mathrm{Sr} 1-\mathrm{O} 1 \mathrm{~W}$ & $2.5091(17)$ & $\mathrm{O} 1 \mathrm{~W}-\mathrm{H} 1 \mathrm{~W}$ & $0.81(3)$ \\
$\mathrm{Sr} 1-\mathrm{O} 3^{\mathrm{ii}}$ & $2.5990(14)$ & $\mathrm{O} 1 \mathrm{~W}-\mathrm{H} 2 \mathrm{~W}$ & $0.92(3)$ \\
$\mathrm{Sr} 1-\mathrm{O} 3^{\mathrm{iii}}$ & $2.5990(14)$ & $\mathrm{C} 1-\mathrm{C} 2$ & $1.388(3)$ \\
$\mathrm{Sr} 1-\mathrm{O} 2^{\mathrm{ii}}$ & $2.7400(15)$ & $\mathrm{C} 1-\mathrm{C} 6$ & $1.397(3)$ \\
$\mathrm{Sr} 1-\mathrm{O} 2^{\mathrm{iii}}$ & $2.7400(15)$ & $\mathrm{C} 2-\mathrm{C} 3$ & $1.377(3)$ \\
$\mathrm{Sr} 1-\mathrm{C} 7^{\mathrm{ii}}$ & $3.029(2)$ & $\mathrm{C} 2-\mathrm{H} 2$ & 0.9500 \\
$\mathrm{Sr} 1-\mathrm{C} 7^{\mathrm{iii}}$ & $3.029(2)$ & $\mathrm{C} 3-\mathrm{C} 4$ & $1.389(3)$ \\
$\mathrm{Sr} 1-\mathrm{Sr} 1^{\mathrm{ii}}$ & $4.1224(2)$ & $\mathrm{C} 3-\mathrm{H} 3$ & 0.9500 \\
$\mathrm{Sr} 1-\mathrm{C} 1^{\mathrm{ii}}$ & $4.1224(2)$ & $\mathrm{C} 4-\mathrm{C} 5$ & $1.379(3)$ \\
$\mathrm{Sr} 1-\mathrm{H} 2 \mathrm{~W}$ & $2.90(3)$ & $\mathrm{C} 4-\mathrm{H} 4$ & 0.9500 \\
$\mathrm{O} 1-\mathrm{C} 1$ & $1.368(2)$ & $\mathrm{C} 5-\mathrm{C} 6$ & $1.401(3)$ \\
$\mathrm{O} 1-\mathrm{H} 1 \mathrm{C}$ & $0.90(3)$ & $\mathrm{C} 5-\mathrm{H} 5$ & 0.9500
\end{tabular}




\begin{tabular}{|c|c|}
\hline $\mathrm{O} 2-\mathrm{C} 7$ & $1.273(2)$ \\
\hline $\mathrm{O} 2-\mathrm{Ca}_{1}{ }^{\mathrm{ii}}$ & $2.7400(15)$ \\
\hline $\mathrm{O} 2-\mathrm{Sr} 1^{\mathrm{ii}}$ & $2.7400(15)$ \\
\hline $\mathrm{O}^{3}-\mathrm{Sr} 1-\mathrm{O} 3$ & $151.70(7)$ \\
\hline $\mathrm{O}^{\mathrm{i}}-\mathrm{Sr} 1-\mathrm{O} 1 \mathrm{~W}^{\mathrm{i}}$ & $90.54(5)$ \\
\hline $\mathrm{O} 3-\mathrm{Sr} 1-\mathrm{O} 1 \mathrm{~W}^{\mathrm{i}}$ & $72.51(5)$ \\
\hline $\mathrm{O}^{\mathrm{i}}-\mathrm{Sr} 1-\mathrm{O} 1 \mathrm{~W}$ & $72.51(5)$ \\
\hline $\mathrm{O} 3-\mathrm{Sr} 1-\mathrm{O} 1 \mathrm{~W}$ & $90.54(5)$ \\
\hline $\mathrm{O} 1 \mathrm{~W}^{\mathrm{i}}-\mathrm{Sr} 1-\mathrm{O} 1 \mathrm{~W}$ & $106.88(8)$ \\
\hline $\mathrm{O} 3^{\mathrm{i}}-\mathrm{Sr} 1-\mathrm{O} 3^{\mathrm{ii}}$ & $131.51(5)$ \\
\hline $\mathrm{O} 3-\mathrm{Sr} 1-\mathrm{O} 3^{\mathrm{ii}}$ & $71.98(5)$ \\
\hline $\mathrm{O} 1 \mathrm{~W}^{\mathrm{i}}-\mathrm{Sr} 1-\mathrm{O} 3^{\mathrm{ii}}$ & $88.62(5)$ \\
\hline $\mathrm{O} 1 \mathrm{~W}-\mathrm{Sr} 1-\mathrm{O} 3^{\mathrm{ii}}$ & $152.26(5)$ \\
\hline $\mathrm{O} 3^{\mathrm{i}}-\mathrm{Sr} 1-\mathrm{O} 3^{\mathrm{iii}}$ & $71.98(5)$ \\
\hline $\mathrm{O} 3-\mathrm{Sr} 1-\mathrm{O} 3^{\mathrm{iii}}$ & $131.51(5)$ \\
\hline 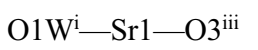 & $152.26(5)$ \\
\hline $\mathrm{O} 1 \mathrm{~W}-\mathrm{Sr} 1-\mathrm{O} 3^{\mathrm{iii}}$ & $88.63(5)$ \\
\hline $\mathrm{O}{ }^{\mathrm{ii}}-\mathrm{Sr} 1-\mathrm{O} 3^{\mathrm{iii}}$ & $87.42(7)$ \\
\hline $\mathrm{O} 3^{\mathrm{i}}-\mathrm{Sr} 1-\mathrm{O} 2^{\mathrm{ii}}$ & $82.82(5)$ \\
\hline $\mathrm{O} 3-\mathrm{Sr} 1-\mathrm{O} 2^{\mathrm{ii}}$ & $116.64(4)$ \\
\hline $\mathrm{O} 1 \mathrm{~W}^{\mathrm{i}}-\mathrm{Sr} 1-\mathrm{O} 2^{\mathrm{ii}}$ & $84.17(5)$ \\
\hline $\mathrm{O} 1 \mathrm{~W}-\mathrm{Sr} 1-\mathrm{O} 2^{\mathrm{ii}}$ & $152.79(5)$ \\
\hline $\mathrm{O} 3^{\mathrm{ii}}-\mathrm{Sr} 1-\mathrm{O} 2^{\mathrm{ii}}$ & $48.88(4)$ \\
\hline $\mathrm{O} 3^{\mathrm{iii}}-\mathrm{Sr} 1-\mathrm{O} 2^{\mathrm{ii}}$ & $72.65(5)$ \\
\hline $\mathrm{O} 3^{\mathrm{i}}-\mathrm{Sr} 1-\mathrm{O} 2^{\mathrm{iii}}$ & $116.64(4)$ \\
\hline $\mathrm{O} 3-\mathrm{Sr} 1-\mathrm{O} 2^{\mathrm{iii}}$ & $82.82(5)$ \\
\hline $\mathrm{O} 1 \mathrm{~W}^{\mathrm{i}}-\mathrm{Sr} 1-\mathrm{O} 2^{\mathrm{iii}}$ & $152.79(5)$ \\
\hline $\mathrm{O} 1 \mathrm{~W}-\mathrm{Sr} 1-\mathrm{O} 2^{\mathrm{iii}}$ & $84.17(5)$ \\
\hline $\mathrm{O} 3^{\mathrm{ii}}-\mathrm{Sr} 1-\mathrm{O} 2^{\mathrm{iii}}$ & $72.65(5)$ \\
\hline $\mathrm{O} 3^{\mathrm{iii}}-\mathrm{Sr} 1-\mathrm{O} 2^{\mathrm{iii}}$ & $48.88(4)$ \\
\hline 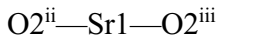 & $97.22(6)$ \\
\hline $\mathrm{O} 3^{\mathrm{i}}-\mathrm{Sr} 1-\mathrm{C} 7^{\mathrm{ii}}$ & $107.07(5)$ \\
\hline $\mathrm{O} 3-\mathrm{Sr} 1-\mathrm{C} 7^{\mathrm{ii}}$ & $95.50(5)$ \\
\hline $\mathrm{O} 1 \mathrm{~W}^{\mathrm{i}}-\mathrm{Sr} 1-\mathrm{C} 7^{\mathrm{ii}}$ & $89.36(5)$ \\
\hline $\mathrm{O} 1 \mathrm{~W}-\mathrm{Sr} 1-\mathrm{C} 7^{\mathrm{ii}}$ & $163.72(6)$ \\
\hline 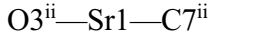 & $24.45(4)$ \\
\hline $\mathrm{O} 3^{\mathrm{iii}}-\mathrm{Sr} 1-\mathrm{C} 7^{\mathrm{ii}}$ & $76.05(5)$ \\
\hline $\mathrm{O} 2^{\mathrm{ii}}-\mathrm{Sr} 1-\mathrm{C} 7^{\mathrm{ii}}$ & $24.85(5)$ \\
\hline $\mathrm{O} 2^{\mathrm{iii}}-\mathrm{Sr} 1-\mathrm{C} 7^{\mathrm{ii}}$ & $81.62(5)$ \\
\hline $\mathrm{O} 3^{\mathrm{i}}-\mathrm{Sr} 1-\mathrm{C} 7^{\mathrm{iii}}$ & $95.50(5)$ \\
\hline $\mathrm{O} 3-\mathrm{Sr} 1-\mathrm{C}^{7 \mathrm{iii}}$ & $107.07(5)$ \\
\hline $\mathrm{O} 1 \mathrm{~W}^{\mathrm{i}}-\mathrm{Sr} 1-\mathrm{C} 7^{\mathrm{iii}}$ & $163.72(6)$ \\
\hline $\mathrm{O} 1 \mathrm{~W}-\mathrm{Sr} 1-\mathrm{C}^{\mathrm{iii}}$ & $89.36(5)$ \\
\hline $\mathrm{O}^{3 \mathrm{ii}}-\mathrm{Sr} 1-\mathrm{C} 7^{\mathrm{iii}}$ & $76.05(5)$ \\
\hline $\mathrm{O} 3^{\mathrm{iii}}-\mathrm{Sr} 1-\mathrm{C} 7^{\mathrm{iii}}$ & $24.45(4)$ \\
\hline $\mathrm{O} 2^{\mathrm{ii}}-\mathrm{Sr} 1-\mathrm{C} 7^{\mathrm{iii}}$ & $81.62(5)$ \\
\hline $\mathrm{O} 2^{\mathrm{iii}}-\mathrm{Sr} 1-\mathrm{C} 7^{\mathrm{iii}}$ & $24.85(5)$ \\
\hline $\mathrm{C} 7^{\mathrm{ii}}-\mathrm{Sr} 1-\mathrm{C} 7^{\mathrm{iii}}$ & $74.42(7)$ \\
\hline $\mathrm{O} 3^{\mathrm{i}}-\mathrm{Sr} 1-\mathrm{Sr} 1^{\mathrm{ii}}$ & $161.96(3)$ \\
\hline $\mathrm{O} 3-\mathrm{Sr} 1-\mathrm{Sr} 1^{\mathrm{ii}}$ & $36.84(3)$ \\
\hline $\mathrm{O} 1 \mathrm{~W}^{\mathrm{i}}-\mathrm{Sr} 1-\mathrm{Sr} 1^{\mathrm{ii}}$ & $78.63(4)$ \\
\hline
\end{tabular}

\begin{tabular}{|c|c|}
\hline $\mathrm{C} 6-\mathrm{C} 7$ & $1.486(3)$ \\
\hline $\mathrm{C} 7-\mathrm{Ca}^{\mathrm{ii}}$ & $3.029(2)$ \\
\hline $\mathrm{C} 7-\mathrm{Sr}^{\mathrm{ii}}$ & $3.029(2)$ \\
\hline $\mathrm{O} 3^{\mathrm{iii}}-\mathrm{Sr} 1-\mathrm{Ca} 1^{\mathrm{ii}}$ & $111.89(3)$ \\
\hline $\mathrm{O} 2^{\mathrm{ii}}-\mathrm{Sr} 1-\mathrm{Ca} 1^{\mathrm{ii}}$ & $81.76(3)$ \\
\hline $\mathrm{O} 2^{\mathrm{iii}}-\mathrm{Sr} 1-\mathrm{Ca} 1^{\mathrm{ii}}$ & $74.71(3)$ \\
\hline $\mathrm{C} 7^{\mathrm{ii}}-\mathrm{Sr} 1-\mathrm{Ca} 1^{\mathrm{ii}}$ & $58.94(4)$ \\
\hline $\mathrm{C}^{\mathrm{iii}}-\mathrm{Sr} 1-\mathrm{Ca} 1^{\mathrm{ii}}$ & 91.47 (4) \\
\hline $\mathrm{Sr} 1^{\mathrm{ii}}-\mathrm{Sr} 1-\mathrm{Ca} 1^{\mathrm{ii}}$ & 0.0 \\
\hline $\mathrm{O}^{\mathrm{i}}-\mathrm{Sr} 1-\mathrm{H} 2 \mathrm{~W}$ & $59.1(6)$ \\
\hline $\mathrm{O} 3-\mathrm{Sr} 1-\mathrm{H} 2 \mathrm{~W}$ & $107.5(6)$ \\
\hline $\mathrm{O} 1 \mathrm{~W}^{\mathrm{i}}-\mathrm{Sr} 1-\mathrm{H} 2 \mathrm{~W}$ & $115.8(6)$ \\
\hline $\mathrm{O} 1 \mathrm{~W}-\mathrm{Sr} 1-\mathrm{H} 2 \mathrm{~W}$ & $17.6(6)$ \\
\hline 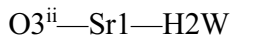 & $154.7(6)$ \\
\hline $\mathrm{O}^{3 \mathrm{ii}}-\mathrm{Sr} 1-\mathrm{H} 2 \mathrm{~W}$ & $74.1(6)$ \\
\hline $\mathrm{O} 2^{\mathrm{ii}}-\mathrm{Sr} 1-\mathrm{H} 2 \mathrm{~W}$ & $135.4(6)$ \\
\hline $\mathrm{O} 2^{\mathrm{iii}}-\mathrm{Sr} 1-\mathrm{H} 2 \mathrm{~W}$ & $82.1(6)$ \\
\hline $\mathrm{C}^{\mathrm{ii}}-\mathrm{Sr} 1-\mathrm{H} 2 \mathrm{~W}$ & $149.8(6)$ \\
\hline $\mathrm{C}^{\mathrm{iii}}-\mathrm{Sr} 1-\mathrm{H} 2 \mathrm{~W}$ & $80.2(6)$ \\
\hline $\mathrm{Sr} 1^{\mathrm{ii}}-\mathrm{Sr} 1-\mathrm{H} 2 \mathrm{~W}$ & $138.8(6)$ \\
\hline $\mathrm{Ca}{ }^{\mathrm{ii}}-\mathrm{Sr} 1-\mathrm{H} 2 \mathrm{~W}$ & $138.8(6)$ \\
\hline $\mathrm{C} 1-\mathrm{O} 1-\mathrm{H} 1 \mathrm{O}$ & $106.4(19)$ \\
\hline $\mathrm{C} 7-\mathrm{O} 2-\mathrm{Ca} 1^{\mathrm{ii}}$ & $90.41(12)$ \\
\hline $\mathrm{C} 7-\mathrm{O} 2-\mathrm{Sr} 1^{\mathrm{ii}}$ & $90.41(12)$ \\
\hline $\mathrm{Ca} 1^{\mathrm{ii}}-\mathrm{O} 2-\mathrm{Sr} 1^{\mathrm{ii}}$ & 0.0 \\
\hline $\mathrm{C} 7-\mathrm{O} 3-\mathrm{Sr} 1$ & $149.97(13)$ \\
\hline $\mathrm{C} 7-\mathrm{O} 3-\mathrm{Ca} 1^{\mathrm{ii}}$ & $97.22(11)$ \\
\hline $\mathrm{Sr} 1-\mathrm{O} 3-\mathrm{Ca} 1^{\mathrm{ii}}$ & 108.0 \\
\hline $\mathrm{C} 7-\mathrm{O} 3-\mathrm{Sr} 1^{\mathrm{ii}}$ & $97.22(11)$ \\
\hline $\mathrm{Sr} 1-\mathrm{O} 3-\mathrm{Sr} 1^{\mathrm{ii}}$ & $108.02(5)$ \\
\hline 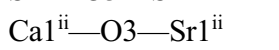 & 0.0 \\
\hline $\mathrm{Sr} 1-\mathrm{O} 1 \mathrm{~W}-\mathrm{H} 1 \mathrm{~W}$ & $133(2)$ \\
\hline $\mathrm{Sr} 1-\mathrm{O} 1 \mathrm{~W}-\mathrm{H} 2 \mathrm{~W}$ & $106.5(19)$ \\
\hline $\mathrm{H} 1 \mathrm{~W}-\mathrm{O} 1 \mathrm{~W}-\mathrm{H} 2 \mathrm{~W}$ & $112(3)$ \\
\hline $\mathrm{O} 1-\mathrm{C} 1-\mathrm{C} 2$ & $118.05(19)$ \\
\hline $\mathrm{O} 1-\mathrm{C} 1-\mathrm{C} 6$ & $121.47(18)$ \\
\hline $\mathrm{C} 2-\mathrm{C} 1-\mathrm{C} 6$ & $120.5(2)$ \\
\hline $\mathrm{C} 3-\mathrm{C} 2-\mathrm{C} 1$ & $119.6(2)$ \\
\hline $\mathrm{C} 3-\mathrm{C} 2-\mathrm{H} 2$ & 120.2 \\
\hline $\mathrm{C} 1-\mathrm{C} 2-\mathrm{H} 2$ & 120.2 \\
\hline $\mathrm{C} 2-\mathrm{C} 3-\mathrm{C} 4$ & $121.1(2)$ \\
\hline $\mathrm{C} 2-\mathrm{C} 3-\mathrm{H} 3$ & 119.5 \\
\hline $\mathrm{C} 4-\mathrm{C} 3-\mathrm{H} 3$ & 119.5 \\
\hline $\mathrm{C} 5-\mathrm{C} 4-\mathrm{C} 3$ & $119.3(2)$ \\
\hline $\mathrm{C} 5-\mathrm{C} 4-\mathrm{H} 4$ & 120.3 \\
\hline $\mathrm{C} 3-\mathrm{C} 4-\mathrm{H} 4$ & 120.3 \\
\hline $\mathrm{C} 4-\mathrm{C} 5-\mathrm{C} 6$ & $120.8(2)$ \\
\hline $\mathrm{C} 4-\mathrm{C} 5-\mathrm{H} 5$ & 119.6 \\
\hline $\mathrm{C} 6-\mathrm{C} 5-\mathrm{H} 5$ & 119.6 \\
\hline $\mathrm{C} 1-\mathrm{C} 6-\mathrm{C} 5$ & $118.74(19)$ \\
\hline $\mathrm{C} 1-\mathrm{C} 6-\mathrm{C} 7$ & $120.69(18)$ \\
\hline
\end{tabular}




\begin{tabular}{|c|c|c|c|}
\hline $\mathrm{O} 1 \mathrm{~W}-\mathrm{Sr} 1-\mathrm{Sr} 1^{\mathrm{ii}}$ & $124.31(4)$ & $\mathrm{C} 5-\mathrm{C} 6-\mathrm{C} 7$ & $120.58(18)$ \\
\hline $\mathrm{O}^{\mathrm{ii}}-\mathrm{Sr} 1-\mathrm{Sr} 1^{\mathrm{ii}}$ & $35.14(3)$ & $\mathrm{O} 3-\mathrm{C} 7-\mathrm{O} 2$ & $121.42(19)$ \\
\hline $\mathrm{O} 3{ }^{\mathrm{iii}}-\mathrm{Sr} 1-\mathrm{Sr}^{\mathrm{ii}}$ & $111.89(3)$ & $\mathrm{O} 3-\mathrm{C} 7-\mathrm{C} 6$ & $119.43(17)$ \\
\hline $\mathrm{O} 2^{\mathrm{ii}}-\mathrm{Sr} 1-\mathrm{Sr} 1^{\mathrm{ii}}$ & $81.76(3)$ & $\mathrm{O} 2-\mathrm{C} 7-\mathrm{C} 6$ & $119.16(17)$ \\
\hline $\mathrm{O} 2^{\mathrm{iii}}-\mathrm{Sr} 1-\mathrm{Sr} 1^{\mathrm{ii}}$ & $74.71(3)$ & $\mathrm{O} 3-\mathrm{C} 7-\mathrm{Ca}^{\mathrm{ii}}$ & $58.33(10)$ \\
\hline $\mathrm{C}^{\mathrm{ii}}-\mathrm{Sr} 1-\mathrm{Sr} 1^{\mathrm{ii}}$ & $58.94(4)$ & $\mathrm{O} 2-\mathrm{C} 7-\mathrm{Ca}^{\mathrm{ii}}$ & $64.75(11)$ \\
\hline $\mathrm{C} 7^{\mathrm{iii}}-\mathrm{Sr} 1-\mathrm{Sr} 1^{\mathrm{ii}}$ & $91.47(4)$ & $\mathrm{C} 6-\mathrm{C} 7-\mathrm{Ca}^{\mathrm{ii}}$ & $166.17(14)$ \\
\hline $\mathrm{O} 3^{\mathrm{i}}-\mathrm{Sr} 1-\mathrm{Ca} 1^{\mathrm{ii}}$ & $161.96(3)$ & $\mathrm{O} 3-\mathrm{C} 7-\mathrm{Sr}^{\mathrm{ii}}$ & $58.33(10)$ \\
\hline $\mathrm{O} 3-\mathrm{Sr} 1-\mathrm{Ca} 1^{\mathrm{ii}}$ & $36.84(3)$ & $\mathrm{O} 2-\mathrm{C} 7-\mathrm{Sr}^{\mathrm{ii}}$ & $64.75(11)$ \\
\hline $\mathrm{O} 1 \mathrm{~W}^{\mathrm{i}}-\mathrm{Sr} 1-\mathrm{Ca}^{\mathrm{ii}}$ & $78.63(4)$ & $\mathrm{C} 6-\mathrm{C} 7-\mathrm{Sr}^{\mathrm{ii}}$ & $166.17(14)$ \\
\hline $\mathrm{O} 1 \mathrm{~W}-\mathrm{Sr} 1-\mathrm{Ca}^{\mathrm{ii}}$ & $124.31(4)$ & $\mathrm{Ca} 1^{\mathrm{ii}}-\mathrm{C} 7-\mathrm{Sr} 1^{\mathrm{ii}}$ & 0.0 \\
\hline $\mathrm{O} 3^{\mathrm{ii}}-\mathrm{Sr} 1-\mathrm{Ca} 1^{\mathrm{ii}}$ & $35.14(3)$ & & \\
\hline $\mathrm{O} 1-\mathrm{C} 1-\mathrm{C} 2-\mathrm{C} 3$ & $-177.54(19)$ & $\mathrm{Sr}^{\mathrm{ii}}-\mathrm{O} 3-\mathrm{C} 7-\mathrm{Ca}^{\mathrm{ii}}$ & $0.000(1)$ \\
\hline $\mathrm{C} 6-\mathrm{C} 1-\mathrm{C} 2-\mathrm{C} 3$ & $3.2(3)$ & $\mathrm{Sr} 1-\mathrm{O} 3-\mathrm{C} 7-\mathrm{Sr}{ }^{\mathrm{ii}}$ & $-147.4(3)$ \\
\hline $\mathrm{C} 1-\mathrm{C} 2-\mathrm{C} 3-\mathrm{C} 4$ & $-1.5(3)$ & $\mathrm{Ca} 1^{\mathrm{ii}}-\mathrm{O} 3-\mathrm{C} 7-\mathrm{Sr} 1^{\mathrm{ii}}$ & $0.000(1)$ \\
\hline $\mathrm{C} 2-\mathrm{C} 3-\mathrm{C} 4-\mathrm{C} 5$ & $-0.9(3)$ & $\mathrm{Ca} 1^{\mathrm{ii}}-\mathrm{O} 2-\mathrm{C} 7-\mathrm{O} 3$ & $-14.47(19)$ \\
\hline $\mathrm{C} 3-\mathrm{C} 4-\mathrm{C} 5-\mathrm{C} 6$ & $1.5(3)$ & $\mathrm{Sr} 1^{\mathrm{ii}}-\mathrm{O} 2-\mathrm{C} 7-\mathrm{O} 3$ & $-14.47(19)$ \\
\hline $\mathrm{O} 1-\mathrm{C} 1-\mathrm{C} 6-\mathrm{C} 5$ & $178.24(18)$ & $\mathrm{Ca} 1^{\mathrm{ii}}-\mathrm{O} 2-\mathrm{C} 7-\mathrm{C} 6$ & $165.07(16)$ \\
\hline $\mathrm{C} 2-\mathrm{C} 1-\mathrm{C} 6-\mathrm{C} 5$ & $-2.5(3)$ & $\mathrm{Sr} 1^{\mathrm{ii}}-\mathrm{O} 2-\mathrm{C} 7-\mathrm{C} 6$ & $165.07(16)$ \\
\hline $\mathrm{O} 1-\mathrm{C} 1-\mathrm{C} 6-\mathrm{C} 7$ & $-2.1(3)$ & $\mathrm{Sr} 1^{\mathrm{ii}}-\mathrm{O} 2-\mathrm{C} 7-\mathrm{Ca} 1^{\mathrm{ii}}$ & 0.0 \\
\hline $\mathrm{C} 2-\mathrm{C} 1-\mathrm{C} 6-\mathrm{C} 7$ & $177.16(18)$ & $\mathrm{Ca} 1^{\mathrm{ii}}-\mathrm{O} 2-\mathrm{C} 7-\mathrm{Sr} 1^{\mathrm{ii}}$ & 0.0 \\
\hline $\mathrm{C} 4-\mathrm{C} 5-\mathrm{C} 6-\mathrm{C} 1$ & $0.1(3)$ & $\mathrm{C} 1-\mathrm{C} 6-\mathrm{C} 7-\mathrm{O} 3$ & $-160.15(18)$ \\
\hline $\mathrm{C} 4-\mathrm{C} 5-\mathrm{C} 6-\mathrm{C} 7$ & $-179.5(2)$ & $\mathrm{C} 5-\mathrm{C} 6-\mathrm{C} 7-\mathrm{O} 3$ & $19.5(3)$ \\
\hline $\mathrm{Sr} 1-\mathrm{O} 3-\mathrm{C} 7-\mathrm{O} 2$ & $-132.0(2)$ & $\mathrm{C} 1-\mathrm{C} 6-\mathrm{C} 7-\mathrm{O} 2$ & $20.3(3)$ \\
\hline $\mathrm{Ca} 1^{\mathrm{ii}}-\mathrm{O} 3-\mathrm{C} 7-\mathrm{O} 2$ & $15.4(2)$ & $\mathrm{C} 5-\mathrm{C} 6-\mathrm{C} 7-\mathrm{O} 2$ & $-160.04(19)$ \\
\hline $\mathrm{Sr}^{\mathrm{ii}}-\mathrm{O} 3-\mathrm{C} 7-\mathrm{O} 2$ & $15.4(2)$ & $\mathrm{C} 1-\mathrm{C} 6-\mathrm{C} 7-\mathrm{Ca} 1^{\mathrm{ii}}$ & $123.2(5)$ \\
\hline $\mathrm{Sr} 1-\mathrm{O} 3-\mathrm{C} 7-\mathrm{C} 6$ & $48.5(4)$ & $\mathrm{C} 5-\mathrm{C} 6-\mathrm{C} 7-\mathrm{Ca}^{\mathrm{ii}}$ & $-57.2(6)$ \\
\hline $\mathrm{Ca} 1^{\mathrm{ii}}-\mathrm{O} 3-\mathrm{C} 7-\mathrm{C} 6$ & $-164.14(15)$ & $\mathrm{C} 1-\mathrm{C} 6-\mathrm{C} 7-\mathrm{Sr}^{\mathrm{ii}}$ & $123.2(5)$ \\
\hline $\mathrm{Sr} 1^{\mathrm{ii}}-\mathrm{O} 3-\mathrm{C} 7-\mathrm{C} 6$ & $-164.14(15)$ & $\mathrm{C} 5-\mathrm{C} 6-\mathrm{C} 7-\mathrm{Sr}^{\mathrm{ii}}$ & $-57.2(6)$ \\
\hline $\mathrm{Sr} 1-\mathrm{O} 3-\mathrm{C} 7-\mathrm{Ca}^{\mathrm{ii}}$ & $-147.4(3)$ & & \\
\hline
\end{tabular}

Symmetry codes: (i) $-x+1, y,-z-1 / 2$; (ii) $-x+1,-y+1,-z$; (iii) $x,-y+1, z-1 / 2$.

Hydrogen-bond geometry $\left(A,{ }^{\circ}\right)$

\begin{tabular}{lllll}
\hline$D-\mathrm{H} \cdots A$ & $D-\mathrm{H}$ & $\mathrm{H} \cdots A$ & $D \cdots A$ & $D-\mathrm{H} \cdots A$ \\
\hline $\mathrm{O} 1-\mathrm{H} 1 O \cdots \mathrm{O} 2$ & $0.90(3)$ & $1.80(3)$ & $2.597(2)$ & $147(3)$ \\
$\mathrm{O} 1 W-\mathrm{H} 1 W \cdots \mathrm{O} 1^{\text {iv }}$ & $0.81(3)$ & $2.03(3)$ & $2.827(2)$ & $173(3)$ \\
$\mathrm{O} 1 W-\mathrm{H} 2 W \cdots \mathrm{O} 2^{\mathrm{v}}$ & $0.92(3)$ & $2.01(3)$ & $2.921(2)$ & $173(3)$ \\
\hline
\end{tabular}

Symmetry codes: (iv) $-x+1 / 2,-y+1 / 2,-z$; (v) $x, y, z-1$.

\section{(Sr100)}

\section{Crystal data}

$\mathrm{C}_{14} \mathrm{H}_{14} \mathrm{O}_{8} \mathrm{Sr}$

$M_{r}=397.87$

Monoclinic, $C 2 / c$

$a=16.7182(6) \AA$

$b=11.4644$ (4) $\AA$

$c=7.8627$ (3) $\AA$

$\beta=91.660(3)^{\circ}$
$V=1506.37$ (9) $\AA^{3}$

$Z=4$

$F(000)=800$

$D_{\mathrm{x}}=1.754 \mathrm{Mg} \mathrm{m}^{-3}$

$\mathrm{Cu} K \alpha$ radiation, $\lambda=1.5418 \AA$

Cell parameters from 1700 reflections

$\theta=5.3-65.9^{\circ}$ 
$\mu=5.36 \mathrm{~mm}^{-1}$

$T=150 \mathrm{~K}$

\section{Data collection}

Oxford Diffraction Gemini S diffractometer

Radiation source: sealed tube $\omega$ scans
Prism, colourless

$0.6 \times 0.5 \times 0.2 \mathrm{~mm}$

\section{Refinement}

Refinement on $F^{2}$

Least-squares matrix: full

$R\left[F^{2}>2 \sigma\left(F^{2}\right)\right]=0.027$

$w R\left(F^{2}\right)=0.073$

$S=1.17$

1272 reflections

118 parameters

3 restraints

Hydrogen site location: mixed

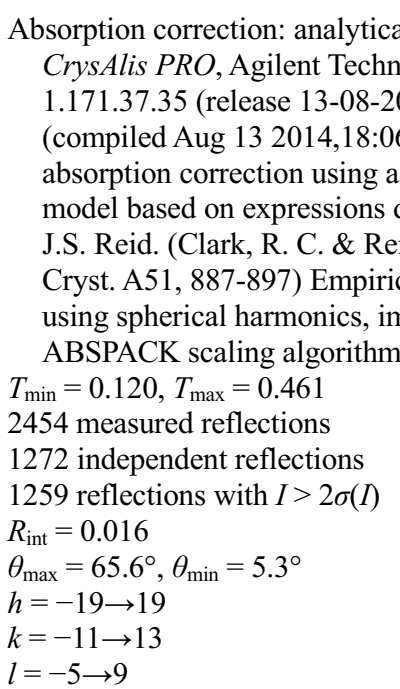

$\mathrm{H}$ atoms treated by a mixture of independent and constrained refinement

$w=1 /\left[\sigma^{2}\left(F_{\mathrm{o}}{ }^{2}\right)+(0.0464 P)^{2}+2.8711 P\right]$

where $P=\left(F_{\mathrm{o}}{ }^{2}+2 F_{\mathrm{c}}{ }^{2}\right) / 3$

$(\Delta / \sigma)_{\max }<0.001$

$\Delta \rho_{\max }=0.68$ e $\AA^{-3}$

$\Delta \rho_{\min }=-0.49$ e $\AA^{-3}$

Extinction correction: SHELXL2014/7 (Sheldrick $2015, \mathrm{Fc}^{*}=\mathrm{kFc}\left[1+0.001 \mathrm{xFc}^{2} \lambda^{3} / \sin (2 \theta)\right]^{-1 / 4}$

Extinction coefficient: 0.0030 (2)

\section{Special details}

Geometry. All e.s.d.'s (except the e.s.d. in the dihedral angle between two l.s. planes) are estimated using the full covariance matrix. The cell e.s.d.'s are taken into account individually in the estimation of e.s.d.'s in distances, angles and torsion angles; correlations between e.s.d.'s in cell parameters are only used when they are defined by crystal symmetry. An approximate (isotropic) treatment of cell e.s.d.'s is used for estimating e.s.d.'s involving l.s. planes.

Fractional atomic coordinates and isotropic or equivalent isotropic displacement parameters $\left(\hat{A}^{2}\right)$

\begin{tabular}{lllll}
\hline & $x$ & $y$ & $z$ & $U_{\text {iso }} * / U_{\text {eq }}$ \\
\hline Sr1 & 0.5000 & $0.44431(2)$ & -0.2500 & $0.00935(19)$ \\
O1 & $0.25630(11)$ & $0.25371(16)$ & $0.2660(2)$ & $0.0170(4)$ \\
O2 & $0.37709(10)$ & $0.39712(15)$ & $0.2644(2)$ & $0.0140(4)$ \\
O3 & $0.45408(10)$ & $0.39012(16)$ & $0.0409(2)$ & $0.0143(4)$ \\
O1W & $0.39465(12)$ & $0.31301(18)$ & $-0.3853(3)$ & $0.0200(4)$ \\
C1 & $0.29980(15)$ & $0.1867(2)$ & $0.1585(3)$ & $0.0130(5)$ \\
C2 & $0.26953(17)$ & $0.0777(2)$ & $0.1141(4)$ & $0.0173(6)$ \\
H2 & 0.2193 & 0.0529 & 0.1544 & $0.021^{*}$ \\
C3 & $0.31329(18)$ & $0.0055(2)$ & $0.0107(4)$ & $0.0198(6)$ \\
H3 & 0.2932 & -0.0696 & -0.0182 & $0.024^{*}$ \\
C4 & $0.3864(2)$ & $0.0415(2)$ & $-0.0518(4)$ & $0.0212(6)$ \\
H4 & 0.4165 & -0.0092 & -0.1211 & $0.025^{*}$
\end{tabular}


supporting information

\begin{tabular}{lllll} 
C5 & $0.41481(17)$ & $0.1514(2)$ & $-0.0121(3)$ & $0.0166(6)$ \\
H5 & 0.4639 & 0.1770 & -0.0569 & $0.020^{*}$ \\
C6 & $0.37212(15)$ & $0.2254(2)$ & $0.0934(3)$ & $0.0124(5)$ \\
C7 & $0.40301(15)$ & $0.3448(2)$ & $0.1348(3)$ & $0.0114(5)$ \\
H1O & $0.279(2)$ & $0.317(2)$ & $0.279(5)$ & $0.031(10)^{*}$ \\
H1W & $0.3533(15)$ & $0.289(3)$ & $-0.344(4)$ & $0.029(10)^{*}$ \\
H2W & $0.391(2)$ & $0.336(3)$ & $-0.487(3)$ & $0.030(10)^{*}$ \\
\hline
\end{tabular}

Atomic displacement parameters $\left(\AA^{2}\right)$

\begin{tabular}{lllllll}
\hline & $U^{11}$ & $U^{22}$ & $U^{33}$ & $U^{12}$ & $U^{13}$ & $U^{23}$ \\
\hline Sr1 & $0.0106(2)$ & $0.0095(2)$ & $0.0081(2)$ & 0.000 & $0.00290(13)$ & 0.000 \\
O1 & $0.0162(9)$ & $0.0165(10)$ & $0.0185(10)$ & $-0.0050(8)$ & $0.0071(7)$ & $-0.0010(8)$ \\
O2 & $0.0150(9)$ & $0.0141(9)$ & $0.0132(9)$ & $-0.0021(7)$ & $0.0054(7)$ & $-0.0024(7)$ \\
O3 & $0.0149(9)$ & $0.0148(9)$ & $0.0134(9)$ & $-0.0040(7)$ & $0.0049(7)$ & $-0.0007(7)$ \\
O1W & $0.0181(10)$ & $0.0281(11)$ & $0.0140(10)$ & $-0.0080(8)$ & $0.0040(8)$ & $0.0006(8)$ \\
C1 & $0.0150(12)$ & $0.0150(12)$ & $0.0089(12)$ & $-0.0001(10)$ & $-0.0001(10)$ & $0.0025(10)$ \\
C2 & $0.0193(14)$ & $0.0179(12)$ & $0.0145(13)$ & $-0.0069(11)$ & $-0.0004(11)$ & $0.0040(11)$ \\
C3 & $0.0317(16)$ & $0.0112(13)$ & $0.0164(13)$ & $-0.0058(11)$ & $-0.0022(12)$ & $-0.0009(10)$ \\
C4 & $0.0302(17)$ & $0.0157(13)$ & $0.0179(15)$ & $0.0015(11)$ & $0.0036(13)$ & $-0.0038(11)$ \\
C5 & $0.0185(13)$ & $0.0172(13)$ & $0.0141(13)$ & $0.0002(11)$ & $0.0038(10)$ & $0.0003(10)$ \\
C6 & $0.0141(12)$ & $0.0126(12)$ & $0.0106(12)$ & $0.0002(10)$ & $-0.0006(10)$ & $0.0021(10)$ \\
C7 & $0.0103(12)$ & $0.0128(12)$ & $0.0111(12)$ & $-0.0001(9)$ & $-0.0010(10)$ & $0.0028(10)$ \\
\hline
\end{tabular}

Geometric parameters ( $\left.A,{ }^{\circ}\right)$

\begin{tabular}{|c|c|c|c|}
\hline $\mathrm{Sr} 1-\mathrm{O} 3$ & $2.5116(17)$ & $\mathrm{O} 3-\mathrm{C} 7$ & $1.257(3)$ \\
\hline $\mathrm{Sr} 1-\mathrm{O}^{\mathrm{i}}$ & $2.5116(17)$ & $\mathrm{O} 3-\mathrm{Sr} 1^{\mathrm{ii}}$ & $2.6121(17)$ \\
\hline $\mathrm{Sr} 1-\mathrm{O} 1 \mathrm{~W}$ & $2.528(2)$ & $\mathrm{O} 1 \mathrm{~W}-\mathrm{H} 1 \mathrm{~W}$ & $0.819(19)$ \\
\hline $\mathrm{Sr} 1-\mathrm{O} 1 \mathrm{~W}^{\mathrm{i}}$ & $2.528(2)$ & $\mathrm{O} 1 \mathrm{~W}-\mathrm{H} 2 \mathrm{~W}$ & $0.841(19)$ \\
\hline $\mathrm{Sr} 1-\mathrm{O} 3^{\mathrm{ii}}$ & $2.6120(17)$ & $\mathrm{C} 1-\mathrm{C} 2$ & $1.389(4)$ \\
\hline $\mathrm{Sr} 1-\mathrm{O}^{\mathrm{iii}}$ & $2.6120(17)$ & $\mathrm{C} 1-\mathrm{C} 6$ & $1.399(4)$ \\
\hline $\mathrm{Sr} 1-\mathrm{O} 2^{\mathrm{ii}}$ & $2.7484(17)$ & $\mathrm{C} 2-\mathrm{C} 3$ & $1.384(4)$ \\
\hline $\mathrm{Sr} 1-\mathrm{O} 2^{\mathrm{iii}}$ & $2.7484(17)$ & $\mathrm{C} 2-\mathrm{H} 2$ & 0.9500 \\
\hline $\mathrm{Sr} 1-\mathrm{C} 7^{\mathrm{ii}}$ & $3.035(3)$ & $\mathrm{C} 3-\mathrm{C} 4$ & $1.393(4)$ \\
\hline $\operatorname{Sr} 1-C 7^{7 i i}$ & $3.035(3)$ & $\mathrm{C} 3-\mathrm{H} 3$ & 0.9500 \\
\hline $\mathrm{Sr} 1-\mathrm{Sr} 1^{\mathrm{ii}}$ & $4.1335(2)$ & $\mathrm{C} 4-\mathrm{C} 5$ & $1.379(4)$ \\
\hline $\mathrm{Sr} 1-\mathrm{Sr} 1^{\mathrm{iv}}$ & $4.1335(2)$ & $\mathrm{C} 4-\mathrm{H} 4$ & 0.9500 \\
\hline $\mathrm{Sr} 1-\mathrm{H} 2 \mathrm{~W}$ & $2.86(3)$ & $\mathrm{C} 5-\mathrm{C} 6$ & $1.397(4)$ \\
\hline $\mathrm{O} 1-\mathrm{C} 1$ & $1.367(3)$ & $\mathrm{C} 5-\mathrm{H} 5$ & 0.9500 \\
\hline $\mathrm{O} 1-\mathrm{H} 1 \mathrm{O}$ & $0.825(19)$ & $\mathrm{C} 6-\mathrm{C} 7$ & $1.496(4)$ \\
\hline $\mathrm{O} 2-\mathrm{C} 7$ & $1.270(3)$ & $\mathrm{C} 7-\mathrm{Sr}^{\mathrm{ii}}$ & $3.035(3)$ \\
\hline $\mathrm{O} 2-\mathrm{Sr} 1^{\mathrm{ii}}$ & $2.7484(17)$ & & \\
\hline $\mathrm{O} 3-\mathrm{Sr} 1-\mathrm{O}^{\mathrm{i}}$ & $151.36(8)$ & $\mathrm{O} 3-\mathrm{Sr} 1-\mathrm{Sr} 1^{\mathrm{iv}}$ & $161.98(4)$ \\
\hline $\mathrm{O} 3-\mathrm{Sr} 1-\mathrm{O} 1 \mathrm{~W}$ & $90.48(6)$ & $\mathrm{O} 3^{\mathrm{i}}-\mathrm{Sr} 1-\mathrm{Sr} 1^{\mathrm{iv}}$ & $37.05(4)$ \\
\hline $\mathrm{O} 33^{\mathrm{i}}-\mathrm{Sr} 1-\mathrm{O} 1 \mathrm{~W}$ & $72.37(6)$ & $\mathrm{O} 1 \mathrm{~W}-\mathrm{Sr} 1-\mathrm{Sr}^{\mathrm{iv}}$ & $78.63(5)$ \\
\hline $\mathrm{O} 3-\mathrm{Sr} 1-\mathrm{O}_{1} \mathrm{~W}^{\mathrm{i}}$ & $72.37(6)$ & $\mathrm{O} 1 \mathrm{~W}^{\mathrm{i}}-\mathrm{Sr} 1-\mathrm{Sr} 1^{\mathrm{iv}}$ & $124.40(4)$ \\
\hline $\mathrm{O} 3^{\mathrm{i}}-\mathrm{Sr} 1-\mathrm{O} 1 \mathrm{~W}^{\mathrm{i}}$ & $90.48(6)$ & $\mathrm{O}^{\mathrm{ii}}-\mathrm{Sr} 1-\mathrm{Sr} 1^{\mathrm{iv}}$ & $111.47(4)$ \\
\hline $\mathrm{O} 1 \mathrm{~W}-\mathrm{Sr} 1-\mathrm{O} 1 \mathrm{~W}^{\mathrm{i}}$ & $106.92(10)$ & $\mathrm{O}^{\mathrm{iii}}-\mathrm{Sr} 1-\mathrm{Sr} 1^{\mathrm{iv}}$ & $35.40(4)$ \\
\hline $\mathrm{O} 3-\mathrm{Sr} 1-\mathrm{O} 3^{\mathrm{ii}}$ & $72.46(6)$ & $\mathrm{O} 2^{\mathrm{ii}}-\mathrm{Sr} 1-\mathrm{Sr} 1^{\mathrm{iv}}$ & $74.68(4)$ \\
\hline $\mathrm{O} 3^{\mathrm{i}}-\mathrm{Sr} 1-\mathrm{O} 3^{\mathrm{ii}}$ & $131.37(6)$ & $\mathrm{O} 2^{\mathrm{iii}}-\mathrm{Sr} 1-\mathrm{Sr} 1^{\mathrm{iv}}$ & $81.70(4)$ \\
\hline
\end{tabular}




\begin{tabular}{|c|c|c|c|}
\hline $\mathrm{O} 1 \mathrm{~W}-\mathrm{Sr} 1-\mathrm{O} 3^{\mathrm{ii}}$ & $152.38(6)$ & $\mathrm{C} 7^{\mathrm{ii}}-\mathrm{Sr} 1-\mathrm{Sr} 1^{\mathrm{iv}}$ & $91.32(5)$ \\
\hline $\mathrm{O} 1 \mathrm{~W}^{\mathrm{i}}-\mathrm{Sr} 1-\mathrm{O}^{\mathrm{ii}}$ & $88.82(6)$ & 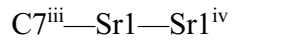 & $58.98(5)$ \\
\hline $\mathrm{O} 3-\mathrm{Sr} 1-\mathrm{O} 3^{\mathrm{iii}}$ & $131.37(6)$ & $\mathrm{Sr} 1^{\mathrm{ii}}-\mathrm{Sr} 1-\mathrm{Sr} 1^{\mathrm{iv}}$ & $144.010(14)$ \\
\hline $\mathrm{O} 3^{\mathrm{i}}-\mathrm{Sr} 1-\mathrm{O} 3^{\mathrm{iii}}$ & $72.46(6)$ & $\mathrm{O} 3-\mathrm{Sr} 1-\mathrm{H} 2 \mathrm{~W}$ & $106.2(5)$ \\
\hline $\mathrm{O} 1 \mathrm{~W}-\mathrm{Sr} 1-\mathrm{O} 3^{\mathrm{iii}}$ & $88.82(6)$ & $\mathrm{O} 3{ }^{\mathrm{i}}-\mathrm{Sr} 1-\mathrm{H} 2 \mathrm{~W}$ & $60.4(6)$ \\
\hline $\mathrm{O} 1 \mathrm{~W}^{\mathrm{i}}-\mathrm{Sr} 1-\mathrm{O} 3^{\mathrm{iii}}$ & $152.38(6)$ & $\mathrm{O} 1 \mathrm{~W}-\mathrm{Sr} 1-\mathrm{H} 2 \mathrm{~W}$ & $16.6(5)$ \\
\hline $\mathrm{O} 3^{\mathrm{ii}}-\mathrm{Sr} 1-\mathrm{O} 3^{\mathrm{iii}}$ & $86.78(8)$ & $\mathrm{O} 1 \mathrm{~W}^{\mathrm{i}}-\mathrm{Sr} 1-\mathrm{H} 2 \mathrm{~W}$ & $116.2(7)$ \\
\hline $\mathrm{O} 3-\mathrm{Sr} 1-\mathrm{O} 2^{\mathrm{ii}}$ & $116.73(5)$ & $\mathrm{O} 3{ }^{\mathrm{ii}}-\mathrm{Sr} 1-\mathrm{H} 2 \mathrm{~W}$ & $153.8(7)$ \\
\hline $\mathrm{O} 3^{\mathrm{i}}-\mathrm{Sr} 1-\mathrm{O} 2^{\mathrm{ii}}$ & $82.96(5)$ & $\mathrm{O} 3^{\mathrm{iii}}-\mathrm{Sr} 1-\mathrm{H} 2 \mathrm{~W}$ & $74.6(6)$ \\
\hline $\mathrm{O} 1 \mathrm{~W}-\mathrm{Sr} 1-\mathrm{O} 2^{\mathrm{ii}}$ & $152.75(6)$ & $\mathrm{O} 2^{\mathrm{ii}}-\mathrm{Sr} 1-\mathrm{H} 2 \mathrm{~W}$ & $136.6(5)$ \\
\hline $\mathrm{O} 1 \mathrm{~W}^{\mathrm{i}}-\mathrm{Sr} 1-\mathrm{O} 2^{\mathrm{ii}}$ & $84.18(6)$ & $\mathrm{O} 2^{\mathrm{iii}}-\mathrm{Sr} 1-\mathrm{H} 2 \mathrm{~W}$ & $81.3(7)$ \\
\hline $\mathrm{O} 3^{\mathrm{ii}}-\mathrm{Sr} 1-\mathrm{O} 2^{\mathrm{ii}}$ & $48.61(5)$ & $\mathrm{C}^{\mathrm{ii}}-\mathrm{Sr} 1-\mathrm{H} 2 \mathrm{~W}$ & $150.2(6)$ \\
\hline $\mathrm{O} 3^{\mathrm{iii}}-\mathrm{Sr} 1-\mathrm{O} 2^{\mathrm{ii}}$ & $72.53(5)$ & $\mathrm{C}^{7 \mathrm{ii}}-\mathrm{Sr} 1-\mathrm{H} 2 \mathrm{~W}$ & $79.9(7)$ \\
\hline $\mathrm{O} 3-\mathrm{Sr} 1-\mathrm{O} 2^{\mathrm{iii}}$ & $82.96(5)$ & $\mathrm{Sr} 1{ }^{\mathrm{ii}}-\mathrm{Sr} 1-\mathrm{H} 2 \mathrm{~W}$ & $137.4(6)$ \\
\hline $\mathrm{O} 3^{\mathrm{i}}-\mathrm{Sr} 1-\mathrm{O} 2^{\mathrm{iii}}$ & $116.73(5)$ & $\mathrm{Sr} 1^{\mathrm{iv}}-\mathrm{Sr} 1-\mathrm{H} 2 \mathrm{~W}$ & $62.1(5)$ \\
\hline $\mathrm{O} 1 \mathrm{~W}-\mathrm{Sr} 1-\mathrm{O} 2^{\mathrm{iii}}$ & $84.18(6)$ & $\mathrm{C} 1-\mathrm{O} 1-\mathrm{H} 1 \mathrm{O}$ & $109(3)$ \\
\hline $\mathrm{O} 1 \mathrm{~W}^{\mathrm{i}}-\mathrm{Sr} 1-\mathrm{O} 2^{\mathrm{iii}}$ & $152.75(6)$ & $\mathrm{C} 7-\mathrm{O} 2-\mathrm{Sr} 1^{\mathrm{ii}}$ & $90.36(14)$ \\
\hline $\mathrm{O} 3^{\mathrm{ii}}-\mathrm{Sr} 1-\mathrm{O} 2^{\mathrm{iii}}$ & $72.53(5)$ & $\mathrm{C} 7-\mathrm{O} 3-\mathrm{Sr} 1$ & $150.19(16)$ \\
\hline 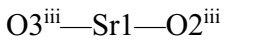 & $48.61(5)$ & $\mathrm{C} 7-\mathrm{O} 3-\mathrm{Sr}^{\mathrm{ii}}$ & $97.06(15)$ \\
\hline $\mathrm{O} 2^{\mathrm{ii}}-\mathrm{Sr} 1-\mathrm{O} 2^{\mathrm{iii}}$ & $97.18(7)$ & $\mathrm{Sr} 1-\mathrm{O} 3-\mathrm{Sr}^{\mathrm{ii}}$ & $107.54(6)$ \\
\hline $\mathrm{O} 3-\mathrm{Sr} 1-\mathrm{C}^{\mathrm{ii}}$ & $95.74(6)$ & $\mathrm{Sr} 1-\mathrm{O} 1 \mathrm{~W}-\mathrm{H} 1 \mathrm{~W}$ & $128(3)$ \\
\hline $\mathrm{O} 3^{\mathrm{i}}-\mathrm{Sr} 1-\mathrm{C}^{\mathrm{ii}}$ & $107.11(6)$ & $\mathrm{Sr} 1-\mathrm{O} 1 \mathrm{~W}-\mathrm{H} 2 \mathrm{~W}$ & $104(2)$ \\
\hline $\mathrm{O} 1 \mathrm{~W}-\mathrm{Sr} 1-\mathrm{C}^{\mathrm{ii}}$ & $163.67(7)$ & $\mathrm{H} 1 \mathrm{~W}-\mathrm{O} 1 \mathrm{~W}-\mathrm{H} 2 \mathrm{~W}$ & $116(4)$ \\
\hline $\mathrm{O} 1 \mathrm{~W}^{\mathrm{i}}-\mathrm{Sr} 1-\mathrm{C}^{\mathrm{ii}}$ & $89.37(6)$ & $\mathrm{O} 1-\mathrm{C} 1-\mathrm{C} 2$ & $117.7(2)$ \\
\hline $\mathrm{O} 3^{\mathrm{ii}}-\mathrm{Sr} 1-\mathrm{C}^{\mathrm{ii}}$ & $24.27(6)$ & $\mathrm{O} 1-\mathrm{C} 1-\mathrm{C} 6$ & $121.9(2)$ \\
\hline $\mathrm{O} 3^{\mathrm{iii}}-\mathrm{Sr} 1-\mathrm{C}^{\mathrm{ii}}$ & $75.74(6)$ & $\mathrm{C} 2-\mathrm{C} 1-\mathrm{C} 6$ & $120.4(2)$ \\
\hline $\mathrm{O} 2^{\mathrm{ii}}-\mathrm{Sr} 1-\mathrm{C}^{\mathrm{ii}}$ & $24.74(6)$ & $\mathrm{C} 3-\mathrm{C} 2-\mathrm{C} 1$ & $119.4(3)$ \\
\hline $\mathrm{O} 2^{\mathrm{iii}}-\mathrm{Sr} 1-\mathrm{C}^{\mathrm{ii}}$ & $81.62(6)$ & $\mathrm{C} 3-\mathrm{C} 2-\mathrm{H} 2$ & 120.3 \\
\hline $\mathrm{O} 3-\mathrm{Sr} 1-\mathrm{C} 7^{\mathrm{iii}}$ & $107.11(6)$ & $\mathrm{C} 1-\mathrm{C} 2-\mathrm{H} 2$ & 120.3 \\
\hline $\mathrm{O} 3^{\mathrm{i}}-\mathrm{Sr} 1-\mathrm{C} 7^{\mathrm{iii}}$ & $95.74(6)$ & $\mathrm{C} 2-\mathrm{C} 3-\mathrm{C} 4$ & $121.0(3)$ \\
\hline $\mathrm{O} 1 \mathrm{~W}-\mathrm{Sr} 1-\mathrm{C}^{\mathrm{iii}}$ & $89.37(6)$ & $\mathrm{C} 2-\mathrm{C} 3-\mathrm{H} 3$ & 119.5 \\
\hline $\mathrm{O} 1 \mathrm{~W}^{\mathrm{i}}-\mathrm{Sr} 1-\mathrm{C} 7^{\mathrm{iii}}$ & $163.67(7)$ & $\mathrm{C} 4-\mathrm{C} 3-\mathrm{H} 3$ & 119.5 \\
\hline $\mathrm{O} 3^{\mathrm{ii}}-\mathrm{Sr} 1-\mathrm{C} 7^{\mathrm{iii}}$ & $75.74(6)$ & $\mathrm{C} 5-\mathrm{C} 4-\mathrm{C} 3$ & $119.4(3)$ \\
\hline $\mathrm{O} 3^{\mathrm{iii}}-\mathrm{Sr} 1-\mathrm{C}^{\mathrm{iii}}$ & $24.27(6)$ & $\mathrm{C} 5-\mathrm{C} 4-\mathrm{H} 4$ & 120.3 \\
\hline $\mathrm{O} 2^{\mathrm{ii}}-\mathrm{Sr} 1-\mathrm{C} 7^{\mathrm{iii}}$ & $81.62(6)$ & $\mathrm{C} 3-\mathrm{C} 4-\mathrm{H} 4$ & 120.3 \\
\hline $\mathrm{O} 2^{\mathrm{iii}}-\mathrm{Sr} 1-\mathrm{C} 7^{\mathrm{iii}}$ & $24.74(6)$ & $\mathrm{C} 4-\mathrm{C} 5-\mathrm{C} 6$ & $120.7(3)$ \\
\hline $\mathrm{C}^{\mathrm{ii}}-\mathrm{Sr} 1-\mathrm{C}^{\mathrm{iii}}$ & $74.37(9)$ & $\mathrm{C} 4-\mathrm{C} 5-\mathrm{H} 5$ & 119.6 \\
\hline $\mathrm{O} 3-\mathrm{Sr} 1-\mathrm{Sr} 1^{\mathrm{ii}}$ & $37.05(4)$ & $\mathrm{C} 6-\mathrm{C} 5-\mathrm{H} 5$ & 119.6 \\
\hline $\mathrm{O} 3^{\mathrm{i}}-\mathrm{Sr} 1-\mathrm{Sr} 1^{\mathrm{ii}}$ & $161.98(4)$ & $\mathrm{C} 5-\mathrm{C} 6-\mathrm{C} 1$ & $119.1(2)$ \\
\hline $\mathrm{O} 1 \mathrm{~W}-\mathrm{Sr} 1-\mathrm{Sr} 1^{\mathrm{ii}}$ & $124.40(4)$ & $\mathrm{C} 5-\mathrm{C} 6-\mathrm{C} 7$ & $120.4(2)$ \\
\hline $\mathrm{O} 1 \mathrm{~W}^{\mathrm{i}}-\mathrm{Sr} 1-\mathrm{Sr}^{\mathrm{ii}}$ & $78.63(5)$ & $\mathrm{C} 1-\mathrm{C} 6-\mathrm{C} 7$ & $120.5(2)$ \\
\hline $\mathrm{O} 3^{\mathrm{ii}}-\mathrm{Sr} 1-\mathrm{Sr} 1^{\mathrm{ii}}$ & $35.40(4)$ & $\mathrm{O} 3-\mathrm{C} 7-\mathrm{O} 2$ & $122.0(2)$ \\
\hline $\mathrm{O} 3^{\mathrm{iii}}-\mathrm{Sr} 1-\mathrm{Sr} 1^{\mathrm{ii}}$ & $111.47(4)$ & $\mathrm{O} 3-\mathrm{C} 7-\mathrm{C} 6$ & $119.1(2)$ \\
\hline $\mathrm{O} 2^{\mathrm{ii}}-\mathrm{Sr} 1-\mathrm{Sr} 1^{\mathrm{ii}}$ & $81.70(4)$ & $\mathrm{O} 2-\mathrm{C} 7-\mathrm{C} 6$ & $118.9(2)$ \\
\hline 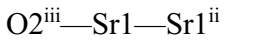 & $74.68(4)$ & $\mathrm{O} 3-\mathrm{C} 7-\mathrm{Sr} 1^{\mathrm{ii}}$ & $58.66(13)$ \\
\hline $\mathrm{C}^{\mathrm{ii}}-\mathrm{Sr} 1-\mathrm{Sr} 1^{\mathrm{ii}}$ & $58.98(5)$ & $\mathrm{O} 2-\mathrm{C} 7-\mathrm{Sr}^{\mathrm{ii}}$ & $64.90(13)$ \\
\hline $\mathrm{C} 7^{\mathrm{iii}}-\mathrm{Sr} 1-\mathrm{Sr} 1^{\mathrm{ii}}$ & $91.32(5)$ & $\mathrm{C} 6-\mathrm{C} 7-\mathrm{Sr} 1^{\mathrm{ii}}$ & $166.48(17)$ \\
\hline $\mathrm{O} 1-\mathrm{C} 1-\mathrm{C} 2-\mathrm{C} 3$ & $-177.6(2)$ & 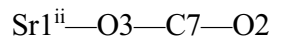 & $15.1(3)$ \\
\hline $\mathrm{C} 6-\mathrm{C} 1-\mathrm{C} 2-\mathrm{C} 3$ & $2.8(4)$ & $\mathrm{Sr} 1-\mathrm{O} 3-\mathrm{C} 7-\mathrm{C} 6$ & $49.6(4)$ \\
\hline $\mathrm{C} 1-\mathrm{C} 2-\mathrm{C} 3-\mathrm{C} 4$ & $-1.1(4)$ & $\mathrm{Sr} 1^{\mathrm{ii}}-\mathrm{O} 3-\mathrm{C} 7-\mathrm{C} 6$ & $-164.54(19)$ \\
\hline $\mathrm{C} 2-\mathrm{C} 3-\mathrm{C} 4-\mathrm{C} 5$ & $-1.1(4)$ & $\mathrm{Sr} 1-\mathrm{O} 3-\mathrm{C} 7-\mathrm{Sr} 1^{\mathrm{ii}}$ & $-145.8(3)$ \\
\hline
\end{tabular}




$\begin{array}{llll}\mathrm{C} 3-\mathrm{C} 4-\mathrm{C} 5-\mathrm{C} 6 & 1.7(4) & \mathrm{Sr} 1{ }^{\mathrm{ii}}-\mathrm{O} 2-\mathrm{C} 7-\mathrm{O} 3 & -14.2(2) \\ \mathrm{C} 4-\mathrm{C} 5-\mathrm{C} 6-\mathrm{C} 1 & -0.1(4) & \mathrm{Sr} 1{ }^{\mathrm{ii}}-\mathrm{O} 2-\mathrm{C} 7-\mathrm{C} 6 & 165.4(2) \\ \mathrm{C} 4-\mathrm{C} 5-\mathrm{C} 6-\mathrm{C} 7 & -179.5(3) & \mathrm{C} 5-\mathrm{C} 6-\mathrm{C} 7-\mathrm{O} 3 & 19.3(4) \\ \mathrm{O} 1-\mathrm{C} 1-\mathrm{C} 6-\mathrm{C} 5 & 178.2(2) & \mathrm{C} 1-\mathrm{C} 6-\mathrm{C} 7-\mathrm{O} 3 & -160.1(2) \\ \mathrm{C} 2-\mathrm{C} 1-\mathrm{C} 6-\mathrm{C} 5 & -2.2(4) & \mathrm{C} 5-\mathrm{C} 6-\mathrm{C} 7-\mathrm{O} 2 & 20.2(4) \\ \mathrm{O} 1-\mathrm{C} 1-\mathrm{C} 6-\mathrm{C} 7 & -2.3(4) & \mathrm{C} 1-\mathrm{C} 6-\mathrm{C} 7-\mathrm{O} 2 & -57.5(8) \\ \mathrm{C} 2-\mathrm{C} 1-\mathrm{C} 6-\mathrm{C} 7 & 177.3(2) & \mathrm{C} 5-\mathrm{C} 6-\mathrm{C} 7-\mathrm{Sr} 1^{\mathrm{ii}} & 123.1(7) \\ \mathrm{Sr} 1-\mathrm{O} 3-\mathrm{C} 7-\mathrm{O} 2 & -130.7(3) & \mathrm{C} 1-\mathrm{C} 6-\mathrm{C} 7-\mathrm{Sr} 1^{\mathrm{ii}} & \end{array}$

Symmetry codes: (i) $-x+1, y,-z-1 / 2$; (ii) $-x+1,-y+1,-z$; (iii) $x,-y+1, z-1 / 2$; (iv) $-x+1,-y+1,-z-1$.

Hydrogen-bond geometry $\left(A,{ }^{\circ}\right)$

\begin{tabular}{lllll}
\hline$D-\mathrm{H} \cdots A$ & $D-\mathrm{H}$ & $\mathrm{H} \cdots A$ & $D \cdots A$ & $D-\mathrm{H} \cdots A$ \\
\hline $\mathrm{O} 1-\mathrm{H} 1 O \cdots \mathrm{O} 2$ & $0.83(2)$ & $1.89(3)$ & $2.604(2)$ & $145(4)$ \\
$\mathrm{O} 1 W-\mathrm{H} 1 W \cdots \mathrm{O} 1^{\mathrm{v}}$ & $0.82(2)$ & $2.01(2)$ & $2.823(3)$ & $172(4)$ \\
$\mathrm{O} 1 W-\mathrm{H} 2 W \cdots \mathrm{O} 2^{\mathrm{vi}}$ & $0.84(2)$ & $2.08(2)$ & $2.925(3)$ & $178(4)$
\end{tabular}

Symmetry codes: (v) $-x+1 / 2,-y+1 / 2,-z$; (vi) $x, y, z-1$.

\section{(SrBa)}

\section{Crystal data}

$\mathrm{C}_{14} \mathrm{H}_{14} \mathrm{Ba}_{0.27} \mathrm{O}_{8} \mathrm{Sr}_{0.73}$

$M_{r}=411.35$

Monoclinic, $C 2 / c$

$a=16.8381(7) \AA$

$b=11.4349(5) \AA$

$c=7.9433(3) \AA$

$\beta=91.280(4)^{\circ}$

$V=1529.04(11) \AA^{3}$

$Z=4$

\section{Data collection}

Oxford Diffraction Xcalibur E diffractometer

Radiation source: sealed tube $\omega$ scans
$F(000)=820$

$D_{\mathrm{x}}=1.787 \mathrm{Mg} \mathrm{m}^{-3}$

Mo $K \alpha$ radiation, $\lambda=0.71073 \AA$

Cell parameters from 7047 reflections

$\theta=3.3-29.8^{\circ}$

$\mu=3.31 \mathrm{~mm}^{-1}$

$T=150 \mathrm{~K}$

Cut prism, colourless

$0.28 \times 0.25 \times 0.15 \mathrm{~mm}$

\section{Refinement}

Refinement on $F^{2}$

Least-squares matrix: full

$R\left[F^{2}>2 \sigma\left(F^{2}\right)\right]=0.021$

$w R\left(F^{2}\right)=0.044$

$S=1.17$

1754 reflections

118 parameters

0 restraints

Hydrogen site location: mixed 
$\mathrm{H}$ atoms treated by a mixture of independent and constrained refinement

$w=1 /\left[\sigma^{2}\left(F_{\mathrm{o}}^{2}\right)+(0.0132 P)^{2}+1.6161 P\right]$

where $P=\left(F_{\mathrm{o}}{ }^{2}+2 F_{\mathrm{c}}{ }^{2}\right) / 3$

$$
\begin{aligned}
& (\Delta / \sigma)_{\max }=0.001 \\
& \Delta \rho_{\max }=0.35 \text { e } \AA^{-3} \\
& \Delta \rho_{\min }=-0.31 \text { e } \AA^{-3}
\end{aligned}
$$

\section{Special details}

Geometry. All e.s.d.'s (except the e.s.d. in the dihedral angle between two l.s. planes) are estimated using the full covariance matrix. The cell e.s.d.'s are taken into account individually in the estimation of e.s.d.'s in distances, angles and torsion angles; correlations between e.s.d.'s in cell parameters are only used when they are defined by crystal symmetry. An approximate (isotropic) treatment of cell e.s.d.'s is

\begin{tabular}{|c|c|c|c|c|c|}
\hline & $x$ & $y$ & $z$ & $U_{\text {iso }} * / U_{\text {eq }}$ & Occ. $(<1)$ \\
\hline Sr1 & 0.5000 & $0.43902(2)$ & -0.2500 & $0.01477(7)$ & $0.729(4)$ \\
\hline Ba1 & 0.5000 & $0.43902(2)$ & -0.2500 & $0.01477(7)$ & $0.271(4)$ \\
\hline $\mathrm{O} 1$ & $0.25640(8)$ & $0.25403(12)$ & $0.26510(18)$ & $0.0233(3)$ & \\
\hline $\mathrm{O} 2$ & $0.37639(8)$ & $0.39626(11)$ & $0.26415(17)$ & $0.0215(3)$ & \\
\hline $\mathrm{O} 1 \mathrm{~W}$ & $0.39280(9)$ & $0.31015(15)$ & $-0.3903(2)$ & $0.0335(4)$ & \\
\hline $\mathrm{O} 3$ & $0.45452(8)$ & $0.38793(11)$ & $0.04491(18)$ & $0.0233(3)$ & \\
\hline $\mathrm{C} 1$ & $0.29899(10)$ & $0.18637(16)$ & $0.1589(2)$ & $0.0175(4)$ & \\
\hline $\mathrm{C} 2$ & $0.26877(12)$ & $0.07775(16)$ & $0.1138(2)$ & $0.0226(4)$ & \\
\hline $\mathrm{H} 2$ & 0.2188 & 0.0530 & 0.1541 & $0.027 *$ & \\
\hline $\mathrm{C} 3$ & $0.31155(13)$ & 0.00609 (18) & $0.0105(3)$ & $0.0267(5)$ & \\
\hline H3 & 0.2908 & -0.0685 & -0.0195 & $0.032 *$ & \\
\hline $\mathrm{C} 4$ & $0.38438(13)$ & $0.04069(17)$ & -0.0508 & $0.0276(5)$ & \\
\hline $\mathrm{H} 4$ & 0.4139 & -0.0103 & -0.1201 & $0.033^{*}$ & \\
\hline C5 & $0.41326(11)$ & 0.14997 (17) & $-0.0100(2)$ & $0.0223(4)$ & \\
\hline H5 & 0.4625 & 0.1748 & -0.0539 & $0.027 *$ & \\
\hline C6 & $0.37156(11)$ & $0.22479(15)$ & $0.0947(2)$ & $0.0172(4)$ & \\
\hline $\mathrm{C} 7$ & $0.40255(10)$ & $0.34315(16)$ & $0.1364(2)$ & $0.0176(4)$ & \\
\hline $\mathrm{H} 1 \mathrm{~W}$ & $0.3492(17)$ & $0.287(2)$ & $-0.361(3)$ & $0.048(8)^{*}$ & \\
\hline $\mathrm{H} 2 \mathrm{~W}$ & $0.3864(16)$ & $0.326(2)$ & $-0.495(4)$ & $0.048(8)^{*}$ & \\
\hline $\mathrm{H} 7$ & $0.2843(16)$ & $0.319(2)$ & $0.285(3)$ & $0.052(8)^{*}$ & \\
\hline
\end{tabular}
used for estimating e.s.d.'s involving l.s. planes.

Fractional atomic coordinates and isotropic or equivalent isotropic displacement parameters $\left(\AA^{2}\right)$

Atomic displacement parameters $\left(\AA^{2}\right)$

\begin{tabular}{lllllll}
\hline & $U^{11}$ & $U^{22}$ & $U^{33}$ & $U^{12}$ & $U^{13}$ & $U^{23}$ \\
\hline Sr1 & $0.01305(10)$ & $0.01757(11)$ & $0.01383(11)$ & 0.000 & $0.00353(7)$ & 0.000 \\
Ba1 & $0.01305(10)$ & $0.01757(11)$ & $0.01383(11)$ & 0.000 & $0.00353(7)$ & 0.000 \\
O1 & $0.0196(7)$ & $0.0219(7)$ & $0.0288(8)$ & $-0.0043(6)$ & $0.0080(6)$ & $-0.0023(6)$ \\
O2 & $0.0198(7)$ & $0.0189(6)$ & $0.0261(8)$ & $-0.0030(5)$ & $0.0063(6)$ & $-0.0051(6)$ \\
O1W & $0.0254(8)$ & $0.0447(10)$ & $0.0307(9)$ & $-0.0075(7)$ & $0.0081(7)$ & $0.0061(8)$ \\
O3 & $0.0209(7)$ & $0.0200(7)$ & $0.0293(8)$ & $-0.0053(5)$ & $0.0095(6)$ & $-0.0022(6)$ \\
C1 & $0.0179(9)$ & $0.0187(9)$ & $0.0159(9)$ & $-0.0006(7)$ & $0.0001(7)$ & $0.0037(7)$ \\
C2 & $0.0255(10)$ & $0.0219(10)$ & $0.0204(10)$ & $-0.0077(8)$ & $-0.0009(8)$ & $0.0037(8)$ \\
C3 & $0.0384(12)$ & $0.0184(10)$ & $0.0231(11)$ & $-0.0072(9)$ & $-0.0016(9)$ & $0.0001(8)$ \\
C4 & $0.0357(11)$ & $0.0196(10)$ & $0.0276(11)$ & $0.0012(8)$ & $0.0044(9)$ & $-0.0041(8)$ \\
C5 & $0.0227(9)$ & $0.0207(9)$ & $0.0238(10)$ & $-0.0001(8)$ & $0.0055(8)$ & $0.0002(8)$ \\
C6 & $0.0173(8)$ & $0.0161(9)$ & $0.0183(9)$ & $-0.0018(7)$ & $-0.0006(7)$ & $0.0007(7)$ \\
C7 & $0.0132(8)$ & $0.0177(9)$ & $0.0219(10)$ & $0.0003(7)$ & $0.0001(7)$ & $0.0009(7)$ \\
\end{tabular}


Geometric parameters $\left(\AA,{ }^{\circ}\right)$

\begin{tabular}{|c|c|c|c|}
\hline $\mathrm{Sr} 1-\mathrm{O} 3^{\mathrm{i}}$ & $2.5486(13)$ & $\mathrm{O} 1 \mathrm{~W}-\mathrm{H} 2 \mathrm{~W}$ & $0.86(3)$ \\
\hline $\mathrm{Sr} 1-\mathrm{O} 3$ & $2.5486(13)$ & $\mathrm{O} 3-\mathrm{C} 7$ & $1.259(2)$ \\
\hline $\mathrm{Sr} 1-\mathrm{O} 1 \mathrm{~W}^{\mathrm{i}}$ & $2.5654(17)$ & $\mathrm{O} 3-\mathrm{Ba}^{\mathrm{ii}}$ & $2.6646(13)$ \\
\hline $\mathrm{Sr} 1-\mathrm{O} 1 \mathrm{~W}$ & $2.5655(17)$ & $\mathrm{O} 3-\mathrm{Sr} 1^{\mathrm{ii}}$ & $2.6646(13)$ \\
\hline $\mathrm{Sr} 1-\mathrm{O} 3^{\mathrm{ii}}$ & $2.6647(13)$ & $\mathrm{C} 1-\mathrm{C} 2$ & $1.386(3)$ \\
\hline $\mathrm{Sr} 1-\mathrm{O} 3^{\mathrm{iii}}$ & $2.6647(13)$ & $\mathrm{C} 1-\mathrm{C} 6$ & $1.405(2)$ \\
\hline $\mathrm{Sr} 1-\mathrm{O} 2^{\mathrm{ii}}$ & $2.8113(13)$ & $\mathrm{C} 2-\mathrm{C} 3$ & $1.375(3)$ \\
\hline $\mathrm{Sr} 1-\mathrm{O} 2^{\mathrm{iii}}$ & $2.8113(13)$ & $\mathrm{C} 2-\mathrm{H} 2$ & 0.9500 \\
\hline $\mathrm{Sr} 1-\mathrm{C} 7^{\mathrm{ii}}$ & $3.1056(18)$ & $\mathrm{C} 3-\mathrm{C} 4$ & $1.387(3)$ \\
\hline $\mathrm{Sr} 1-\mathrm{C}^{\mathrm{iii}}$ & $3.1056(18)$ & $\mathrm{C} 3-\mathrm{H} 3$ & 0.9500 \\
\hline $\mathrm{Sr} 1-\mathrm{Ba} 1^{\mathrm{ii}}$ & $4.2094(2)$ & $\mathrm{C} 4-\mathrm{C} 5$ & $1.377(3)$ \\
\hline $\mathrm{Sr} 1-\mathrm{Sr} 1^{\mathrm{ii}}$ & $4.2094(2)$ & $\mathrm{C} 4-\mathrm{H} 4$ & 0.9500 \\
\hline $\mathrm{O} 1-\mathrm{C} 1$ & $1.361(2)$ & $\mathrm{C} 5-\mathrm{C} 6$ & $1.394(3)$ \\
\hline $\mathrm{O} 1-\mathrm{H} 7$ & $0.89(3)$ & $\mathrm{C} 5-\mathrm{H} 5$ & 0.9500 \\
\hline $\mathrm{O} 2-\mathrm{C} 7$ & $1.270(2)$ & $\mathrm{C} 6-\mathrm{C} 7$ & $1.485(2)$ \\
\hline $\mathrm{O} 2-\mathrm{Ba}^{\mathrm{ii}}$ & $2.8112(13)$ & $\mathrm{C} 7-\mathrm{Ba} 1^{\mathrm{ii}}$ & $3.1056(18)$ \\
\hline $\mathrm{O} 2-\mathrm{Sr} 1^{\mathrm{ii}}$ & $2.8112(13)$ & $\mathrm{C} 7-\mathrm{Sr}^{\mathrm{ii}}$ & $3.1056(18)$ \\
\hline $\mathrm{O} 1 \mathrm{~W}-\mathrm{H} 1 \mathrm{~W}$ & $0.82(3)$ & & \\
\hline $\mathrm{O} 3{ }^{\mathrm{i}}-\mathrm{Sr} 1-\mathrm{O} 3$ & $153.49(6)$ & $\mathrm{C} 7^{\mathrm{iii}}-\mathrm{Sr} 1-\mathrm{Ba} 1^{\mathrm{ii}}$ & $89.85(4)$ \\
\hline $\mathrm{O} 3^{\mathrm{i}}-\mathrm{Sr} 1-\mathrm{O} 1 \mathrm{~W}^{\mathrm{i}}$ & $92.55(5)$ & $\mathrm{O} 3^{\mathrm{i}}-\mathrm{Sr} 1-\mathrm{Sr} 1^{\mathrm{ii}}$ & $161.72(3)$ \\
\hline $\mathrm{O} 3-\mathrm{Sr} 1-\mathrm{O} 1 \mathrm{~W}^{\mathrm{i}}$ & $72.06(5)$ & $\mathrm{O} 3-\mathrm{Sr} 1-\mathrm{Sr} 1^{\mathrm{ii}}$ & $37.10(3)$ \\
\hline $\mathrm{O} 3{ }^{\mathrm{i}}-\mathrm{Sr} 1-\mathrm{O} 1 \mathrm{~W}$ & $72.06(5)$ & $\mathrm{O} 1 \mathrm{~W}^{\mathrm{i}}-\mathrm{Sr} 1-\mathrm{Sr} 1^{\mathrm{ii}}$ & $78.19(4)$ \\
\hline $\mathrm{O} 3-\mathrm{Sr} 1-\mathrm{O} 1 \mathrm{~W}$ & $92.56(5)$ & $\mathrm{O} 1 \mathrm{~W}-\mathrm{Sr} 1-\mathrm{Sr} 1^{\mathrm{ii}}$ & $125.82(4)$ \\
\hline $\mathrm{O} 1 \mathrm{~W}^{\mathrm{i}}-\mathrm{Sr} 1-\mathrm{O} 1 \mathrm{~W}$ & $109.88(8)$ & $\mathrm{O} 3^{\mathrm{ii}}-\mathrm{Sr} 1-\mathrm{Sr}^{\mathrm{ii}}$ & $35.23(3)$ \\
\hline $\mathrm{O} 3^{\mathrm{i}}-\mathrm{Sr} 1-\mathrm{O} 3^{\mathrm{ii}}$ & $130.09(4)$ & $\mathrm{O} 3^{\mathrm{iii}}-\mathrm{Sr} 1-\mathrm{Sr} 1^{\mathrm{ii}}$ & $108.95(3)$ \\
\hline $\mathrm{O} 3-\mathrm{Sr} 1-\mathrm{O}^{\mathrm{ii}}$ & $72.33(4)$ & $\mathrm{O} 2^{\mathrm{ii}}-\mathrm{Sr} 1-\mathrm{Sr} 1^{\mathrm{ii}}$ & $80.29(3)$ \\
\hline $\mathrm{O} 1 \mathrm{~W}^{\mathrm{i}}-\mathrm{Sr} 1-\mathrm{O} 3^{\mathrm{ii}}$ & $88.35(5)$ & $\mathrm{O} 2^{\mathrm{iii}}-\mathrm{Sr} 1-\mathrm{Sr} 1^{\mathrm{ii}}$ & $74.02(3)$ \\
\hline $\mathrm{O} 1 \mathrm{~W}-\mathrm{Sr} 1-\mathrm{O} 3^{\mathrm{ii}}$ & $151.87(5)$ & $\mathrm{C} 7^{\mathrm{ii}}-\mathrm{Sr} 1-\mathrm{Sr} 1^{\mathrm{ii}}$ & $58.08(3)$ \\
\hline 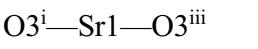 & $72.33(4)$ & $\mathrm{C} 7^{\mathrm{iii}}-\mathrm{Sr} 1-\mathrm{Sr} 1^{\mathrm{ii}}$ & $89.85(4)$ \\
\hline $\mathrm{O} 3-\mathrm{Sr} 1-\mathrm{O} 3^{\mathrm{iii}}$ & $130.09(4)$ & $\mathrm{Ba} 1^{\mathrm{ii}}-\mathrm{Sr} 1-\mathrm{Sr} 1^{\mathrm{ii}}$ & 0.0 \\
\hline $\mathrm{O} 1 \mathrm{~W}^{\mathrm{i}}-\mathrm{Sr} 1-\mathrm{O} 3^{\mathrm{iii}}$ & $151.87(5)$ & $\mathrm{C} 1-\mathrm{O} 1-\mathrm{H} 7$ & $107.6(18)$ \\
\hline $\mathrm{O} 1 \mathrm{~W}-\mathrm{Sr} 1-\mathrm{O}^{\mathrm{iii}}$ & $88.35(5)$ & $\mathrm{C} 7-\mathrm{O} 2-\mathrm{Ba} 1^{\mathrm{ii}}$ & $91.03(10)$ \\
\hline $\mathrm{O} 3^{\mathrm{ii}}-\mathrm{Sr} 1-\mathrm{O} 3^{\mathrm{iii}}$ & $84.09(6)$ & $\mathrm{C} 7-\mathrm{O} 2-\mathrm{Sr} 1^{\mathrm{ii}}$ & $91.03(10)$ \\
\hline $\mathrm{O} 3^{\mathrm{i}}-\mathrm{Sr} 1-\mathrm{O} 2^{\mathrm{ii}}$ & $83.05(4)$ & $\mathrm{Ba} 1^{\mathrm{ii}}-\mathrm{O} 2-\mathrm{Sr} 1^{\mathrm{ii}}$ & 0.0 \\
\hline $\mathrm{O} 3-\mathrm{Sr} 1-\mathrm{O} 2^{\mathrm{ii}}$ & $115.36(4)$ & $\mathrm{Sr} 1-\mathrm{O} 1 \mathrm{~W}-\mathrm{H} 1 \mathrm{~W}$ & $133.7(19)$ \\
\hline $\mathrm{O} 1 \mathrm{~W}^{\mathrm{i}}-\mathrm{Sr} 1-\mathrm{O} 2^{\mathrm{ii}}$ & $83.56(5)$ & $\mathrm{Sr} 1-\mathrm{O} 1 \mathrm{~W}-\mathrm{H} 2 \mathrm{~W}$ & $111.9(18)$ \\
\hline $\mathrm{O} 1 \mathrm{~W}-\mathrm{Sr} 1-\mathrm{O} 2^{\mathrm{ii}}$ & $151.87(5)$ & $\mathrm{H} 1 \mathrm{~W}-\mathrm{O} 1 \mathrm{~W}-\mathrm{H} 2 \mathrm{~W}$ & $104(3)$ \\
\hline $\mathrm{O} 3^{\mathrm{ii}}-\mathrm{Sr} 1-\mathrm{O} 2^{\mathrm{ii}}$ & $47.47(4)$ & $\mathrm{C} 7-\mathrm{O} 3-\mathrm{Sr} 1$ & $148.31(13)$ \\
\hline $\mathrm{O} 3^{\mathrm{iii}}-\mathrm{Sr} 1-\mathrm{O} 2^{\mathrm{ii}}$ & $71.39(4)$ & $\mathrm{C} 7-\mathrm{O} 3-\mathrm{Ba} 1^{\mathrm{ii}}$ & $98.23(11)$ \\
\hline $\mathrm{O} 3^{\mathrm{i}}-\mathrm{Sr} 1-\mathrm{O} 2^{\mathrm{iii}}$ & $115.36(4)$ & $\mathrm{Sr} 1-\mathrm{O} 3-\mathrm{Ba} 1^{\mathrm{ii}}$ & 107.7 \\
\hline $\mathrm{O} 3-\mathrm{Sr} 1-\mathrm{O} 2^{\mathrm{iii}}$ & $83.05(4)$ & $\mathrm{C} 7-\mathrm{O} 3-\mathrm{Sr} 1^{\mathrm{ii}}$ & $98.23(11)$ \\
\hline $\mathrm{O} 1 \mathrm{~W}^{\mathrm{i}}-\mathrm{Sr} 1-\mathrm{O} 2^{\mathrm{iii}}$ & $151.87(5)$ & $\mathrm{Sr} 1-\mathrm{O} 3-\mathrm{Sr} 1^{\mathrm{ii}}$ & $107.67(4)$ \\
\hline $\mathrm{O} 1 \mathrm{~W}-\mathrm{Sr} 1-\mathrm{O} 2^{\mathrm{iii}}$ & $83.56(5)$ & $\mathrm{Ba}^{\mathrm{ii}}-\mathrm{O} 3-\mathrm{Sr} 1^{\mathrm{ii}}$ & 0.0 \\
\hline $\mathrm{O} 3^{\mathrm{ii}}-\mathrm{Sr} 1-\mathrm{O} 2^{\mathrm{iii}}$ & $71.39(4)$ & $\mathrm{O} 1-\mathrm{C} 1-\mathrm{C} 2$ & $118.31(16)$ \\
\hline $\mathrm{O} 3^{\mathrm{iii}}-\mathrm{Sr} 1-\mathrm{O} 2^{\mathrm{iii}}$ & $47.47(4)$ & $\mathrm{O} 1-\mathrm{C} 1-\mathrm{C} 6$ & $121.46(16)$ \\
\hline $\mathrm{O} 2^{\mathrm{ii}}-\mathrm{Sr} 1-\mathrm{O} 2^{\mathrm{iii}}$ & $95.86(5)$ & $\mathrm{C} 2-\mathrm{C} 1-\mathrm{C} 6$ & $120.24(17)$ \\
\hline $\mathrm{O}^{\mathrm{i}}-\mathrm{Sr} 1-\mathrm{C}^{\mathrm{ii}}$ & $106.46(5)$ & $\mathrm{C} 3-\mathrm{C} 2-\mathrm{C} 1$ & $119.61(18)$ \\
\hline $\mathrm{O} 3-\mathrm{Sr} 1-\mathrm{C}^{\mathrm{ii}}$ & $94.84(5)$ & $\mathrm{C} 3-\mathrm{C} 2-\mathrm{H} 2$ & 120.2 \\
\hline $\mathrm{O} 1 \mathrm{~W}^{\mathrm{i}}-\mathrm{Sr} 1-\mathrm{C}^{\mathrm{ii}}$ & $88.42(5)$ & $\mathrm{C} 1-\mathrm{C} 2-\mathrm{H} 2$ & 120.2 \\
\hline $\mathrm{O} 1 \mathrm{~W}-\mathrm{Sr} 1-\mathrm{C} 7^{\mathrm{ii}}$ & $161.62(5)$ & $\mathrm{C} 2-\mathrm{C} 3-\mathrm{C} 4$ & $121.24(18)$ \\
\hline
\end{tabular}




\begin{tabular}{|c|c|c|c|}
\hline $\mathrm{O} 3^{\mathrm{ii}}-\mathrm{Sr} 1-\mathrm{C}^{\mathrm{ii}}$ & $23.65(4)$ & $\mathrm{C} 2-\mathrm{C} 3-\mathrm{H} 3$ & 119.4 \\
\hline $\mathrm{O} 3^{\mathrm{iii}}-\mathrm{Sr} 1-\mathrm{C}^{\mathrm{ii}}$ & $74.03(5)$ & $\mathrm{C} 4-\mathrm{C} 3-\mathrm{H} 3$ & 119.4 \\
\hline $\mathrm{O} 2^{\mathrm{ii}}-\mathrm{Sr} 1-\mathrm{C} 7^{\mathrm{ii}}$ & $24.14(4)$ & $\mathrm{C} 5-\mathrm{C} 4-\mathrm{C} 3$ & $119.10(19)$ \\
\hline $\mathrm{O} 2^{\mathrm{iii}}-\mathrm{Sr} 1-\mathrm{C} 7^{\mathrm{ii}}$ & $80.67(4)$ & $\mathrm{C} 5-\mathrm{C} 4-\mathrm{H} 4$ & 120.4 \\
\hline $\mathrm{O} 3^{\mathrm{i}}-\mathrm{Sr} 1-\mathrm{C} 7^{\mathrm{iii}}$ & $94.84(5)$ & $\mathrm{C} 3-\mathrm{C} 4-\mathrm{H} 4$ & 120.4 \\
\hline $\mathrm{O} 3-\mathrm{Sr} 1-\mathrm{C} 7^{\mathrm{iii}}$ & $106.46(5)$ & $\mathrm{C} 4-\mathrm{C} 5-\mathrm{C} 6$ & $121.18(18)$ \\
\hline $\mathrm{O} 1 \mathrm{~W}^{\mathrm{i}}-\mathrm{Sr} 1-\mathrm{C} 7^{\mathrm{iii}}$ & $161.62(5)$ & $\mathrm{C} 4-\mathrm{C} 5-\mathrm{H} 5$ & 119.4 \\
\hline $\mathrm{O} 1 \mathrm{~W}-\mathrm{Sr} 1-\mathrm{C}^{\mathrm{iii}}$ & $88.42(5)$ & $\mathrm{C} 6-\mathrm{C} 5-\mathrm{H} 5$ & 119.4 \\
\hline $\mathrm{O} 3^{\mathrm{ii}}-\mathrm{Sr} 1-\mathrm{C} 7^{\mathrm{iii}}$ & $74.03(5)$ & $\mathrm{C} 5-\mathrm{C} 6-\mathrm{C} 1$ & $118.58(17)$ \\
\hline $\mathrm{O} 3^{\mathrm{iii}}-\mathrm{Sr} 1-\mathrm{C}^{\mathrm{iii}}$ & $23.65(4)$ & $\mathrm{C} 5-\mathrm{C} 6-\mathrm{C} 7$ & $120.86(16)$ \\
\hline $\mathrm{O} 2^{\mathrm{ii}}-\mathrm{Sr} 1-\mathrm{C} 7^{\mathrm{iii}}$ & $80.67(4)$ & $\mathrm{C} 1-\mathrm{C} 6-\mathrm{C} 7$ & $120.55(16)$ \\
\hline 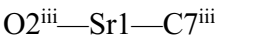 & $24.14(4)$ & $\mathrm{O} 3-\mathrm{C} 7-\mathrm{O} 2$ & $121.66(17)$ \\
\hline $\mathrm{C} 7^{\mathrm{ii}}-\mathrm{Sr} 1-\mathrm{C} 7^{\mathrm{iii}}$ & $73.35(7)$ & $\mathrm{O} 3-\mathrm{C} 7-\mathrm{C} 6$ & $119.18(16)$ \\
\hline $\mathrm{O} 3^{\mathrm{i}}-\mathrm{Sr} 1-\mathrm{Ba} 1^{\mathrm{ii}}$ & $161.72(3)$ & $\mathrm{O} 2-\mathrm{C} 7-\mathrm{C} 6$ & $119.16(16)$ \\
\hline $\mathrm{O} 3-\mathrm{Sr} 1-\mathrm{Ba} 1^{\mathrm{ii}}$ & $37.10(3)$ & $\mathrm{O} 3-\mathrm{C} 7-\mathrm{Ba}^{\mathrm{ii}}$ & $58.12(9)$ \\
\hline $\mathrm{O} 1 \mathrm{~W}^{\mathrm{i}}-\mathrm{Sr} 1-\mathrm{Ba}^{\mathrm{ii}}$ & $78.19(4)$ & $\mathrm{O} 2-\mathrm{C} 7-\mathrm{Ba} 1^{\mathrm{ii}}$ & $64.83(9)$ \\
\hline $\mathrm{O} 1 \mathrm{~W}-\mathrm{Sr} 1-\mathrm{Ba}^{\mathrm{ii}}$ & $125.82(4)$ & $\mathrm{C} 6-\mathrm{C} 7-\mathrm{Ba} 1^{\mathrm{ii}}$ & $167.51(12)$ \\
\hline $\mathrm{O} 3^{\mathrm{ii}}-\mathrm{Sr} 1-\mathrm{Ba}^{\mathrm{ii}}$ & $35.23(3)$ & $\mathrm{O} 3-\mathrm{C} 7-\mathrm{Sr}^{\mathrm{ii}}$ & $58.12(9)$ \\
\hline $\mathrm{O} 3^{\mathrm{iii}}-\mathrm{Sr} 1-\mathrm{Ba} 1^{\mathrm{ii}}$ & $108.95(3)$ & $\mathrm{O} 2-\mathrm{C} 7-\mathrm{Sr}^{\mathrm{ii}}$ & $64.83(9)$ \\
\hline $\mathrm{O} 2^{\mathrm{ii}}-\mathrm{Sr} 1-\mathrm{Ba} 1^{\mathrm{ii}}$ & $80.29(3)$ & $\mathrm{C} 6-\mathrm{C} 7-\mathrm{Sr} 1^{\mathrm{ii}}$ & $167.51(12)$ \\
\hline $\mathrm{O} 2^{\mathrm{iii}}-\mathrm{Sr} 1-\mathrm{Ba} 1^{\mathrm{ii}}$ & $74.02(3)$ & $\mathrm{Ba}^{\mathrm{ii}}-\mathrm{C} 7-\mathrm{Sr} 1^{\mathrm{ii}}$ & 0.0 \\
\hline $\mathrm{C}^{\mathrm{ii}}-\mathrm{Sr} 1-\mathrm{Ba} 1^{\mathrm{ii}}$ & $58.08(3)$ & & \\
\hline $\mathrm{O} 1-\mathrm{C} 1-\mathrm{C} 2-\mathrm{C} 3$ & $-178.19(18)$ & $\mathrm{Sr}^{\mathrm{ii}}-\mathrm{O} 3-\mathrm{C} 7-\mathrm{Ba}^{\mathrm{ii}}$ & 0.0 \\
\hline $\mathrm{C} 6-\mathrm{C} 1-\mathrm{C} 2-\mathrm{C} 3$ & $2.2(3)$ & $\mathrm{Sr} 1-\mathrm{O} 3-\mathrm{C} 7-\mathrm{Sr}{ }^{\mathrm{ii}}$ & $-144.9(2)$ \\
\hline $\mathrm{C} 1-\mathrm{C} 2-\mathrm{C} 3-\mathrm{C} 4$ & $-0.5(3)$ & $\mathrm{Ba} 1^{\mathrm{ii}}-\mathrm{O} 3-\mathrm{C} 7-\mathrm{Sr} 1^{\mathrm{ii}}$ & 0.0 \\
\hline $\mathrm{C} 2-\mathrm{C} 3-\mathrm{C} 4-\mathrm{C} 5$ & $-1.4(3)$ & $\mathrm{Ba} 1 \stackrel{\mathrm{ii}}{-} \mathrm{O} 2-\mathrm{C} 7-\mathrm{O} 3$ & $-12.76(18)$ \\
\hline $\mathrm{C} 3-\mathrm{C} 4-\mathrm{C} 5-\mathrm{C} 6$ & $1.6(3)$ & $\mathrm{Sr} 1^{\mathrm{ii}}-\mathrm{O} 2-\mathrm{C} 7-\mathrm{O} 3$ & $-12.76(18)$ \\
\hline $\mathrm{C} 4-\mathrm{C} 5-\mathrm{C} 6-\mathrm{C} 1$ & $0.2(3)$ & $\mathrm{Ba} 1^{\mathrm{ii}}-\mathrm{O} 2-\mathrm{C} 7-\mathrm{C} 6$ & $166.69(15)$ \\
\hline $\mathrm{C} 4-\mathrm{C} 5-\mathrm{C} 6-\mathrm{C} 7$ & $-179.17(19)$ & $\mathrm{Sr} 1^{\mathrm{ii}}-\mathrm{O} 2-\mathrm{C} 7-\mathrm{C} 6$ & $166.69(15)$ \\
\hline $\mathrm{O} 1-\mathrm{C} 1-\mathrm{C} 6-\mathrm{C} 5$ & $178.34(17)$ & $\mathrm{Sr} 1^{\mathrm{ii}}-\mathrm{O} 2-\mathrm{C} 7-\mathrm{Ba} 1^{\mathrm{ii}}$ & 0.0 \\
\hline $\mathrm{C} 2-\mathrm{C} 1-\mathrm{C} 6-\mathrm{C} 5$ & $-2.1(3)$ & $\mathrm{Ba} 1^{\mathrm{ii}}-\mathrm{O} 2-\mathrm{C} 7-\mathrm{Sr} 1^{\mathrm{ii}}$ & 0.0 \\
\hline $\mathrm{O} 1-\mathrm{C} 1-\mathrm{C} 6-\mathrm{C} 7$ & $-2.3(3)$ & $\mathrm{C} 5-\mathrm{C} 6-\mathrm{C} 7-\mathrm{O} 3$ & $18.5(3)$ \\
\hline $\mathrm{C} 2-\mathrm{C} 1-\mathrm{C} 6-\mathrm{C} 7$ & $177.26(17)$ & $\mathrm{C} 1-\mathrm{C} 6-\mathrm{C} 7-\mathrm{O} 3$ & $-160.87(18)$ \\
\hline $\mathrm{Sr} 1-\mathrm{O} 3-\mathrm{C} 7-\mathrm{O} 2$ & $-131.29(19)$ & $\mathrm{C} 5-\mathrm{C} 6-\mathrm{C} 7-\mathrm{O} 2$ & $-161.00(18)$ \\
\hline $\mathrm{Ba}^{\mathrm{ii}}-\mathrm{O} 3-\mathrm{C} 7-\mathrm{O} 2$ & $13.61(19)$ & $\mathrm{C} 1-\mathrm{C} 6-\mathrm{C} 7-\mathrm{O} 2$ & $19.7(3)$ \\
\hline $\mathrm{Sr}^{\mathrm{ii}}-\mathrm{O} 3-\mathrm{C} 7-\mathrm{O} 2$ & $13.61(19)$ & $\mathrm{C} 5-\mathrm{C} 6-\mathrm{C} 7-\mathrm{Ba}^{\mathrm{ii}}$ & $-55.5(7)$ \\
\hline $\mathrm{Sr} 1-\mathrm{O} 3-\mathrm{C} 7-\mathrm{C} 6$ & $49.3(3)$ & $\mathrm{C} 1-\mathrm{C} 6-\mathrm{C} 7-\mathrm{Ba}^{\mathrm{ii}}$ & $125.1(5)$ \\
\hline $\mathrm{Ba} 1^{\mathrm{ii}}-\mathrm{O} 3-\mathrm{C} 7-\mathrm{C} 6$ & $-165.83(14)$ & $\mathrm{C} 5-\mathrm{C} 6-\mathrm{C} 7-\mathrm{Sr}^{\mathrm{ii}}$ & $-55.5(7)$ \\
\hline $\mathrm{Sr} 1^{\mathrm{ii}}-\mathrm{O} 3-\mathrm{C} 7-\mathrm{C} 6$ & $-165.83(14)$ & $\mathrm{C} 1-\mathrm{C} 6-\mathrm{C} 7-\mathrm{Sr}^{\mathrm{ii}}$ & $125.1(5)$ \\
\hline $\mathrm{Sr} 1-\mathrm{O} 3-\mathrm{C} 7-\mathrm{Ba}^{\mathrm{ii}}$ & $-144.9(2)$ & & \\
\hline
\end{tabular}

Symmetry codes: (i) $-x+1, y,-z-1 / 2$; (ii) $-x+1,-y+1,-z$; (iii) $x,-y+1, z-1 / 2$.

Hydrogen-bond geometry $\left(\AA,{ }^{\circ}\right)$

\begin{tabular}{lllll}
\hline$D-\mathrm{H} \cdots A$ & $D-\mathrm{H}$ & $\mathrm{H} \cdots A$ & $D \cdots A$ & $D-\mathrm{H} \cdots A$ \\
\hline $\mathrm{O} 1-\mathrm{H} 7 \cdots \mathrm{O} 2$ & $0.89(3)$ & $1.80(3)$ & $2.5938(18)$ & $148(3)$ \\
$\mathrm{O} 1 W-\mathrm{H} 1 W \cdots \mathrm{O} 1^{\text {iv }}$ & $0.82(3)$ & $2.00(3)$ & $2.820(2)$ & $172(3)$ \\
$\mathrm{O} 1 W-\mathrm{H} 2 W \cdots \mathrm{O} 2^{\mathrm{v}}$ & $0.86(3)$ & $2.08(3)$ & $2.923(2)$ & $169(3)$ \\
\hline
\end{tabular}

Symmetry codes: (iv) $-x+1 / 2,-y+1 / 2,-z$; (v) $x, y, z-1$. 